\title{
Smart and Green Energy (SAGE) for Base Camps Final Report
}

$M$ Engels

PA Boyd

TM Koehler

$S$ Goel
DR Sisk

D Hatley

V Mendon

$\mathrm{J}$ Hail

January 2014

Pacific Northwest

NATIONAL LABORATORY

Proudly Operated by Battelle Since 1965 


\title{
DISCLAIMER
}

This report was prepared as an account of work sponsored by an agency of the United States Government. Neither the United States Government nor any agency thereof, nor Battelle Memorial Institute, nor any of their employees, makes any warranty, express or implied, or assumes any legal liability or responsibility for the accuracy, completeness, or usefulness of any information, apparatus, product, or process disclosed, or represents that its use would not infringe privately owned rights. Reference herein to any specific commercial product, process, or service by trade name, trademark, manufacturer, or otherwise does not necessarily constitute or imply its endorsement, recommendation, or favoring by the United States Government or any agency thereof, or Battelle Memorial Institute. The views and opinions of authors expressed herein do not necessarily state or reflect those of the United States Government or any agency thereof.

\author{
PACIFIC NORTHWEST NATIONAL LABORATORY \\ operated by \\ BATTELLE \\ for the \\ UNITED STATES DEPARTMENT OF ENERGY \\ under Contract DE-AC05-76RL01830
}

Printed in the United States of America
Available to DOE and DOE contractors from the Office of Scientific and Technical Information,
P.O. Box 62, Oak Ridge, TN 37831-0062;
ph: (865) 576-8401
fax: $(865)$ 576-5728
email: reports@adonis.osti.gov

\begin{abstract}
Available to the public from the National Technical Information Service, U.S. Department of Commerce, 5285 Port Royal Rd., Springfield, VA 22161 ph: (800) 553-6847 fax: $(703) 605-6900$ email: orders@ntis.fedworld.gov online ordering: http://www.ntis.gov/ordering.htm
\end{abstract}

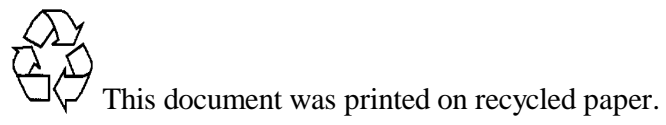




\section{Smart and Green Energy (SAGE) for Base Camps Final Report}

$\begin{array}{ll}\text { M Engels } & \text { DR Sisk } \\ \text { PA Boyd } & \text { D Hatley } \\ \text { TM Koehler } & \text { V Mendon } \\ \text { S Goel } & \text { J Hail }\end{array}$

January 2014

Prepared for

the U.S. Department of Energy

under Contract DE-AC05-76RL01830

Pacific Northwest National Laboratory

Richland, Washington 99354 



\section{Executive Summary}

The U.S. Army Logistics Innovation Agency (LIA), in conjunction with the Department of Energy's Pacific Northwest National Laboratory (PNNL), conducted an evaluation and demonstration of Smart and Green Energy (SAGE) commercial off-the-shelf (COTS) technologies to improve energy efficiency and reduce the quantity of fuel needed to operate base camps. Field tests and calibrated energy models demonstrated that significant fuel reductions of $49 \%$ to $84 \%$ are achievable depending on camp size and location.

The SAGE project integrated the following COTS technologies to reduce fuel use at extra small, small, and medium size base camps (i.e., 150-, 600-, and 5,000-Soldier camps):

- a smart microgrid with a common control system that coupled multiple diesel generators

- an energy storage system

- two renewable energy sources: a solar hot water (SHW) system to supplement fuel-fired water heaters with preheated water, and two solar electric photovoltaic (PV) arrays

- several energy efficient, insulated, modular shelters for troop housing with high-efficiency heat pumps

- a prototype base camp energy management system that actively monitored and managed base camp equipment and buildings.

See Figure ES-1 for a schematic of the SAGE base camp configuration and Figure ES-2 for an aerial view of the SAGE base camp as deployed and tested at the Base Camp Integration Laboratory (BCIL) at Fort Devens, Massachusetts.

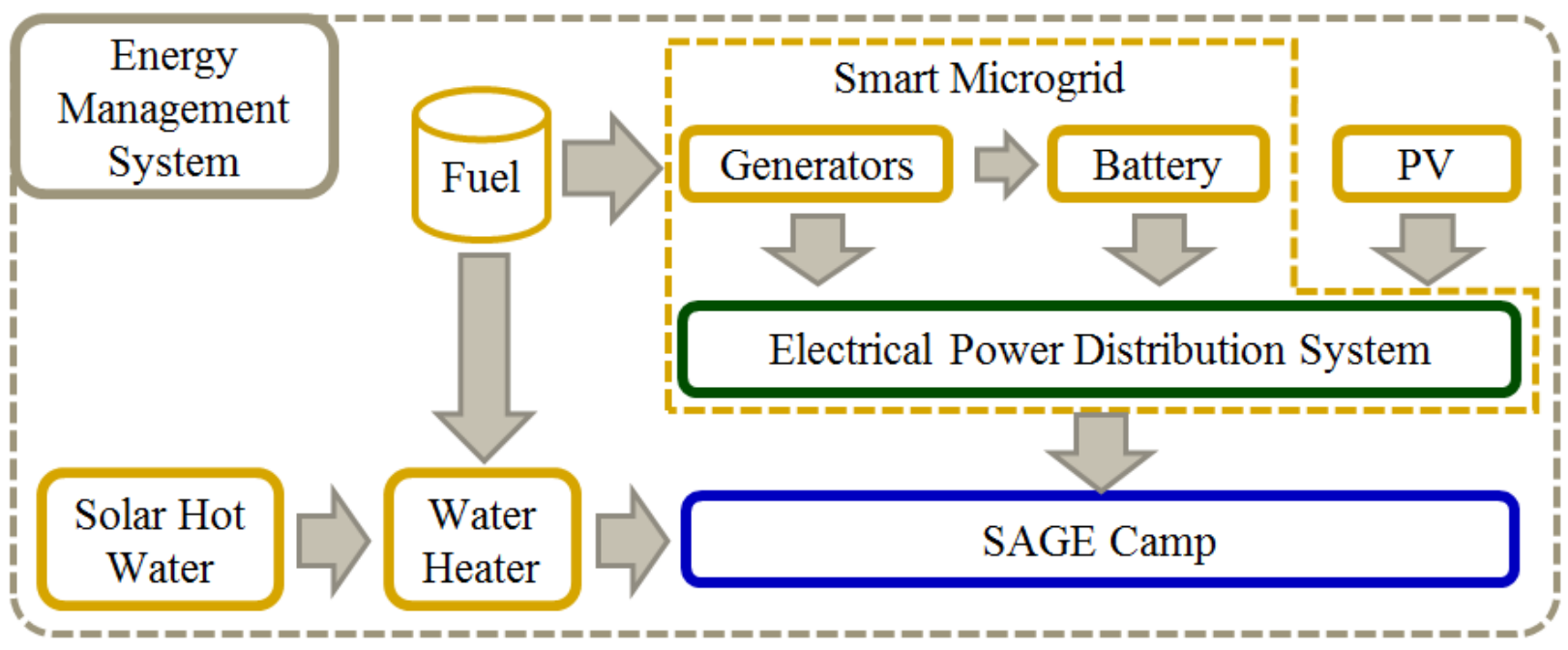

Figure ES-1. SAGE Technology Overview Diagram 


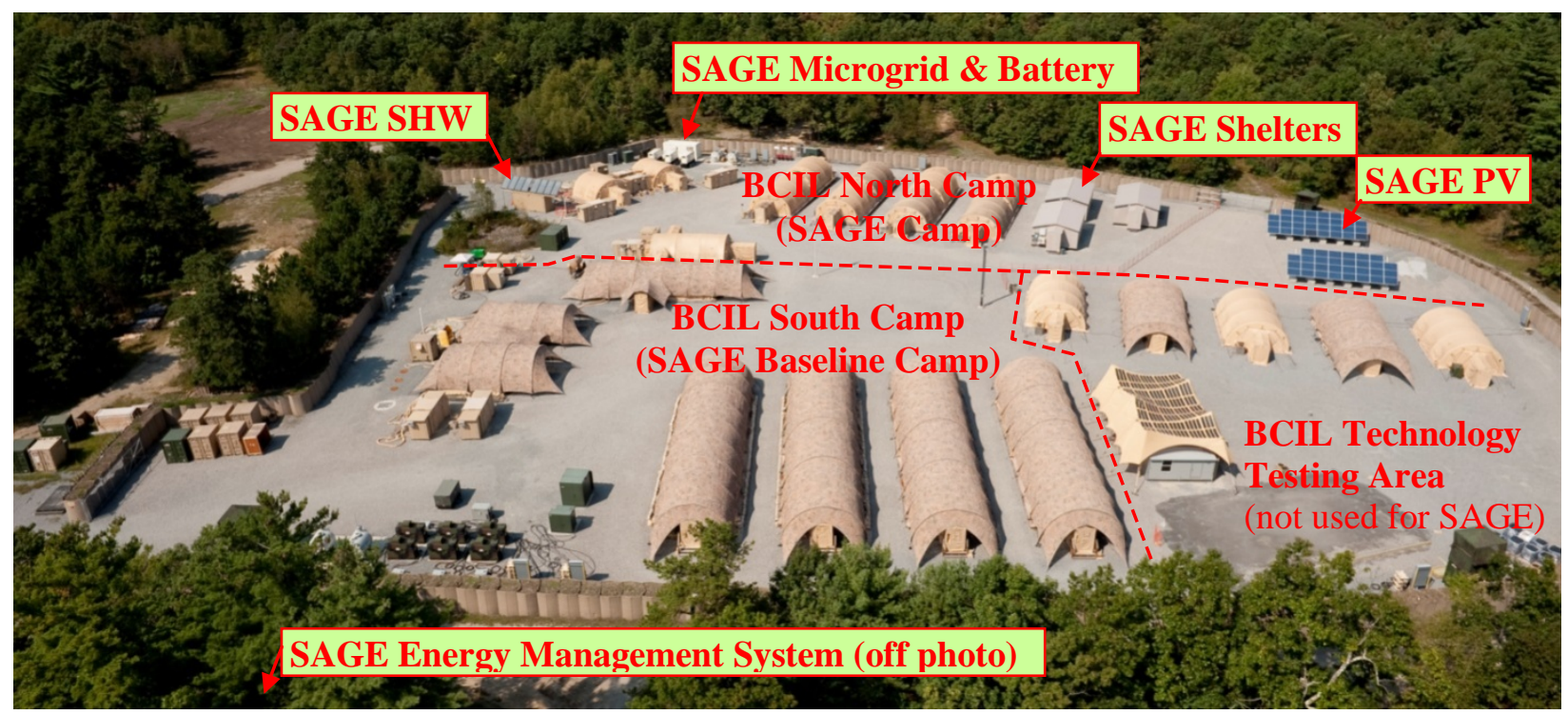

Figure ES-2. BCIL Aerial View

\section{Methodology}

Testing of SAGE technologies was conducted in three phases over a 14-month period starting in January 2012 at a vendor facility in Illinois and ending in March 2013 at the BCIL located in Fort Devens, Massachusetts. BCIL is managed by the U.S. Army Product Manager, Force Sustainment Systems (PM FSS) to simulate existing forward operating bases (FOBs) in locations around the world. It consisted of 10 acres containing two, twin 150-Soldier Force Provider base camps, which allowed SAGE testers to measure and compare fuel consumption between a SAGE base camp and a Baseline camp with standard technology.

Phase 1 tested the microgrid equipment at the vendor's technical center. Phase 2 tested individual SAGE technologies in isolation and integrated at BCIL. Phase 3 focused on evaluating SAGE technologies by testing the integrated system during Soldier occupation over a multi-day training period. Phase 3 also included shelter and SHW performance evaluations. Field test results from Phases 2 and 3 were then used to define accurate models of Baseline and SAGE-improved base camps of various sizes to forecast annual energy and fuel savings for locations across the global climate zones selected by LIA. These locations were selected as areas where geographic combatant commands could potentially operate.

\section{Field Testing}

During field testing, the SAGE camp fuel consumption was compared to the Baseline camp which used spot generation, uninsulated tents with standard Army environmental control units (ECUs), and standard fuel-fired water heaters. The BCIL field-test data included scenarios with no occupancy, simulated occupancy, and actual occupancy by Soldiers. Each of these tests lasted a few days. Table ES-1 summarizes field test results, which demonstrated up to $54 \%$ fuel savings relative to the Baseline when all of the SAGE technologies were implemented. 
Table ES-1. Summary of Field Test Results

\begin{tabular}{|c|c|}
\hline SAGE Technology & Field Tests \\
\hline $\begin{array}{l}\text { All SAGE technologies combined: } \\
\text { (Microgrid + renewable energy source + } \\
\text { energy efficient shelters + SHW system) }\end{array}$ & Up to $54 \%$ annual fuel savings \\
\hline $\begin{array}{l}\text { Microgrid + renewable energy system (PV } \\
\text { array and SHW) }\end{array}$ & Up to $41 \%$ annual fuel savings \\
\hline Microgrid in isolation & Up to $34 \%$ annual fuel savings \\
\hline Microgrid stability & $\begin{array}{l}\text { Microgrid delivered power with stable voltage and } \\
\text { frequency without any power outages or phase distortions. }\end{array}$ \\
\hline Silent run capability & $\begin{array}{l}\text { The energy storage battery system silently powered a } \\
100 \mathrm{~kW} \text { base camp load without generators for two hours. }\end{array}$ \\
\hline Energy efficient shelters & $\begin{array}{l}\text { Shelters demonstrated an average of } 85 \% \text { less energy than } \\
\text { tents and exhibited superior temperature stability. }\end{array}$ \\
\hline Base camp energy management system & $\begin{array}{l}\text { System enabled central monitoring and control of vital base } \\
\text { camp equipment and functions, and could feature } \\
\text { automated load reduction schemes. }\end{array}$ \\
\hline PV array & $\begin{array}{l}\text { The deployed renewable energy power system produced up } \\
\text { to } 12 \% \text { of daily base camp energy needs. }\end{array}$ \\
\hline Solar hot water system & $\begin{array}{l}\text { System reduced the energy consumption of the standard } \\
\text { fuel-fired water heater by an average of } 56.5 \% \text {. (a) }\end{array}$ \\
\hline $\begin{array}{l}\text { (a) Based on Phase } 2 \text { and Phase } 3 \text { field tests; } \\
\text { the occupancy of the Baseline camp was }\end{array}$ & $\begin{array}{l}\text { se } 3 \text { test results were normalized using gallons consumed because } \\
\text { ificantly lower than that of the SAGE camp. }\end{array}$ \\
\hline
\end{tabular}

\section{Modeling Results}

The field-test data were used to construct and calibrate EnergyPlus models of the Baseline and SAGE technologies. EnergyPlus is an U.S. Department of Energy-developed energy modeling software that simulates energy use in buildings. EnergyPlus models building-level heating, cooling, lighting, ventilation, plug loads, other energy flows, and water use. Those energy models were used to estimate the annual fuel use for 150-, 600-, and 5000-Soldier base camps in fourteen climate zones across the world.

The models for all base camp structures, PV arrays, and a SHW system were combined with typical meteorological year statistical weather data for each location. Figure ES-3 shows the fuel savings by location and base camp size using the full suite of SAGE technologies. 


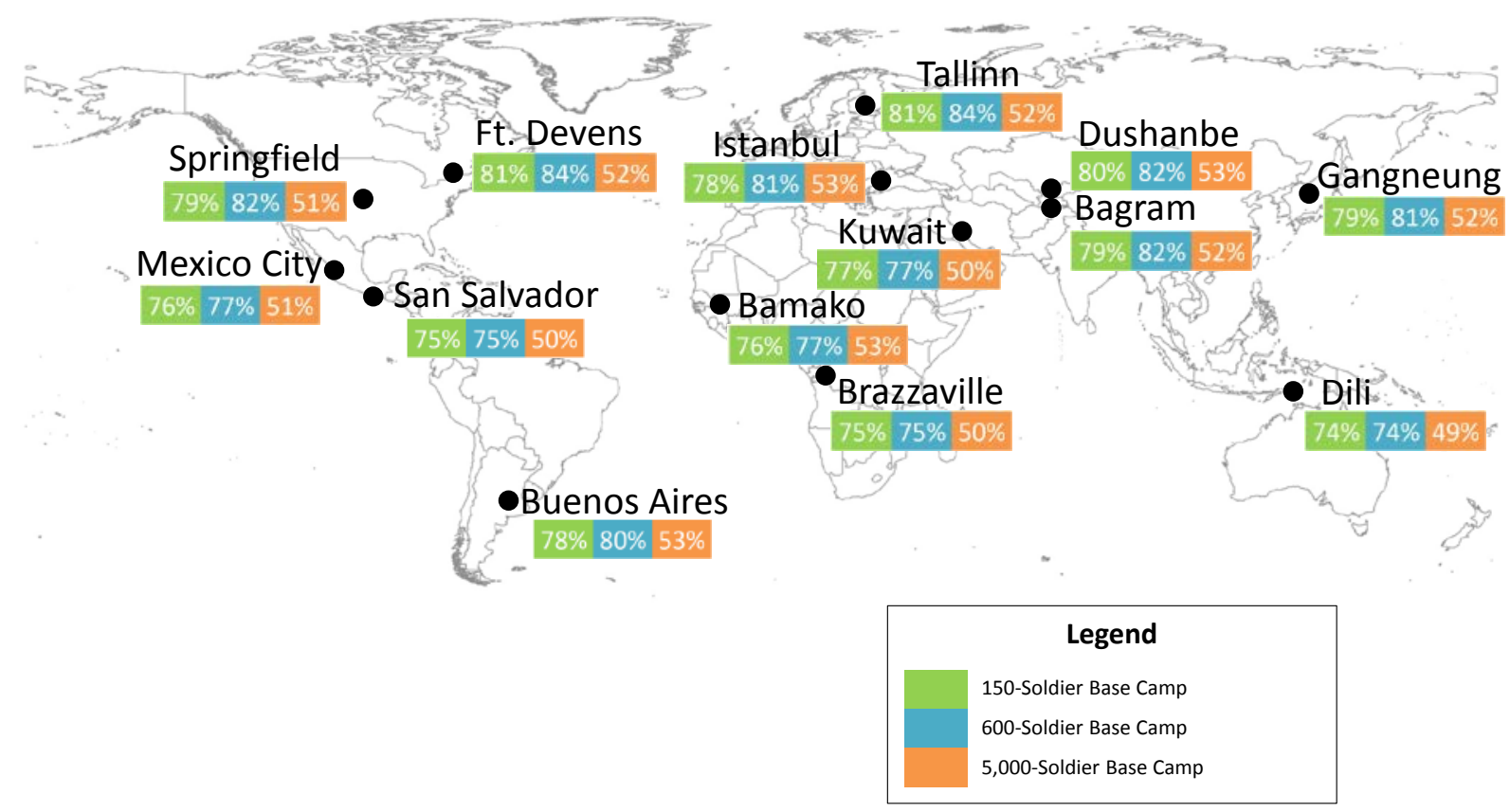

Figure ES-3. Modeled Annual Fuel Savings

The fuel savings for the 150-Soldier base camps (green) ranged from 74\%-81\%. The global fuel savings for 600-Soldier base camps (blue) ranged from $74 \%$ to $84 \%$ and global fuel savings for the 5,000-Soldier base camps (orange) ranged from $49 \%$ to $53 \%$. The savings range for the 5,000 -Soldier base camp is lower because of the more energy-efficient Baseline technology of the larger, more permanent base camps. In other words, the SAGE technologies perform well in small and large base camps, but the Baseline technology performs better in the large base camps, narrowing the gap. The technologies offering the largest fuel savings impact were the energy efficient shelters and smart microgrid. 


\section{Conclusions}

SAGE field testing and modeling indicate that a combination of energy conservation measures, key pieces of fuel-saving equipment, and renewable energy sources can reduce base camp energy consumption significantly. When looked at individually, the most impactful technologies are smart microgrids and energy efficient shelters. Five key conclusions from SAGE testing are summarized in Table ES-2.

Table ES-2. SAGE Conclusions

1. Significant energy savings is readily achievable through deploying two kinds of technologies: a. Technologies to reduce the energy demand (e.g., insulated shelters, building controls)

b. Technologies to improve the efficiency of electricity generation (e.g., microgrids, renewable energy).

2. A combination of energy conservation measures, renewable energy sources, and a smart microgrid can reduce base camp energy consumption by $49 \%-84 \%$ depending on camp size and location. Smart microgrids with energy storage systems supply power with improved voltage and frequency stability, increased grid reliability and longer life of end-use equipment. If microgrids are not possible, right-sizing of existing spot generation has the potential to save significant amounts of fuel annually.

3. Renewable energy systems can reduce base camp energy demand and fuel use. They can be reliably integrated into smart base camp microgrids without harming grid stability or degrading the output of the renewable source. A combination of spot generation with renewable energy sources is generally not recommended.

4. A properly sized SHW heating system can supply a significant fraction of a base camp's water heating requirements using solar energy, thereby saving fuel over standard water heating configurations.

5. A base camp energy management system, equipped with real-time monitoring and control of base parameters, enables central and informed decision making without overburdening the operator with information. Configurable automatic load shedding provides potential for reducing camp energy consumption for normal operations and for unplanned events.

\section{Recommendations}

The following SAGE team recommendations are listed in order of importance when making investment decisions to upgrade or construct more energy efficient base camps:

1. Replace spot generation systems with properly sized power generators that are integrated with smart microgrids. When appropriate, include an energy storage system and a base camp energy management system. When a microgrid is not initially feasible, correctly size the spot generators to meet the actual loads.

2. Replace poorly or un-insulated tents with insulated, energy efficient shelters featuring rightsized high-efficiency ECUs. Add insulation to uninsulated shelters such as wood-framed or concrete block shelters. Include remotely controllable circuits and integration with a base camp energy management system. 
3. Install a well-designed and right-sized sized SHW system in camps with suitable conditions (e.g., expensive energy, remote areas, strong solar resource, high-volume water heating requirements, etc.).

4. Implement a base camp energy management system that features remote control capability of electric loads, reporting, and decision support and can feature automated load reduction schemes.

5. Implement right-sized renewable energy sources suitable for the base camp climate conditions and make sure the renewable energy system can integrate into a microgrid and base camp energy management system.

\section{Way Ahead}

This report is the final deliverable for the SAGE project. Related key deliverables prepared for the SAGE project included:

- Conceptual designs of equipment and buildings, and support the Army in selecting sites on which to deploy the designs,

- Specifications and contracts for the designs, vendor solicitations, procurements, construction/fabrication, field deployments, field testing, and disposition/transfer of equipment and buildings to another Army site, and

- Guide specifications for the key SAGE technologies (Appendix A).

LIA and PNNL, in coordination with the U.S. Army Corps of Engineers Middle East District, began developing specifications and standards for input into the Joint Construction Management System (JCMS), an automated system with a master planning framework and a library of joint contingency standard designs that conform to the Department of Defense Contingency Unified Facilities Criteria. Once SAGE technologies are integrated into contingency construction designs via JCMS, LIA will assist the Program Manager, Logistics Civil Augmentation Program (LOGCAP) in developing contract language to ensure energy efficient measures are incorporated into future base camp contract documents. 


\section{Contents}

Executive Summary . . i

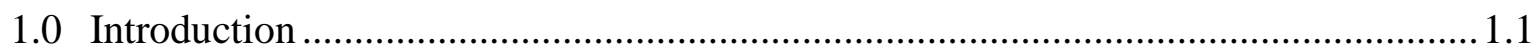

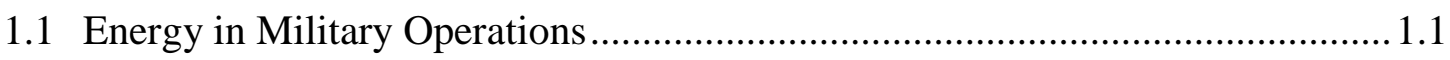

1.2 Project Objectives and Deliverables.............................................................. 1.3

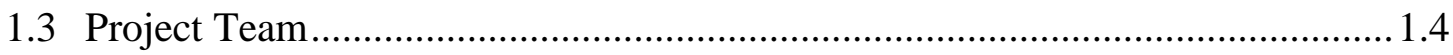

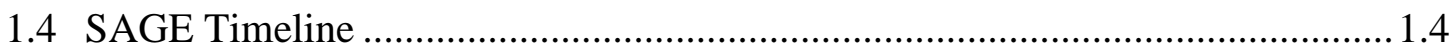

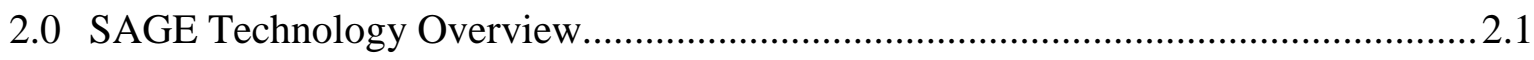

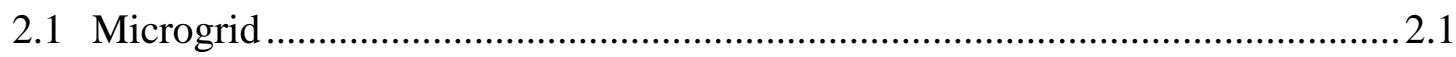

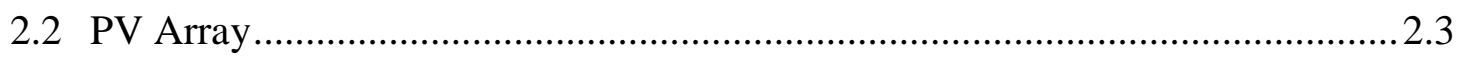

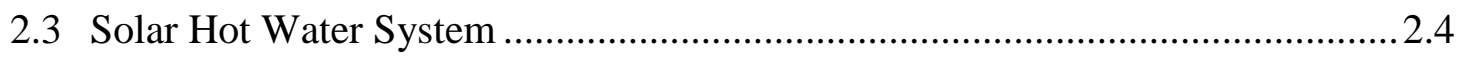

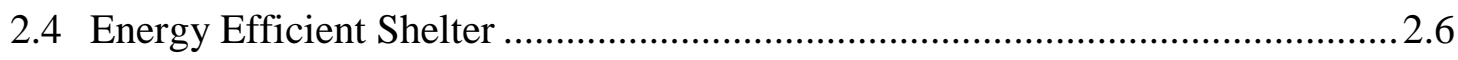

2.5 Base Camp Energy Management System........................................................ 2.7

2.6 Wood-Framed Buildings with Improvements Package.......................................2.8

3.0 Testing Approach, Results, and Analysis.............................................................. 3.1

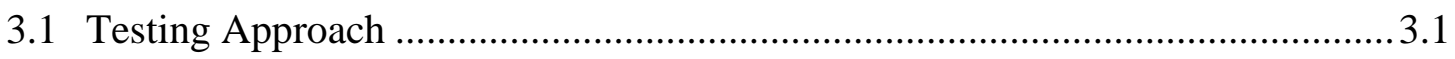

3.2 Test Results and Analysis.......................................................................... 3.5

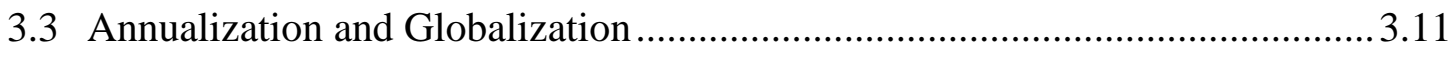

4.0 System Reliability and Durability ......................................................................

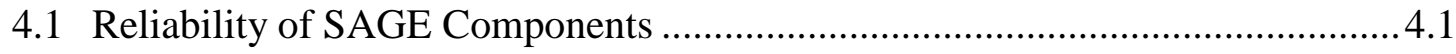

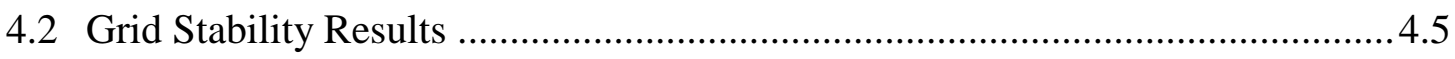

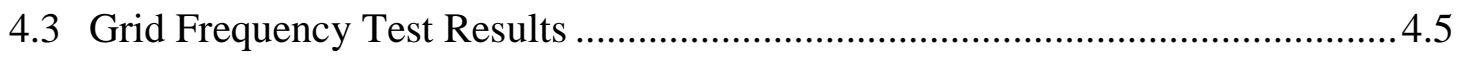

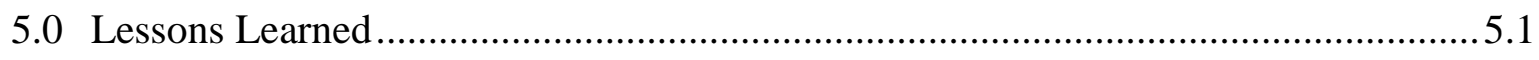

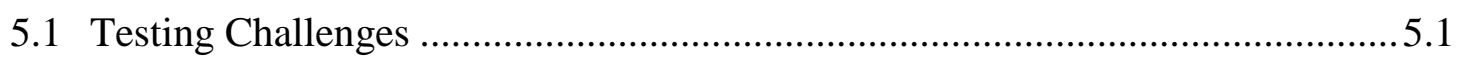

5.2 Recommended Equipment Improvements...................................................... 5.2

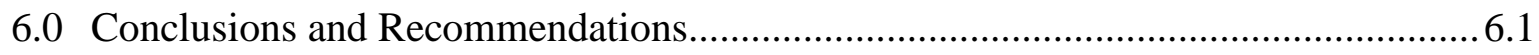

Appendix A SAGE Technology Guide Specifications ................................................... A.1

Appendix B Structure Configuration of 150-, 600-, and 5,000-Soldier Camps ................ B.1

Appendix C Detailed Field Testing Methodology ............................................................ C.1

Appendix D Detailed Modeling and Annualization Methodology .................................... D.1

Appendix E Detailed Field Testing Results................................................................. E.1

Appendix F Acronyms and Abbreviations ................................................................... 


\section{Figures}

ES.1 SAGE Technology Overview Diagram .........................................................ii

ES-2 BCIL Aerial View ................................................................................................iv

ES-3 Modeled Annual Fuel Savings .......................................................................

Figure 2-1. SAGE Microgrid Generators.............................................................. 2.1

Figure 2-2. Generator Efficiency ...................................................................... 2.2

Figure 2-3. Typical Distribution Box.................................................................... 2.2

Figure 2-4. Energy Storage System Container and Battery Strings................................2.3

Figure 2-5. SAGE PV Array ............................................................................ 2.4

Figure 2-6. Solar Hot Water System for the SAGE Prototype ....................................... 2.4

Figure 2-7. Modular, Hard-Walled, Insulated Shelter for SAGE Prototype .................... 2.6

Figure 2-8. Base Camp Energy Management System ............................................... 2.8

Figure 3-1. 24-Hour TIAX Profile vs. 24-Hour Soldier Profile ..................................... 3.8

Figure 3-2. Global Annual Fuel Savings by Camp Size for all SAGE Technologies ..... 3.17

Figure 4-1. Microgrid Frequency Response to a Large Load Transient ......................... 4.6

Figure 6-1. Global Annual Fuel Savings by Camp Size for all SAGE Technologies ........6.2 


\section{Tables}

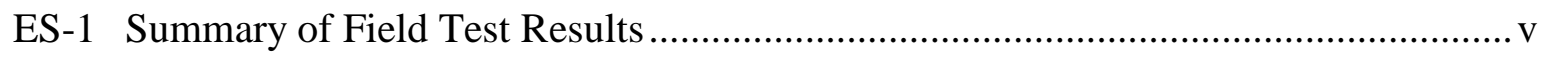

ES-2 SAGE Conclusions ........................................................................................ vi

Table 1-1. Army Fuel Consumption as a Function of Operations Tempo..........................1.1

Table 3-1. BCIL Camp and SAGE Camp Comparison ..................................................... 3.4

Table 3-2. Baseline Camp and SAGE Camp Comparison for all Modeled Camps

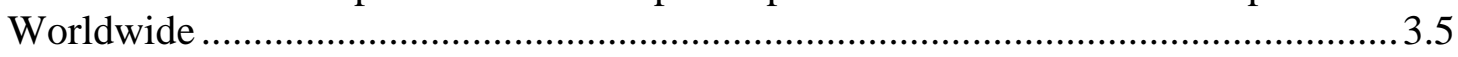

Table 3-3. Phase 2 Fuel Savings by Technology ............................................................ 3.7

Table 3-4. Phase 3 Energy Savings by Technology ........................................................ 3.10

Table 3-5. 150-Soldier Camp Annual Source Energy Savings by Technology (Fort Devens)

Table 3-6. 600-Soldier Camp Annual Source Energy Savings by Technology (Fort Devens)

Table 3-7. 5,000-Soldier Camp Annual Source Energy Savings by Technology (Fort

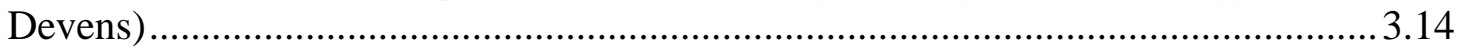

Table 3-8. Annual Source Energy Savings by Structure ………………………….........

Table 3-9. Annual Source Energy Savings by Structure: Baseline vs. Wood-Framed

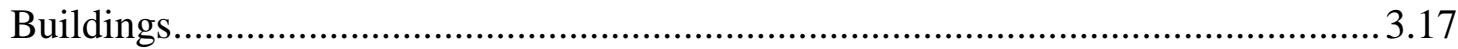

Table 3-10. Detailed Global Fuel Savings for 150-Soldier Camps ......................................

Table 3-11. Detailed Global Fuel Savings for 600-Soldier Camps ..................................... 3.21

Table 3-12. Detailed Global Fuel Savings for 5,000-Soldier Camps ..................................23

Table 4-1. Mean Life of Major Elements in Flat-Plate Collectors .......................................4.4

Table 4-2. Collector Mean-Time-Between-Failure ………........................................... 4.4

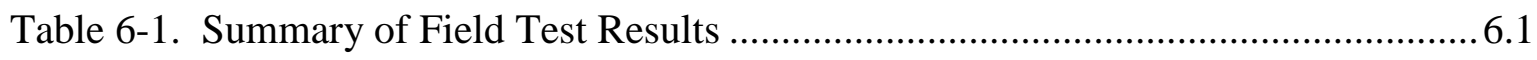

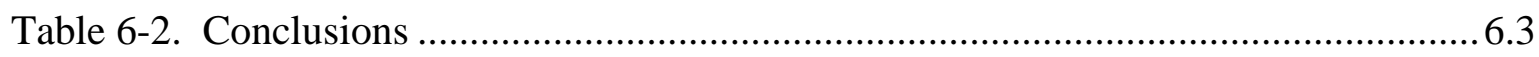





\subsection{Introduction}

\subsection{Energy in Military Operations}

Energy is critical to military operations, and the missions of today's military require substantial and growing amounts of energy which must be supplied through long, vulnerable, and costly supply lines. Recent military engagements have demonstrated the vast energy requirements of war, our reliance on low-cost, abundant energy resources, and an associated culture reflective of our nation's domestic attitudes and expectations towards access to energy. In 2008, the Defense Science Board evaluated and contrasted energy consumption for peace and wartime operations tempo. As detailed in Table 1-1, increased operations tempo had a corresponding increase in energy consumption, but the relative increase of energy consumption across different energy consumers changed dramatically. For instance, generator fuel consumption during a wartime operations tempo increased by more than tenfold and became the single largest source of energy consumption within the Army, whereas non-tactical energy consumption remained constant.

Table 1-1. Army Fuel Consumption as a Function of Operations Tempo

\begin{tabular}{lcccc}
\hline $\begin{array}{c}\text { Energy } \\
\text { Consumers }\end{array}$ & $\begin{array}{c}\text { Wartime Optempo, } \\
\text { million gallons }\end{array}$ & $\begin{array}{c}\text { Percent of } \\
\text { Total }\end{array}$ & $\begin{array}{c}\text { Peacetime Optempo, } \\
\text { million gallons }\end{array}$ & $\begin{array}{c}\text { Percent of } \\
\text { Total }\end{array}$ \\
\hline Non-Tactical & 51 & $5 \%$ & 51 & $18 \%$ \\
Combat Vehicles & 162 & $15 \%$ & 30 & $10 \%$ \\
Tactical Vehicles & 173 & $16 \%$ & 44 & $15 \%$ \\
Combat Aircraft & 307 & $29 \%$ & 140 & $48 \%$ \\
Generators & 357 & $34 \%$ & 26 & $9 \%$ \\
\hline Total & $\mathbf{1 , 0 5 0}$ & $\mathbf{1 0 0 \%}$ & $\mathbf{2 9 1}$ & $\mathbf{1 0 0 \%}$ \\
\hline
\end{tabular}

Such increases in demand for energy strained logistical support and budgets. To avoid similar constraints in future engagements, the Army identified the need to address energy consumption via a spectrum of approaches and technologies but avoid the temptation to produce "one size fits all" solutions, since there is no single or simple suite of actions that will produce the desired outcome.

To address methods and means to reduce energy consumption with a holistic approach, the Smart and Green Energy (SAGE) project team explored the following categories of energy improvements:

1. energy conservation measures (e.g., building insulation, building controls, etc.)

2. grid efficiency and security technologies (e.g., improved generators, energy storage, etc.)

3. renewable energy systems (e.g., solar collectors for power and for hot water).

When weighing the relative merits of these opportunities, the Army could benefit from holistically assessing these approaches in unison rather than focusing on them individually, as 
there are interactive effects that could be considered. For instance, the quantity and associated cost of using renewable energy to supply a large fraction of base camp energy is significant. However, if base camps are more inherently efficient, then lower levels of renewables are required to accomplish the same function and the use of renewables becomes increasingly more practical and affordable.

In short, the three focus areas of energy conservation, energy efficiency, and renewable energy are fundamentally different, but are interconnected, and the approach taken by SAGE was to find the optimal balance between these three energy saving avenues.

\subsubsection{Operational Energy}

In the Duncan Hunter National Defense and Authorization Act for Fiscal Year 2009, operational energy was defined as the energy and associated systems, information, and processes required to train, move, and sustain forces and systems for military operations. Operational energy has three components: Soldiers, vehicles/platforms, and basing. The SAGE for Base Camps project addressed the basing component of operational energy by seeking ways to reduce annual base camp fuel consumption.

\subsubsection{Today's Baseline Conditions}

Currently, base camp electric energy is mostly generated with traditional combustion engine generators. Smaller forward operating bases (FOBs) typically use spot generation, a practice that assigns a single generator to a single load or a small group of loads. Loads or load groups are assigned to a single generation until all loads are provided with power. Since there is no electric grid connecting loads to a common set of generators, each generator must be sized to meet the maximum power demand of its load even if these peak loads are infrequent. As a result, generators typically operate with notable levels of excess generation capacity. Since generator efficiency is proportional to its load level, this practice of under-loading generators leads to poor fuel economy and increased generator maintenance due to increased run hours and wet stacking, a condition resulting from poor fuel combustion.

\subsubsection{Energy Logistics and Challenges}

In recent years, military engineers and logisticians have encountered several operational energy challenges. These include asymmetric threats to logistics convoys and infrastructure, increased global competition for limited oil supplies, heightened and volatile costs for petroleum resources, and growing concerns regarding climate change. Highly diverse Army operations span a variety of operating environments, and often include vulnerabilities in energy infrastructure such as fuel transport and shortfalls in power generation. Further, while petroleum-based energy is convenient for ease of handling and global availability, it is vulnerable to price volatility. 


\subsubsection{Addressing Operational Energy Challenges through SAGE}

In 2009, the Army's Senior Energy Council issued an Army Energy Security Implementation Strategy that included five strategic energy security goals: 1) reduce energy consumption, 2) increase energy efficiency across platforms and facilities, 3) increase use of renewable/ alternative energy, 4) assure access to sufficient energy supplies, and 5) reduce adverse impacts on the environment. SAGE addresses each of these goals.

Smart microgrids ${ }^{1}$ have the potential to generate electric power more reliably and with less fuel cost when compared to spot generation or standard, non-smart electric grids. Furthermore, when microgrids replace spot generation, fuel savings and power quality improvements are virtually guaranteed, provided that operators are properly trained and the system is maintained regularly. The addition of an energy storage system further increases power quality and reliability. When renewable energy sources are used to supplement standard generators, fuel savings are more pronounced. These aforementioned technologies address the fundamental problem from the power generation side, otherwise known as the "supply side."

SAGE also addresses the energy "demand side" of base camps by replacing tents with insulated hard-walled shelters and with a base camp energy management system. Uninsulated tents require large quantities of energy for space heating and cooling. While insulated tent liners improve this shortcoming, a properly insulated, hard-walled shelter with reduced energy loss from air leaks can be highly energy efficient and more comfortable for occupants. A base camp energy management system is an automated tool that allows a base camp energy manager to easily plan, monitor and manage operational energy. The system informs the energy manager in real time how energy is being used throughout the base camp. System controls allow the energy manager to use demand-side management to regulate unused or noncritical heating/cooling units or to turn off unused space lighting. These energy conservation measures directly contribute to reduced energy consumption.

\subsection{Project Objectives and Deliverables}

The primary objective of the SAGE project was to demonstrate a 'proof-of-concept' as to how commercially available energy efficient and renewable energy technology can be used to reduce base camp energy consumption. The demonstrated technologies included a commercially sourced smart microgrid suitable/scalable for a 150-Soldier to 6,000-Soldier base camp. Also evaluated were insulated hard-walled shelters, solar hot water (SHW) heating systems, photovoltaic (PV) arrays, and a base camp energy management system in order to demonstrate a holistic approach to energy savings.

Additional objectives included reducing base camp fuel consumption by 30-60\%, reducing generator runtime and maintenance intervals, demonstrating a 'silent run' capability, integrating renewable energy sources into a microgrid, and allowing automated energy management and

\footnotetext{
${ }^{1}$ Smart grids include automatic load and generation controls, active and automated devices that regulate grid health, and sensors for data gathering. Non-smart grids operate without these controls or sophisticated data gathering systems.
} 
reporting. The objectives did not include evaluating the differences between manufacturers such as generator manufacturers, or between types of systems such as lead acid vs. lithium ion batteries, nor did they include determining the optimum designs of the individual or integrated technologies.

SAGE project deliverables include design standards and specifications (Appendix A) that the U.S. Army Corps of Engineers (USACE) could input to the Joint Construction Management System (JCMS) and that the Program Manager, Logistics Civil Augmentation Program (PM LOGCAP) could integrate into future contract language; this final report; and an organized set of raw data.

\subsection{Project Team}

The SAGE project was executed via a partnership of the U.S. Army Logistics Innovation Agency (LIA) and the Pacific Northwest National Laboratory (PNNL).

\subsubsection{Army Logistics Innovation Agency}

The LIA is the Field Operating Agency of the Office of the Deputy Chief of Staff of the Army, G-4. The Agency provides innovative policy, process, program, and capability solutions that enhance logistics operations. LIA continually examines Army logistical capabilities and processes to identify ways to make it more effective and/or efficient. As such, LIA was well positioned to lead the SAGE project to reduce fuel consumption at Army base camps.

\subsubsection{Pacific Northwest National Laboratory}

PNNL is a U.S. Department of Energy (DOE) research organization operated by Battelle Memorial Institute. PNNL's mission is to transform the world through courageous discovery and innovation that will enable the world to live prosperously, safely, and securely. PNNL's electricity infrastructure and buildings research includes energy efficiency strategies, renewable energy systems integration, and efforts to transform the nation's power grid and microgrids to be clean, efficient, reliable, and resilient.

\subsection{SAGE Timeline}

\subsubsection{Planning}

SAGE's detailed planning began in September 2010 to define the range of potential technologies for demonstration and to select a demonstration site. The technology and site selections proceeded in parallel. The technology selection process focused on identifying generic grid technologies and representative ancillary technologies, such as renewables and energy efficient shelters that could demonstrate $30 \%$ to $60 \%$ energy savings and that could be quickly deployable. The technology selection process also included informational discussions with specific technology providers. In addition, discussions were begun with several LIAidentified stakeholders so that the technologies to be selected by SAGE would be more 
compatible with - and attractive to- the existing military design, construction, and operations organizations. These organizations included the USACE managing the Theater Construction Management System (TCMS), which later evolved to JCMS, and the USACE Army Prime Power School. A conceptual design was developed in February 2011 for internal discussions on potential SAGE technologies. The conceptual design included the placement of SAGE technologies on a generic FOB 150-Soldier base camp plan and a generic product specification, all to illustrate to stakeholders the technologies and their physical integration in the field.

LIA evaluated several U.S. Department of Defense sites for installing and demonstrating the SAGE technologies to be selected. Middle Eastern sites were considered but the siting focused on the United States to make SAGE more accessible to U.S.-based military command and energy management personnel. The BCIL that was under construction on Fort Devens, Massachusetts, was selected in April 2011 because BCIL's planned design and use supported several SAGE goals. BCIL's construction was to be completed for Soldier occupancy in August 2011. BCIL's planned installation of a pair of identical Force Provider 150-Soldier base camps could serve as SAGE's Baseline and test camps. BCIL planned for a high number of Soldier occupancies that would enable SAGE to be demonstrated in more realistic field conditions. Also, BCIL planned an extensive energy- and water-metering and data-acquisition system that SAGE could employ for its testing. The field tests at BCIL were intended as a proof-of-concept that could then be further explored via detailed modeling in a wide variety of global locations in camp sizes up to 5,000 inhabitants. The tests were not conducted to evaluate the Force Provider camp standard itself, but to use the camps as a surrogate for existing and planned camps around the world.

\subsubsection{Phase 1 - Microgrid and Storage Factory Tests}

Phase 1 testing occurred from January to February 2012 at the Caterpillar Technical Center in Mossville, Illinois. The objective was to verify that the SAGE smart microgrid with energy storage system worked as designed and was capable of incorporating a renewable energy system. Testing was accomplished by Caterpillar and witnessed by both PNNL and LIA personnel. After the successful conclusion of Phase 1 testing, the microgrid, energy storage system and associated equipment were shipped to Fort Devens, Massachusetts.

\subsubsection{Phase 2 - Field Tests at BCIL with Load Bank}

Phase 2 testing occurred from April through August 2012 at the BCIL at Fort Devens, Massachusetts, with an additional SHW test performed in October 2012. Test objectives included acceptance testing of the turnkey systems fabricated and installed by the vendors, which included the smart microgrid and energy storage system, the PV arrays, the energy efficient shelters, and the SHW system. Testing also included the base camp energy management system developed and installed by PNNL. During this phase, each technology was tested in isolation from the other technologies. The microgrid, energy storage system, and PV array were then tested with a load bank (a device to simulate electrical loads) to evaluate system performance 
with load profiles that PNNL developed from the TIAX ${ }^{2}$ report, which included typical load profiles for common base camp buildings.

\subsubsection{Phase 3 - Field Tests at BCIL with Soldiers}

Phase 3 testing occurred at the BCIL from October 2012 to March 2013 and consisted of two tests with different objectives. The objective of the first test was to examine all SAGE technologies simultaneously in one 150-Soldier base camp with Soldier occupancy, while simultaneously comparing its performance to an occupied, Baseline 150-Soldier base camp. The second test occurred between November 2012 and March 2013 and involved collection of data during the fall to evaluate the performance of the SHW system, energy efficient shelters, and standard uninsulated tents equipped with fuel-fired heaters.

Field data collection was neither long term nor continuous because of low Soldier occupancies and delays in BCIL's data acquisition system. The actual Soldier occupancies were intermittent and the number of Soldiers for each occupancy was typically lower than the 150Soldier capacity of one BCIL base camp. In addition, the Soldiers and their activities could not be distributed between the two base camps in a way that would help balance the electrical loads between the two base camps. The installation and commissioning of BCIL's water and electrical metering system was fully completed late in SAGE's testing.

\subsubsection{Post-Testing Activities}

The test data gathered from Phase 1 and Phase 2 tests were used to develop EnergyPlus software models for all buildings in 150-, 600-, and 5,000-Soldier base camps for the Baselines and the SAGE versions. These models were used to generate creditable power profiles in fourteen different global locations along with power generation profiles for renewable energy sources and camp power generation equipment. Modeling and simulation established annual energy and fuel needs for Baseline configuration camps as well as for camps with various levels of SAGE technology implementation.

Other SAGE post-testing activities focused on developing standards and specifications for input into the (USACE's) JCMS and LOGCAP. In addition, SAGE test results and raw data were provided to the U.S. Army Materiel Systems Analysis Activity (AMSAA) for independent analysis that supported the LIA cost-benefit analysis that will be reviewed by the Deputy Assistant Secretary of the Army for Cost and Economics. Work also began on certifying the SAGE energy management system for possible adoption across the Army. Lastly, post-testing activities included shipping SAGE equipment from the BCIL to the Contingency Basing Integration Technology and Evaluation Center (CBITEC) in Fort Leonard Wood, Missouri, as well as developing this report and organizing raw data for distribution.

\footnotetext{
${ }^{2}$ TIAX. 2006. Force Provider Electrical Survey, Analysis, and Market Investigation - Final Report. TIAX Ref. No. D0324, TIAX, LLC, Lexington, Massachusetts.
} 


\subsection{SAGE Technology Overview}

\subsection{Microgrid}

\subsubsection{Generators and Generator Controller}

The SAGE microgrid was composed of three diesel or JP-8 commercial Caterpillar XQ100, 100-kilowatt (kW) internal combustion engine generators. Each generator was capable of drawing fuel from an internal or external tank. The generators were connected with proprietary computerized controllers that allowed parallel operation and load sharing between generators. An existing legacy $60 \mathrm{~kW}$ Tactical Quiet Generator (TQG), retrofitted with the same controller, was connected to the microgrid and operated seamlessly with the three commercial generators. The addition of the retrofitted $60 \mathrm{~kW} \mathrm{TQG}$ also illustrated a key feature of the microgrid in that the number and size of the generators did not have to be fixed as long as the same controller was being used. The controller employed nonproprietary communication protocols that enabled communication between the generators and the base camp energy management system. The generators are shown in Figure 2-1.

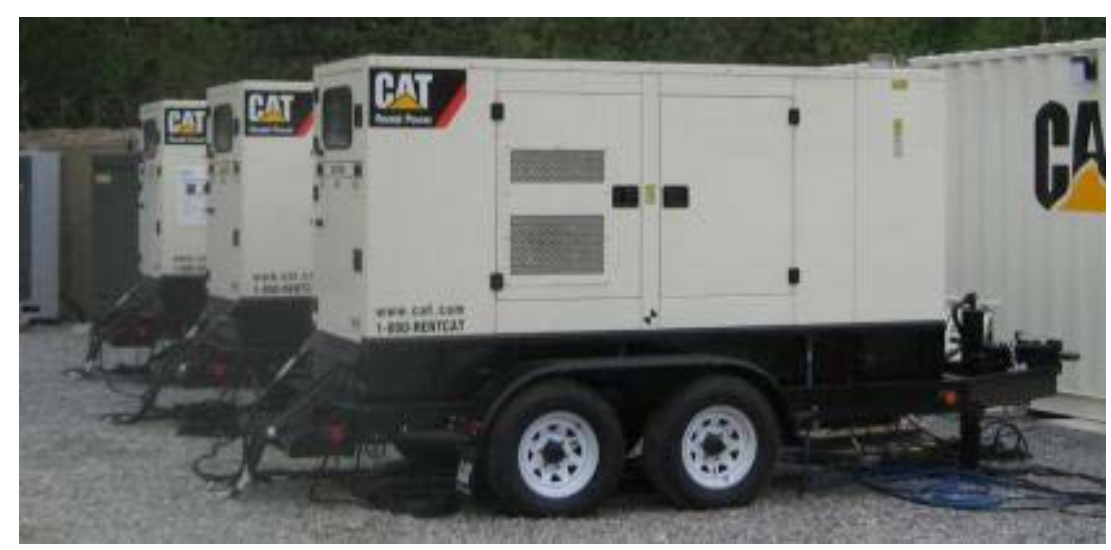

Figure 2-1. SAGE Microgrid Generators

A critical feature of a microgrid is generator load sharing and load sensing. Generators with intelligent controls and load sharing/load sensing capabilities can automatically share the aggregate camp load across multiple generators and adjust the number of generators engaged based on the load. This key feature is not possible with spot generation and is largely responsible for the microgrid fuel savings because the individual generators run more efficiently at higher loads and activate only when necessary. With spot generation, individual generators often run at loads significantly lower than maximum rated capacity, which is detrimental to generator efficiency and lifetime.

Generator efficiency can be expressed as a percentage of the system input energy (i.e., fuel) converted to useful output (i.e., electric energy). Typical diesel generator efficiencies vary from $22 \%$ to $41 \%$. A critical factor in determining generator efficiency is the load imposed on the generator as a percentage of the generator capacity (e.g., $25 \mathrm{~kW}$ on a $100 \mathrm{~kW}$ generator or $25 \%$ 
load). In most instances, a generator performs most efficiently when loaded between $80 \%$ and $95 \%$ of its rated power. This performance depends upon the generator make, model and intended operation (e.g., prime power, backup power, etc.). The efficiency of the unit will decrease as the imposed load decreases away from the optimal load level. Furthermore, due to internal friction and a general lack of sophisticated subsystems, smaller diesel generators generally are less efficient than larger units. This is illustrated in Figure 2-2.

\section{Generator Efficiency}

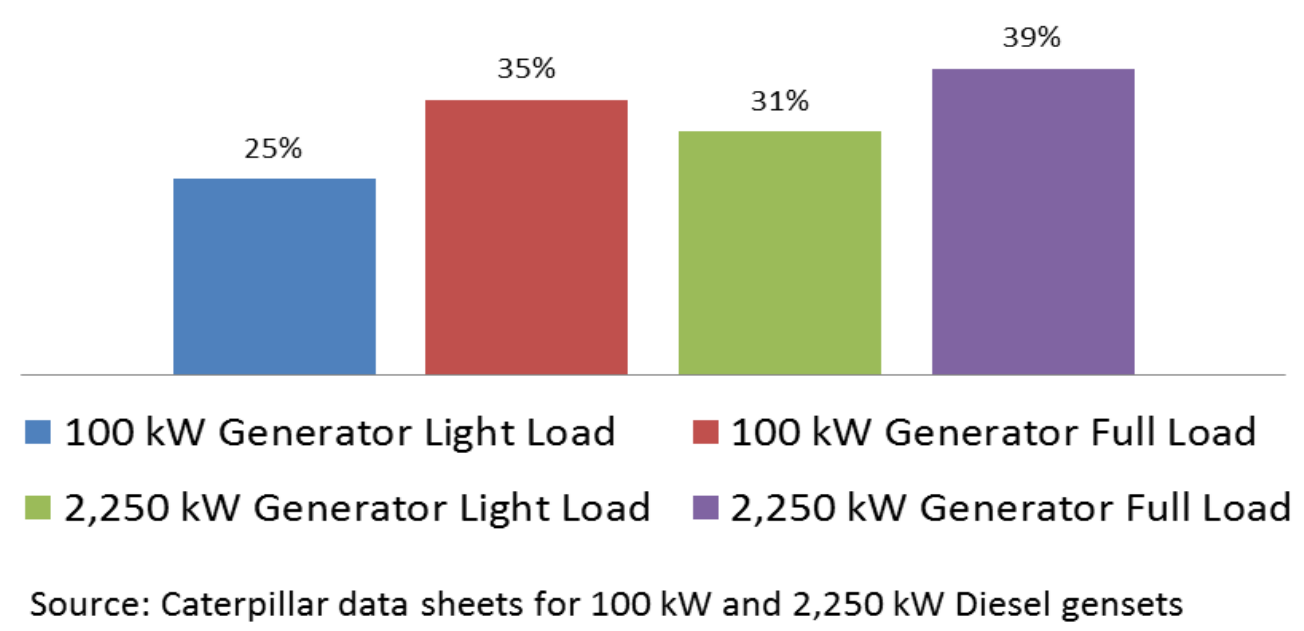

Figure 2-2. Generator Efficiency

\subsubsection{Power Distribution}

The SAGE smart microgrid generated and distributed electric power in three-phase configuration at 208 volts (line-to-line)/120 volts (line-to-neutral) for the 150-Soldier SAGE base camp. The SAGE-enabled base camp did not use transformers because the generators produced power at the supply-side voltage and power distribution was accomplished via LEX Products PowerHOUSE ${ }^{\mathrm{TM}}$ portable distribution boxes (shown in Figure 2-3). Cabling was rated for the transmitted power.

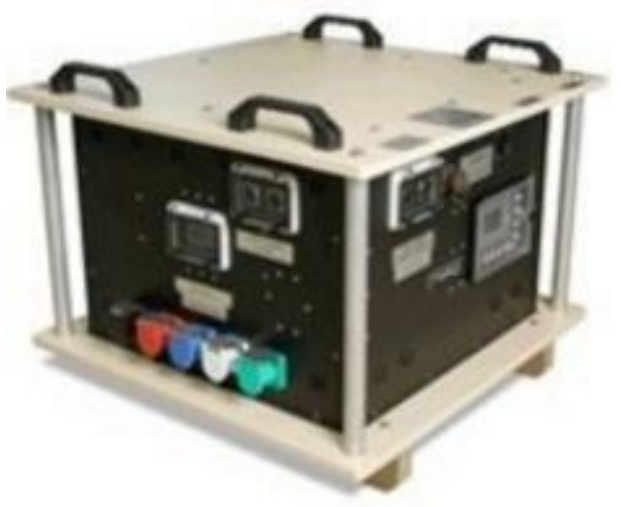

Figure 2-3. Typical Distribution Box 


\subsubsection{Energy Storage}

The SAGE energy storage system (see Figure 2-4) stored electric energy that was produced by the generators and could release this energy back to the microgrid when it was needed. It was designed to silently power a reduced base camp load of $100 \mathrm{~kW}$ for two hours or a larger base camp load of $250 \mathrm{~kW}$ for 20 minutes. The SAGE energy storage system consisted of sealed lead acid batteries and a bidirectional inverter housed inside a 20 -foot ISO freight container with a heating, ventilating, and air conditioning (HVAC) unit. The bidirectional inverter managed and monitored battery charging, discharging, and health while the HVAC system maintained conditions for battery health and longevity. While the SAGE energy storage system prototype was placed adjacent to the generators to simplify testing at the BCIL, this configuration may not be desirable or optimal for overseas base camps. To economically demonstrate the SAGE 'proof of principle,' the SAGE prototype used less-expensive lead acid batteries. Other battery chemistries (e.g., lithium ion) or storage techniques (e.g., flywheel, hydrogen, etc.) can be adopted to meet the energy storage requirement.
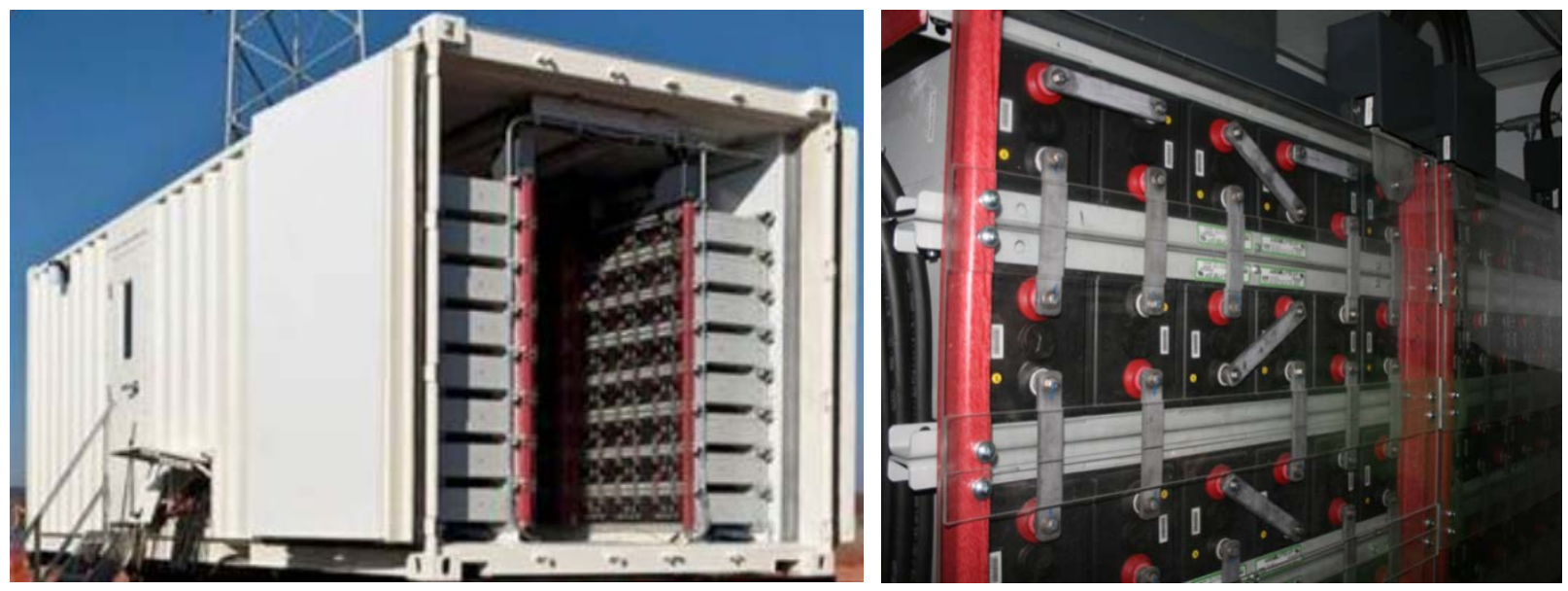

Figure 2-4. Energy Storage System Container (left) and Battery Strings (right)

\subsection{PV Array}

SAGE featured two PV arrays that converted solar energy into electrical energy. The SAGE array consisted of individual PV panels strung together into arrays as illustrated in Figure 2-5. Inverters were used to convert direct current (DC) power produced by the array to alternating current (AC) power, which is the form of power used by most base camp equipment. While the energy produced by an array is contingent on the availability of solar energy, the energy gained is freely available, renewable, and does not impose significant maintenance costs.

The PV modules employed by SAGE were $13.7 \%$ efficient and had a combined power output of $12.9 \mathrm{~kW}$ (6.5 kW per array). The $6.5 \mathrm{~kW}$ size was selected to represent SAGE's conceptual electrical load estimate for a hypothetical $20 \mathrm{ft} \times 30 \mathrm{ft}$ insulated billet building with 22 occupants. Each array was anchored to the ground with six concrete blocks and had a $44 \mathrm{ft} \times 10 \mathrm{ft}$ footprint. 


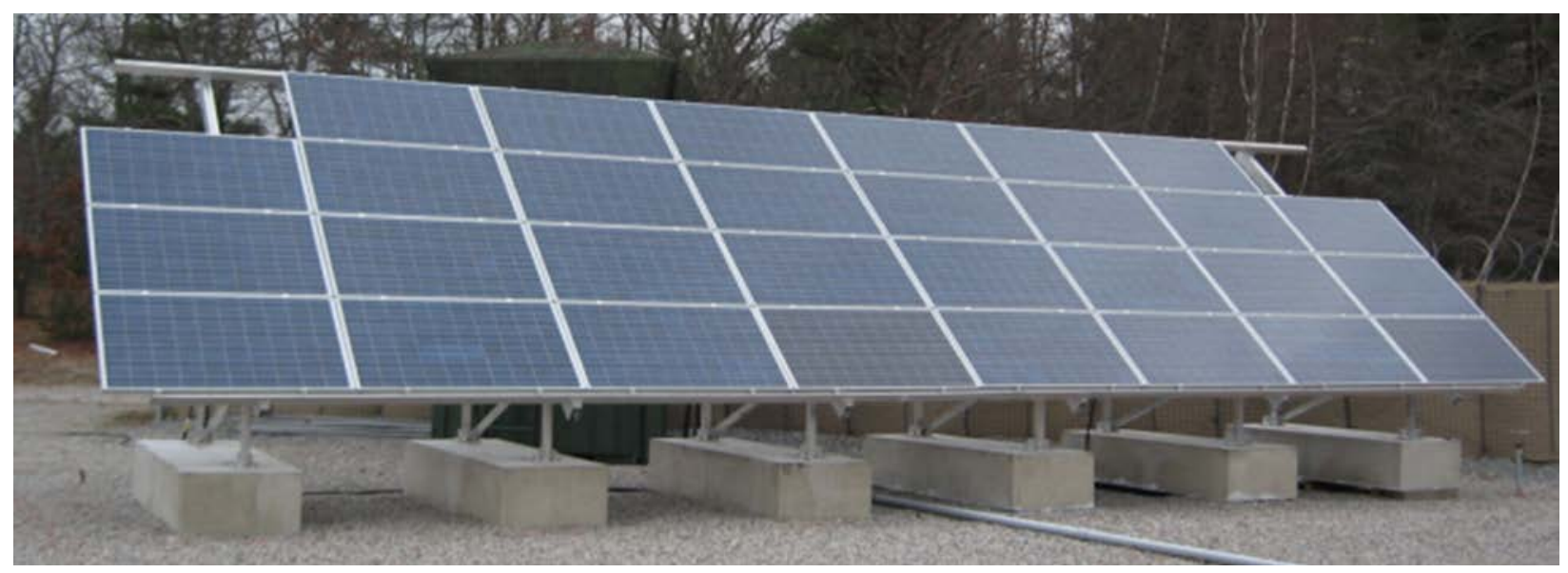

Figure 2-5. SAGE PV Array

PV arrays are highly scalable, and in-theater capacity could be increased until space or grid integration limits are reached. Furthermore, the use of higher efficiency modules could increase the power output per unit area dedicated to an array. Other renewable energy systems such as wind and micro-hydro can also be integrated into a SAGE-style microgrid.

\subsection{Solar Hot Water System}

SAGE featured an integrated SHW system consisting of collector panels, storage tank, loadside immersed heat exchanger and electronics as illustrated in Figure 2-6. The SHW system preheated potable water that was piped to a standard Army fuel-fired water heater (a.k.a. Army Water Heater (AWH) Model 400) that heats the water as needed to meet target temperatures for shower facilities. By using solar energy to preheat the water prior to the AWH 400, a SHW system could reduce or eliminate fuel use for water heating.
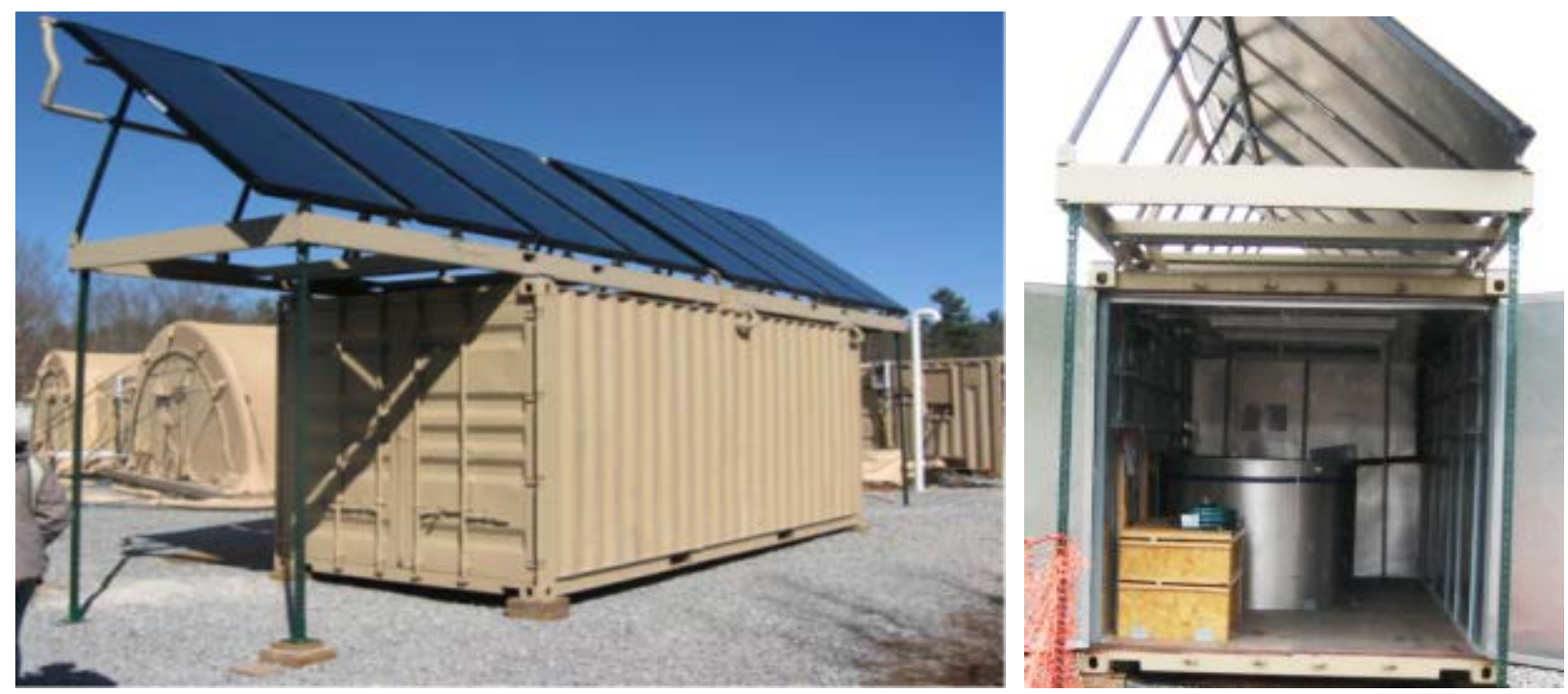

Figure 2-6. Solar Hot Water System for the SAGE Prototype 
The SHW system selected by SAGE for this demonstration was an active, indirect (closed loop) drain-back ${ }^{3}$ system using untreated water as the heat transfer fluid. The solar collection part of the SHW system operated according to the following logic:

1. The SHW system controller constantly monitors the temperature of the collector panel (located on the roof of the container) and the temperature of the fluid inside the storage tank (located inside the SHW container).

2. When the collector panels are warmer than the storage tank by an amount set by the SHW vendor, the system controller starts the water pumps to circulate the fluid through the panels.

3. As the fluid moves through the collector panels, the fluid absorbs the solar energy. The fluid then drains by gravity from the collector panels into the storage tank.

4. When the collector's temperature drops below the differential temperature, or when the tank temperature reaches 160 degrees Fahrenheit $\left({ }^{\circ} \mathrm{F}\right)$, the controller turns off the pumps.

The transfer of solar heat into the potable water system operated according to the following logic:

1. When a base camp shower turns on, the base camp potable water pumping system moves potable water from the base camp well and/or the base camp water storage to the shower.

2. The base camp pumping system first routes the water through a copper coil heat exchanger ${ }^{4}$ in the SHW storage tank and thereby the potable water absorbs whatever tank heat is available.

3. The base camp pumping system then routes the water to the base camp hot water heater which is a fuel-fired instantaneous boiler, and then on to the base camp showers and sinks.

4. When the solar-warmed water entering the boiler is cooler than the boiler's preset target temperature, the boiler's controller turns on the boiler to add heat to the water.

The SAGE SHW system was designed so that all its components could be securely stowed for shipping in a standard ISO 20-foot shipping container; the SHW vendor indicated that their next-generation SHW would work with one 10-foot ISO container. For deployment at BCIL, the vendor removed the solar collectors and support frame out of the container and installed them on

\footnotetext{
${ }^{3}$ Active (a.k.a. forced) is "a system which utilizes mechanical means to circulate fluid through the collector." Indirect (a.k.a. closed) is "a system that provides separation between the collector fluid and the potable water." In this type of system, there are two fluids: one heat transfer fluid that flows through the collector and the other fluid (here defined as potable water, but could be any medium) passes through another component, typically a heat exchanger transferring the heat. Drain-back refers to "a system in which the fluid in the solar collector is allowed to drain back to storage whenever solar energy is not being collected; i.e., the fluid circulating pump is not operating." Excerpts from ASHRAE Standard 95-1987 (reaffirmation of 95-1981 with minor editorial revisions), Methods of Testing Thermal Performance of Solar Domestic Water Heating Systems, American Society of Refrigerating and Air-Conditioning Engineers., Inc. Atlanta, GA. 1988.

${ }^{4}$ Defined as follows: a "load-side heat exchanger is used to remove energy from a hot storage tank (usually unpressurized) by flowing water through the heat exchanger before going to the load." Farrington, R.B. and Bingham, C.E. Testing and Analysis of Load-Side Immersed Heat Exchangers for Solar Hot Water Systems. October 1987. Solar Energy Research Institute.
} 
the top of the container. The tank, internal piping, and electronics remained as positioned inside the container for operation.

The SAGE SHW system was constructed to provide slightly more than 400 gallons of hot water $^{5}$ or 50 showers per day for the specific purposes of the SAGE demonstration. However, SHW systems and components are available to scale up to any practical size.

\subsection{Energy Efficient Shelter}

The SAGE base camp featured three modular, insulated hard-walled shelters sized to match the $20 \mathrm{ft} \times 32 \mathrm{ft}$ footprint of a standard air-beam tent. These buildings were intended to demonstrate the energy savings that could be achieved by incorporating a basic set of commercial off-the-shelf (COTS) energy efficiency technologies. The shelters' floor, wall, and roof panels were rated at an R-25 insulation value. Each shelter included a high-efficiency, $3 \mathrm{~kW}$ heat/cool pump with a Seasonal Energy Efficiency Ratio of 13. Each shelter included a pair of air ventilation units to provide fresh air for the occupants and a heat exchanger that minimized the energy lost in exhausting air from the shelter. Shelter lighting was accomplished via low-power light-emitting diode (LED) lamps ( $150 \mathrm{~W}$ total load per shelter). Electric breakers and thermostats were remotely controllable by the base camp energy management system, allowing manual and automated load management. For example, testers could remotely turn off $50 \%$ of the convenience outlets and change the thermostat temperature set point by $5^{\circ} \mathrm{F}$. When these types of demand reduction actions are followed regularly, significant energy savings can be achieved. Figure 2-7 shows one SAGE shelter with its heat pump unit and the controllable electrical circuit breaker box.

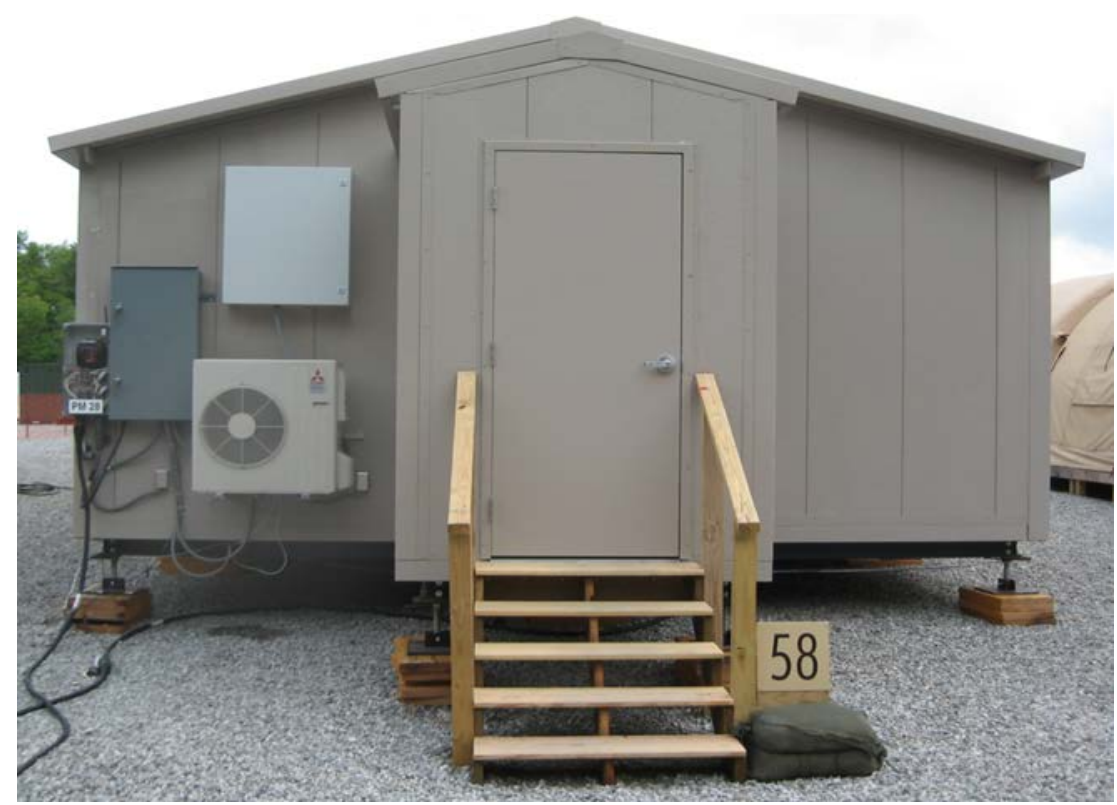

Figure 2-7. Modular, Hard-Walled, Insulated Shelter for SAGE Prototype

\footnotetext{
51.5 gallons/minute $\times 8$ minutes/shower $\times 50$ showers $=600$ gallons, $\times 2 / 3$ (hot water/total water $)=400$ gallons .
} 


\subsection{Base Camp Energy Management System}

The SAGE base camp included a base camp energy management system prototype built upon a federally developed software system known as the Decision Support for Operations and Maintenance (DSOM) system. DSOM, which is used by power plants and building automation systems, was selected because it had an existing open, flexible configuration and capability that could be easily adapted for energy management at base camps. In addition to collecting metered data from the SAGE equipment, the system also communicated with the BCIL's metering and data acquisition systems over a local area network. This system was used to demonstrate the energy savings that could be achieved via automated thermostat setbacks and outlet controls, as well as the utility of a central monitoring system.

To provide base camp energy information to decision makers, a dashboard was developed that displayed near-real-time information for instruments, equipment, and sensors. Information presented included microgrid parameters (power, efficiency, fuel levels, etc.), tent and shelter temperatures, SHW data, and meteorological data. The system also featured direct manual and automatic control of the following parameters:

- shelter thermostat setting

- various shelter electric loads

- configurable tiered load shedding in shelters

- microgrid modes (silent mode, individual generator control).

The SAGE energy management system was a 'proof of principle' that demonstrated an automated tool that energy managers or camp mayors could use to easily plan, monitor and manage operational energy. A full-fledged base camp energy management system could include deeper integration of meters and sensors, and also feature a broader and more comprehensive set of controls as have been implemented at several federal sites. A screen shot of the SAGE base camp energy management system dashboard is shown in Figure 2-8. 


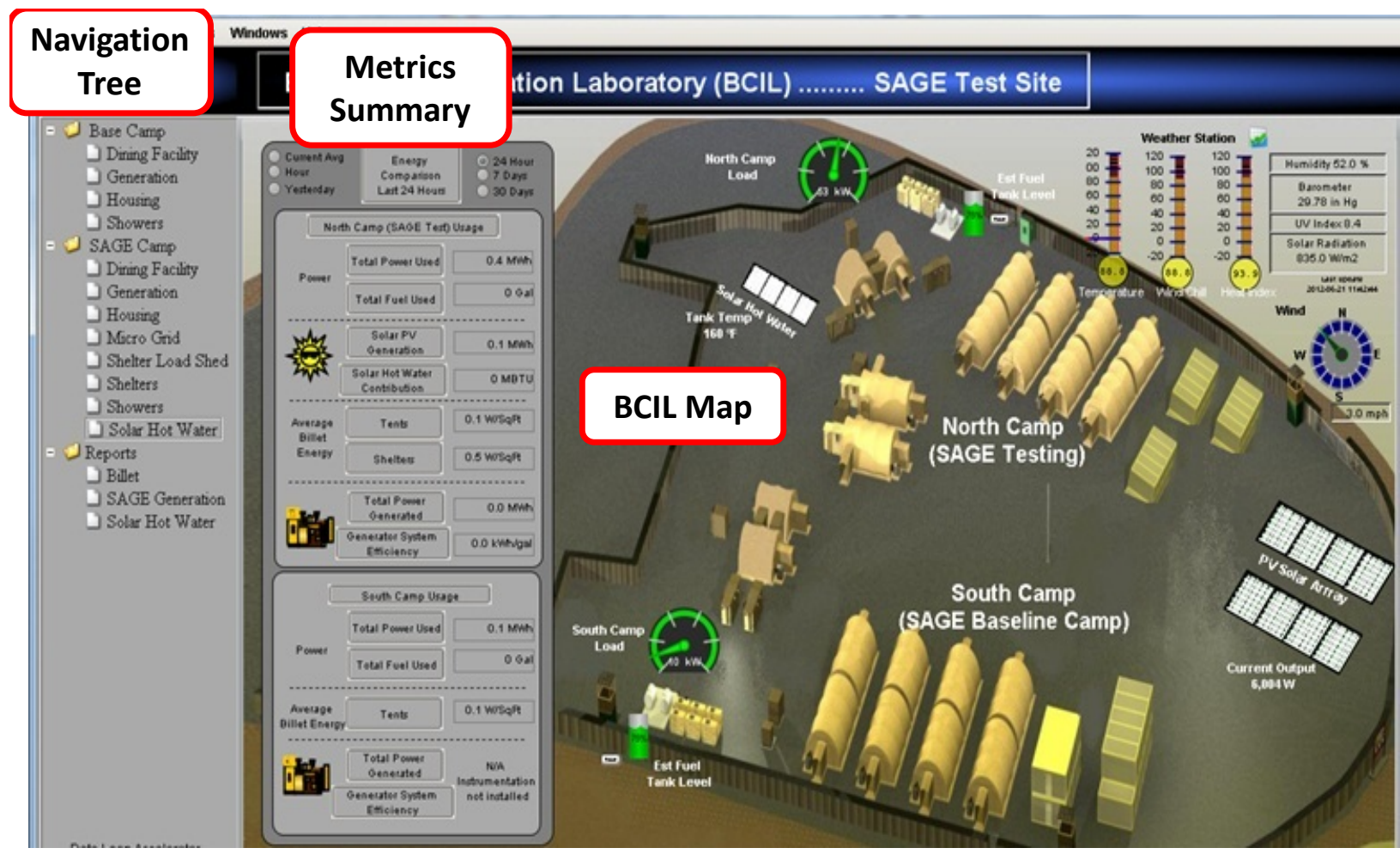

Figure 2-8. Base Camp Energy Management System

\subsection{Wood-Framed Buildings with Improvements Package}

The wood-framed building technology described in this section was not specifically tested in field prototypes at BCIL. Wood-framed buildings were modeled as replacements for all Baseline buildings in all base camp sizes globally. USACE informed LIA that in most contingency basing scenarios, engineers would rely on host nation support to purchase materials required for woodframing building technology vice purchasing, shipping and constructing energy efficiency shelters similar to those demonstrated during SAGE testing. The modeled buildings are stickframed, hard-walled buildings with wall, roof, and floor insulation (R-19). The improvements package includes high-efficiency heat pumps sized correctly for each building size and type and automated thermostat setbacks to $80^{\circ} \mathrm{F}$ and $68^{\circ} \mathrm{F}$ during unoccupied hours. This technology package is considered the best-case replacement of any existing Baseline building. For the 150Soldier base camps, both the SAGE energy efficient shelters and the wood-framed buildings with improvement package were modeled as separate scenarios. Specifically, the SAGE energyefficient-shelter scenario replaces the troop housing tents only. The wood-framed buildings with improvements package replaces every building in the 150-Soldier base camp. This modeling of two types of insulated buildings is unique to the 150-Soldier base camps; the modeling for the 600- and 5,000-Soldier base camps included only wood-framed buildings. 


\subsection{Testing Approach, Results, and Analysis}

\subsection{Testing Approach}

Testing occurred in three distinct phases from January 2012 to March 2013; it was designed to test SAGE prototype equipment in progressively more integrated environments in order to collect field data comparing SAGE against Baseline technology that could be used to build global SAGE models. With the progression of the three phases, test focus gradually shifted from demonstrating the technical capabilities of the SAGE equipment towards demonstrating the holistic energy savings of a SAGE-enabled base camp in one climate zone (East Coast U.S.). Test procedures were developed by PNNL personnel, who were also responsible for performing all tests in cooperation with Product Manager, Force Sustainment Systems (PM FSS) personnel, and witnessed by LIA representatives.

\subsubsection{Phase 1 - Microgrid and Storage Factory Tests}

Phase 1 tests focused only on the SAGE microgrid, the energy storage system, and the integration of a PV module. Testing demonstrated the viability and technical capabilities of the smart microgrid. The tests were specifically designed to evaluate the performance of the generators and the energy storage system in both an isolated setting and when integrated into a complete system. The tests initially explored normal operating conditions for all components and progressed to more rigorous tests (e.g., large and sudden load variations) that stressed the system to make sure it operated satisfactorily under extreme conditions.

Specific tests included verifying proper load sharing between generators and the energy storage system; integration of the PV array; stability of grid parameters (e.g., voltage and frequency); grid power quality when exposed to sudden large increases and decreases in load and generation capacity; and evaluating the supply duration of the energy storage system.

Testing was performed by Caterpillar personnel and witnessed by PNNL engineers. PNNL-designed test plans and procedures were used to direct and record the work. A load bank was used to simulate base camp loads on the microgrid.

\subsubsection{Phase 2 - Field Tests at BCIL with Load Bank}

Phase 2 tests focused on the SAGE microgrid, the energy storage system, the PV arrays, the energy efficient shelters, the SHW system, and the base camp energy management system. The primary objective was to individually test each piece of SAGE technology at the BCIL facility with simulated Soldier occupancy using a load bank. Direct comparisons between the SAGE base camp and the Baseline camp were, in principle, possible as the BCIL had two identical 150-Soldier base camps located side-by-side. One of these base camps was fully equipped with SAGE technology ('SAGE camp') while the remaining base camp represented baseline technology ('Baseline camp'). 
An electrical load study performed by TIAX for Force Provider in 2006 was scaled for testing purposes. This 'TIAX report' separated the electrical power profiles of a base camp into five sub-facilities (Soldier housing, latrines, laundry, kitchen, and showers). The microgrid, the energy storage system, and the PV system were tested against the combined TIAX load profile, i.e., all five sub-facilities simultaneously, via a load bank. To evaluate Baseline camp performance, the five individual sub-profiles were programmed into the load bank and tested against Baseline $60 \mathrm{~kW}$, TQG-60 generators. Testing of the microgrid occurred both with and without the PV arrays and allowed a comparison of fuel consumption between the SAGE microgrid and Baseline spot generation.

To test the performance of the energy efficient shelters against the Baseline air-beam tents, both building types were set to identical interior temperatures over several days in varying weather conditions and the energy consumption of these buildings was compared.

The SHW system was tested one day in the summer and one day in the fall using a PNNLdeveloped shower test procedure that replicated actual shower usage in a 150-Soldier base camp. The hot water demand for the test procedure was created by scaling off the consumption pattern of the shower TIAX profile. The developed demand was higher in the morning and evening than during the rest of the day, which is a common domestic hot water load pattern. ${ }^{6}$

The PNNL test procedure included a 15-hour period of manually turning showers on and off to simulate a shower demand, with preset targets for shower water temperatures and preset temperatures on the Army fuel-fired heaters. The test procedure was conducted during the same time period in both the SAGE camp and the Baseline camp. The Baseline and SAGE camps had identical shower facilities; however the hot water demand was supplied by an AWH 400 in the Baseline camp and by both the SHW system and an AWH 400 in the SAGE camp. A direct comparison between the camps was made.

Phase 2 data were used to create energy models for the calculation of annual performance of the SAGE and Baseline 150-Soldier base camps in 14 locations selected by LIA across seven global climate zones.

\subsubsection{Phase 3 - Field Tests at BCIL with Soldiers}

Soldier-occupancy testing occurred in October 2012 at the BCIL in Fort Devens, Massachusetts. The objective was to test all SAGE components together with Soldiers occupying both the SAGE and Baseline camps over a 72-hour test period. This final phase of testing provided a 'live' test with Soldier occupation to test and stress all SAGE components in an integrated setting. The microgrid with energy storage system and two PV arrays provided electric power to the SAGE camp, while six 60-kW TQG-60 spot generators provided electric power to the Baseline camp. The Baseline camp was occupied by one hundred (100) Reserve Officers' Training Corps cadets who used four of eight air-beam tents, the kitchen facility, latrines, and showers. The SAGE camp was occupied by one hundred fifty-seven (157) Military

\footnotetext{
${ }^{6}$ Mutch, JJ. 1974. Residential Water Heating: Fuel Conservation, Economics, and Public Policy. R-1498-NSF, RAND Corp., Santa Monica, CA.
} 
Police Reservists who used the three energy efficient shelters, all eight air-beam tents, the kitchen facility, the latrines and showers with SHW system, and laundry facility. In addition to this unequal number of occupants, the military police were at BCIL for a two-week-long training with regular tent use in the evenings, while the cadets used BCIL for only a three-day training that included substantial hours in field exercises, including nighttime exercises. This unequal occupancy resulted in unequal energy use that did not allow for a direct comparison of the two camps. Post-testing analysis equalized the occupancy and energy balance. The data collected from the military police occupancy was useful in modeling both Baseline and various SAGE technology base camps worldwide.

\subsubsection{Baseline and SAGE Camp Comparison}

When analyzing the energy savings gains from improved technology versus existing equipment, it is critical to establish a transparent, understandable, and sensible baseline. Baseline conditions serve as a reference point against which new technologies are compared and are the foundation of savings calculations. Furthermore, these savings calculations can be represented as energy savings such as kilowatt-hours $(\mathrm{kWh})$ or gallons of fuel saved or as relative savings such as percent reduction in energy consumption. For instance, if a Baseline camp consumes 100,000 gallons and a SAGE camp consumes 90,000 gallons, the SAGE camp has saved 10,000 gallons and is a 10\% improvement over the Baseline. If instead a Baseline is selected that consumes 500,000 gallons and compared against the same SAGE camp, the savings figure grows to 410,000 gallons, which represents savings of over $80 \%$ despite the fact that the SAGE camp consumption has remained static at 90,000 gallons. This demonstrates the criticality of selecting a sensible and fair baseline against which to evaluate new technologies.

This report documents savings both in terms of energy saved (e.g., kWh or gallons saved) as well as percent savings (i.e., 10\% savings over the Baseline). To holistically and transparently understand these savings, it is important to understand the Baseline technologies and how they contrast with SAGE technologies. For SAGE tests, the Baseline camp was a preconfigured 150Soldier Force Provider base camp. During SAGE's tenure at BCIL, PM FSS made many energy related improvements to the 150 -Soldier base camp, including improved power generation and insulated tent liners. To maintain consistency throughout all of SAGE's test phases, the SAGE team evaluated the technologies against the original BCIL base camp configuration. Table 3-1 provides a technology comparison between the Baseline and SAGE camps, as implemented at the BCIL at Fort Devens during field testing of SAGE technology prototypes, that would serve to create, calibrate, and validate computer models of annual base camp models of all sizes. 
Table 3-1. BCIL Camp and SAGE Camp Comparison

\begin{tabular}{|c|c|c|}
\hline Technology & Baseline & SAGE Counterpart \\
\hline Power Generation & $\begin{array}{l}\text { Spot generation consisting of six } \\
60 \mathrm{~kW} \text { tactical quiet generators } \\
\text { (TQG-60) supplying six separate } \\
\text { load groups }\end{array}$ & $\begin{array}{l}\text { Smart microgrid consisting of three } \\
\text { commercial } 100 \mathrm{~kW} \text { generators and } \\
\text { optional retrofitted } 60 \mathrm{~kW} \text { TQG that } \\
\text { powers the entire base camp via a grid }\end{array}$ \\
\hline Energy Storage & No energy storage system & $\begin{array}{l}\text { Energy storage system with bidirectional } \\
\text { inverter. }\end{array}$ \\
\hline Renewables & No renewables & $12.9 \mathrm{~kW}$ PV array \\
\hline Tents & $\begin{array}{l}\text { Uninsulated air-beam tents with } \\
\text { standard lights, } 25 \mathrm{~kW} \mathrm{ECU}^{(\mathrm{a})}\end{array}$ & $\begin{array}{l}\text { Insulated modular, hard-walled shelter } \\
\text { with LED lights, high-efficiency } 3 \mathrm{~kW} \\
\text { heat pump }\end{array}$ \\
\hline Hot Water Heating & AWH 400 fuel-fired water heater & $\begin{array}{l}\text { SHW system supplementing an } \\
\text { AWH } 400 \text { fuel-fired water heater for } \\
\text { showers only }\end{array}$ \\
\hline $\begin{array}{l}\text { Base Camp Energy } \\
\text { Management System }\end{array}$ & $\begin{array}{l}\text { No base camp energy management } \\
\text { system }\end{array}$ & $\begin{array}{l}\text { DSOM software configured for SAGE } \\
\text { and BCIL and installed on a dedicated } \\
\text { SAGE PC at BCIL }\end{array}$ \\
\hline
\end{tabular}

Similarly, Table 3-2 provides a technology comparison between the Baseline and improved technologies at the 150-Soldier, 600-Soldier, and 5,000-Soldier base camps that were fully modeled for an entire year in fourteen different global locations. The 150-Soldier and 600Soldier base camps are grouped together, as the 600-Soldier base camp is, generally speaking, a fourfold scale-up of the 150-Soldier base camp plus some additional buildings. Camps of both sizes contain much of the same Baseline technology.

The 5,000-Soldier Baseline camp has technology in line with the base camp's more permanent nature. These large base camps are typically hard-walled buildings with insulation, and use HVAC units that are more energy efficient than those of smaller base camps. Water heating is electric. The power generation consists of a spot generation system with nine separate $3,000 \mathrm{~kW}$ generators. An underloaded spot generation arrangement is highly inefficient. While this $9 \times 3000 \mathrm{~kW}$ spot generation scheme originated in USACE specifications for 5,000-Soldier base camps, it is not clear if it has been fully implemented at an existing base camp. Since the fuel savings numbers for a microgrid in a 5,000-Soldier base camp found in this report use the USACE specifications as the Baseline, it should be considered that a base camp with less efficient systems could see higher fuel savings. 
Table 3-2. Baseline Camp and SAGE Camp Comparison for all Modeled Camps Worldwide

\begin{tabular}{|c|c|c|}
\hline Technology & Baseline & SAGE Counterpart \\
\hline \multicolumn{3}{|c|}{ 150-Soldier and 600-Soldier Base Camps } \\
\hline Power Generation & $\begin{array}{l}\text { 150-Soldier: } 6 \times 60 \mathrm{~kW} \text { TQGs spot } \\
\text { generation } \\
\text { 600-Soldier: } 24 \times 60 \mathrm{~kW} \text { TQGs spot } \\
\text { generation }\end{array}$ & $\begin{array}{l}\text { 150-Soldier: Smart microgrid of } 3 \times 100 \\
\text { kW commercial generators } \\
600 \text {-Soldier: Smart microgrid of } 4 \times 300 \\
\text { kW commercial generators }\end{array}$ \\
\hline Energy Storage & No energy storage system & $\begin{array}{l}\text { 150-Soldier: } 250 \mathrm{kWh} \text { energy storage } \\
\text { system with bidirectional inverter } \\
600 \text {-Soldier: No energy storage system }\end{array}$ \\
\hline Renewables & No renewables & $\begin{array}{l}\text { 150-Soldier: } 51.6 \mathrm{~kW} \mathrm{PV} \\
\text { 600-Soldier: } 206.4 \mathrm{~kW} \mathrm{PV} \\
\text { PV sized to meet tent loads. }\end{array}$ \\
\hline Tents & $\begin{array}{l}\text { Uninsulated air-beam tents with } \\
\text { standard fluorescent lighting, and } \\
25 \mathrm{~kW} \mathrm{ECU}\end{array}$ & $\begin{array}{l}\text { 1. Insulated modular, hard-walled } \\
\text { shelter with LED lights, high- } \\
\text { efficiency } 3 \mathrm{~kW} \text { heat } \\
\text { 2. Wood-framed, insulated (R-19) } \\
\text { buildings with high-efficiency heat } \\
\text { pumps and automated thermostat } \\
\text { setbacks }\end{array}$ \\
\hline $\begin{array}{l}\text { All Other Camp } \\
\text { Buildings }\end{array}$ & $\begin{array}{l}\text { Uninsulated air-beam tents with } \\
\text { standard lighting, oversized ECUs } \\
\text { and Tricon containers }\end{array}$ & $\begin{array}{l}\text { Wood-framed, insulated (R-19) buildings } \\
\text { with high-efficiency heat pumps and } \\
\text { automated thermostat setbacks }\end{array}$ \\
\hline Hot Water Heating & AWH 400 fuel-fired water heater & $\begin{array}{l}\text { SHW system supplementing an } \\
\text { AWH } 400 \text { fuel-fired water heater for } \\
\text { only showers in the } 150 \text {-Soldier base } \\
\text { camp, and for all hot water in the } \\
600 \text {-Soldier base camp }\end{array}$ \\
\hline \multicolumn{3}{|c|}{ 5,000-Soldier Base Camp } \\
\hline Power Generation & $\begin{array}{l}9 \times 3,000 \mathrm{~kW} \text { commercial generators } \\
\text { (spot generation) }\end{array}$ & $\begin{array}{l}\text { Microgrid of } 4 \times 3,000 \mathrm{~kW} \text { commercial } \\
\text { generators }\end{array}$ \\
\hline $\begin{array}{l}\text { All Base camp } \\
\text { buildings }\end{array}$ & $\begin{array}{l}\text { Generally wood-frame, insulated } \\
\text { buildings }\end{array}$ & $\begin{array}{l}\text { Wood-framed, insulated (R-19) buildings } \\
\text { with right-sized high-efficiency heat } \\
\text { pumps and automated thermostat } \\
\text { setbacks }\end{array}$ \\
\hline Hot Water Heating & Standard electric water heating & $\begin{array}{l}\text { Solar hot water heating supplementing } \\
\text { standard electric water heaters for all } \\
\text { base camp hot water }\end{array}$ \\
\hline
\end{tabular}

\subsection{Test Results and Analysis}

\subsubsection{Phase 1 - Results of Microgrid and Storage Factory Tests}

All Phase 1 tests (microgrid and battery) were executed successfully and the system was accepted for further testing at BCIL. The microgrid demonstrated its ability to properly share loads between individual generators and the energy storage system. This load sharing capability 
is the key feature that enabled the system to perform at better fuel economy than comparable spot generation setups. The microgrid system was shown to properly integrate the chosen SAGE PV technology and manage electrical load transients (the sudden enabling or disabling of large electrical loads) with and without the energy storage system. During this testing, the energy storage system proved to provide better grid frequency and voltage stability (i.e., power quality) to the microgrid as a whole, thereby improving the reliability and electric quality of the microgrid system. The "silent run" mode- the ability of the microgrid's energy storage system, without any conventional generators, to power a reduced base camp load of $100 \mathrm{~kW}$-was demonstrated successfully. Fuel consumption under the 24-hour TIAX load profile was established at 324 gallons of diesel, but the test did not include a spot generation system to use as a baseline.

\subsubsection{Phase 2 - Results of Field Tests at BCIL with Load Bank}

Phase 2 testing at the BCIL at Fort Devens conclusively demonstrated that SAGE technology could save significant amounts of fuel or energy compared to the Baseline counterparts.

Table 3-3 presents Phase 2 energy savings results for each SAGE technology tested over a 24-hour period. Energy savings are presented via several different metrics. First, the total fuel use requirements in gallons are documented, followed by fuel savings in gallons and percent reduction. The last column compares the various base camp configurations in terms of gallons of source fuel used per Soldier per day (based on 150 Soldiers), allowing fast and meaningful comparisons. Data for the base camp energy management system was not included in this table. The impact of this system was modeled for annualization purposes only.

The first four rows compare a Baseline camp against the same base camp with only a microgrid and energy storage system, a base camp with only PV arrays, and a base camp with all three technologies. The electricity provided by the PV was set to match the quantity of eight tents: $6.46 \mathrm{~kW}$ per field-tested BCIL array multiplied by eight tents for a base camp-wide total of $52 \mathrm{~kW}$.

The replacement of spot generation in a 150-Soldier base camp with a SAGE microgrid and energy storage system produced a $20.6 \%$ reduction in overall fuel consumption. The addition of $52 \mathrm{~kW}$ PV arrays to the Baseline camp resulted in a $12.3 \%$ fuel reduction on a day with little cloud cover. The fourth row shows that a combination of a $52 \mathrm{~kW}$ PV system with a microgrid and energy storage system resulted in a $30.1 \%$ overall reduction in fuel use. The percent savings per Soldier per day closely follows the overall base camp savings percentages.

Table 3-3 directly compares Baseline air-beam tents against the SAGE energy efficient shelters with high-efficiency heat pumps, resulting in the most significant reduction of fuel use for any single SAGE technology. Fuel used for eight energy efficient shelters, as well as source fuel per Soldier per day, is $83.0 \%$ less than those of eight air-beam tents. 
Table 3-3. Phase 2 Fuel Savings by Technology

\begin{tabular}{|c|c|c|c|c|}
\hline & $\begin{array}{l}\text { Total Source } \\
\text { Fuel Used } \\
\quad \text { (gal) }\end{array}$ & $\begin{array}{l}\text { Source Fuel } \\
\text { Saved (gal) }\end{array}$ & $\begin{array}{c}\text { Percent } \\
\text { Source Fuel } \\
\text { Saved }\end{array}$ & $\begin{array}{l}\text { Source Fuel Used per } \\
\text { Soldier per Day } \\
\text { (gal/Soldier/day) }\end{array}$ \\
\hline \multicolumn{5}{|c|}{ Baseline vs. SAGE Camp Energy Savings } \\
\hline Baseline Camp & 346.4 & $\mathrm{n} / \mathrm{a}$ & $\mathrm{n} / \mathrm{a}$ & 2.31 \\
\hline $\begin{array}{l}\text { Baseline Camp + Microgrid with } \\
\text { Energy Storage System }\end{array}$ & 275.0 & 71.4 & $20.6 \%$ & 1.83 \\
\hline Baseline Camp + $52 \mathrm{~kW}$ PV Array & 303.7 & 42.7 & $12.3 \%$ & 2.02 \\
\hline $\begin{array}{l}\text { Baseline Camp + Microgrid with } \\
\text { Energy Storage System }+52 \mathrm{~kW} \\
\text { PV Array }\end{array}$ & 242.3 & 104.1 & $30.1 \%$ & 1.62 \\
\hline \multicolumn{5}{|c|}{ Baseline Tent vs. Energy Efficient Shelter } \\
\hline Air-Beam Tents & 54.1 & $\mathrm{n} / \mathrm{a}$ & $\mathrm{n} / \mathrm{a}$ & 0.36 \\
\hline Energy Efficient Shelters & 9.2 & 44.9 & $83.0 \%$ & 0.06 \\
\hline \multicolumn{5}{|c|}{ Baseline Shower Water Heating vs. Solar Hot Water } \\
\hline Baseline Shower Water Only & 9.6 & $\mathrm{n} / \mathrm{a}$ & $\mathrm{n} / \mathrm{a}$ & .06 \\
\hline Baseline Heater + SHW System & 2.75 & 6.9 & $71 \%$ & .02 \\
\hline
\end{tabular}

Table 3-3 contrasts the fuel use of the Baseline standard Army fuel-fired hot water heater against the combined SAGE SHW system and Army fuel-fired heater. The SAGE SHW system supplies heated water to the standard Army fuel-fired water heater to meet a target temperature; thus, if the water supplied to the fuel-fired heater is hot enough, the heater never turns on. This was the case for the majority of the day during the first test performed in June and also for many hours during the second test performed in October. This reduction of the operating hours for the standard Army fuel-fired water heater working in combination with the SHW system resulted in an average $71 \%$ fuel savings. Although the SAGE Phase 2 tests were limited to two days, other researchers performing long-term testing with similar flat-plate collector solar systems and supplemental heaters have also observed significant savings (58\% over a standard electric water heater ${ }^{7}$ ) associated with underutilization of auxiliary heaters. ${ }^{8}$ The data in Table 3-3 demonstrate that the best performing technologies in terms of percent of fuel saved are the energy efficient shelters, the SHW system, and the microgrid, respectively. A combination of these technologies could result in a significant amount of fuel saved.

\footnotetext{
${ }^{7}$ Colon, CJ and DS Parker. 2013. Side-by-Side Testing of Water Heating Systems: Results from 2010-2011

Evaluation. Florida Solar Energy Center/University of Central Florida. March 2013. Accessed January 17, 2014 at www.fsec.ucf.edu/en/publications/pdf/FSEC-RR-386-12.pdf.

${ }^{8}$ Colon, CJ and DS Parker. 2010. Side-by-Side Testing of Water heating Systems: Results from 2009-2010 Evaluation. FSEC-CR-1856-10, Florida Solar Energy Center/University of Central Florida. June 2010. Accessed January 17, 2014 at http://www.fsec.ucf.edu/en/publications/pdf/FSEC-CR-1856-10.pdf.
} 


\subsubsection{Phase 3 - Results of Field Tests at BCIL with Soldiers}

The test approaches for Phases 2 and 3 shared many common features. Both tests attempted to evaluate the performance of the entire Baseline and the complete SAGE camp with all SAGE equipment. In Phase 2, a known and well-understood load profile (TIAX) was used to test the SAGE equipment with a load bank. However, in Phase 3, the Soldiers occupying the base camps imposed their own loads based on training activity and operations tempo. Consequently, the loads imposed on the base camps differed between Phases 2 and 3. Because equipment performance was closely related to the imposed loads, comparisons in the performance of Baseline and SAGE camps between Phases 2 and 3 were addressed carefully. Phase 3 tests permitted the collection of actual Soldier load data. To facilitate cross-phase comparisons, it is beneficial to review the load profiles for the Phase 2 and 3 tests as follows.

Figure 3-1 plots the TIAX and Soldier-imposed loads over a 24-hour period. As can be seen, the TIAX load profile averaged higher loads than the actual Soldier-imposed loads, which will affect equipment performance and energy savings calculations. However, it should be emphasized that merely because Phase 3 testing observed significantly different loads between the TIAX report and the Soldier occupancy, these results do not conclusively resolve how the observed loads vary from loads in new base camp designs or in existing as-built base camps. Loads are contingent on season, climate, mission, operations tempo, and a host of additional considerations. This observation should be accounted for when comparing results across the SAGE test phases.

\section{Hour TIAX Profile versus 24 Hour Soldier Profile}

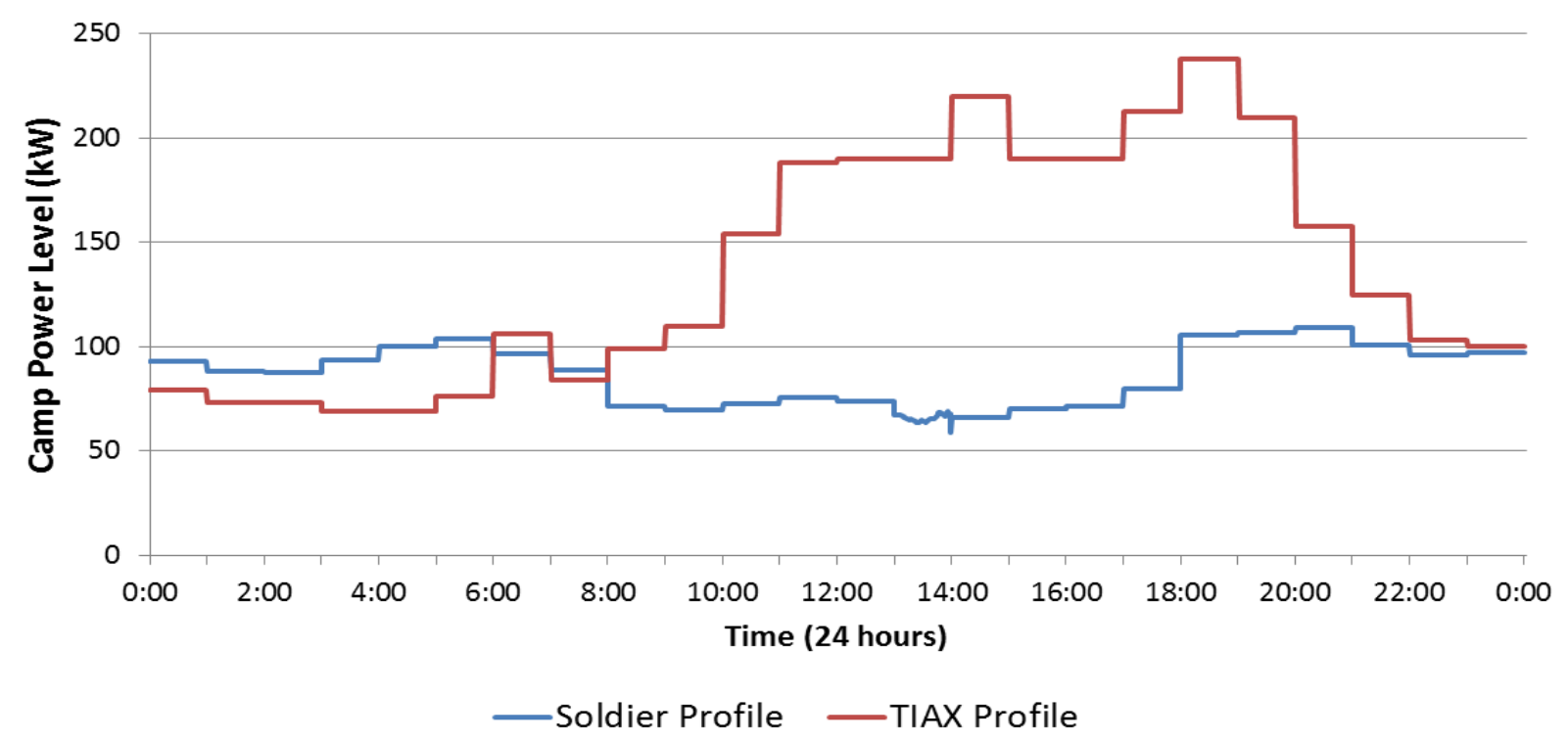

Figure 3-1. 24-Hour TIAX Profile vs. 24-Hour Soldier Profile

The significance of the load profiles shown in Figure 3-1 lies in the resulting fuel efficiency found in the spot generators. The higher power levels in the TIAX profiles (Phase 2) loaded the spot generators more than the Soldier-imposed profiles (Phase 3), resulting in higher 
spot-generation efficiency during Phase 2 testing compared to Phase 3 . The microgrid's fuel efficiency was identical in both phases but the microgrid showed higher percentage improvement over the Baseline in Phase 3 because the smaller Phase 3 load profile loaded the spot generators to lower levels that significantly lowered their fuel efficiency.

\subsubsection{Solar Hot Water Analysis}

The purpose of the SHW tests was to demonstrate the scale of fuel savings provided by a SHW heating system in the holistic context of a base camp. The SHW system was tested in two phases; Phase 2 simulation tests were performed in June and October 2012, and Phase 3 occupancy tests were performed in October 2012.

The analysis of the field tests involved an energy-balance (inputs and outputs) estimate over time for the defined control volumes, including the SHW system and the AWH 400 heaters. To accurately annualize and globalize the test results was more a difficult estimate, and thus, a few approaches were taken, including solar fractions (as defined by ASHRAE 95-1987 ${ }^{9}$ ); system efficiencies were derived. For global savings estimates, however, the modeling provided the annual energy and cost savings.

\subsubsection{Phase 3 Energy Savings by Technology}

The results of Phase 3 testing confirmed and validated the results obtained in Phase 2 testing. The technologies with the largest fuel or energy savings impact were the energy efficient shelters and microgrid with energy storage system.

Table 3-4 presents Phase 3 energy savings results for each SAGE technology tested over a 24-hour period. Energy savings are presented via several different metrics, as was done in Table 3-3, Phase 2 savings. First, the total fuel use requirements in gallons are documented, followed by fuel savings in gallons and percent reduction. The last column compares the various base camp configurations in terms of gallons of source fuel used per Soldier per day, allowing fast and meaningful comparisons.

The first four rows contrast the Baseline camp against the same base camp with a microgrid and energy storage system, a base camp with only the PV array, and a base camp equipped with microgrid, energy storage system, and PV. The replacement of spot generation in the 157-Soldier base camp with a SAGE microgrid and energy storage system resulted in a $33.7 \%$ reduction in fuel consumption and $34.0 \%$ reduction in source fuel per Soldier per day.

The addition of $52 \mathrm{~kW}^{10} \mathrm{PV}$ arrays to the Baseline camp (no microgrid or storage) resulted in only a $2.1 \%$ fuel savings over the Baseline, compared to the $12.3 \%$ reduction observed in Phase 2 for the identical case. There are multiple explanations for this difference. First, weather data showed that the Phase 3 October environmental conditions provided 55.3\% less insolation than

\footnotetext{
${ }^{9}$ Methods of testing to determine the thermal performance of solar domestic water heating systems (ASHRAE Standard 95-198 7).

${ }^{10}$ As done for the Phase 2 calculations (Table 3-3), the Phase 3 calculations also used the field-measured PV output of the two installed arrays and multiplied the output to represent a total of eight arrays, one for each of eight tents.
} 
during Phase 2. Second, the Phase 3 actual load profiles were smaller compared to the TIAX profiles used in Phase 2. Third, the $52 \mathrm{~kW}$ PV array power was assumed to be split evenly between the two $60 \mathrm{~kW}$ spot generators serving the eight Soldier tents because no microgrid existed in this scenario. The PV-provided power resulted in a lower load on those generators, and therefore lower efficiency than before. In many periods, the PV power met the entire load and the generators would idle at zero load, thereby still burning fuel while not supplying any energy at all.

Table 3-4. Phase 3 Energy Savings by Technology

\begin{tabular}{|c|c|c|c|c|}
\hline & $\begin{array}{c}\text { Total } \\
\text { Source Fuel } \\
\text { Used (gal) }\end{array}$ & $\begin{array}{l}\text { Source Fuel } \\
\text { Saved (gal) }\end{array}$ & $\begin{array}{c}\text { Percent } \\
\text { Source } \\
\text { Fuel Saved }\end{array}$ & $\begin{array}{c}\text { Source Fuel per Soldier } \\
\text { per Day } \\
\text { (gal/Soldier/day) }\end{array}$ \\
\hline \multicolumn{5}{|c|}{ Baseline vs. SAGE Camp Energy Savings } \\
\hline Baseline Camp & 284.7 & $\mathrm{n} / \mathrm{a}$ & $\mathrm{n} / \mathrm{a}$ & 1.81 \\
\hline $\begin{array}{l}\text { Baseline Camp + Microgrid } \\
\text { with Energy Storage }\end{array}$ & 186.7 & 95.0 & $33.7 \%$ & 1.19 \\
\hline $\begin{array}{l}\text { Baseline Camp + } 52 \mathrm{~kW} \text { PV } \\
\text { Array }\end{array}$ & 275.8 & 5.8 & $2.1 \%$ & 1.76 \\
\hline $\begin{array}{l}\text { Baseline Camp + Microgrid } \\
\text { with Energy Storage }+52 \mathrm{~kW} \\
\text { PV Array }\end{array}$ & 172.2 & 109.5 & $38.5 \%$ & 1.10 \\
\hline \multicolumn{5}{|c|}{ Baseline Tent vs. Energy Efficient Shelter } \\
\hline Air-Beam Tents & 109.7 & $\mathrm{n} / \mathrm{a}$ & $\mathrm{n} / \mathrm{a}$ & 0.73 \\
\hline Energy Efficient Shelters & 33.4 & 76.33 & $69.6 \%$ & 0.22 \\
\hline \multicolumn{5}{|c|}{ Baseline Shower Water Heating vs. Solar Hot Water } \\
\hline & $\begin{array}{c}\text { Source } \\
\text { Fuel/Gal } \\
\text { Water Used } \\
\text { (gal fuel/gal } \\
\text { water) }\end{array}$ & $\begin{array}{l}\text { Source Fuel } \\
\text { Saved/Gal } \\
\text { Water (gal } \\
\text { fuel/gal water) }\end{array}$ & $\begin{array}{c}\text { Source } \\
\text { Fuel } \\
\text { Saved }(\%) \\
\end{array}$ & $\begin{array}{l}\text { Source Fuel/Soldier/Day } \\
\text { (gal fuel/gal } \\
\text { water/Soldier/day)*1000 }\end{array}$ \\
\hline Baseline Shower Water Only & .009 & $\mathrm{n} / \mathrm{a}$ & $\mathrm{n} / \mathrm{a}$ & .059 \\
\hline $\begin{array}{l}\text { Baseline Heater + SHW } \\
\text { System }\end{array}$ & .005 & .004 & $42 \%$ & .035 \\
\hline
\end{tabular}

The fourth row illustrates that a combination of the SAGE microgrid plus energy storage system and the $52 \mathrm{~kW}$ PV system results in a $38.5 \%$ fuel savings and $39.2 \%$ savings in source fuel per Soldier per day over the Baseline camp.

Table 3-4 directly compares Baseline air-beam tents against the SAGE energy efficient shelters with high-efficiency heat pumps, resulting in the most significant reduction of fuel use for any single SAGE technology. Fuel used for eight energy efficient shelters, as well as source fuel per Soldier per day, is $69.6 \%$ less than those of eight air-beam tents. 
Similar to the Phase 2 results, the last two rows in the table compare the Baseline standard Army fuel-fired hot water heater against the combined SAGE SHW system/Army fuel-fired heater. However, the 157 SAGE base camp occupants (military police) used the SAGE camp showers and the 100 Baseline camp occupants used the Baseline camp showers with highly variable consumption rates (approximately 1930 gallons used in the SAGE camp showers and 505 gallons used in the Baseline camp showers). Therefore the data was normalized to make the comparison. The quantity 'gallons of fuel consumption per gallon of water consumed' was the unit of measure used for the comparison. The results indicated that despite one of the three days being mostly cloudy, there was a $42 \%$ fuel savings per gallon of water used between the two base camps.

\subsection{Annualization and Globalization}

The results of Phases 1 (microgrid tests at factory), 2 (load-bank tests at BCIL), and 3 (occupied period at BCIL) were used to construct and calibrate models of the Baseline and SAGE buildings to credibly estimate annual energy and fuel numbers in fourteen different global locations for three different base camp sizes (150-, 600-, and 5,000-Soldier base camps). The specific makeup of each base camp size is provided in Appendix B. The modeling methodology and assumptions are described in Appendix D.

The power profiles gained from the Phase 2 and Phase 3 tests were used for computerized models of buildings used in the 150- and 600-Soldier base camps. The buildings found in the larger 5,000-Soldier base camps were modeled with commercially available buildingcharacteristics data for buildings and building equipment. The models were calibrated against test data from Phases 2 and 3 for the Fort Devens location. Lastly, the models were combined with commercially available climate data to get fuel and energy numbers for the remaining thirteen global locations.

The power and energy profiles for all buildings were created with the DOE-developed EnergyPlus software and simulated in hourly increments for one entire year. Simulated buildings included the following:

- Baseline tents and containerized structures found in both a standard 150-Soldier Force Provider module managed by PM FSS and in standard AFCS/TCMS drawings maintained by USACE

- Baseline buildings according to the USACE standards for 600-Soldier and 5,000-Soldier base camps

- improved buildings (generally hard-walled, insulated buildings)

- various iterations of improved buildings, including high-efficiency heat pumps, SHW heating, better insulation, thermostat setbacks, and a combined package of all improvements.

EnergyPlus models energy and water use in buildings and is capable of simultaneously accounting for building elements such heating, cooling, lighting, ventilation, plug loads, and other internal gains when performing energy simulations. When used properly, building-energy 
models such as EnergyPlus models are capable of more accurately predicting building energy consumption across a variety of climate zones than spreadsheet tools or coarse extrapolative/correlative approaches. Incorporated in the EnergyPlus model for the energy efficient shelters was a setback in thermostat temperature and de-energizing of some shelter convenience outlets during nighttime hours. This capability is inherent to the base camp energy management software (DSOM) when coupled with remotely controllable electric service panels for the shelters.

Energy output of a PV system was also simulated on an hourly basis for all locations. USACE's camp standards were used to assemble virtual 150-, 600-, and 5,000-Soldier base camps in many different combinations of technology that were then simulated to run on multiple electric power generation options for an entire year for each location. Custom programming and scripting was used for this process.

The efficiency of each spot generation system and microgrid-except for the prototype microgrid at Fort Devens-was calculated for each hour of the year based on published efficiency data from the generator vendors. The efficiency of the prototype microgrid at Fort Devens was determined experimentally and did not vary significantly with load. It was created as a constant-efficiency microgrid for 150-Soldier base camp calculations only.

\subsubsection{Annual Savings by Technology at BCIL}

This section presents the results of the SAGE modeling analysis by technology, for all three base camp sizes in the Fort Devens location only. Results in other locations are similar but vary with climate.

Table 3-5 presents annual fuel savings as percentages for the 150-Soldier base camp at Fort Devens in a technology combination matrix that allows plug-and-play combination of different SAGE technologies. The savings values are presented as source-energy percentage savings, which in this case is equivalent to percentage of fuel saved. ${ }^{11}$

To use the matrix in Table 3-5 to estimate energy savings, identify a technology package along one axis and complement it to another technology package along the other axis. For instance, to estimate the savings of a microgrid and a PV array over a Baseline camp, first find the Baseline along the left hand column (located one row down from the reference cell) and then find microgrid + PV array along the top row (located three columns to the right). This energy savings is $46.8 \%$ over the Baseline. Similarly, the energy savings of a Baseline camp with energy efficient shelters (located four rows down from the reference cell) plus a microgrid (located two columns to the right) is $64.0 \%$.

\footnotetext{
${ }^{11}$ Source energy represents the energy consumed by a power plant to produce useful energy. Source energy can be measured in terms of gallons of diesel, tons of coal, cubic feet of natural gas, etc. Conversely, site energy represents energy consumed at the final point of consumption. Site energy includes the electricity used by homes to power lights or the natural gas used to heat water. It is useful to distinguish between source and site energy. For instance, saving $1 \mathrm{kWh}$ at home saves more than $1 \mathrm{kWh}$ of fuel at the power plant due to power plant and electricity distribution inefficiencies. Consequently, energy analysts often distinguish between source and site energy to help an audience understand the precise nature of energy consumption or savings.
} 
Table 3-5. 150-Soldier Camp Annual Source Energy Savings by Technology (Fort Devens)

\begin{tabular}{lccccc}
\hline & & Add & $\begin{array}{c}\text { Add } \\
\text { Microgrid, PV } \\
\text { Arrays }\end{array}$ & $\begin{array}{c}\text { Add Microgrid, } \\
\text { PV Arrays, } \\
\text { Shelters }\end{array}$ & $\begin{array}{c}\text { Add Microgrid, } \\
\text { PV, Shelters, } \\
\text { SHW }\end{array}$ \\
\hline Baseline & $0 \%$ & $35.8 \%$ & $46.8 \%$ & $65.3 \%$ & $66.9 \%$ \\
Add only microgrid & $35.8 \%$ & $35.8 \%$ & $46.8 \%$ & $65.3 \%$ & $66.9 \%$ \\
Add only PV arrays & $2.5 \%$ & $46.8 \%$ & $46.8 \%$ & $65.3 \%$ & $66.9 \%$ \\
$\begin{array}{l}\text { Add only energy } \\
\text { efficient shelters }\end{array}$ & $17.4 \%$ & $64.0 \%$ & $65.3 \%$ & $65.3 \%$ & $66.9 \%$ \\
$\begin{array}{l}\text { Add only SHW } \\
\begin{array}{l}\text { Add only wood- } \\
\text { framed buildings } \\
\text { package }\end{array}\end{array}$ & $1.0 \%$ & $36.8 \%$ & $66.3 \%$ & $66.3 \%$ & $66.3 \%$ \\
\hline
\end{tabular}

Replacing spot generation with a microgrid and energy storage system could yield the largest savings for any single technology package (35.8\%). The largest combined fuel savings is $80.8 \%$ for a base camp with all technology upgrades, including wood-framed buildings throughout the base camp. However, even just a two-technology combination of microgrid and energy efficient shelters (to replace eight troop housing tents) results in $64.0 \%$ annual source energy (fuel) saved. Replacing all base camp structures with wood-framed buildings and the matching improvement package (high-efficiency heat pumps, thermostat setbacks) and replacing the spot generation with a microgrid could result in $74.2 \%$ fuel savings annually. Fuel savings numbers were not calculated for the case of upgrading all base camp structures to wood-framed buildings only (without a change to a microgrid). The savings for this single scenario were only estimated as it is not considered to be a credible scenario due to severe inefficiencies in the Baseline spot generation system. If such a scenario were desired, at a minimum the spot generation would need to be downsized to match the reduced electrical loads resulting from the structure replacements.

Similarly, Table 3-6 shows the annual fuel savings in percent for a 600-Soldier base camp at Fort Devens in an identical technology combination matrix. The 600-Soldier base camp is essentially a fourfold scaled-up version of the 150-Soldier base camp, but does include some additional structure types not found in the smaller base camp.

The single biggest fuel-saving technology in this case is the replacement of all Baseline structures with wood-framed, insulated buildings with high-efficiency heat pumps and automated thermostat setbacks (the improvement package). This item alone saves $40.2 \%$ fuel annually in the Fort Devens location. Combining the wood-framed buildings with a microgrid to replace the Baseline spot generation saves $\mathbf{7 9 . 7 \%}$ fuel annually, making this two-technology combination particularly effective - analogous to the 150-Soldier base camp. The best case, a combination of all technologies, results in $83.7 \%$ fuel savings. 
Table 3-6. 600-Soldier Camp Annual Source Energy Savings by Technology (Fort Devens)

\begin{tabular}{|c|c|c|c|c|}
\hline & Baseline & $\begin{array}{c}\text { Add } \\
\text { Microgrid } \\
\end{array}$ & $\begin{array}{c}\text { Add } \\
\text { Microgrid, PV } \\
\text { Arrays } \\
\end{array}$ & $\begin{array}{c}\text { Add Microgrid, PV, } \\
\text { Wood-Framed Buildings }\end{array}$ \\
\hline Baseline & $0 \%$ & $29.6 \%$ & $33.4 \%$ & $83.5 \%$ \\
\hline Add only microgrid & $29.6 \%$ & $29.6 \%$ & $33.4 \%$ & $83.5 \%$ \\
\hline Add only PV arrays & $3.2 \%$ & $33.4 \%$ & $33.4 \%$ & $83.5 \%$ \\
\hline $\begin{array}{l}\text { Add only wood-framed } \\
\text { buildings package }\end{array}$ & $40.2 \%$ & $79.7 \%$ & $83.5 \%$ & $83.5 \%$ \\
\hline Add only SHW & $0.2 \%$ & $29.9 \%$ & $33.6 \%$ & $83.7 \%$ \\
\hline
\end{tabular}

Table 3-7 shows the annual fuel savings for a 5,000-Soldier base camp at Fort Devens. The single largest fuel-saving improvement of $43.4 \%$ comes from replacing the Baseline spot generation system with a microgrid. The identified Baseline spot generation system of nine 3 MW generators is inefficient and a microgrid brings fuel consumption to a more normal level. Similar savings could likely be obtained by reducing the number of spot generators to fit the base camp load more closely.

All other technologies in Table 3-7 show rather modest fuel savings of $0.1 \%$ to $3.0 \%$. The reason for this modest savings is that the existing Baseline 5,000-Soldier wood-frame base camp design standard has good, energy efficient buildings. Improvements can certainly be realized with better wood-framed buildings and improvement packages and a SHW system, but they are far less significant savings in the 5,000-Soldier base camp than in smaller base camps with their characteristically more temporary and less efficient tents and containers.

Table 3-7. 5,000-Soldier Camp Annual Source Energy Savings by Technology (Fort Devens)

\begin{tabular}{lcccc}
\hline & Baseline & Add Microgrid & $\begin{array}{c}\text { Add Microgrid, } \\
\text { PV Arrays }\end{array}$ & $\begin{array}{c}\text { Add Microgrid, PV, } \\
\text { Wood-Framed Buildings }\end{array}$ \\
\hline Baseline & $0 \%$ & $43.4 \%$ & $46.9 \%$ & $52.0 \%$ \\
Add only microgrid & $43.4 \%$ & $43.4 \%$ & $46.9 \%$ & $52.0 \%$ \\
Add only PV arrays & $2.4 \%$ & $46.9 \%$ & $46.9 \%$ & $52.0 \%$ \\
Add only wood-framed & $3.0 \%$ & $48.0 \%$ & $52.0 \%$ & $52.0 \%$ \\
buildings package & $0.1 \%$ & $43.6 \%$ & $47.1 \%$ & $52.2 \%$ \\
Add only SHW & & &
\end{tabular}

\subsubsection{Annual Energy Savings by Structure}

This section focuses on results from the 150-Soldier base camp at Fort Devens; however, the conclusions drawn may be applied to similar, larger base camps.

At Fort Devens, not all structures were modified to be energy efficient. For instance, the dining facility is a large, energy-intensive structure consisting of a tent and container that was not 
directly modified by SAGE technologies in the field; however, a portion of the dining facility's energy consumption was offset by the fact that it was connected to the microgrid and the PV array. The fuel savings targeted by SAGE are results of reducing the amount of electrical energy needed by a base camp and making the generation of that electrical energy more efficient.

Table 3-8 documents the annual energy demand for base camp structures in terms of source energy (i.e., gallons of fuel consumed by the generators), and in terms of energy use intensity (EUI). EUI is a commonly used metric to describe the energy performance of buildings. To determine EUI, the energy consumption of a building over a period is divided by that building's footprint or area (i.e., the building's consumption is normalized by its area). For instance, if a building consumes $500 \mathrm{kWh}$ annually and has a footprint of $100 \mathrm{ft}^{2}$, its EUI is $5 \mathrm{kWh} / \mathrm{ft}^{2} /$ year. By determining energy consumption per square foot, it is easier to compare the energy performance between different building types and sizes. For example, if Building A has an area of $100 \mathrm{ft}^{2}$ and consumes $500 \mathrm{kWh}$ while Building B has an area of $225 \mathrm{ft}^{2}$ and consumes 950 $\mathrm{kWh}$, it can be difficult to determine which building is holistically more efficient. However, the EUIs of Buildings $\mathrm{A}$ and $\mathrm{B}$ are $5 \mathrm{kWh} / \mathrm{ft}^{2} /$ day and $4.2 \mathrm{kWh} / \mathrm{ft}^{2} /$ day, respectively, and it becomes readily apparent that Building $B$ is more efficient.

Table 3-8. Annual Source Energy Savings by Structure

\begin{tabular}{lcccccc}
\hline & $\begin{array}{c}\text { Baseline } \\
\text { Kitchen and } \\
\text { Dining Tent/ } \\
\text { Container }\end{array}$ & $\begin{array}{c}\text { Baseline } \\
\text { Laundry } \\
\text { Container }\end{array}$ & $\begin{array}{c}\text { Baseline } \\
\text { Showers } \\
\text { Container }\end{array}$ & $\begin{array}{c}\text { Baseline } \\
\text { Latrines } \\
\text { Container }\end{array}$ & $\begin{array}{c}\text { Baseline } \\
\text { Air-Beam } \\
\text { Tent }\end{array}$ & $\begin{array}{c}\text { Energy } \\
\text { Efficient } \\
\text { Shelter }\end{array}$ \\
\hline \multicolumn{7}{c}{ Source Energy (gallons of fuel per year) } \\
\hline With Spot Generation & 17,187 & 13,336 & 14,666 & 11,036 & 5,472 & 3,170 \\
With Microgrid & 10,847 & 5,047 & 6,943 & 1,468 & 4,168 & 1,021 \\
Gallons Saved & 6,340 & 8,289 & 7,723 & 9,568 & 1,304 & 2,149 \\
Percent Savings & $36.9 \%$ & $62.2 \%$ & $52.7 \%$ & $86.7 \%$ & $23.8 \%$ & $67.8 \%$ \\
\hline \multicolumn{7}{c}{ Energy Use Intensity (gal/ft $\left.{ }^{2} / \mathbf{y r}\right)$} \\
\hline With Spot Generation & 22.6 & 111.5 & 9.7 & 46.1 & 8.6 & 5.0 \\
With Microgrid & 14.3 & 42.2 & 4.6 & 6.1 & 6.5 & 1.6 \\
Percent Savings & $36.9 \%$ & $62.2 \%$ & $52.7 \%$ & $86.7 \%$ & $23.8 \%$ & $67.8 \%$ \\
\hline
\end{tabular}

NOTE: The above figures were derived from the annual modeling of the entire 150-Soldier base camp at Fort Devens, based on data collected during field testing in Phases 2 and 3. 
The advantages of microgrids when compared to spot generation as well as the variation of savings by building type are evident in the calculated energy savings. For example, latrines and shelters reduced their fuel consumption by $86.7 \%$ and $67.8 \%$, respectively, while the air-beam tents (troop housing tent) and dining facility fuel consumption were reduced by smaller-but still significant-23.8\% and $36.9 \%$, respectively. The nature of these differences is due to the assumed Baseline conditions. The latrines and showers imposed relatively small loads on the Baseline $60 \mathrm{~kW}$ TQG used as spot generator, and as a result, the generators serving these structures ran at inefficient, low load levels. However, once integrated into a microgrid, these loads were provided with more efficiently produced electricity, which notably reduced the structure's source-energy consumption despite the fact the structure site-energy consumption remained unchanged (i.e., the structure itself did not consume more or less electrical energy). In the case of the dining facility, integration into the microgrid did reduce fuel consumption, but because the dining facility tends to operate at higher load levels, and thus loads its designated spot generator more efficiently, the percent savings of $36.9 \%$ was lower than that of the latrines.

This analysis leads to another important conclusion: even in cases where upgrading to a microgrid is not feasible, a reduction in the number of spot generators (where appropriate) has the potential to save significant amounts of fuel without added equipment cost. In such a situation, a careful analysis of each spot generator's load profile may reveal opportunities for improvement.

The load profile of each spot generator must be taken into account when viewing the EUI values to tell the whole, complex story. A single $60 \mathrm{~kW}$ spot generator serving the kitchen facility will be operating near peak efficiency, as the kitchen load closely matches the capacity of the generator. An identical $60 \mathrm{~kW}$ spot generator serving only the latrine facilities will be severely underloaded. A careful analysis of spot generation load profiles should be a first step in reducing fuel consumption at any base camp using spot generation.

Table 3-9 illustrates the savings that can be realized by both reducing the electrical energy required to operate a building and increasing the efficiency of the generation of that electrical energy. Specifically, Table 3-9 directly compares the Baseline structures operated on Baseline spot generation to a base camp with wood-framed structures operated on a microgrid. The smallest annual fuel savings values is $63.2 \%$ (kitchen facility) and the largest is $93.9 \%$ for the troop housing. EUI values that ranged from 8.6 to 111.2 gallons of fuel per square foot per year ( $\mathrm{gal} / \mathrm{ft}^{2} / \mathrm{yr}$ ) for the Baseline case range from 0.5 to $39.7 \mathrm{gal} / \mathrm{ft}^{2} / \mathrm{yr}$. These examples demonstrate that while a switch from spot generation to a microgrid benefits all structures in a base camp, a two-pronged approach combining more energy efficient structures with a higher efficiency electric power generation has higher potential for fuel savings. 
Table 3-9. Annual Source Energy Savings by Structure: Baseline vs. Wood-Framed Buildings

\begin{tabular}{|c|c|c|c|c|c|}
\hline Camp Structure & $\begin{array}{c}\text { Kitchen and } \\
\text { Dining } \\
\text { Facility } \\
\end{array}$ & Laundry & Showers & Latrines & $\begin{array}{c}\text { Troop } \\
\text { Housing }\end{array}$ \\
\hline \multicolumn{6}{|c|}{ Source Energy (gallons of fuel per year) } \\
\hline $\begin{array}{l}\text { Baseline with Spot } \\
\text { Generation }\end{array}$ & 17,187 & 13,336 & 14,666 & 11,036 & 5,472 \\
\hline $\begin{array}{l}\text { Wood-Framed with } \\
\text { Microgrid }\end{array}$ & 6,322 & 4,752 & 2,444 & 926 & 335 \\
\hline Gallons Saved & 10,865 & 8,584 & 12,222 & 10,110 & 5,137 \\
\hline Percent Savings & $63.2 \%$ & $64.4 \%$ & $83.3 \%$ & $91.6 \%$ & $93.9 \%$ \\
\hline \multicolumn{6}{|c|}{ Energy Use Intensity (gal/ft $\left.{ }^{2} / \mathrm{yr}\right)$} \\
\hline $\begin{array}{l}\text { Baseline with Spot } \\
\text { Generation }\end{array}$ & 22.6 & 111.5 & 9.7 & 46.1 & 8.6 \\
\hline $\begin{array}{l}\text { Wood-Framed with } \\
\text { Microgrid }\end{array}$ & 8.3 & 39.7 & 1.6 & 3.9 & 0.5 \\
\hline Percent Savings & $63.2 \%$ & $64.4 \%$ & $83.3 \%$ & $91.6 \%$ & $93.9 \%$ \\
\hline
\end{tabular}

\subsubsection{Annual Savings Worldwide}

This section presents the expected annual fuel and energy savings in fourteen different global locations for the 150-Soldier, 600-Soldier, and 5,000-Soldier base camps. The models for all base camp structures, PV arrays, and SHW system were combined with typical meteorological year (TMY) statistical weather data for each location. The savings shown in Figure 3-2 apply to fully implemented, best-case SAGE camps compared to a standard Baseline configuration for each base camp size.

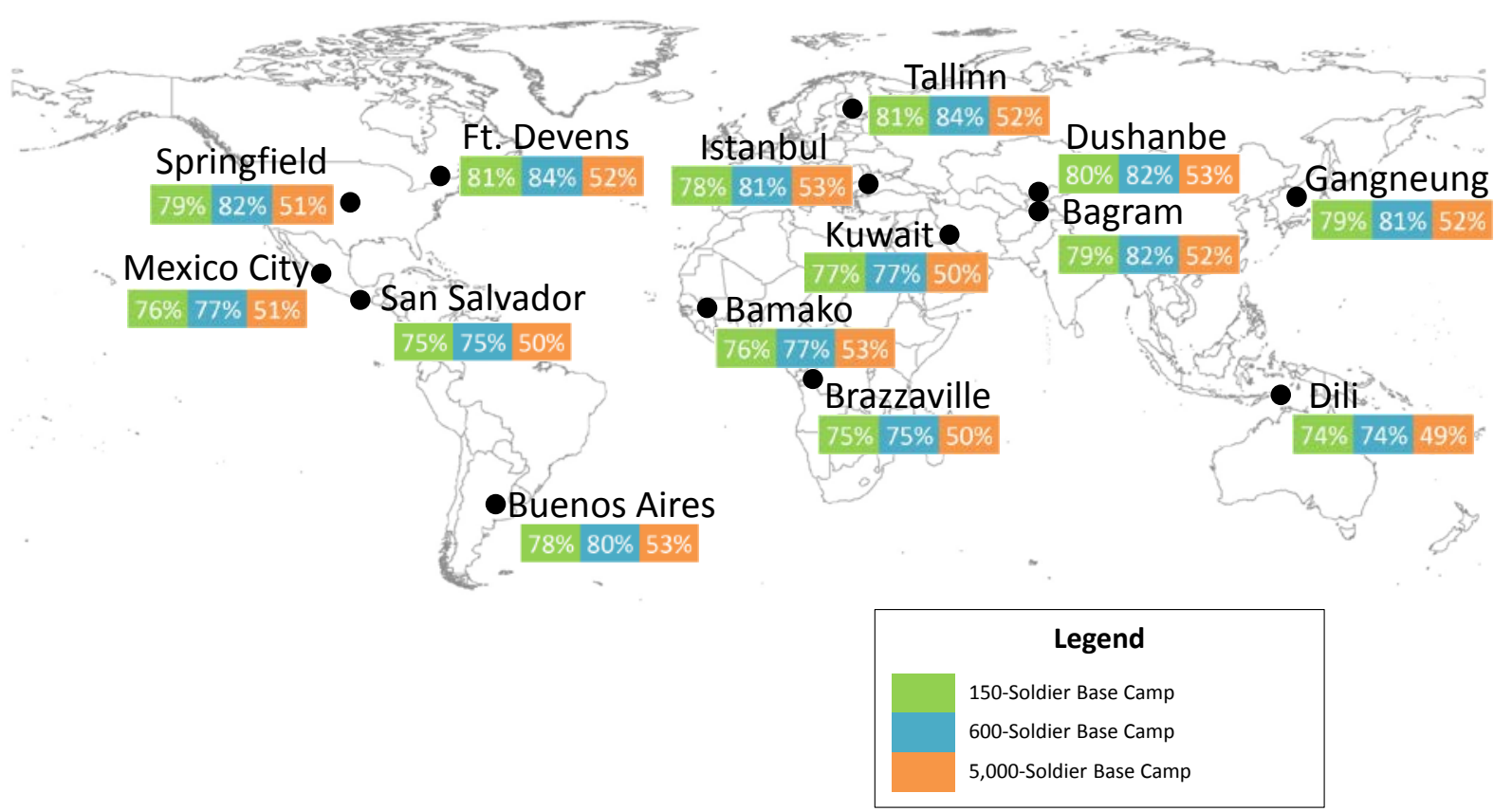

Figure 3-2. Global Annual Fuel Savings by Camp Size for all SAGE Technologies 
The fuel savings for the 150-Soldier base camps (green) range from 74\%-81\%. The global fuel savings for 600 -Soldier base camps (blue) ranged from $74 \%$ to $84 \%$. The global fuel savings for the 5,000-Soldier base camps (orange) ranged from $49 \%$ to $53 \%$.

For the 150-Soldier base camp, the reduction of fuel required for each location was similar in terms of percent, even though the Baseline fuel consumption for each location varied widely between 80,000 and 109,000 gallons. Locations with a smaller electrical footprint generally ran less-efficient spot generators due to lower-efficiency loading of those generators. A microgrid generated the power far more efficiently, but had to generate less of it as a consequence of the smaller loads.

Conversely, locations with a larger electric footprint — such as cold climate Talinn, Estonialoaded its spot generators more efficiently, making them perform far better than in locations with a smaller electric footprint. A microgrid running at the same efficiency would generate electric power with less of an improvement over spot generation. However, it could generate more power as a consequence of the larger electric footprint and thus the overall savings percentage would be similar in all locations.

Table 3-10 shows the detailed fuel savings percentages for seven different technology combinations in a 150-Soldier base camp in each location. The technologies are shown in the first four columns on the left, consisting of electricity generation (spot generation or microgrid), base camp buildings (Baseline or improved wood-framed buildings, and SAGE buildings to replace only the eight troop housing tents in only one case), water heating technology (standard or SHW), and renewable energy (PV, yes or no). Red fields indicate Baseline technology and green fields indicate SAGE technology. The first row below the headings represents a base camp with Baseline technology throughout. The bottommost row represents a base camp with SAGE technology throughout. All other rows show the possible remaining combinations.

Based on the energy modeling, the single largest improvement that can be made in 150 -Soldier base camps worldwide is the switch from the Baseline $6 \times 60 \mathrm{~kW}$ spot generation to the $3 \times 100 \mathrm{~kW}$ microgrid system, resulting in annual fuel savings ranging from $36 \%-59 \%$. The best combination of just two technologies is the microgrid together with replacement of the eight troop housing tents with SAGE energy efficient shelters, resulting in savings from $62 \%-66 \%$. 
Table 3-10. Detailed Global Fuel Savings for 150-Soldier Camps

\begin{tabular}{|c|c|c|c|c|c|c|c|c|c|c|c|c|c|c|c|c|c|}
\hline $\begin{array}{c}\text { Power } \\
\text { generation }\end{array}$ & Bldgs & $\begin{array}{c}\text { Water } \\
\text { heat }\end{array}$ & PV & Bagram & Bamako & Brazzaville & $\begin{array}{c}\text { Buenos } \\
\text { Aires } \\
\end{array}$ & Devens & Dili & Dushanbe & Gangneung & Istanbul & Kuwait & $\begin{array}{c}\text { Mexico } \\
\text { City }\end{array}$ & $\begin{array}{c}\text { San } \\
\text { Salvador }\end{array}$ & Springfield & Talinn \\
\hline $\begin{array}{l}6 \times 60 \mathrm{~kW} \\
\text { spot gen. }\end{array}$ & Base & std & no & $0 \%$ & $0 \%$ & $0 \%$ & $0 \%$ & $0 \%$ & $0 \%$ & $0 \%$ & $0 \%$ & $0 \%$ & $0 \%$ & $0 \%$ & $0 \%$ & $0 \%$ & $0 \%$ \\
\hline $\begin{array}{l}6 \times 60 \text { kW } \\
\text { spot gen. }\end{array}$ & Base & std & yes & $3 \%$ & $1 \%$ & $3 \%$ & $2 \%$ & $3 \%$ & $4 \%$ & $3 \%$ & $2 \%$ & $2 \%$ & $3 \%$ & $2 \%$ & $3 \%$ & $3 \%$ & $2 \%$ \\
\hline $\begin{array}{l}6 \times 60 \text { kW } \\
\text { spot gen. }\end{array}$ & Base & SHW & no & $1 \%$ & $1 \%$ & $1 \%$ & $1 \%$ & $1 \%$ & $1 \%$ & $1 \%$ & $1 \%$ & $1 \%$ & $2 \%$ & $1 \%$ & $2 \%$ & $1 \%$ & $1 \%$ \\
\hline $\begin{array}{l}3 \times 100 \mathrm{~kW} \\
\text { microgrid }\end{array}$ & Base & std & no & $44 \%$ & $59 \%$ & $59 \%$ & $53 \%$ & $36 \%$ & $53 \%$ & $44 \%$ & $44 \%$ & $48 \%$ & $52 \%$ & $58 \%$ & $59 \%$ & $43 \%$ & $33 \%$ \\
\hline $\begin{array}{l}3 \times 100 \mathrm{~kW} \\
\text { microgrid }\end{array}$ & Base & std & yes & $43 \%$ & $62 \%$ & $63 \%$ & $54 \%$ & $33 \%$ & $56 \%$ & $43 \%$ & $42 \%$ & $47 \%$ & $54 \%$ & $60 \%$ & $64 \%$ & $42 \%$ & $30 \%$ \\
\hline $\begin{array}{l}3 \times 100 \mathrm{~kW} \\
\text { microgrid }\end{array}$ & SAGE & std & no & $65 \%$ & $65 \%$ & $63 \%$ & $66 \%$ & $65 \%$ & $62 \%$ & $65 \%$ & $66 \%$ & $66 \%$ & $64 \%$ & $66 \%$ & $63 \%$ & $65 \%$ & $66 \%$ \\
\hline $\begin{array}{l}3 \times 100 \mathrm{~kW} \\
\text { microgrid }\end{array}$ & Imp. & SHW & yes & $79 \%$ & $76 \%$ & $75 \%$ & $78 \%$ & $81 \%$ & $74 \%$ & $80 \%$ & $79 \%$ & $78 \%$ & $77 \%$ & $76 \%$ & $75 \%$ & $79 \%$ & $81 \%$ \\
\hline
\end{tabular}


Table 3-11 shows the detailed fuel savings percentages for each possible technology combination in each location for 500-Soldier base camps. The technologies are shown in the first four columns on the left, consisting of electricity generation (spot generation or microgrid), base camp buildings (Baseline or improved wood-framed buildings), water heating technology (standard or SHW), and renewable energy (PV, yes or no). Red fields indicate Baseline technology and green fields indicate SAGE technology. The first row below the headings represents a base camp with Baseline technology throughout. The bottommost row represents a base camp with SAGE technology throughout. All other rows show the possible remaining combinations.

The addition of only SHW heating technology to a Baseline camp indicates fuel savings less than $0.5 \%$ annually. The SHW technology performs well when compared to standard fuel-fired water heating in isolation (generally $50 \%$ improvement or better). However, water heating requires very little fuel annually in relation to the annual fuel needs of the entire base camp, making even the best SHW system appear insignificant in terms of percent fuel saved overall. 
Table 3-11. Detailed Global Fuel Savings for 600-Soldier Camps

\begin{tabular}{|c|c|c|c|c|c|c|c|c|c|c|c|c|c|c|c|c|c|}
\hline $\begin{array}{c}\text { Power } \\
\text { generation }\end{array}$ & Bldgs & $\begin{array}{c}\text { Water } \\
\text { heat }\end{array}$ & PV & Bagram & Bamako & Brazzaville & $\begin{array}{c}\text { Buenos } \\
\text { Aires }\end{array}$ & Devens & Dili & Dushanbe & Gangneung & Istanbul & Kuwait & $\begin{array}{l}\text { Mexico } \\
\text { City }\end{array}$ & $\begin{array}{c}\text { San } \\
\text { Salvador }\end{array}$ & Springfield & Talinn \\
\hline $\begin{array}{l}24 \times 60 \mathrm{~kW} \\
\text { spot gen. }\end{array}$ & Base & std & no & $0 \%$ & $0 \%$ & $0 \%$ & $0 \%$ & $0 \%$ & $0 \%$ & $0 \%$ & $0 \%$ & $0 \%$ & $0 \%$ & $0 \%$ & $0 \%$ & $0 \%$ & $0 \%$ \\
\hline $\begin{array}{l}24 \times 60 \mathrm{~kW} \\
\text { spot gen. }\end{array}$ & Base & std & yes & $4 \%$ & $4 \%$ & $4 \%$ & $4 \%$ & $3 \%$ & $3 \%$ & $4 \%$ & $3 \%$ & $3 \%$ & $5 \%$ & $4 \%$ & $5 \%$ & $4 \%$ & $3 \%$ \\
\hline $\begin{array}{l}24 \times 60 \mathrm{~kW} \\
\text { spot gen. }\end{array}$ & Base & SHW & no & $0 \%$ & $0 \%$ & $0 \%$ & $0 \%$ & $0 \%$ & $0 \%$ & $0 \%$ & $0 \%$ & $0 \%$ & $0 \%$ & $0 \%$ & $0 \%$ & $0 \%$ & $0 \%$ \\
\hline $\begin{array}{l}24 \times 60 \mathrm{~kW} \\
\text { spot gen. }\end{array}$ & Base & SHW & yes & $4 \%$ & $5 \%$ & $4 \%$ & $4 \%$ & $3 \%$ & $4 \%$ & $4 \%$ & $3 \%$ & $4 \%$ & $5 \%$ & $4 \%$ & $5 \%$ & $4 \%$ & $3 \%$ \\
\hline $\begin{array}{l}24 \times 60 \mathrm{~kW} \\
\text { spot gen. }\end{array}$ & Imp. & std & no & $30 \%$ & $12 \%$ & $9 \%$ & $20 \%$ & $40 \%$ & $15 \%$ & $30 \%$ & $31 \%$ & $26 \%$ & $18 \%$ & $13 \%$ & $8 \%$ & $32 \%$ & $43 \%$ \\
\hline $\begin{array}{l}24 \times 60 \mathrm{~kW} \\
\text { spot gen. }\end{array}$ & Imp. & std & yes & $34 \%$ & $17 \%$ & $13 \%$ & $24 \%$ & $43 \%$ & $18 \%$ & $34 \%$ & $34 \%$ & $30 \%$ & $22 \%$ & $17 \%$ & $13 \%$ & $35 \%$ & $46 \%$ \\
\hline $\begin{array}{l}24 \times 60 \mathrm{~kW} \\
\text { spot gen. }\end{array}$ & Imp. & SHW & no & $30 \%$ & $13 \%$ & $10 \%$ & $21 \%$ & $40 \%$ & $15 \%$ & $30 \%$ & $31 \%$ & $27 \%$ & $18 \%$ & $14 \%$ & $9 \%$ & $32 \%$ & $43 \%$ \\
\hline $\begin{array}{l}24 \times 60 \mathrm{~kW} \\
\text { spot gen. }\end{array}$ & Imp. & SHW & yes & $34 \%$ & $17 \%$ & $14 \%$ & $25 \%$ & $43 \%$ & $18 \%$ & $34 \%$ & $34 \%$ & $30 \%$ & $23 \%$ & $18 \%$ & $13 \%$ & $36 \%$ & $46 \%$ \\
\hline $\begin{array}{l}4 \times 300 \mathrm{~kW} \\
\text { microgrid }\end{array}$ & Base & std & no & $38 \%$ & $56 \%$ & $58 \%$ & $49 \%$ & $30 \%$ & $52 \%$ & $38 \%$ & $38 \%$ & $42 \%$ & $48 \%$ & $55 \%$ & $59 \%$ & $37 \%$ & $26 \%$ \\
\hline $\begin{array}{l}4 \times 300 \mathrm{~kW} \\
\text { microgrid }\end{array}$ & Base & std & yes & $43 \%$ & $62 \%$ & $63 \%$ & $54 \%$ & $33 \%$ & $56 \%$ & $43 \%$ & $42 \%$ & $47 \%$ & $54 \%$ & $60 \%$ & $64 \%$ & $42 \%$ & $30 \%$ \\
\hline $\begin{array}{l}4 \times 300 \mathrm{~kW} \\
\text { microgrid }\end{array}$ & Base & SHW & no & $39 \%$ & $57 \%$ & $58 \%$ & $49 \%$ & $30 \%$ & $53 \%$ & $39 \%$ & $38 \%$ & $43 \%$ & $49 \%$ & $56 \%$ & $59 \%$ & $38 \%$ & $27 \%$ \\
\hline $\begin{array}{l}4 \times 300 \mathrm{~kW} \\
\text { microgrid }\end{array}$ & Base & SHW & yes & $43 \%$ & $62 \%$ & $63 \%$ & $54 \%$ & $34 \%$ & $56 \%$ & $43 \%$ & $42 \%$ & $47 \%$ & $54 \%$ & $61 \%$ & $65 \%$ & $42 \%$ & $30 \%$ \\
\hline $\begin{array}{l}4 \times 300 \mathrm{~kW} \\
\text { microgrid }\end{array}$ & Imp. & std & no & $77 \%$ & $72 \%$ & $69 \%$ & $74 \%$ & $80 \%$ & $70 \%$ & $77 \%$ & $77 \%$ & $76 \%$ & $71 \%$ & $72 \%$ & $69 \%$ & $77 \%$ & $81 \%$ \\
\hline $\begin{array}{l}4 \times 300 \mathrm{~kW} \\
\text { microgrid }\end{array}$ & Imp. & std & yes & $81 \%$ & $77 \%$ & $74 \%$ & $79 \%$ & $83 \%$ & $74 \%$ & $81 \%$ & $81 \%$ & $80 \%$ & $77 \%$ & $77 \%$ & $74 \%$ & $82 \%$ & $84 \%$ \\
\hline $\begin{array}{l}4 \times 300 \mathrm{~kW} \\
\text { microgrid }\end{array}$ & Imp. & SHW & no & $77 \%$ & $72 \%$ & $70 \%$ & $75 \%$ & $80 \%$ & $70 \%$ & $77 \%$ & $78 \%$ & $76 \%$ & $71 \%$ & $72 \%$ & $69 \%$ & $77 \%$ & $81 \%$ \\
\hline $\begin{array}{l}4 \times 300 \mathrm{~kW} \\
\text { microgrid }\end{array}$ & Imp. & SHW & yes & $82 \%$ & $77 \%$ & $75 \%$ & $80 \%$ & $84 \%$ & $74 \%$ & $82 \%$ & $81 \%$ & $81 \%$ & $77 \%$ & $77 \%$ & $75 \%$ & $82 \%$ & $84 \%$ \\
\hline
\end{tabular}


For the 5,000-Soldier base camps, savings are lower than in the smaller base camps, which is to be expected considering the far more energy-efficient Baseline technology of the larger, more permanent base camps. Expressed differently, the SAGE technologies-buildings, microgrids, renewable energy - perform as well as they do in smaller base camps, but the Baseline technology performs far better, narrowing the gap.

Detailed savings for 5,000-Soldier base camps in all locations are shown in Table 3-12. The technologies are shown in the first four columns on the left, consisting of electricity generation (spot generation or microgrid), base camp buildings (Baseline or improved wood-framed buildings), water heating technology (standard or SHW), and renewable energy (PV, yes or no). Red fields indicate Baseline technology and green fields indicate SAGE technology. The topmost colored row represents a base camp with Baseline technology throughout. The bottommost row represents a base camp with SAGE technology throughout. All other rows show the possible remaining combinations. 
Table 3-12. Detailed Global Fuel Savings for 5,000-Soldier Camps

\begin{tabular}{|c|c|c|c|c|c|c|c|c|c|c|c|c|c|c|c|c|c|}
\hline $\begin{array}{c}\text { Power } \\
\text { generation }\end{array}$ & Bldgs & $\begin{array}{c}\text { Water } \\
\text { heat }\end{array}$ & PV & Bagram & Bamako & Brazzaville & $\begin{array}{c}\text { Buenos } \\
\text { Aires }\end{array}$ & Devens & Dili & Dushanbe & Gangneung & Istanbul & Kuwait & $\begin{array}{c}\text { Mexico } \\
\text { City }\end{array}$ & $\begin{array}{c}\text { San } \\
\text { Salvador }\end{array}$ & Springfield & Talinn \\
\hline $\begin{array}{l}9 \times 3 \mathrm{MW} \\
\text { spot gen }\end{array}$ & Base & std & no & $0 \%$ & $0 \%$ & $0 \%$ & $0 \%$ & $0 \%$ & $0 \%$ & $0 \%$ & $0 \%$ & $0 \%$ & $0 \%$ & $0 \%$ & $0 \%$ & $0 \%$ & $0 \%$ \\
\hline $\begin{array}{l}9 \times 3 \mathrm{MW} \\
\text { spot gen }\end{array}$ & Base & std & yes & $3 \%$ & $2 \%$ & $2 \%$ & $2 \%$ & $2 \%$ & $2 \%$ & $3 \%$ & $2 \%$ & $2 \%$ & $3 \%$ & $2 \%$ & $2 \%$ & $2 \%$ & $2 \%$ \\
\hline $\begin{array}{l}9 \times 3 \mathrm{MW} \\
\text { spot gen }\end{array}$ & Base & SHW & no & $0 \%$ & $0 \%$ & $0 \%$ & $0 \%$ & $0 \%$ & $0 \%$ & $0 \%$ & $0 \%$ & $0 \%$ & $0 \%$ & $0 \%$ & $0 \%$ & $0 \%$ & $0 \%$ \\
\hline $\begin{array}{l}9 \times 3 \mathrm{MW} \\
\text { spot gen }\end{array}$ & Base & SHW & yes & $3 \%$ & $2 \%$ & $2 \%$ & $3 \%$ & $3 \%$ & $2 \%$ & $3 \%$ & $2 \%$ & $2 \%$ & $3 \%$ & $2 \%$ & $2 \%$ & $3 \%$ & $2 \%$ \\
\hline $\begin{array}{l}9 \times 3 \mathrm{MW} \\
\text { spot gen }\end{array}$ & Imp. & std & no & $3 \%$ & $3 \%$ & $3 \%$ & $3 \%$ & $3 \%$ & $4 \%$ & $3 \%$ & $3 \%$ & $3 \%$ & $4 \%$ & $3 \%$ & $3 \%$ & $3 \%$ & $3 \%$ \\
\hline $\begin{array}{l}9 \times 3 \mathrm{MW} \\
\text { spot gen }\end{array}$ & Imp. & std & yes & $6 \%$ & $5 \%$ & $5 \%$ & $5 \%$ & $5 \%$ & $5 \%$ & $6 \%$ & $5 \%$ & $5 \%$ & $6 \%$ & $5 \%$ & $5 \%$ & $5 \%$ & $5 \%$ \\
\hline $\begin{array}{l}9 \times 3 \mathrm{MW} \\
\text { spot gen }\end{array}$ & Imp. & SHW & no & $3 \%$ & $3 \%$ & $3 \%$ & $3 \%$ & $3 \%$ & $4 \%$ & $3 \%$ & $3 \%$ & $3 \%$ & $4 \%$ & $3 \%$ & $3 \%$ & $3 \%$ & $3 \%$ \\
\hline $\begin{array}{l}9 \times 3 \mathrm{MW} \\
\text { spot gen }\end{array}$ & Imp. & SHW & yes & $6 \%$ & $5 \%$ & $5 \%$ & $5 \%$ & $6 \%$ & $5 \%$ & $6 \%$ & $5 \%$ & $5 \%$ & $6 \%$ & $5 \%$ & $5 \%$ & $6 \%$ & $5 \%$ \\
\hline $\begin{array}{l}4 \times 3 \mathrm{MW} \\
\text { microgrid }\end{array}$ & Base & std & no & $44 \%$ & $44 \%$ & $43 \%$ & $45 \%$ & $43 \%$ & $41 \%$ & $44 \%$ & $45 \%$ & $45 \%$ & $41 \%$ & $45 \%$ & $43 \%$ & $44 \%$ & $44 \%$ \\
\hline $\begin{array}{l}4 \times 3 \mathrm{MW} \\
\text { microgrid }\end{array}$ & Base & std & yes & $48 \%$ & $49 \%$ & $46 \%$ & $48 \%$ & $47 \%$ & $44 \%$ & $48 \%$ & $48 \%$ & $48 \%$ & $45 \%$ & $48 \%$ & $46 \%$ & $47 \%$ & $48 \%$ \\
\hline $\begin{array}{l}4 \times 3 \mathrm{MW} \\
\text { microgrid }\end{array}$ & Base & SHW & no & $44 \%$ & $45 \%$ & $43 \%$ & $45 \%$ & $44 \%$ & $42 \%$ & $44 \%$ & $45 \%$ & $45 \%$ & $41 \%$ & $45 \%$ & $43 \%$ & $44 \%$ & $44 \%$ \\
\hline $\begin{array}{l}4 \times 3 \mathrm{MW} \\
\text { microgrid }\end{array}$ & Base & SHW & yes & $48 \%$ & $49 \%$ & $46 \%$ & $49 \%$ & $47 \%$ & $44 \%$ & $48 \%$ & $48 \%$ & $48 \%$ & $45 \%$ & $48 \%$ & $46 \%$ & $47 \%$ & $48 \%$ \\
\hline $\begin{array}{l}4 \times 3 M W \\
\text { microgrid }\end{array}$ & Imp. & std & no & $48 \%$ & $49 \%$ & $47 \%$ & $49 \%$ & $48 \%$ & $46 \%$ & $48 \%$ & $49 \%$ & $49 \%$ & $46 \%$ & $48 \%$ & $47 \%$ & $48 \%$ & $49 \%$ \\
\hline $\begin{array}{l}4 \times 3 \mathrm{MW} \\
\text { microgrid }\end{array}$ & Imp. & std & yes & $52 \%$ & $53 \%$ & $50 \%$ & $53 \%$ & $52 \%$ & $49 \%$ & $52 \%$ & $52 \%$ & $53 \%$ & $50 \%$ & $51 \%$ & $50 \%$ & $51 \%$ & $52 \%$ \\
\hline $\begin{array}{l}4 \times 3 \mathrm{MW} \\
\text { microgrid }\end{array}$ & Imp. & SHW & no & $48 \%$ & $49 \%$ & $47 \%$ & $49 \%$ & $48 \%$ & $46 \%$ & $48 \%$ & $49 \%$ & $49 \%$ & $47 \%$ & $48 \%$ & $47 \%$ & $48 \%$ & $49 \%$ \\
\hline $\begin{array}{l}4 \times 3 \mathrm{MW} \\
\text { microgrid }\end{array}$ & Imp. & SHW & yes & $52 \%$ & $53 \%$ & $50 \%$ & $53 \%$ & $52 \%$ & $49 \%$ & $53 \%$ & $52 \%$ & $53 \%$ & $50 \%$ & $51 \%$ & $50 \%$ & $51 \%$ & $52 \%$ \\
\hline
\end{tabular}





\subsection{System Reliability and Durability}

\subsection{Reliability of SAGE Components}

\subsubsection{Definition of Power Reliability and Power Quality}

Power reliability and power quality are important concepts since electrical equipment can be sensitive to poor power conditions. Power reliability refers to the robustness of the power system and its ability to provide power without outages. Power quality refers to the stability of certain electrical quantities in a power system. When large loads engage and disengage, they can momentarily affect the frequency and voltage of a grid. In extreme cases, poor power conditions can damage or destroy sensitive electronics. A stable grid is able to rapidly accommodate such deviations and protect downstream electrical equipment.

Power quality can be challenging at base camps. Large commercial grids have large generation capacities and a range of equipment to help maintain grid health. However, small grids, such as those at a base camp, are relatively susceptible to power quality issues given the base camp's limited generation capacity and the sophistication, or absence, of ancillary grid stabilization systems. Transitioning from spot generation to microgrids can help improve power quality and thus reduce problems and issues introduced by poor power quality.

\subsubsection{Microgrid vs. Spot Generation}

Networked power systems such as the SAGE microgrid possess inherent advantages in both power quality and reliability over spot generation. Should any generator in the microgrid fail, other generators and power sources (e.g., energy storage, renewable systems) can seamlessly accommodate the lost generation capacity. Conversely, a failed spot generator will immediately result in lost power, and power will not be restored until the generator is repaired or replaced. A malfunctioning spot generator may produce poor-quality power that could damage or destroy important mission-related equipment, which may be even more harmful than a short-term power outage.

Beyond failed or malfunctioning generators, additional issues are introduced by rapid changes in power demand. These rapid changes are often referred to as "transients" and can be produced when high capacity equipment such as large electric motors, electric food preparation equipment, or even coffee pots are turned on. With spot generation, a change in load levels is likely to be large relative to the generator capacity. For instance, if a $60 \mathrm{~kW}$ generator serves a building with a $5 \mathrm{~kW}$ griddle that is quickly turned on at full power; this griddle will demand nearly $10 \%$ of the generator capacity. Although $10 \%$ may seem modest, in an electric grid this is a large amount of variation that could occur in seconds. When such transients occur, they may produce over- or under-frequency and/or over- or under-voltage events, which, at best, trip circuits and cause black- or brownouts, or at worst, damage equipment. In a networked power system like the SAGE microgrid, a number of generators provide power to a number of loads. 
Any load transient is likely to be a small percentage of the total generating capacity, and as a result, the power system will be far more resilient than spot generation.

\subsubsection{Energy Storage System}

An energy storage system used in conjunction with a microgrid can further improve grid stability, reliability, power quality, and total system efficiency. In the event of generator failure, the energy storage system will immediately replace the lost generation capacity without requiring the start-up of another generator. The result is a power system that is more resilient to adverse situations than a spot generation or a small microgrid without an energy storage system.

Similarly, the energy storage system allows the microgrid generators to operate with less spinning reserve capacity than would be required otherwise. This is directly responsible for improved generator efficiency when compared to a microgrid without an energy storage system. In addition, the energy storage system provides power factor correction, i.e., the system helps to accommodate power loads that distort power quality across the entire microgrid, which further increases fuel efficiency of the generators.

\subsubsection{Renewable Energy}

Renewable energy systems can help reduce the fuel consumption of conventional base camp generators. For renewable energy systems to be effective, base camp planners should select the appropriate renewable system types and capacities that are best suited for the base camp location, renewable resource availability, operations tempo, and other constraints such as available space.

As renewable energy system capacity grows relative to base camp load levels, an energy storage system may be recommended. Many renewable resources such as wind and solar energy are not available on demand. Therefore, such renewable energy systems need to be carefully sized to avoid producing excess power when such power levels are not needed. Similarly, renewable systems need to be scaled to avoid introducing grid stability issues. As with transient loads, renewable energy systems introduce and remove large blocks of power generation capacity over short periods (e.g., the time during which a gust of wind blows or a cloud passes across the sun). These large power fluctuations can destabilize a grid unless additional equipment is present to act as a buffer and controller.

Energy storage systems can help address issues of both surplus power capacity and grid stability. For instance, if a PV array is sized such that it can produce excess energy during optimal periods, an energy storage system can store this energy and release it when the sun is not available. Similarly, an energy storage system can help buffer rapid changes in renewable system output that might be produced by a passing cloud, as it can rapidly release power and energy to the grid in response to the change in renewable system output.

\subsubsection{Base Camp Energy Management System}

A central base camp energy management system, such as the DSOM system used for the SAGE prototype, contributes to base camp reliability, fuel efficiency, operations security, and 
maintenance operations. DSOM can monitor base camp parameters (e.g., power generation, water flows and temperatures, and building temperatures) and help identify operations and maintenance issues before they become catastrophic. This capability improves problem response time, which contributes to the overall system reliability. Furthermore, central and/or remote monitoring of system parameters improves maintenance supervision, scheduling, and asset visibility, which leads to increased asset management control and cost reductions.

\subsubsection{SAGE Component Reliability Issues}

During SAGE testing, no generator or battery outages occurred but the test team did experience two minor component malfunctions. First, a ground-mounted fuel pump that supplied each generator with fuel malfunctioned and was quickly replaced. Second, a generator controller malfunctioned during load-bank testing but was quickly replaced by the vendor via overnight mail. When the SAGE team inquired into the normal lifetime expectancy of the controller, the vendor reported that the controller was designed for B10 life, a Six Sigma term that denotes the length of time between production and the point that $10 \%$ of the given product will fail. The B10 design requirement for the generator controller was 20,000 hours.

Additionally, the vendor reported that the engine mean time between failures (MTBF) for the commercial $100 \mathrm{~kW}$ generator was 7,347 hours. This figure was derived from a combination of the vendor's Quality, Reliability, Warranty and Analysis system and its Service Information Management system, which has tracked dealer-reported failures since 2007.

\subsubsection{SAGE SHW System Reliability Issues}

The SAGE SHW system was a drain-back system using an immersed load-side heat exchanger in a storage tank. Although it was a unique system developed and implemented for the SAGE demonstration, the SHW was designed with COTS components that are common to this type of domestic hot water system. Thus, domestic hot water MTBF data was included to provide an indication of the expected life of the collector and the overall system (see Table 4-1). 
Table 4-1. Mean Life of Major Elements in Flat-Plate Collectors

\begin{tabular}{|lc|}
\hline Elements & $\begin{array}{c}\text { Mean-Life Range } \\
\text { (years) }\end{array}$ \\
\hline Glazing & $>50$ \\
Glass & $5-10$ \\
Polymeric sheets & $2-5$ \\
Polymeric films & $1-10$ \\
Seals & $10-30$ \\
Insulation & $20-25$ \\
Absorber plate & \\
Absorber coatings & $1-5$ \\
Paints & $5-10$ \\
Black chrome & $15-30$ \\
Black chrome over dull nickel & \\
\hline
\end{tabular}

Table 4-2 ${ }^{12}$ indicates the average MTBF of a solar collector panel is from one to ten years under full load operating conditions. This MTBF is consistent with warranty data ${ }^{13}$ from most manufacturers and the SAGE SHW manufacturer that warrants its collectors to be free of defects for ten years from the date of installation.

Table 4-2. Collector Mean-Time-Between-Failure

\begin{tabular}{|c|c|c|c|c|c|c|c|c|c|}
\hline \multirow{3}{*}{$\begin{array}{l}\text { Number of } \\
\text { Collectors } \\
\text { in System }\end{array}$} & \multirow{3}{*}{$\begin{array}{c}\text { Approximate } \\
\text { Solar Load } \\
\qquad(\%)\end{array}$} & \multicolumn{4}{|c|}{$\begin{array}{c}\text { One-Tank Systems } \\
\text { Estimated MTBF (days) }\end{array}$} & \multicolumn{4}{|c|}{$\begin{array}{c}\text { Two-Tank Systems } \\
\text { Estimated MTBF (days) }\end{array}$} \\
\hline & & \multicolumn{2}{|c|}{$\begin{array}{c}\text { Without hose } \\
\text { interconnections }\end{array}$} & \multicolumn{2}{|c|}{$\begin{array}{c}\text { With hose } \\
\text { interconnections }\end{array}$} & \multicolumn{2}{|c|}{$\begin{array}{c}\text { Without hose } \\
\text { interconnections }\end{array}$} & \multicolumn{2}{|c|}{$\begin{array}{c}\text { With hose } \\
\text { interconnections }\end{array}$} \\
\hline & & Minimum & Maximum & Minimum & Maximum & Minimum & Maximum & Minimum & Maximum \\
\hline 2 & At least 50 & 227 & 1742 & 202 & 920 & 204 & 1325 & 174 & 768 \\
\hline 2 & 100 & 140 & 1431 & 124 & 800 & 130 & 1135 & 116 & 698 \\
\hline 4 & At least 50 & 219 & 1749 & 186 & 895 & 198 & 1329 & 171 & 770 \\
\hline 4 & At least 75 & 167 & 1623 & 148 & 870 & 155 & 1265 & 138 & 753 \\
\hline 4 & 100 & 95 & 1197 & 87 & 721 & 90 & 982 & 83 & 637 \\
\hline
\end{tabular}

The SAGE SHW system at the BCIL had a few minor piping leaks, power failure issues, drifting and/or failing sensors, a failed circulating pump (froze during transportation/storage

\footnotetext{
${ }^{12}$ Argonne National Laboratory. Final reliability and materials design guidelines for solar domestic hot water systems - ILL\# 100035939. ANL/SDP-11 Solar/0909-81/70, September 1981; Tables 2.9, and 3.7, Pages 33 and 160, "Estimated Mean Time between Failure (MTBF) of Indirect Drain-Back Solar DHW Systems."

${ }^{13}$ The results of a 2009 survey of 150 flat plate collector manufacturers and 50 vacuum tube collector manufacturers are depicted in Global Solar Thermal Energy Council. 2013. International Comparison of Warranty Periods granted by Collector Manufacturers. Accessed July 15, 2013 at http://solarthermalworld.org/content/internationalcomparison-warranty-periods-granted-collector-manufacturers.
} 
before initial start-up at BCIL), and an over-pressurization of the end-user potable water loop. The latter problem was caused because the SAGE SHW was designed with the intention that the SHW would be in regular operation such that this loop would always be open; however, during long periods of no use/demand, the loop system's valve was closed, which created excessive back-pressure requiring the addition of a pressure relief valve.

\subsection{Grid Stability Results}

Grid stability refers to voltage and frequency stability within the microgrid during both normal and abnormal operating conditions. In any power grid, frequency and voltage levels are invariably linked to both load conditions and generating conditions. Because electric power cannot be stored by the transmission/distribution system, the power of the generating sources must match the demand for power by the loads on the grid at any one time. Any mismatch will result in a tendency for instability in voltage and frequency. This phenomenon is rooted in the nature of the generating sources: nearly every source is a piece of rotating machinery. The frequency of the electric power produced is linearly linked to its mechanical rotation speed. If electric loads served by a generator fall below the current generator power output, the generator will speed up — as would any rotating machine with reduced load. Conversely, should the loads connected to it increase past the current power level of the generator, it will slow down. These variations are referred to as load transients and generation transients. A slowdown of the generator rotation speed causes a decrease in line frequency and voltage, while an increase of the generator rotation speed causes an increase in line frequency and voltage. It is desirable to have as little variation of voltage and frequency as possible.

A large power grid can deal with load variations and generator variations far better than a smaller grid. In a microgrid, there are typically a smaller number of generating sources and any sudden change in load is likely to be a large percentage of overall system load. As such, a microgrid is particularly susceptible to variations in line frequency and voltage. Many electric loads depend on a stable grid frequency of $60.0 \mathrm{~Hz}$ and voltage of $120 \mathrm{~V}$ (or perhaps $208 \mathrm{~V}$ or $480 \mathrm{~V}$ ). Frequent instability of these quantities may cause severely decreased equipment life, brownouts, blackouts, unstable speeds of electric motors, and general equipment malfunctions. Additionally, sudden instabilities may cause some loads and even generators to disconnect from the microgrid for self-protection, which may cause a cascading failure of the entire microgrid system.

\subsection{Grid Frequency Test Results}

The addition of an energy storage system to a microgrid provides a cushion of electric power without the burden of rotational speed. The SAGE energy storage system can almost instantaneously absorb additional demand from electric loads or absorb sudden additional power from the generators. Phase 1 testing specifically stressed the SAGE microgrid and energy storage system under these conditions to prove that the system could indeed improve grid stability. These tests-performed both with and without the SAGE energy storage systemincluded imposing large load transients and generation transients while monitoring both 
frequency and voltage response. Figure 4-1 shows the frequency stability of Phase 1 testing with and without energy storage.

\section{Micro-grid frequency response to large load transient}

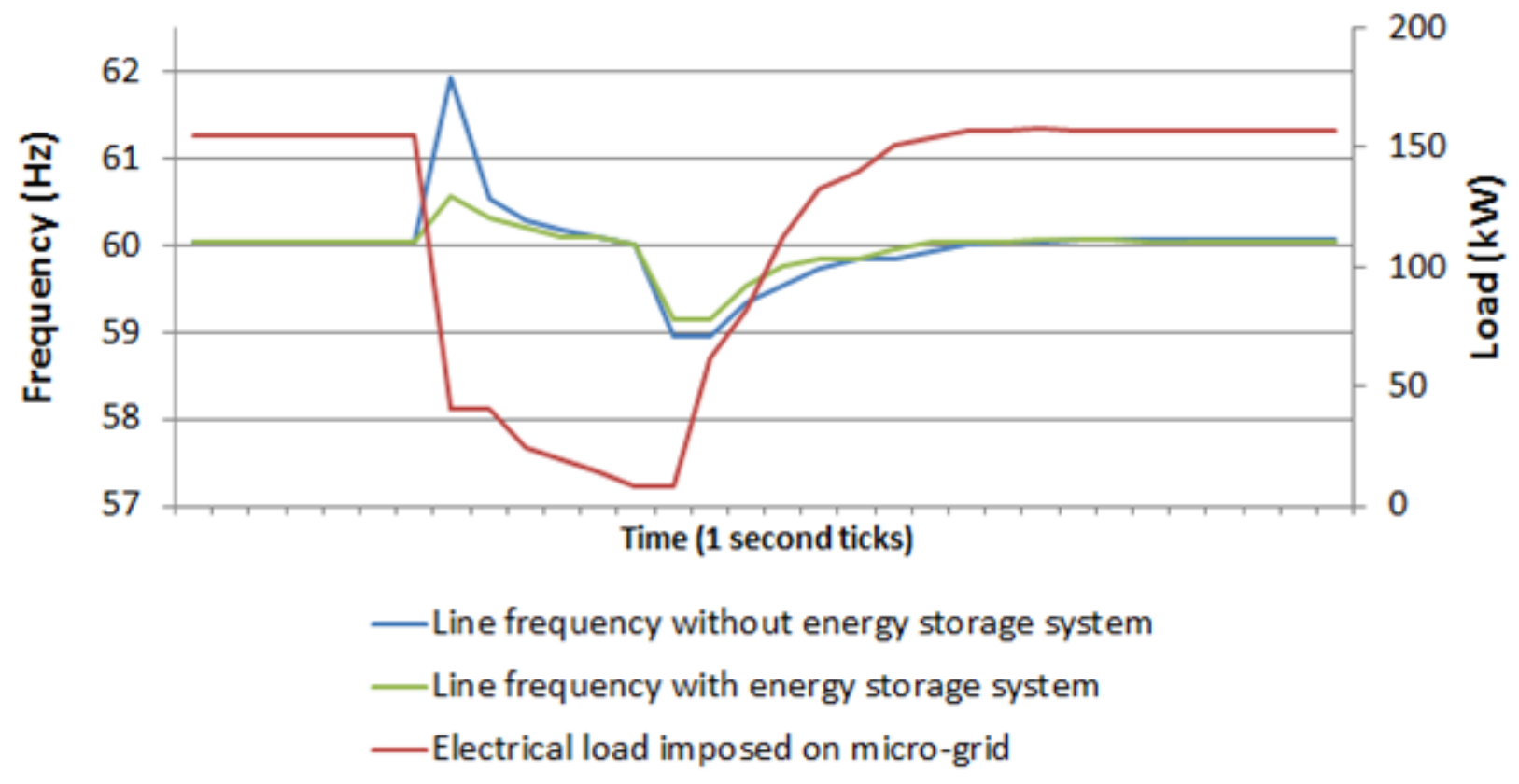

Figure 4-1. Microgrid Frequency Response to a Large Load Transient

Phase 2 tests did not aim to stress-test the microgrid in the same ways. However, voltage and frequency were monitored as part of the TIAX load profile testing and found to be well within allowable limits at all times.

This testing showed that the addition of an energy storage system to the SAGE microgrid improved grid stability. The SAGE microgrid performed as expected during all phases of testing and frequency and voltage stability were within the specifications designated for such a grid. 


\subsection{Lessons Learned}

The SAGE lessons learned focus on how to improve future SAGE-like analysis and recommended equipment improvements for future analysis.

\subsection{Testing Challenges}

As with any project of this size, challenges were encountered. This section serves to document those challenges and to offer insight into potential solutions for the future.

\subsubsection{Test Protocols}

Prepare test protocols in the early stages of a demonstration project. Include in the protocols alternative test concepts to address common problems in testing such as delayed equipment deliveries, difficult weather, and facility and equipment outages. Include time schedules for protocol development, testing periods, and the test report development.

Regularly review the developing and final protocols with stakeholders. Mock up the testing results to help stakeholders understand the testing goals and contribute to the protocols. Meet with the stakeholders at the testing facility to rehearse the testing steps, alternative protocols, and other procedures and authorities.

\subsubsection{Test Facility}

Confirm that the test bed for any new technology is mature and supported by established testing infrastructure. Test both wired and wireless networking for test computers and instrumentation for functionality under all conditions. Include the review of network security protocols and potential impacts on proposed testing.

\subsubsection{Instrumentation}

Discuss test instrumentation setups with the test bed operators early in the development of testing protocols to resolve any conflicts with existing test facility instrumentation and local laws and regulations. Select instrumentation to withstand any severe environmental conditions reasonably expected in the testing location.

\subsubsection{Testing Periods}

Plan for testing personnel to conduct testing 24/7 during the testing periods so that test delays and retesting can be accommodated. Plan for testing periods as long as can be accommodated by the project and testing facility. Include test site/facility support staff to operate systems not operable by the testing personnel. 


\subsubsection{Test Facility Infrastructure}

Provide a well-developed and easily accessible infrastructure. Include options for distribution level voltages of 480 to 4,160 V. Maintain calibration of water and fuel metering. Provide additional instrumentation as backup for when installed instrumentation appears to fail. Thoroughly document the configuration control and management for equipment and instrumentation. When installed site instrumentation is read remotely into a central computer, make the data easily accessible and document the data architecture and administration responsibilities.

\subsubsection{Soldier 'Testing'}

Select a testing facility with established patterns of Soldier occupancy, including being occupied by the same Soldiers for at least three seasons, and preferably all four seasons. Seek units that are flexible in their use of facilities so that end-user loads can be as similar as possible between the Baseline facilities and the improved facilities. Include in the test protocols a method acceptable to the stakeholders (units, Soldiers, facility management) for monitoring and documenting the Soldiers' facility use. Include in the protocols a method for obtaining feedback from the stakeholders during the testing period.

\subsubsection{Establish Baselines}

When planning field tests, select a facility that includes a portion that can remain unchanged during the testing period and serve as a baseline. Implement a configuration management plan to document the facility configuration and manage any changes.

\subsection{Recommended Equipment Improvements}

\subsubsection{Distribution Voltages}

Future microgrid projects should consider using distribution voltages higher than $208 \mathrm{~V}$, which was used for the SAGE prototype. Low-level voltage distribution is far more susceptible to line losses than a higher-level system. The distribution-line distances in the SAGE prototype were at the practical limit of a $208 \mathrm{~V}$ distribution. Small distribution transformers that step up voltage for distribution and step it back down for end use would be the easiest and the preferred solution to implement.

\subsubsection{Energy Storage System}

The SAGE energy storage system could benefit from lighter batteries, better charging efficiency, and smaller capacity. A lower weight could be achieved with different battery chemistry, such as lithium ion polymer. The energy storage system would maintain many of its advantages for power quality, stability, and efficiency even if it had less capacity than the SAGE system that was designed to provide two hours of backup power ("Silent Run" mode).

Furthermore, a reduction in capacity would reduce system weight and size, thus making it more 
practical for worldwide military deployments. Packaging energy storage systems in Tricon containers would be useful for the logistics/shipping systems used for 150- and 600-Soldier base camps.

\subsubsection{Renewable Energy}

The PV system would benefit from being more modular and containerized. It could also be beneficial to utilize roof-mounted arrays to avoid occupying valuable ground space. Packaging the PV and its support structure in Tricon containers would be useful for the logistics/shipping systems used for 150- and 600-Soldier base camps.

\subsubsection{Energy Efficient Shelters}

Setup for the hard-walled insulated SAGE shelters required approximately six to eight personnel and eight hours total. This setup could be made faster and performed with less manpower. Additionally, packaging unassembled shelters into Tricon or Bicon shipping containers would be advantageous from a logistical perspective.

\subsubsection{Solar Hot Water System}

SHW systems are highly configurable and adaptable to different installation environments and climates. The systems can be fully integrated and containerized to facilitate shipping and reduce operational footprints. However, there may be some trade-offs between keeping systems small enough to be containerized yet meeting hot water requirements. This size consideration also applies to the mounting the panels on the roof. Packaging the SHW system in Tricon containers would be useful for the logistics/shipping systems used for 150- and 600-Soldier base camps. These smaller Tricon containers can be bolted together to provide a larger, heavier base on which to install the SHW collectors.

The system is automated to operate in any climate without intervention. To make sure the systems do not go off line, managing undetected alarms should be considered. The alarm notification should also apply to a system operating out of control limits. Without the alarms or notification system, the backup system may take over the heating load and reduce or eliminate the energy savings.

\subsubsection{Energy Management System}

The base camp energy management system should be deployed more deeply to allow for more complete control and monitoring. The development focus should include defining and piloting the look and feel of the energy management system with users, and its form and functions with energy management system managers and designers. 


\subsubsection{Other Recommended Improvements}

Future implementations of SAGE or SAGE-like base camps should consider all technology available at that time. This may include fuel cells, other renewable energy sources such as wind power or high-efficiency PV, occupancy sensors, geothermal heat pumps, radiant heating and cooling, and solar shading technologies. A combination of multiple renewable energy technologies in a single base camp may enhance the availability of that energy. Perhaps most importantly, replacing all air-beam tents and all uninsulated base camp buildings with rigidwalled, insulated energy efficient buildings with high-efficiency heat pumps has the potential to decrease any base camp's energy footprint tremendously. 


\subsection{Conclusions and Recommendations}

The SAGE base camp fuel consumption was compared to that of the Baseline camp, which used spot generation, uninsulated tents with standard Army ECUs, and standard fuel-fired water heaters. Table 6-1 summarizes the field test results.

Table 6-1. Summary of Field Test Results

\begin{tabular}{ll}
\hline \multicolumn{1}{c}{ SAGE Technology } & \multicolumn{1}{c}{ Field Tests } \\
\hline $\begin{array}{l}\text { All SAGE technologies combined: } \\
\text { (Microgrid + renewable energy source } \\
\text { + energy efficient shelters + SHW } \\
\text { system) }\end{array}$ & Up to 54\% annual fuel savings \\
\hline $\begin{array}{l}\text { Microgrid + renewable energy system } \\
\text { (PV array and SHW) }\end{array}$ & Up to 41\% annual fuel savings \\
\hline Microgrid in isolation & Up to 34\% annual fuel savings \\
\hline Microgrid stability & $\begin{array}{l}\text { Microgrid delivered power with stable voltage and frequency } \\
\text { without any power outages or phase distortions. }\end{array}$ \\
\hline Silent run capability & $\begin{array}{l}\text { The energy storage battery system silently powered a 100 kW } \\
\text { base camp load without generators for two hours. }\end{array}$ \\
\hline Energy efficient shelters & $\begin{array}{l}\text { Shelters demonstrated an average of 85\% less energy than tents } \\
\text { and exhibited superior temperature stability. }\end{array}$ \\
\hline $\begin{array}{l}\text { Base camp energy management } \\
\text { system }\end{array}$ & $\begin{array}{l}\text { System enabled central monitoring and control of vital base } \\
\text { camp equipment and functions, and could feature automated } \\
\text { load reduction schemes. }\end{array}$ \\
\hline PV array & $\begin{array}{l}\text { The deployed renewable energy power system produced up to } \\
12 \% \text { of daily base camp energy needs. }\end{array}$ \\
\hline $\begin{array}{l}\text { Solar hot water system } \\
\text { (a) Based on Phase 2 and Phase } 3 \text { field tests; Phase } 3 \text { test results were normalized using gallons consumed because } \\
\text { the occupancy of the Baseline camp was significantly lower than that of the SAGE camp. }\end{array}$ \\
\hline
\end{tabular}


EnergyPlus models were developed for different base camp sizes and global locations. The models were calibrated using the field test data. The modeled savings varied by location and camp size; however the greatest energy savings were consistently to replace spot generation with a smart microgrid and replace Baseline buildings with wood-framed, insulated buildings with high-efficiency heat pumps. Figure 6-1 shows the savings associated with the implementation of all SAGE technologies ranged from $49 \%$ to $84 \%$ depending on location. The savings were influenced by climate, the Baseline building efficiency, and how the energy load profile affected the microgrid efficiency.

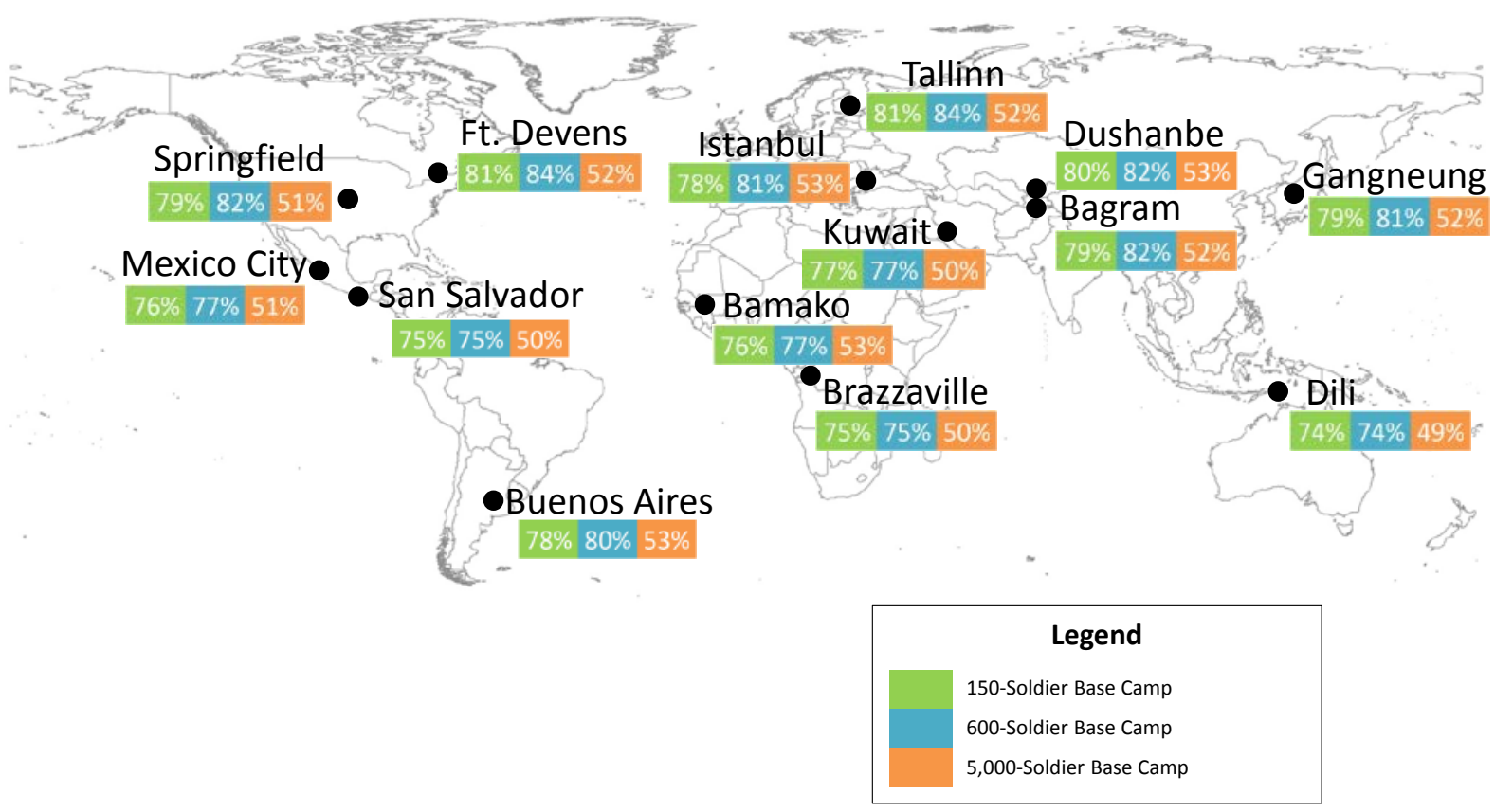

Figure 6-1. Global Annual Fuel Savings by Camp Size for all SAGE Technologies 
Five key conclusions from the analysis of SAGE technologies are summarized in Table 6-2.

Table 6-2. Conclusions

Efforts to reduce fuel consumption need to include reducing base camp electric energy consumption and generating this electric energy as efficiently as possible.

A combination of energy conservation measures, renewable energy sources, and a smart microgrid can reduce base camp energy consumption by $49 \%-84 \%$ depending on camp size and location. Smart microgrids with energy storage systems supply power with improved voltage and frequency stability, increased grid reliability and longer life of end-use equipment. If microgrids are not possible, right-sizing of existing spot generation has the potential to save significant amounts of fuel annually.

Renewable energy systems can reduce base camp energy demand and fuel use. They can be reliably integrated into smart base-camp microgrids without harming grid stability or degrading the output of the renewable source. A combination of spot generation with renewable energy sources is generally not recommended.

A properly sized SHW heating system uses solar energy to supply a significant fraction of a base camp's water heating requirements, thereby saving fuel over standard water heating configurations.

A base camp energy management system, equipped with real-time monitoring and control of base parameters, enables central and informed decision making without overburdening the operator with information. Configurable automatic load shedding provides potential for reducing base camp energy consumption for normal operations and for unplanned events.

The following SAGE team recommendations are listed in order of importance when making investment decisions to upgrade or construct more energy efficient base camps:

1. Replace spot generation systems with properly sized power generators that are integrated with smart microgrids. When appropriate, include an energy storage system and a base camp energy management system. When a microgrid is not initially feasible, correctly size the spot generators to meet the actual loads.

2. Replace poorly or un-insulated tents with insulated, energy efficient shelters featuring rightsized high-efficiency ECUs. Add insulation to uninsulated shelters such as wood-framed or concrete block shelters. Include remotely controllable circuits and integration with a base camp energy management system.

3. Install a well-designed and right-sized sized SHW system in camps with suitable conditions (e.g., expensive energy, remote areas, strong solar resource, high-volume water heating requirements, etc.).

4. Implement a base camp energy management system that features remote control capability of electric loads, reporting, and decision support and can feature automated load reduction schemes.

5. Implement right-sized renewable energy sources suitable for the base camp climate conditions and make sure the renewable energy system can integrate into a microgrid and base camp energy management system. 



\section{Appendix A \\ SAGE Technology Guide Specifications}

\section{Purpose}

These Smart and Green Energy (SAGE) guide specifications provide the key or salient features of facilities and products that will implement the technologies demonstrated by SAGE to improve tactical operations and to maximize fuel savings. The SAGE set of technologies includes the following major systems:

- smart microgrid generators and battery storage

- renewable energy generation, such as photovoltaic (PV) power and solar hot water heating

- insulated facilities with commercially available high efficiency heating and cooling heat pumps

- remotely controllable facility equipment, such as circuit breakers, thermostats, and lights, to enable load shedding

- facility/utility monitoring and control systems

The project designer's integration of these technologies into a project will provide many benefits including the following:

- significantly reduce fuel consumption and the frequency of fuel transports

- employ energy storage to reduce fuel use and improve electrical grid health to the level of a public utility

- employ and optimize renewable energy sources

- provide tactical monitoring and control of facilities by individuals located at the camp and/or a remote location

- improve contingency responses by shedding electrical loads in a prioritized manner

- provide accurate fuel supply status and support the planning for fuel resupplies

- improve planning and deployment of a consistent and scalable generation design as base camps grow or decrease

- reduce equipment maintenance and unplanned outages, and extend equipment life.

These specifications do not address cost-effectiveness because the project economic justification for a particular project is unique to that project.

\section{A.1.1 Background}

The U.S. Army Logistics Innovation Agency (LIA) requested the U.S. Department of Energy's Pacific Northwest National Laboratory (PNNL) to demonstrate how commercial off- 
the-shelf (COTS) technologies could reduce the fuel use of Forward Operating Bases. This demonstration project, known as SAGE, designed, deployed, and operated the set of COTS technologies at the Base Camp Integration Laboratory (BCIL) in Fort Devens, Massachusetts. SAGE provides these specifications based on the BCIL field tests and additional information. Renewable energy, solar hot water, smart microgrid, and energy efficient technologies are advancing rapidly. When developing specifications check the technical literature to make sure the latest technology is considered.

SAGE directs these specifications to facility designers, project managers, purchasing agents, contractors, vendors, and manufacturers. The specification format follows the construction specification industry's simplified writing style of stating requirements, options, and guidance and with minimal instruction or guidance on which role shall implement one or more of the specification requirements. SAGE's recommended requirements are stated as tasks, such as "Provide a smart generator controller" and "Include a vestibule." SAGE's suggestions and guidance are stated as "Consider," "Recommend," and "For reference." Each organization determines how to assign these tasks for design, fabrication, and/or construction within its standard conduct of operations.

Each organization also determines what codes and standards are appropriate. Given the large body of existing design and construction standards, the SAGE specifications do not address codes and standards requirements except where the reference may be key to implementation.

The U.S. Department of Defense (DOD) maintains a library of facility design standards called the Unified Facilities Criteria (UFC) that guide project designers and managers. DOD also maintains a large library of detailed construction specifications called the Unified Facilities Guide Specifications (UFGS) that contractually direct contractors and vendors. The UFGS specifications are similar to the construction industry's detailed specifications used for construction contract documents and bidding. UFC and UFGS include requirements for complying with building and manufacturer codes and standards. Each SAGE specification section notes several of the UFCs and UFGS that directly relate to the SAGE technology.

SAGE's stakeholders included the U.S. Army Office of the Chief of Engineers, which manages the content and implementation of the Army Facilities Components System (AFCS) that governs the planning, design, acquisitions, and construction of facilities in Theaters of Operation. The AFCS key documents include standard or generic designs for more than 200 site configurations and more than 1,000 variations of facilities. The AFCS standards include standard specifications in the form of Theater Oriented Guide Specifications (TOGS) and National Stock Numbers (NSNs), as well as specification narrative placed on standard construction drawings. The TOGS are typically abbreviated versions of the UFGS specifications.

The NSNs define a single facility component (such as a nail), or an assembly (such as an air conditioner). The NSNs are written and regulated at the national, federal level by the Defense Logistics Agency. However, AFCS engineers write a large quantity of NSNs for 
use only within AFCS projects; these internal NSN numbers contain "ZZ" to identify them as created and managed by AFCS.

The AFCS developed a large, integrated project development system named the Theater Construction Management System (TCMS) that is becoming the Joint Construction Management System (JCMS) for use by all military branches. Each SAGE specification section provides guidance on how to incorporate SAGE specifications into existing specification systems.

Each SAGE specification section includes information that may be repeated from the report body because this Appendix may be distributed separately from the report.

Each SAGE specification section includes manufacturer product information on the equipment demonstrated by SAGE at the Army's BCIL at Fort Devens, Massachusetts. This information is not an endorsement of the manufacturers or the models, but is to provide examples of products that can meet the function described in the specification. In the language of the specification industry, these products can be used as "or equal" references.

\section{A.1.2 Scalability of SAGE Technologies}

SAGE's purpose was to demonstrate that systems designed with COTS technologies can significantly reduce fuel use in all sizes of Forward Operating Bases, whether the bases already exist or are in planning. SAGE's demonstration facility was a 150-Soldier base camp that relied on spot generation for electricity. As existing base camps increase in population and permanence, spot generation is gradually replaced by large, centralized power plants. New large base camps are deployed with central power plants. With the increasing scale of base camps in mind, each SAGE specification section includes guidance on how to apply SAGE to larger base camps. The most complex scaling options are for the microgrid and battery technologies. For the other SAGE technologies, scalability is the simpler multiplication of the technology units, such as adding additional PV arrays until the desired capacity is achieved.

\section{SAGE Base Camp Smart Microgrid}

\section{A.1.3 Description}

The SAGE smart microgrid integrates power equipment to provide grid reliability, stable voltage and frequency, and optimal use of renewable energy sources such as PV and wind power. The microgrid consists of smart grid power-generating technologies connected on a parallel bus to reduce fuel use and to improve tactical control. The microgrid includes a common control system to network multiple power generators and to connect with any available renewable energy and energy storage sources.

The key microgrid technology is a smart generator controller that runs generators in the most economical manner to complement the constantly fluctuating electrical loads of a base camp. 


\section{A.1.4 Specifications}

\section{A.1.4.1 Generators}

- Provide commercially available prime generators that have paralleling capabilities.

- Consider incorporating radio interference protection requirements to the existing specifications to protect equipment and cable. Consider using the International Electrotechnical Commission (IEC) 61000-6 or the EN61000-6 standards regarding electromagnetic compatibility (EMC) - Part 6-1: Generic standards - Immunity for residential, commercial and light-industrial environments.

- Provide open communication and control protocols, such as but not limited to the following:

- CAN (controller area network [automotive industry])

- RS-485 annunciator data line

- Modbus TCP (Transmission Control Protocol), 10BT Ethernet

- Modbus RTU (remote terminal unit), RS-485 half duplex

- BACnet (Building Automation and Control Networks)

\section{A.1.4.2 Smart Controllers}

Provide a generator controller to perform the following tasks:

- Run, stop, and automatic-mode controls

- Parallel generation control to network generators

- automatic adjustment of generator speed and voltage

- remote start, stop, and emergency stop of the generator set

\section{A.1.4.3 Generator Monitoring}

- Provide the following generator monitoring functions:

- voltage $(\mathrm{L}-\mathrm{L}, \mathrm{L}-\mathrm{N})^{14}$

- current (phase)

- average volts, amps, and frequency

- $\mathrm{kW}, \mathrm{kVAr}$, and kVA (average, phase, percent)

- power factor (average, phase)

- $\mathrm{kW}-\mathrm{hr}$ and kVAr-hr (total)

- excitation voltage and current

${ }^{14} \mathrm{~L}-\mathrm{L}=$ line-to-line; $\mathrm{L}-\mathrm{N}=$ line-to-neutral 
- generator stator and bearing conditions

- Provide onboard system diagnostics.

- Provide front-panel keyboard access to monitoring and controls:

- detect and manage reverse power $(\mathrm{kW})$

- detect and manage reverse reactive power (kVAr)

- detect and manage overcurrent

- balance currents

\section{A.1.4.4 Generator Protection}

- Provide the following automatic functions either with the generator or through the controller:

- detect and manage over/under voltage

- detect and manage over/under frequency

\section{A.1.4.5 Communication}

- Provide open communication and control protocols, such as but not limited to one of the following:

- CAN

- RS-485 annunciator data link

- Modbus TCP (10BT Ethernet)

- Modbus RTU (RS-485 Half duplex)

- BACnet

\section{A.1.4.6 Electrical Distribution}

The following bullets summarize several standard TCMS requirements for general electrical distributions that also are important to a microgrid:

- Provide an electrical distribution system that connects base camp facilities to the generator bus.

- Provide properly distributed and rated cable for voltage throughout the base camp, and eliminate voltage drop to the loads.

- Provide appropriately rated transformers to step up the generator output to the base camp distribution voltage.

- Size the transformers to step down the distribution bus at the various electrical panels that feed the base camp loads and facilities. 
- Provide standard switchgear to synchronize and protect interconnections of the microgrid with adjacent and base-camp-wide grids.

Refer to the SAGE Contingency Base Energy Management System specification for additional requirements and considerations, and for integrating the microgrid with base camp operations.

\section{A.1.5 Scalability}

- Consider the following two options for smart microgrid deployments for base camps larger than the 150-Soldier size:

- Deploy multiple smart microgrids, centralized at each battalion, or

- Deploy larger prime power generators (400 kW to $2 \mathrm{MW}$ ) at one location, or at two locations if desired, for security and redundancy.

- Provide the standard TCMS $13.8 \mathrm{kV}$ power distribution with matching transformers.

- Consider the following microgrid designs for three scales of base camps: 150-, 600-, and 5,000-Soldier base camps. Figure A-1 illustrates the key design concepts for each size of base camp.

\section{A.1.5.1 150-Soldier Base Camp Smart Microgrid}

- Provide multiple smaller generators with the smart grid technologies to provide the total power generation capability of $240 \mathrm{~kW}, 3$ phase, 208/480 VAC $(240 \mathrm{~kW}$ at 0.8 power factor). Install smart controllers in existing generators or in generators that are purchased locally.

For reference, the existing TCMS design for a 150-Soldier base camp is six $60 \mathrm{~kW}$ Tactical Quiet Generators (TQG-60) wherein each TQG is operated as a stand-alone (a.k.a. spot) generator that serves a limited number of facilities. Spot generator design does not allow for the generators to be operated in the integrated microgrid strategy that SAGE field tests have demonstrated to save significant fuel. In addition, spot generation cannot reliably accommodate renewable energy sources such as PV solar and wind power because of the variable loads on a generator and the variable output of PV and wind power.

\section{A.1.5.2 600-Soldier Base Camp Smart Microgrid}

- Provide three $500 \mathrm{kVA}$ smart generators to meet the entire base camp electrical load. If a base camp requires two distribution systems, install two sets of three $200 \mathrm{~kW}$ smart generators. Install smart controllers in existing generators or in generators that are purchased locally.

For reference, the current TCMS design shows four $500 \mathrm{kVA}(400 \mathrm{~kW})$ generators at a 600-Soldier camp. As an alternative, six $200 \mathrm{~kW}$ smart generators can be used to power the entire base camp. 


\section{Smart Microgrid scaling from 150 Soldier camp to $\mathbf{5 , 0 0 0}$ Soldier camp}

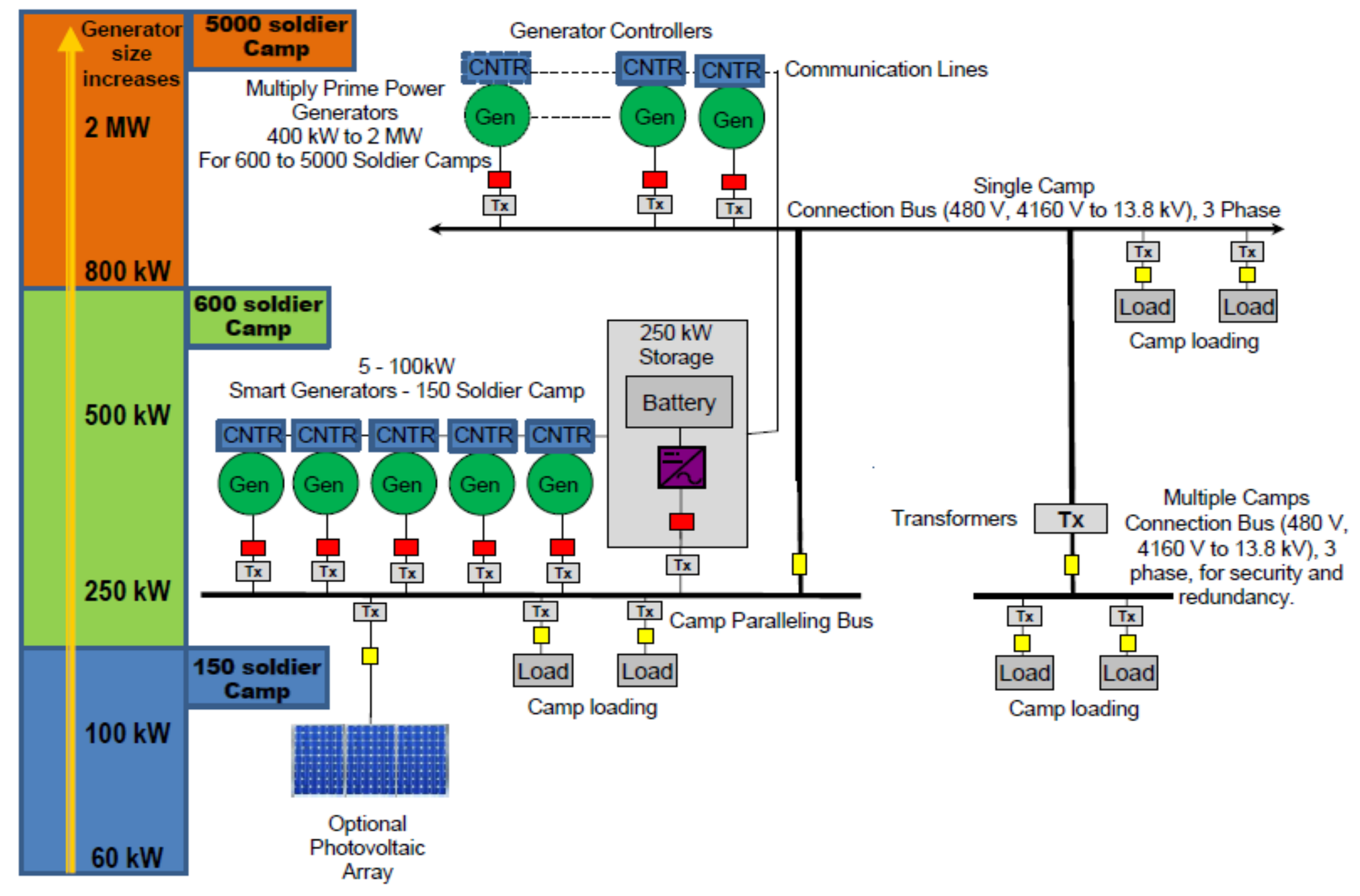

Figure A-1. Microgrid Scaling from 150-Soldier Camp up to 5,000-Soldier Camp 


\section{A.1.5.3 5,000-Soldier Base Camp Smart Microgrid}

- Consistent with the TCMS existing design concept for a large base camp, provide two power plants with parallel connected generators, sized at 1 or $2 \mathrm{MW}$, or a combination of sizes, selected by evaluating the base camp's load profile. Provide a ring-bus distribution for flexibility and control. Install smart controllers in existing generators or in generators that are purchased locally.

For reference, the current TCMS design shows two power plants with a total of eleven generators capable of generating up to $15,405 \mathrm{kVA}$. One generator power plant, for example, shows two 1,000 kVA $(800 \mathrm{~kW})$ units and three 2,000 kVA $(1600 \mathrm{~kW})$ units.

\section{A.1.6 References}

\section{A.1.6.1 Unified Facilities Criteria}

- UFC 3-501-01, Electrical Engineering

- UFC 3-540-04N, Diesel Electric Generating Plants ${ }^{15}$

- UFC 3-550-01, Exterior Electrical Power Distribution

- UFC 3-560-01, Electrical Safety, O\&M; with Change 4

\section{A.1.6.2 Unified Facilities Guide Specifications}

- UFGS 260571.00 40, Low-Voltage Overcurrent Protective Devices

- UFGS 2609 13, Power Monitoring System

- UFGS 2611 14.00 10, Main Electric Supply Station and Substation

- UFGS 2612 19.10, Three-Phase Pad-Mounted Transformers

- UFGS 262714.00 20, Electricity Metering

- UFGS 263214.00 10, Diesel-Generator Set, Stationary 15-300 kW, Standby Applications

- UFGS 263215.00 10, Diesel-Generator Set Stationary 100-2500 kW, with Auxiliaries

- UFGS 263623.00 20, Automatic Transfer Switches

- UFGS 264100.00 20, Lightning Protection System

\section{A.1.7 Incorporate SAGE Technologies into Specifications}

- Revise the existing specifications for generators to include the paralleling generator and the smart controller specifications, and the central plant improvements.

\footnotetext{
${ }^{15}$ This generating plant spec has the key microgrid functions such as paralleling controls.
} 
The proprietary smart controller demonstrated during SAGE field tests may be useful to include as an "or equal" reference in the appropriate specifications, such as TCMS TOGS and ZZ-NSNs, for the purpose of defining the quality of product and the range of functions. The SAGE controller was the latest version, Version 4.4, of Caterpillar's EMCP smart controller product line. Caterpillar installed the controller into SAGE's three Caterpillar generators and into SAGE's TQG-60 provided through the LIA. The controller was successfully demonstrated in the SAGE demonstration, working uniformly in the Caterpillar and TQG generators. Write the specification revisions or new sections to focus on the nonproprietary requirements and include a statement like "Provide a Caterpillar EMCP 4.4 controller or equivalent."

\section{A.1.8 Key Equipment Deployed in the SAGE Demonstration at BCIL}

The SAGE BCIL generator smart control was the Caterpillar EMCP 4.4 Generator Set Controller. Additional information is at the following link: http://www.cat.com/powergeneration/emcp.

The SAGE BCIL generators were three Caterpillar XQ100 Rental $100 \mathrm{~kW}$ with trailer, and one standard TQG-60, skid-mounted, $60 \mathrm{~kW}, 50 / 60 \mathrm{~Hz}, \mathrm{MEP}-806 \mathrm{~A}$. The TQG-60 is also available as NSN 6115-01-274-7390, End Item Code VG7. The TQG was provided by the Army and Caterpillar replaced the TQG's stock controller with the EMCP 4.4 to integrate the TQG into the microgrid.

The SAGE BCIL power distribution system consisted of three Lex Mobile Power distribution boxes, each 1200 amp, 3 phase, 4 pole, 5 wire, 120/208 Y VAC, 50/60 Hz; catalog number DB350NA-P2WQS3, NSN-6110-01-352-1776, General Services Administration (GSA) \# GS074-0296N.

The following diagram, Figure A-2, illustrates the Physical Layout of the SAGE Equipment at BCIL, Including the Energy Storage (battery) System, and PV Panels. 


\section{PNNL - SAGE 150 Man FOB Micro-grid Test Bed}

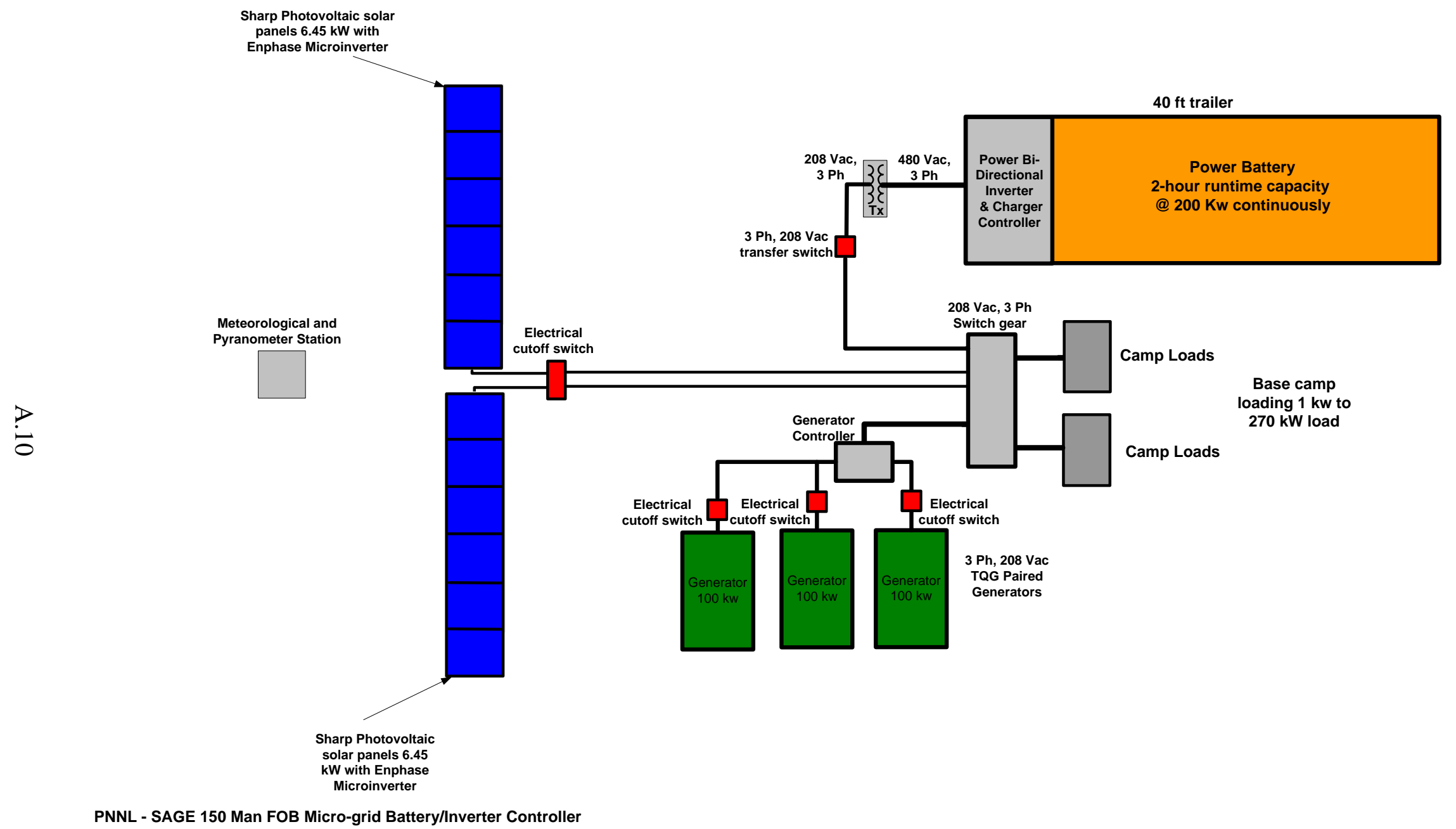

Figure A-2. Diagram Illustrating the Physical Layout of the SAGE Equipment at BCIL, Including the Energy Storage (battery) System, and PV Panels 


\section{A.1.9 Photos of the Deployed SAGE Systems at BCIL}

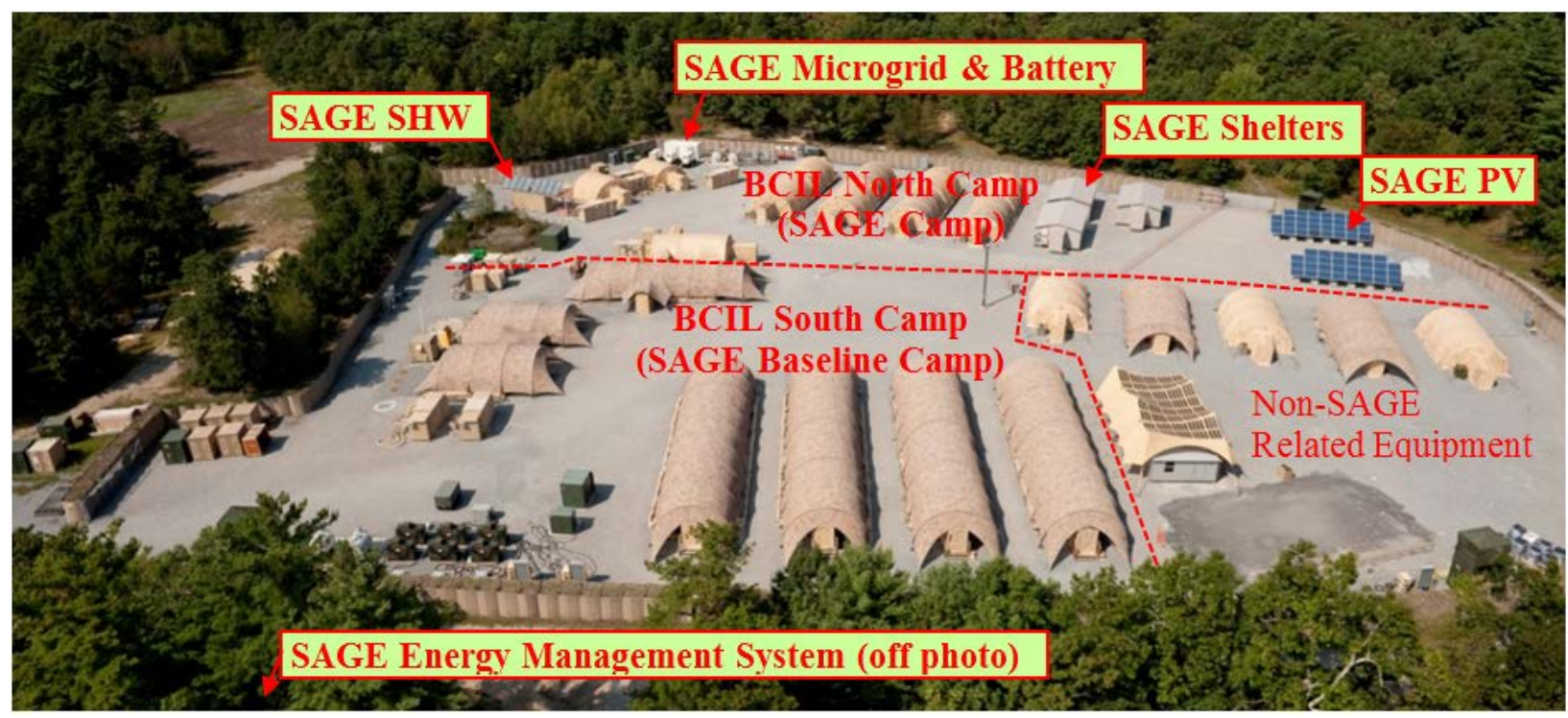

Figure A-3. The Physical Layout of the SAGE Equipment at BCIL

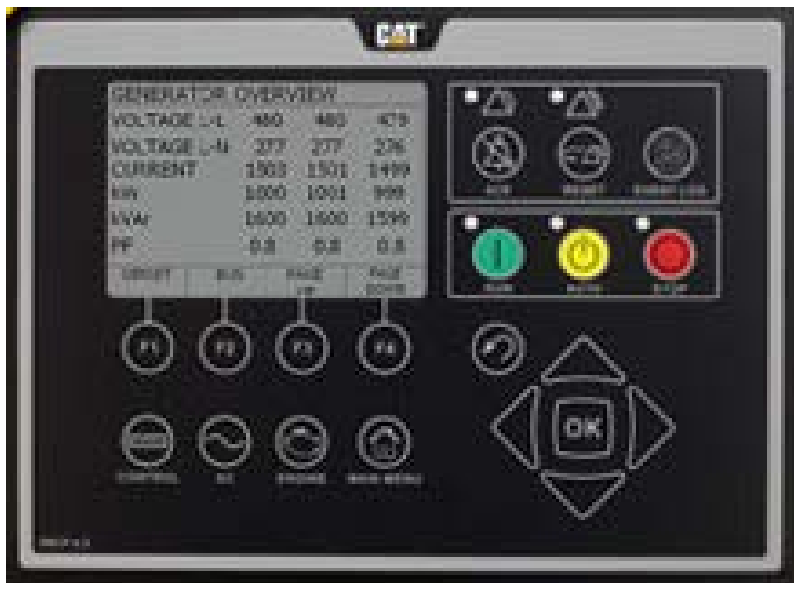

Figure A-4. The Human-to-Machine Interface (HMI), or Graphical User Interface (GUI), of the SAGE EMCP 4.4 Generator Controller 


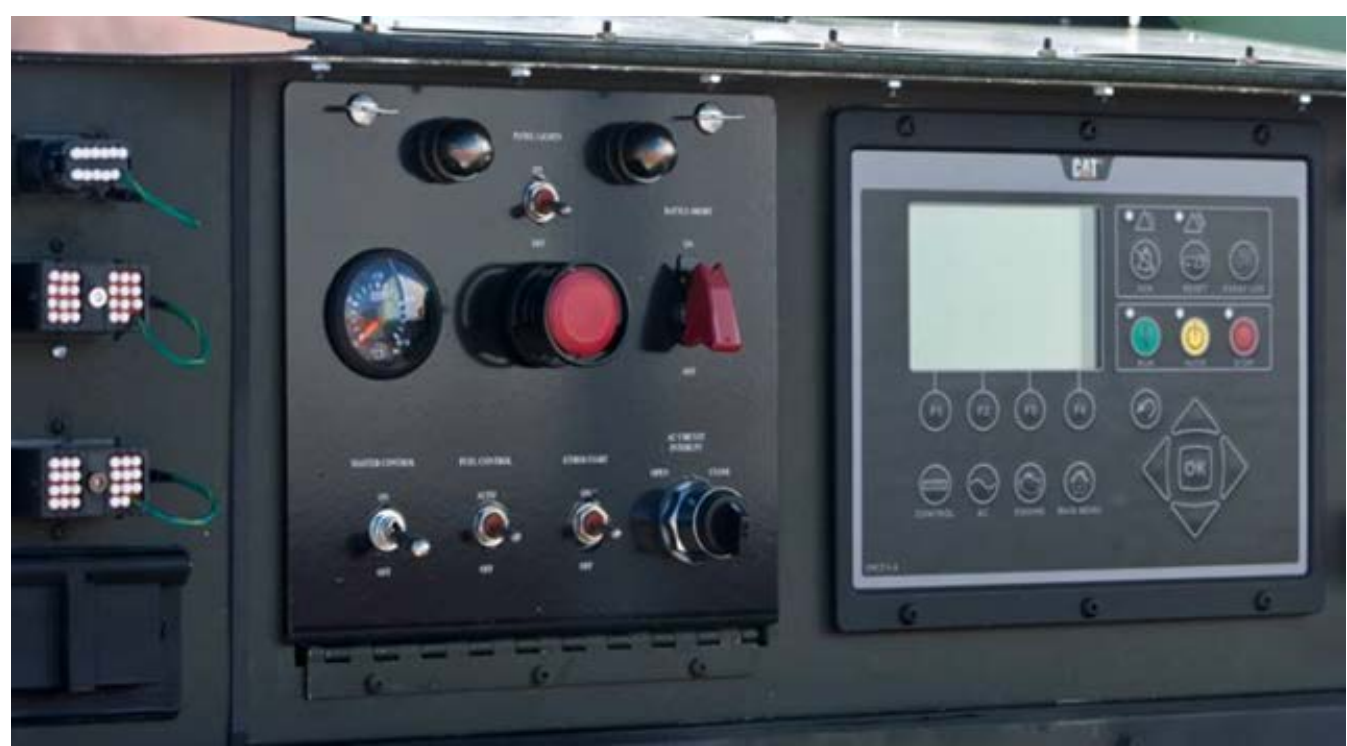

Figure A-5. The EMCP 4.4 as Retrofitted into the Army TQG Generator Used in the SAGE Work at BCIL

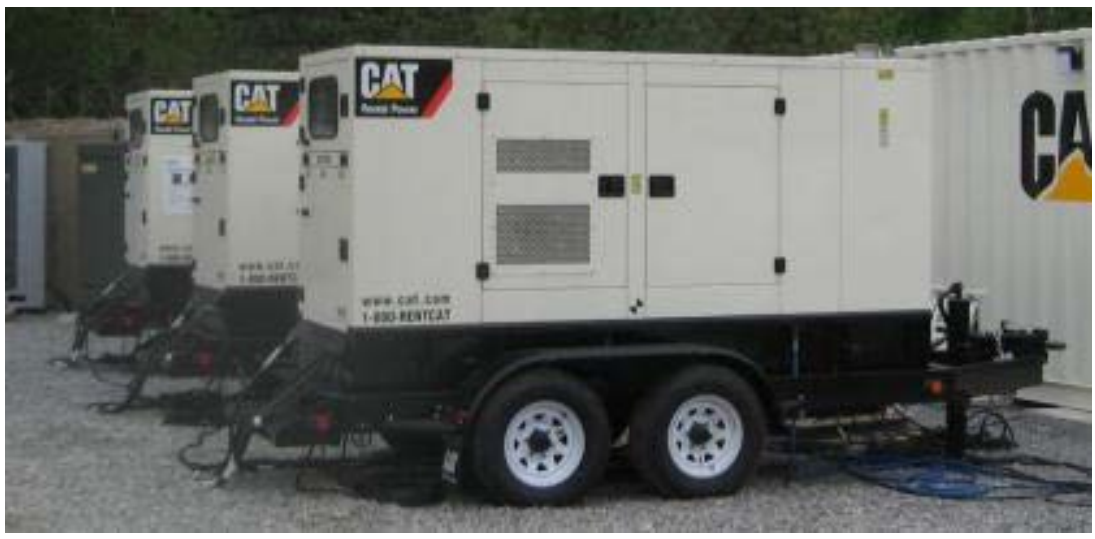

Figure A-6. The Three SAGE Caterpillar Generators 


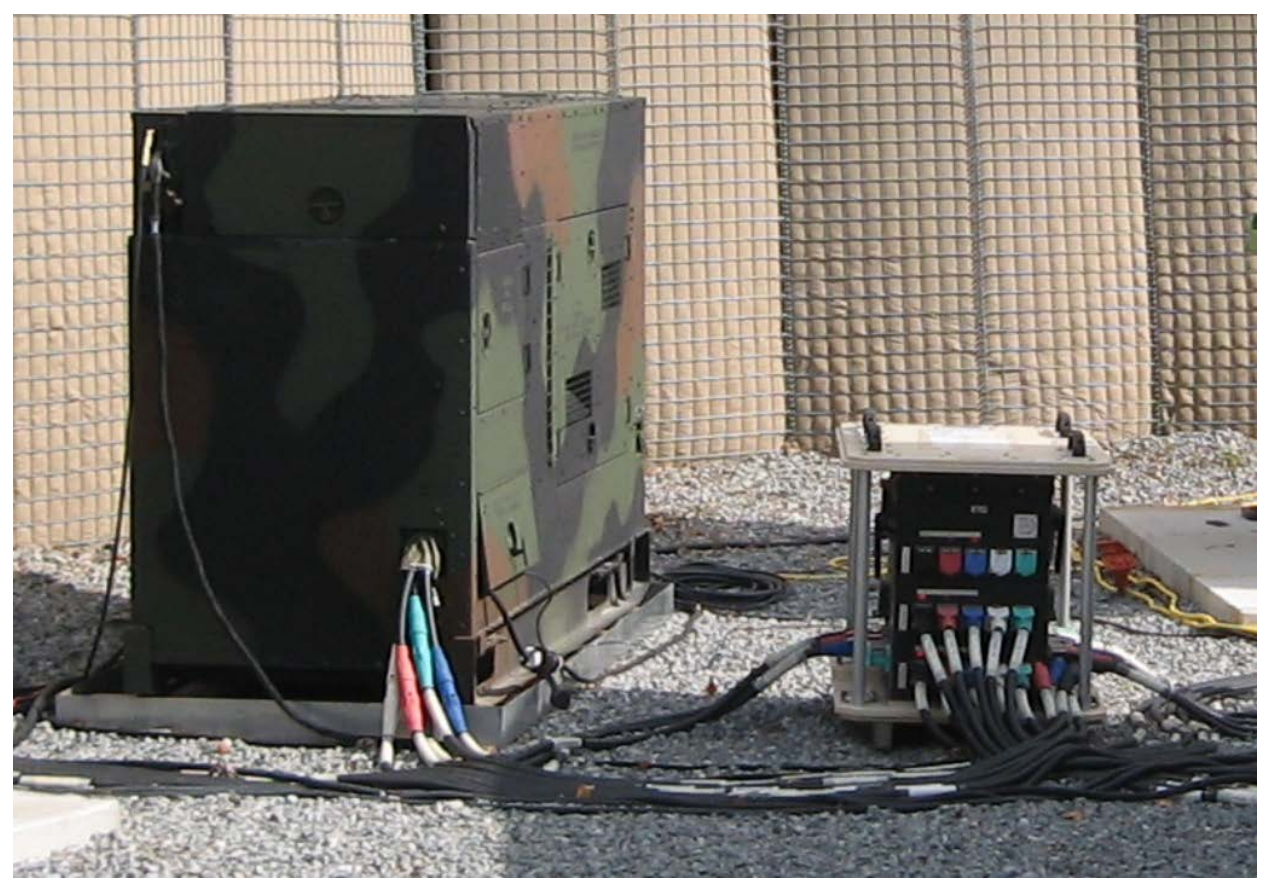

Figure A-7. Left: the TQG that the Army Provided and Caterpillar Retrofitted with an EMCP 4.4 Generator Controller. Right: One of SAGE's Lex Power Distribution Boxes.

\section{SAGE Electricity Storage Unit}

\section{A.1.10 Description}

The SAGE electricity storage unit provides immediately available additional power, or "spinning reserve." The storage works in tandem with the parallel bus generator set to produce power while maintaining the generators in a peak performance range. The storage can actively provide power to the bus to avoid another generator coming online while demand is increasing, or it can use the generators to charge the battery bank as load decreases. Keeping the generators running in their peak performance operating range is part of a fuel saving strategy for the smart microgrid. A storage system also improves the reliability of power and the cleanliness of power in terms of voltage and frequency stability. Storage also supports renewable energy sources such as PV and wind power, and it provides tactical backup power for unplanned outages.

The SAGE demonstration focused on the battery type of electricity storage but other storage systems exist, such as flywheels, and they may also be appropriate for contingency bases.

The key storage technology for electrical types of storage is the power inverter that manages the constantly changing balance of power loads against power supply. 


\section{A.1.11 Specifications}

\section{A.1.11.1 Bidirectional Inverter}

- Provide a bidirectional inverter (BDI) to control the flow of power to and from the storage, and to convert DC power from the batteries into conditioned, clean AC power to the microgrid for a given specified duration.

- Convert AC power from the microgrid (whether the power sources are generators or renewables) to DC power to recharge the battery storage.

- Use a field-proven COTS BDI. Include a cooling system for waste heat of the inverter power converters and controls.

- Consider providing a BDI that can work with several types of batteries.

\section{A.1.11.2 Batteries}

- Provide electricity batteries to store power. The battery type can be one or more of any battery type, including nickel-metal hydride (NImH), lithium-ion, lead-acid, or zinc-bromide flow batteries. Provide a BDI and battery management and control system appropriate for the chosen battery selection.

- Provide battery types that are COTS from the perspectives of the military unit managing the storage and the host country so that replacement batteries are easily available, that local personnel can better understand the technology, and so that batteries that are removed from the unit can be sold for local reuse or salvage.

\section{A.1.11.3 Controller and Monitor}

- Provide a touch screen, also known as a human-to-machine interface (HMI), mounted into the front of the cabinet enclosure of the BDI and its supporting equipment. Provide a GUI for the touch screen to monitor and control the microgrid system. Monitor and control each generator, renewable energy inputs, power flow $(\mathrm{kW})$ on the bus to or from the generators, battery charge and discharge, generation, and load demand.

- Provide a simplified, interactive GUI diagram of the base camp microgrid equipment. Partition the GUI into distinct visualizations, software windows, or screens that provide different detail and perspectives of base camp microgrid equipment. Organize the GUI screens into a hierarchy such that the amount of displayed detail increases as the user "drills down" into the successive GUIs to obtain more detailed or specific data and reports.

- Provide GUIs showing the following information and controls:

- display real-time performance data from base camp microgrid equipment. Position the data displays near associated graphical depictions of the base camp microgrid 
- indicate conditions, or alarms, using flashing, color-coded icons where the color coding reflects the severity of the alarm

- include information related to the alarm, such as a description of the alarm and any associated field device instruments, facilities, and equipment

- show all active alarms and provide controls to clear, disable, or inhibit one or more alarms

- provide input fields to allow operators to define off-normal conditions as excursions of parameters (field device instrument, performance, utility data) above or below operator-defined limits

- Design the monitoring and controls to enable electrical interconnection of two or more storage units into an integrated system. Some of the units will contain a BDI and batteries, and others will contain only batteries. Design the BDI/battery units to monitor and control any of the units.

- Refer to the SAGE Contingency Base Energy Management System specification for additional requirements and considerations, and for integrating the storage with base operations.

\section{A.1.11.4 Containerization}

- Package the storage system into Tricon containers. Tricons are one-third the length of the standard $20 \mathrm{ft}$ container. Provide a single Tricon that integrates the BDI with as many batteries as the Tricon will accommodate, and a controller/monitor to make a core, standalone storage system. Provide additional Tricons of additional batteries for control by a BDI in another Tricon. Limit the weight of the Tricon and its contents to less than 10,000 pounds so that smaller forklifts can move individual Tricons at a base camp.

\section{A.1.11.5 Placement}

- Consider deploying batteries for critical operations such as command centers and medical facilities.

- Position the batteries as close to the end use as possible to minimize power loss due to line losses.

\section{A.1.12 Scalability}

As base camp sizes increase from the 150-Soldier size, deploy additional battery units based on tactical needs for backup/emergency power. 


\section{A.1.13 References}

\section{A.1.13.1 Unified Facilities Criteria}

- UFC 3-520-05, Stationary Battery Areas

- UFC 3-501-01, Electrical Engineering

- UFC 3-550-01, Exterior Electrical Power Distribution

- UFC 3-560-01, Electrical Safety, O\&M; with Change 4

\section{A.1.13.2 Unified Facilities Guide Specifications}

- UFGS 230923.13 20, BACnet Direct Digital Control Systems for HVAC

- UFGS 2510 10, Utility Monitoring and Control System (UMCS) Front End and Integration

- UFGS 260571.00 40, Low-Voltage Overcurrent Protective Devices

- UFGS 2609 13, Power Monitoring System

- UFGS 2611 14.00 10, Main Electric Supply Station and Substation

- UFGS 2612 19.10, Three-Phase Pad-Mounted Transformers

- UFGS 262714.00 20, Electricity Metering

- UFGS 263623.00 20, Automatic Transfer Switches

- UFGS 264100.00 20, Lightning Protection System

- UFGS 283113.00 40, Fire Detection and Alarm Control, GUI and Logic Systems

\section{A.1.14 Incorporate SAGE Technologies into Specifications}

- Revise the existing specifications, or create new sections, for power storage using the specifications.

- The proprietary power storage demonstrated during SAGE field tests may be useful to include as an "or equal" reference in the appropriate specifications, such as TCMS TOGS and ZZ-NSNs, for the purpose of defining the quality and the range of functions. The SAGE storage was assembled by Caterpillar from COTS products. The storage was successfully demonstrated in the SAGE demonstration, meeting the SAGE requirements. Write the specification revisions or new sections to focus on the nonproprietary requirements and then include the statement "Provide a Caterpillar BDI-250 electrical inverter controller or equal" as the key and relatively unique component. 


\section{A.1.15 Key Equipment Deployed in the SAGE Demonstration at BCIL}

\section{A.1.15.1 Inverter}

The inverter was a Caterpillar BDI-250 $250 \mathrm{~kW}$ BDI previously developed for Caterpillar's all-electric-drive tractor line and then adapted for the SAGE demonstration as a new product line. The inverter system included a cooling system for the inverter power electronics.

\section{A.1.15.2 Batteries}

The power storage was four sets or banks of 384 rechargeable, vented lead-acid batteries known as GNB ${ }^{\circledR}$ Absolyte ${ }^{\circledR}$ absorbed glass mat (AGM) batteries. Jumpers interconnected the banks into series. This storage produced two hours of run time at $100 \mathrm{~kW}$ loading.

\section{A.1.15.3 Monitoring and Control}

The controls were a GUI touch screen mounted on the front of the BDI cabinet. The GUI provided monitoring and control of the entire Caterpillar microgrid system. The GUI controlled three operations of the microgrid: 1) run generators only, 2) run generators and battery, and 3 ) run on battery only (also referred to as the "silent run" mode). The GUI also provided monitoring of each generator, renewable energy input, power flow $(\mathrm{kW})$ on the bus to or from the generators, battery charge and discharge, generation and load demand.

\section{A.1.15.4 Containerization}

The container was a $20 \mathrm{ft}$ International Organization for Standardization (ISO) shipping container with standard swing doors at one end, and a man door on one side. The container and its three-axle undercarriage/trailer weighed 55,000 pounds. An air conditioning unit removed excess heat from the batteries' charging/recharging system.

\section{A.1.15.5 Contacts}

Caterpillar provided all of the above equipment as an integrated system delivered to BCIL. The Caterpillar contacts are Jerry Datzman, 309-578-5593, and Barry Payne, 309-578-8447. 


\section{A.1.16 Photos of the Deployed SAGE Systems at BCIL}

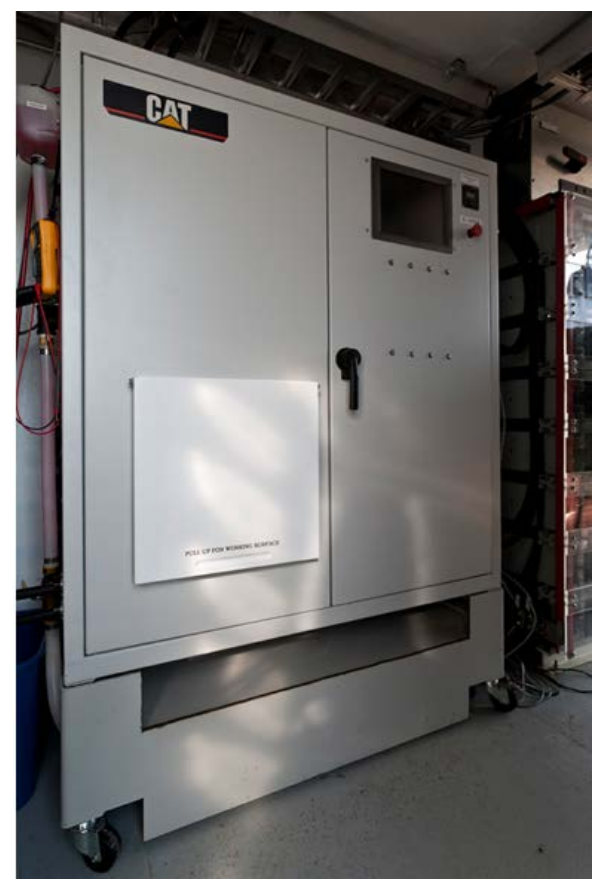

Figure A-8. The BDI-250 Inverter Cabinet as Installed in the Caterpillar Battery Container for SAGE at BCIL

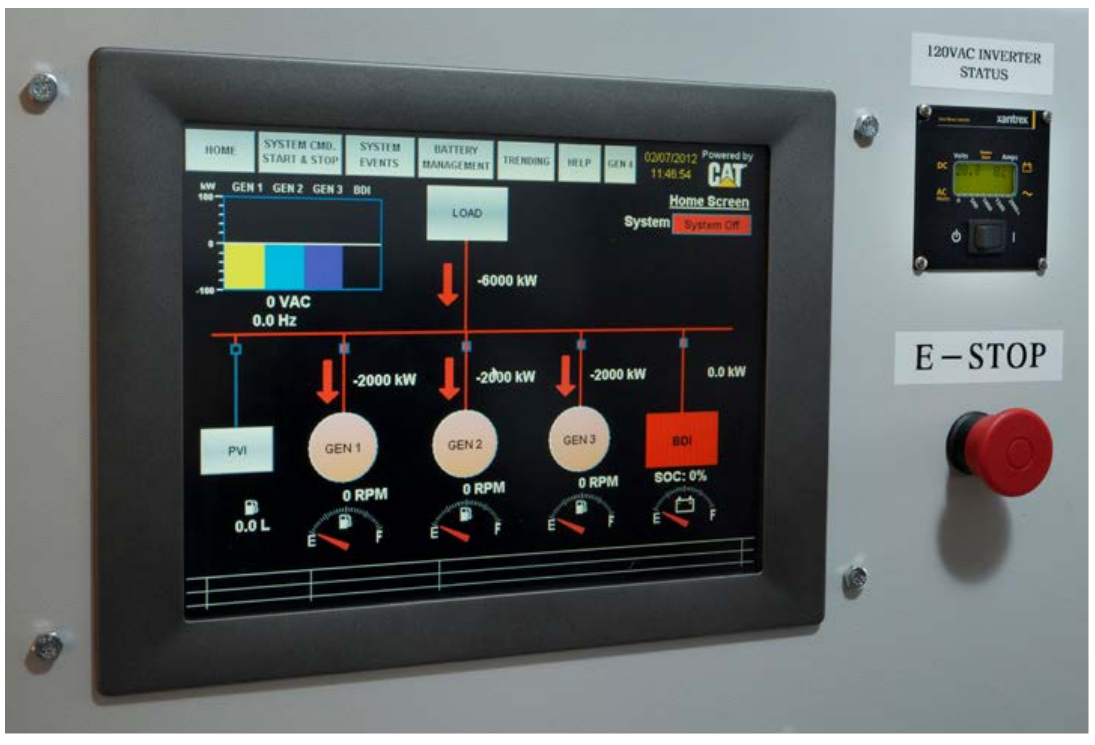

Figure A-9. The HMI or GUI on the Face of the BDI-250 Cabinet 


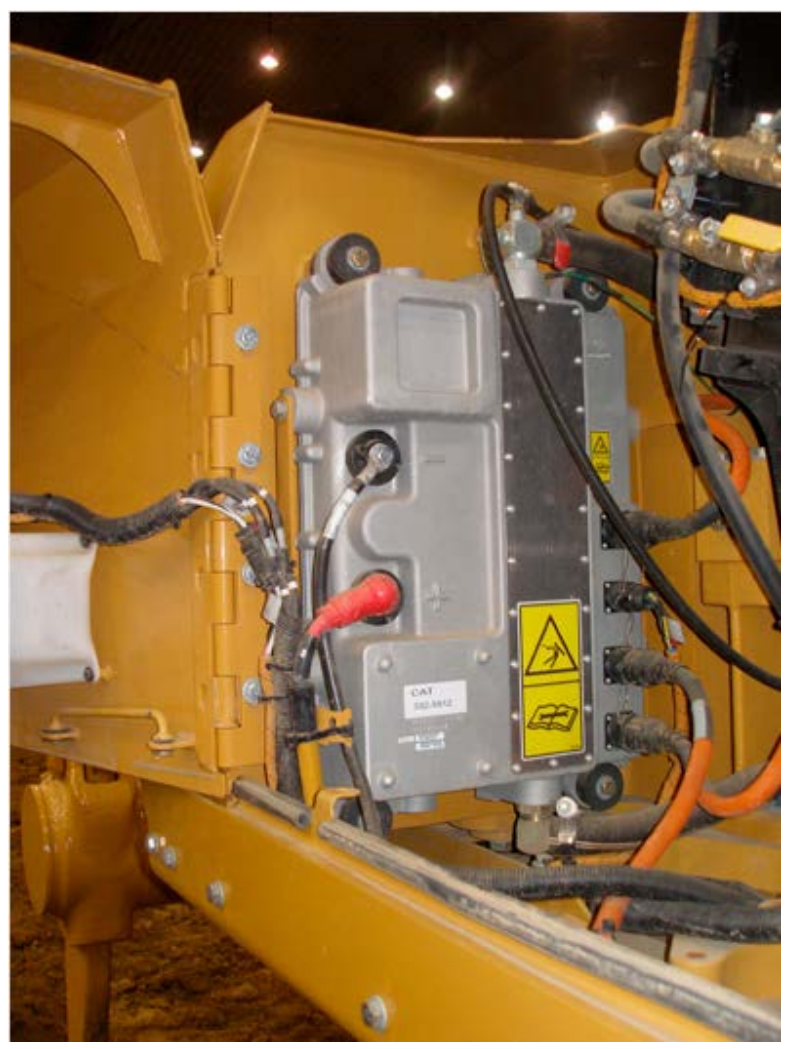

Figure A-10. The Commercial Inverter Developed for Caterpillar's D7 Electrically Driven Tractor Line and Shown as Installed in a Tractor

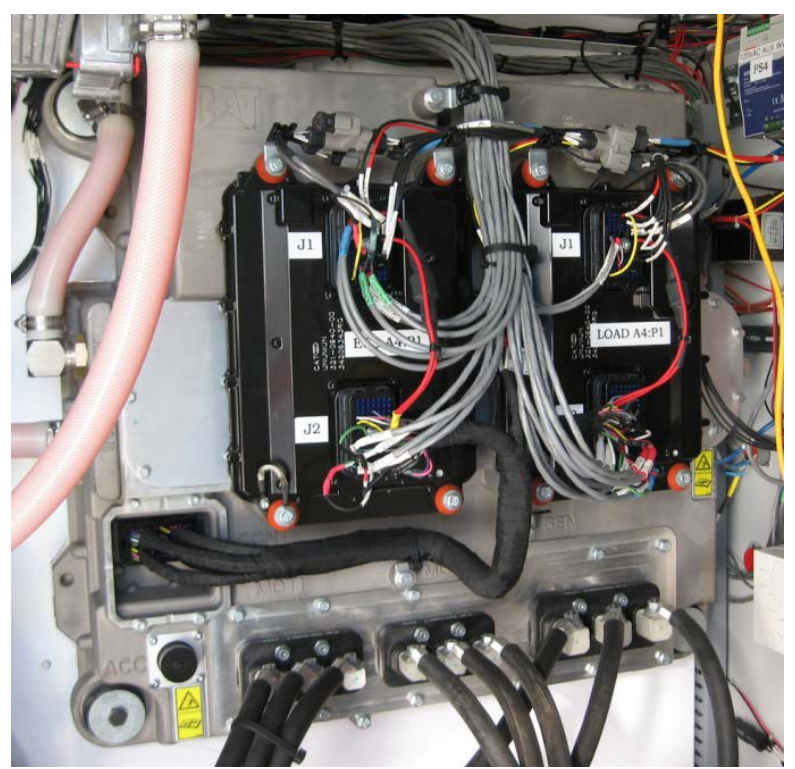

Figure A-11. The D7 Inverter as Installed Inside the BDI-250 Cabinet 


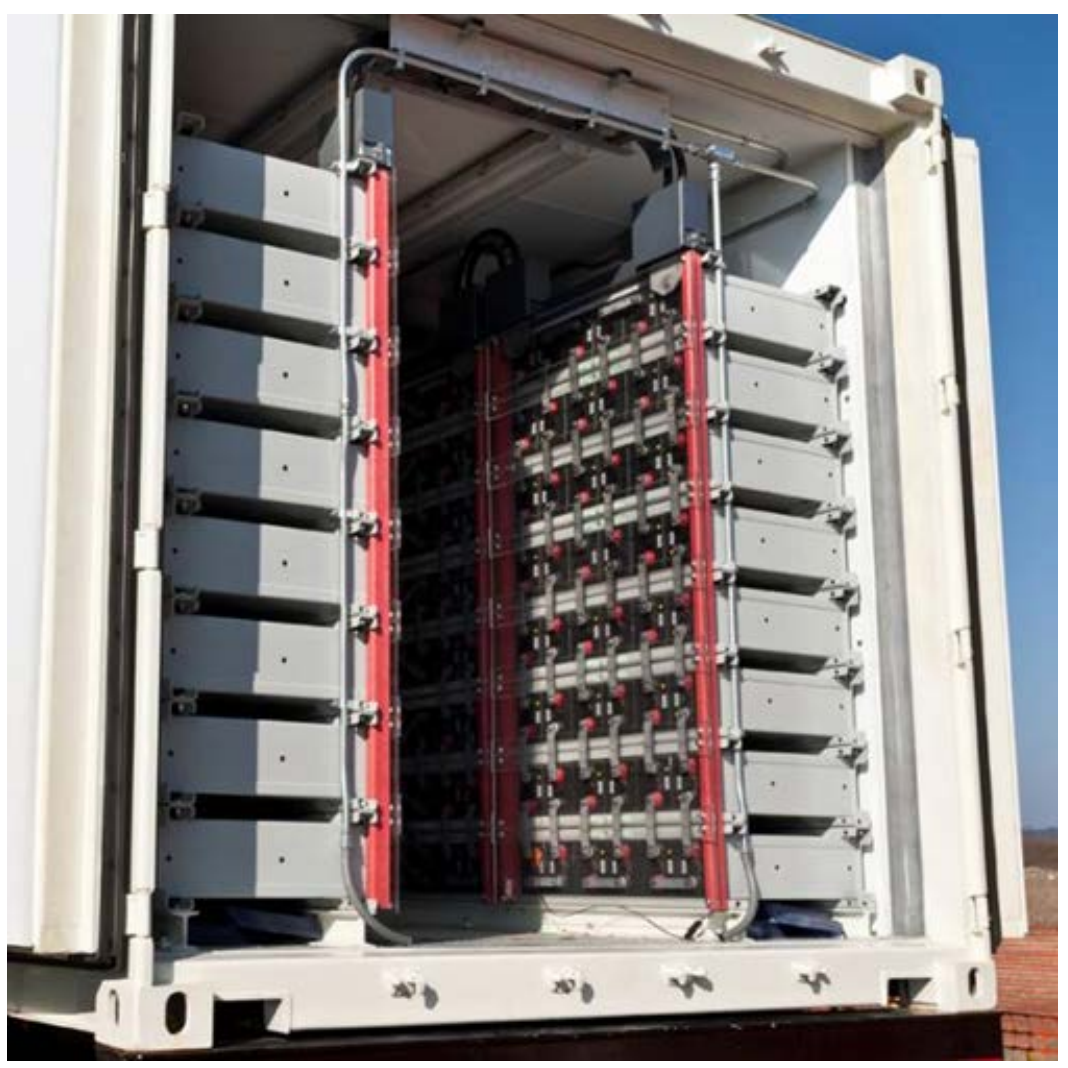

Figure A-12. The Batteries in Racks in the Caterpillar Energy Storage Container

\section{Solar Photovoltaic Power}

\section{A.1.17 Description}

The SAGE PV system converts sunlight into electricity using COTS PV panels to make DC power. Microinverters convert the DC to into safer and more grid-compatible AC power.

The key PV technologies are these PV panels and microinverters. For PV systems, PV panels are assembled into "arrays" of panels.

\section{A.1.18 Specifications}

A wide variety of COTS PV panels and inverters are available. Select the products appropriate for your targeted project.

\section{A.1.18.1 Solar PV Panels}

- Provide panels to meet the project's desired maximum continuous power, maximum input voltage, and maximum input current. Provide panels that meet the following requirements:

- provide $15 \%$ or higher efficiency

- provide a warranty of at least 25 years at $80 \%$ output 
- provide bypass diodes for protection

- operating module is designed for a temperature range of -40 to $85^{\circ} \mathrm{C}$ or better

- provide a maximum series fuse rating of $15 \mathrm{amps}$

- provide nominal 24 volts

- limit reverse current to 15 amps maximum

- design to a maximum system voltage of 600 volts

- design to a power output tolerance of 0 to +4.99 watts

- provide equipment with Underwriters Laboratories (UL) 1703 and/or IEC 61730 labels

- provide equipment and parts manufactured by an ISO 9001 or higher certified factory

- use dust-tight and outdoor weather-resistant electrical connections

\section{A.1.18.2 Microinverter}

- Consider using microinverters to convert the PV DC power into AC power, both for improved safety and for more direct compatibility with the electrical grid. Provide microinverter products that meet the following requirements:

- provide a nominal output voltage of $208 \mathrm{VAC}$, three-phase wye service and five wire (line V1, line V2, line V3, neutral and ground)

- provide nominal output frequency of $60 \mathrm{~Hz}$

- provide efficiencies greater than 96\% per the California Energy Commission efficiency program

- provide a system design to perform an ambient temperature range of $-40^{\circ} \mathrm{C}$ to $65^{\circ} \mathrm{C}$

- provide outdoor rated equipment conforming to National Electrical Manufacturers Association (NEMA) 4 or better

- provide certification per UL 1741

- provide overload relays

- provide monitoring of the three-phase voltage and detecting of the loss of phase

- provide a system design that opens all three phases of circuit when power flow is disrupted

- limit the AC line voltage rise on microinverters to $2 \%$ or less 


\section{A.1.18.3 Monitoring and Controls}

- Provide the following solar panel and system monitoring functions:

- voltage (L-L, L-N) AC side and (voltage) DC panel side

- current (per phase), AC side and DC side

- average volts, amps, and frequency

- $\mathrm{kW}$ and $\mathrm{kVA}$ (average, phase, percent) power factor (average, phase)

- energy $\mathrm{kWh}$ and $\mathrm{kVAr}-\mathrm{h}$ (total)

- generator stator and bearing conditions

- onboard system diagnostics

- front-panel keyboard access to monitoring and controls

- computer interface for PV performance and monitoring

- consider providing web-based monitoring

- If using microinverters, then provide an open communication system using one or more of the following protocols:

- RS-232

- RS-485 annunciator data link

- Modbus TCP, 10BT Ethernet

- Modbus RTU, RS-485 half duplex

- Power Line Carrier

- BACnet.

Refer to the SAGE Contingency Base Energy Management System specification for additional requirements and considerations, and for integrating the PV with base operations.

\section{A.1.18.4 Foundations}

- Provide a means to anchor the PV panels and arrays thereof to the ground or a structure.

- Consider sizing the support structure for the maximum wind loads and snow loads in Theater so that the PV can be relocated to sites without revising the structure. Many types of foundations and related products can be considered, and some may be COTS. 
- Consider using a foundation that can be reused and relocated to other locations in a base camp or to other regions.

- Consider wind-resistant ballasts that use local materials, such as on-site gravel, placed in chain-link type of enclosures.

\section{A.1.18.5 Logistics}

- Design the PV system, including the support for the foundation structure, to fit into Tricons to simplify the position adjustments and the relocation of PV to other sites.

- Consider using quick disconnect type junction boxes.

\section{A.1.18.6 PV Location}

- Consider not placing PV on building roofs because the location is a barrier to PV maintenance and cleaning and a barrier to roof repairs. Alternatively, consider placing PV over occupied structures, such as billets and offices, to provide shade to the buildings in hot seasons; the PV support structure may be directly connected to the roof or may be a free-standing structure.

\section{A.1.19 Scalability}

- As base camp sizes increase from the 150-Soldier size, deploy additional PV arrays based on long-term cost-effectiveness and on tactical needs for backup/emergency power.

- Consider deploying arrays at central locations, such as battalion headquarters, to simplify PV operations and maintenance but still be physically close to the buildings using the PV power.

\section{A.1.20 References}

\section{A.1.20.1 Unified Facilities Criteria}

The UFC does not have specifications addressing PV.

\section{A.1.20.2 Unified Facilities Guide Specifications}

The UFGS does not have specifications dedicated to PV, but the following are related to some of the electrical work required for PV:

- UFGS 2605 71.00 40, Low-Voltage Overcurrent Protective Devices

- UFGS 2609 13, Power Monitoring System. 


\section{A.1.20.3 Solar PV Mounting Systems}

Consider meeting the following standards until your organization's design authority provides design direction:

- Load Bearing and Statistical Data

- International Building Code 2006 (or 2009 where required)

- American Society of Civil Engineers/Structural Engineering Institute (ASCE/SEI) 7-05, Minimum Design Loads for Buildings and Other Structures

- Material Verification

- Aluminum Design Manual 2010

- Steel Construction Manual, 14th Edition

- Solar PV Installation

- UL 1703 listed and/or IEC 61730 qualified

- Current National Electrical Code (NEC) guidelines for cabling, power junctions, voltage rise/drop and cabling

- Microinverter Compliance

- UL 1741/ Institute of Electrical and Electronics Engineers (IEEE) 154, Federal Communications Commission Part 15 Class B

- CAN/Canadian Standards Association (CSA)-C22.2 NO. 0-M91, NO. 4-04, and NO. 107.1-01

\section{A.1.21 Incorporate SAGE Technologies into Specifications}

- Create new specification sections to incorporate the PV specifications.

- The proprietary PV products demonstrated during SAGE field tests may be useful to include as an "or equal" reference in the specifications, including TCMS TOGS and ZZ-NSNs, for the purpose of defining the quality and the range of functions. The SAGE PV panel was a PVM220PS-230 (230 watt) panel provided by the Federal Department of Justice Federal Prison Industries (also known by the trade name of UNICOR). The microinverter was an Enphase M215-60-2LL-23 microinverter. The PV system was successfully demonstrated in the SAGE demonstration, working as specified. Write the specification revisions or new sections to focus on the nonproprietary requirements and then include the statement "Provide Federal Prison Industries, PVM220PS-230 (230 watt) solar modules and Enphase M215-602LL-23 microinverter, or equals." 


\section{A.1.22 Key Equipment Deployed in the SAGE Demonstration at BCIL}

\section{A.1.22.1 PV Panels}

UNICOR selected PV solar panels Model UNICOR PVM220PS-230. Each panel can produce up to 230 watts. SAGE used 60 panels divided into two arrays of 30 panels each. Arrays were set at a fixed angle of 42.5 degrees. Each array was approximately $14 \mathrm{ft} \times 44 \mathrm{ft}$ and produced up to $6.45 \mathrm{~kW}$ with three-phase $208 \mathrm{VAC}$ power.

\section{A.1.22.2 Monitoring and Control}

The PV system was connected to the SAGE/BCIL network to monitor the power generation and condition of the system. The communication equipment was located next to the solar arrays and enclosed in a NEMA 4 enclosure for weather protection.

Detailed PV data was obtained from the microinverter company's website.

See Figure A-13, "Solar Array with Microinverter Generation for a 150-Soldier Base Camp," for layout info.

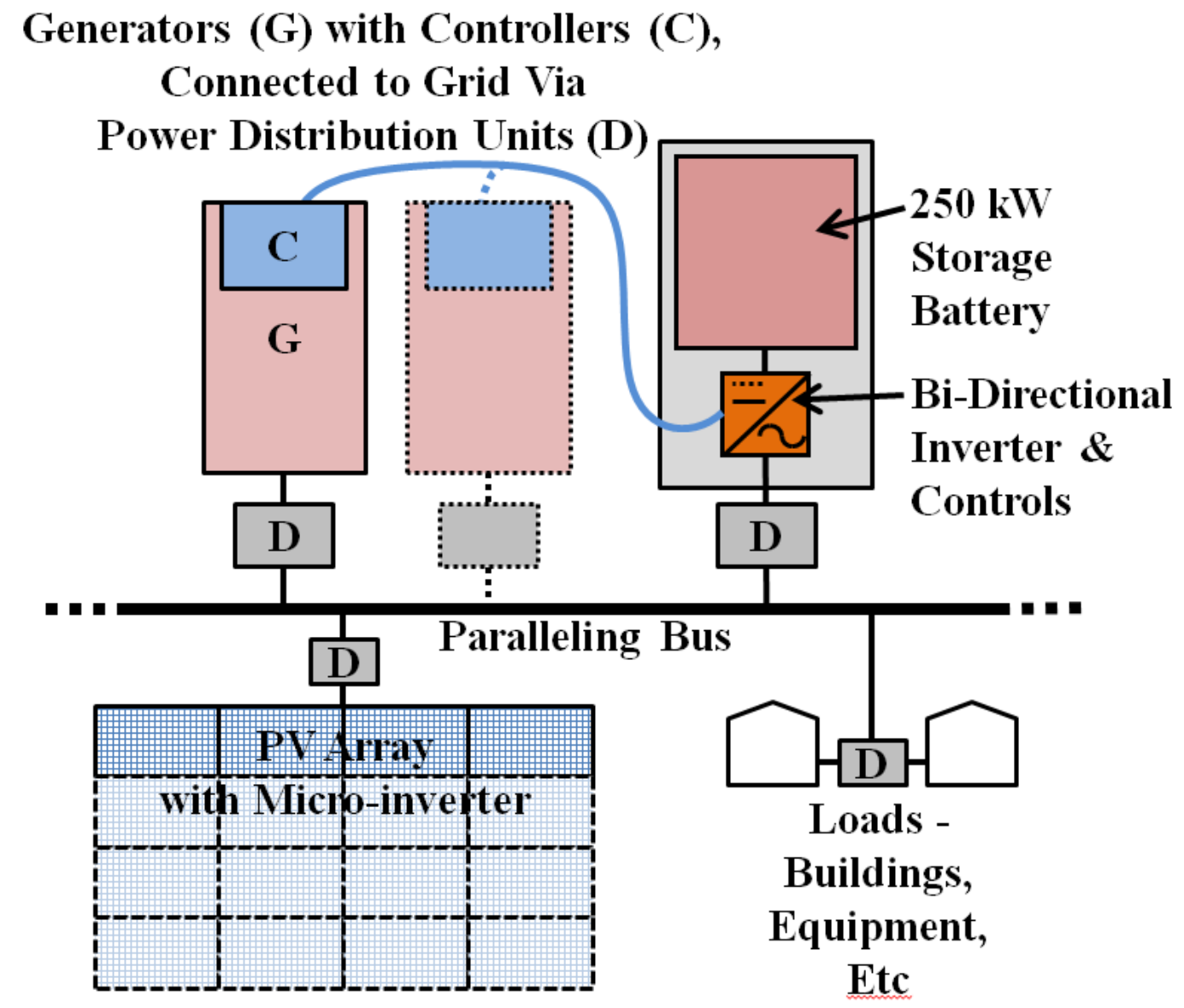

Figure A-13. Solar Array with Microinverter Generation for a 150-Soldier Base Camp 


\section{A.1.22.3 Microinverters}

One microinverter, an Enphase Model M215-60-2LL-S23, was installed on each panel. SAGE connected the microinverter to the BCIL network to enable the communications link and data transfer that is part of the Enphase web-based service of data display and diagnostics. Screen shots of two of the available Enphase web screens are shown in Figure A-17 and Figure A-18. Enphase's screens were also available through SAGE's Contingency Base Energy Management System (CB-EMS).

\section{A.1.22.4 Electrical System}

The two array outputs were combined into a central load panel that consisted of a $15 \mathrm{amp}$ three-phase circuit breaker for each array. A circuit breaker was used to cut power for servicing the system. The load panel was connected to the generation distribution bus using a portable power cable.

\section{A.1.22.5 Foundation}

The foundation ballast blocks were locally made to meet UNICOR's specifications. UNICOR selected an aluminum mounting structure by Mounting Systems GmbH designed especially to support PV arrays; additional information is at http://ww3.mountingsystems.info/en/open-terrain-mounting-system-sigma2.html. UNICOR planned the nextgeneration foundation to be $\mathrm{Hesco}^{\circledR}$ wall-type bins holding dirt or rock as the ballast against wind uplift.

\section{A.1.22.6 Contacts}

UNICOR provided the complete PV system, including microinverters and supporting structure. UNICOR's point of contact was Kimberly Schwinn, 202-305-3730.

\section{A.1.23 Photos of the Deployed Equipment}

The following photos show the major components of the PV system as used for SAGE at BCIL. 


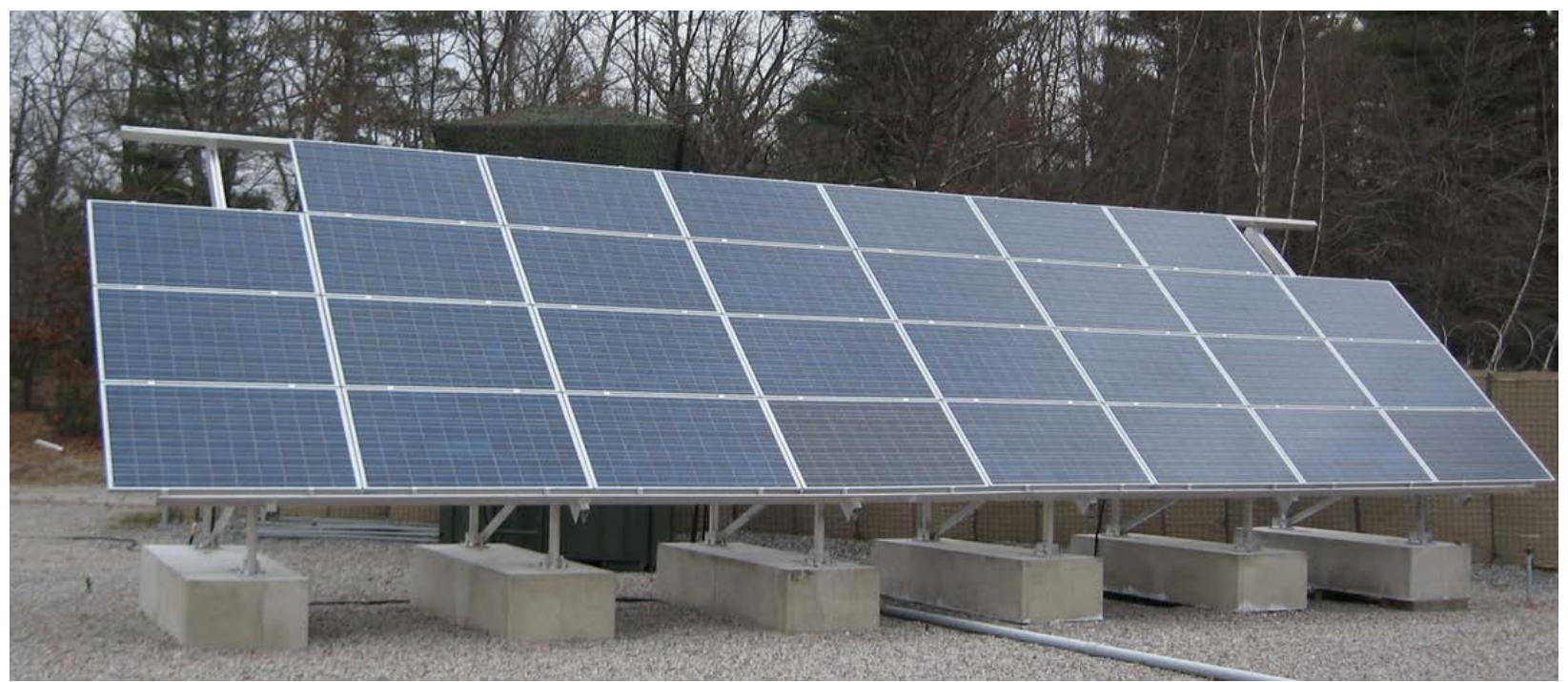

Figure A-14. One of the Two Solar Panel Arrays

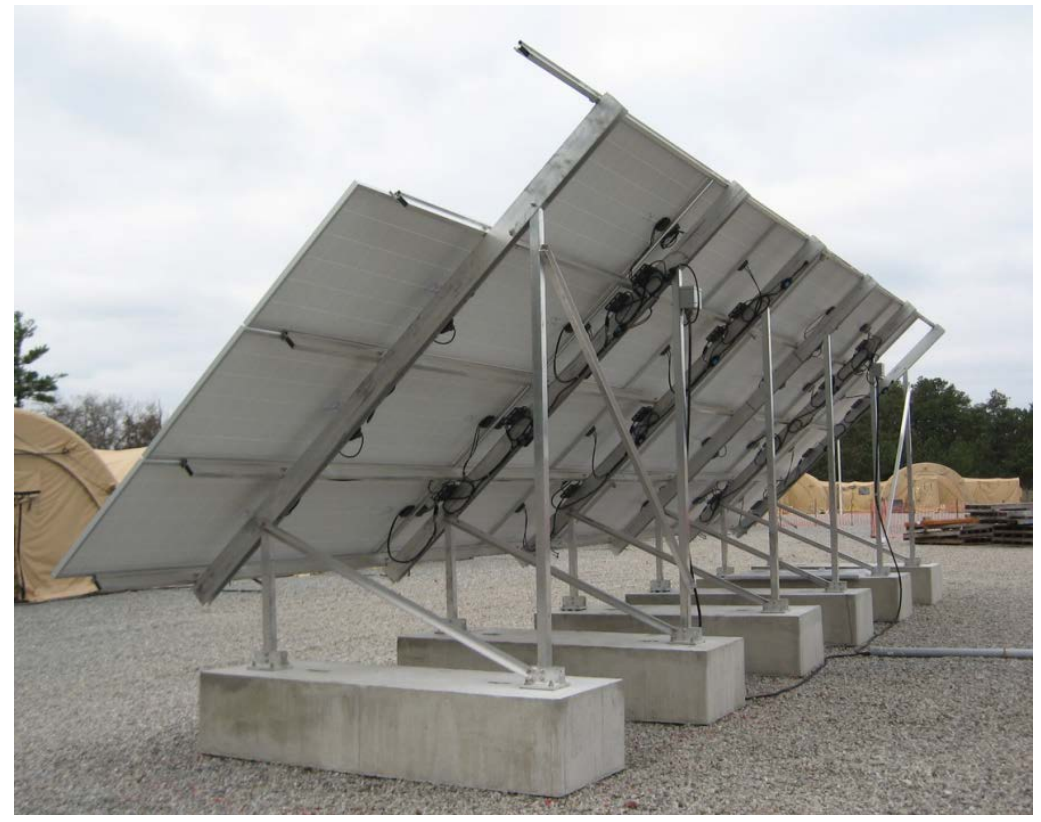

Figure A-15. The Aluminum Frame Support Structure of One Array 


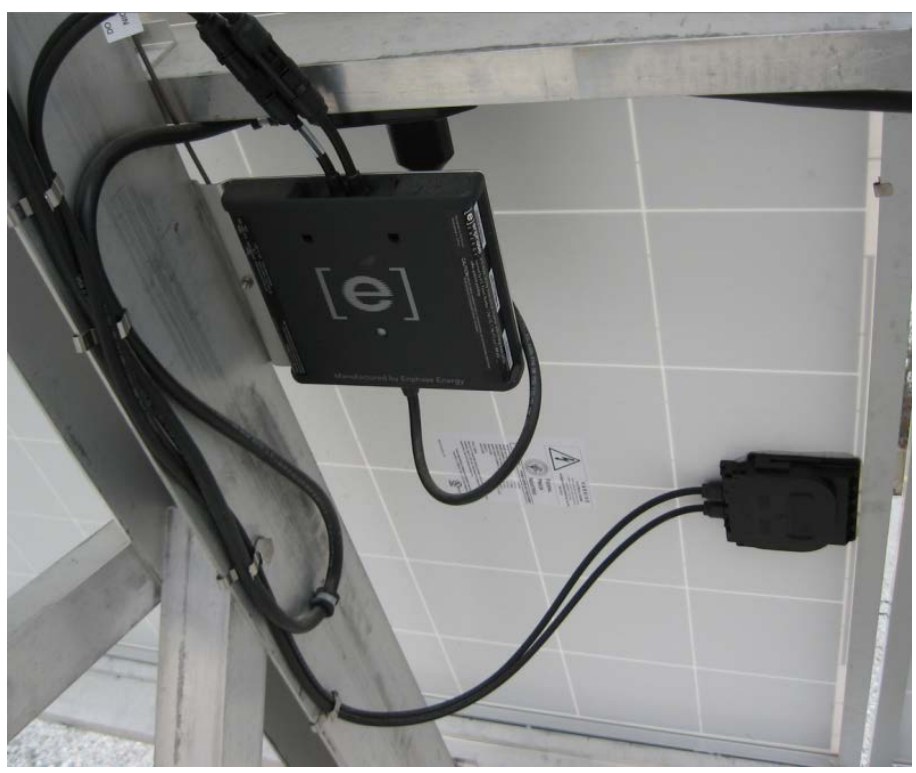

Figure A-16. One of the 60 Microinverters

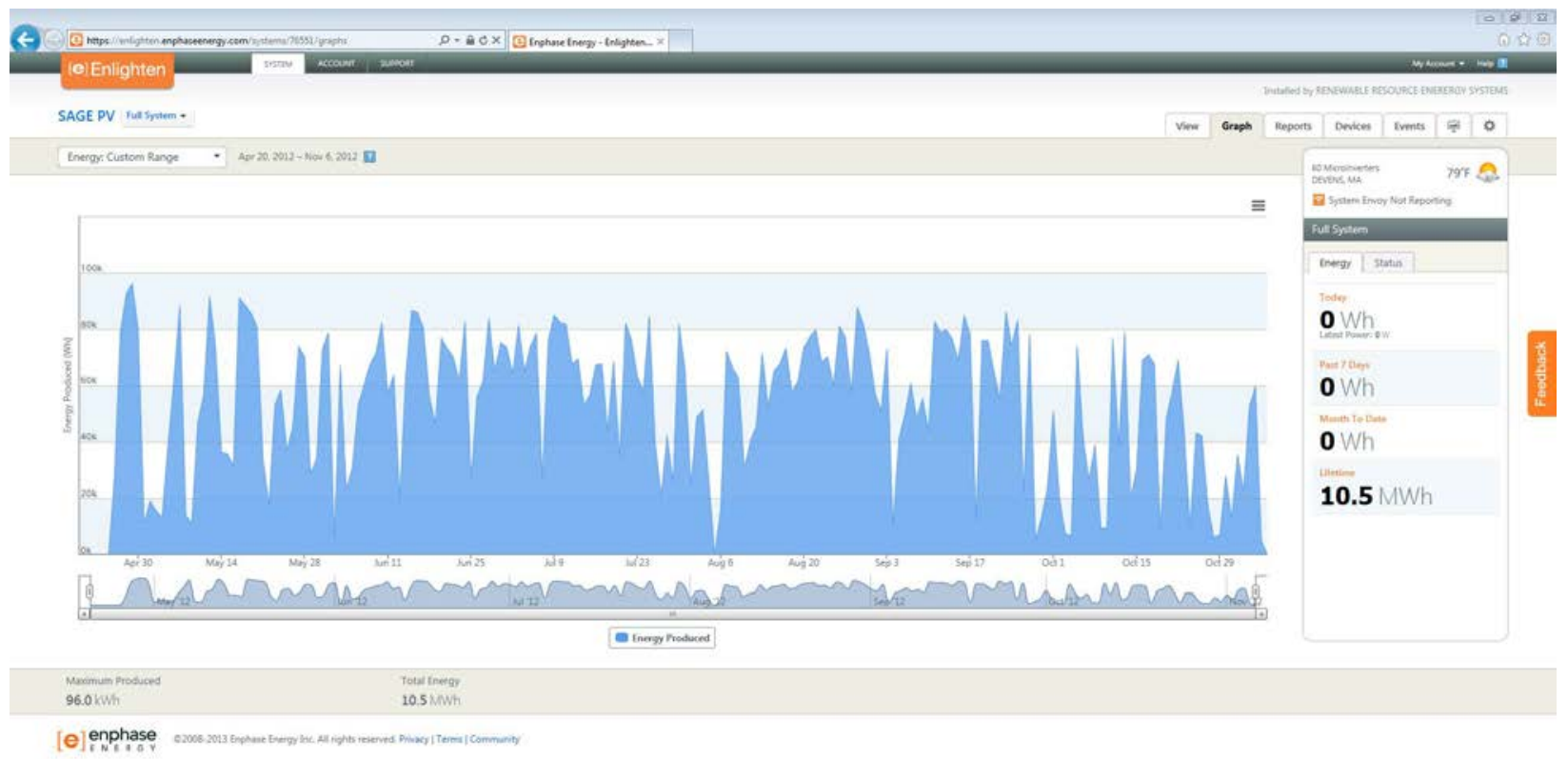

Figure A-17. Enphase's Enlighten Webpage to Access SAGE PV System Data and Performance Information. This screen shows the actual power generated from April 20 to November 5, 2012, which was the core period of the SAGE testing work. 


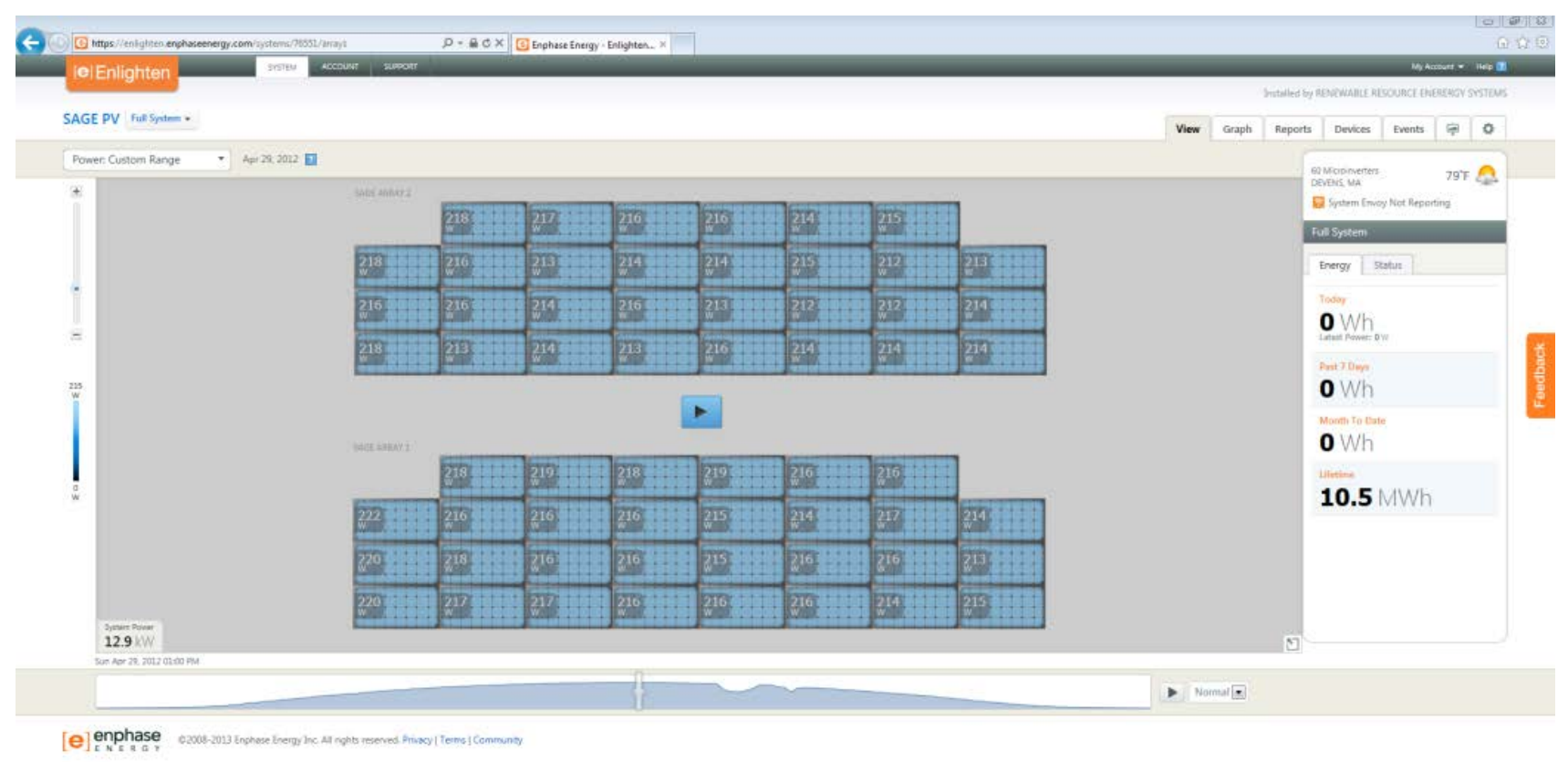

Figure A-18. Enphase's Screen Showing the Peak Output that Occurred on April 28, 2012

\section{Solar Hot Water System}

\section{A.1.24 Description}

The SAGE solar hot water (SHW) system supplements conventional water heating systems to conserve energy.

The key equipment consists of solar collector panels, a storage tank for the solar-warmed water to maximize the SHW system performance, and a freeze protection method.

\section{A.1.25 Specifications}

- Provide a turnkey, integrated, and containerized SHW system to withstand the environmental conditions of the selected site(s) and/or climate zone(s), using COTS products.

- Consider preassembling as much of the SHW system as logistically possible to simplify shipping, assembly, and relocation of the SHW system. Consider having collector array structural frames manufactured with the arrays such that they can be transported in the same shipment packaging and easily installed at the selected site(s) using a small forklift.

- Provide solar collectors that are certified to meet ASHRAE Standard 93-1986 by a nationally recognized laboratory testing organization such the Solar Rating \& Certification Corporation or the Florida Solar Energy Center. Select a collector with a warranty of at least 10 years. Consider selecting higher-efficiency models. 
- Consider selecting a SHW system designed to minimize the electrical consumption of the parasitic power needed for pumping, controls and lighting equipment. Consider using $2 \mathrm{kWh}$ per day as the maximum average parasitic power load.

- Select a SHW system designed to protect against freezing for sites that may be exposed to freezing conditions at some point throughout the year. Consider selecting a drain-back SHW system that avoids freezing by using the force of gravity to drain the collector water to a storage tank when the pumps are off; this drain-back method allows the heat exchange fluid to be untreated water, which reduces on-site maintenance and environmental protection measures. Consider using a freeze protection method that works at all sites, including those that do not need freeze protection, so that the SHW system can be freely relocated.

\section{A.1.25.1 Storage Tank}

- Consider including a storage tank so that the SHW system can create warm water even when the end-use load is low. Size the tank to accommodate the water in the collectors and piping when the SHW system drains back into the tank.

- Provide a tank warranty of at least five years.

\section{A.1.25.2 Heat Exchanger}

- Consider using a heat exchanger to protect the end-use potable water from the collector heat transfer fluid, even when the collector fluid is untreated potable water. Consider selecting a heat exchanger that is submerged in the storage tank to reduce the system footprint and to reduce heat loss to the environment. When including a heat exchanger, use materials that are highly heat-conductive and highly corrosion resistant, and provide as large of a heat transfer surface area as possible.

\section{A.1.25.3 Controls}

- Provide automation so that the system will operate independently and automatically during all four seasons. Include GUIs in the automation to provide the following control, monitoring, and diagnostic information:

- total energy output of the system; temperature of the incoming (source) water supply, volume of the incoming (source) water supply to the system, and temperature of the outgoing water to the conventional water heater

- temperature of the solar hot water tank

- temperatures of the solar collector's water inlet(s) and outlet(s), and interior surface temperature of the collector

- total electrical consumption of the SHW system per time periods selected by users

- state of health of the system (including operation, maintenance and communication status) 
- Control the primary collector loop by a differential temperature controller and include all required flow meters, valves, sensors, and control wiring to achieve a reliable and accurate system.

Refer to the SAGE Contingency Base Energy Management System specification for additional requirements and considerations, and for integrating the SHW system with base operations.

\section{A.1.25.4 Logistics}

- Orient the collectors toward the equator.

- Tilt the collectors at an angle equal to the latitude of the selected site(s) and/or to the manufacturer's recommendations. Position the deployed SHW system to minimize the shade crossing the collectors.

- Design the SHW system to operate normally when the site's grade is not level; allow for up to 6" difference in grade between any two corners of the unit.

\section{A.1.25.5 Containerization}

- Provide the SHW system to be integrated into standard ISO shipping containers for the purposes of shipping and on-site setups. Consider using Tricons, which are one-third the length of a standard $20 \mathrm{ft}$ container, for small sites that have only smaller, lighter-load capacity forklifts for handling containers.

- Provide a single container to mount the collectors and permanently house the support equipment, including pumps, controls, and storage tank. Provide additional containers as necessary to ship the collectors and/or collector to provide support structures.

- Design the SHW system support structure to use the container for wind and seismic resistance and for foundation.

- When Tricons are used, limit the weight of a Tricon and its contents to less than 10,000 pounds so the SHW system container can be moved by smaller forklifts that may be available at smaller sites.

- Provide weather-tight and pest-resistant water line connections through the wall of the container.

\section{A.1.26 Scalability}

- As base camp sizes increase from the 150-Soldier size, deploy additional SHW system based on long-term cost-effectiveness as determined for your particular application. Consider designing larger scale SHW system units that can be containerized. 


\section{A.1.27 References}

\section{A.1.27.1 Unified Facilities Criteria}

- UFC 3-440-01, Active Solar Preheat Systems, June 14, 2002

\section{A.1.27.2 Unified Facilities Guide Specifications}

- UFGS-22 3330.00 10, Solar Water Heating Equipment, April, 2008

\section{A.1.28 Incorporate SAGE Technologies into Specifications}

- Revise the existing specifications for solar water heating equipment to include the features described in this specification.

\section{A.1.29 Key Equipment Used in the SAGE Demonstration at BCIL}

An integrated SHW system was provided by Alternate Energy Technologies, LLC, (AET), in partnership with UNICOR as the general contractor. The SAGE SHW system was transported in a $20 \mathrm{ft}$ container with all the interior components preinstalled.

The SAGE SHW system was a drain-back system using potable water as the heat transfer fluid. The system was designed to fit in a standard ISO $20 \mathrm{ft}$ container for transportation and included a rack system to mount the solar thermal collector arrays on the roof of the container. The container was insulated with 4" polyisocyanurate foam in the ceilings and 2"

polyisocyanurate foam on the sides and doors with R-values of 26 on the ceiling and 13 on the sides and doors. The interior of the container is fitted with brackets to allow the collector mounting racks to be securely stowed during shipping. The container also contained high efficiency light-emitting diode (LED) lighting, and a $1500 \mathrm{~W}$ recessed electric space heater for supplemental freeze protection (the water tank was effectively the space heater for the container's interior). Figure A-19 illustrates the deployed SAGE SHW system. 


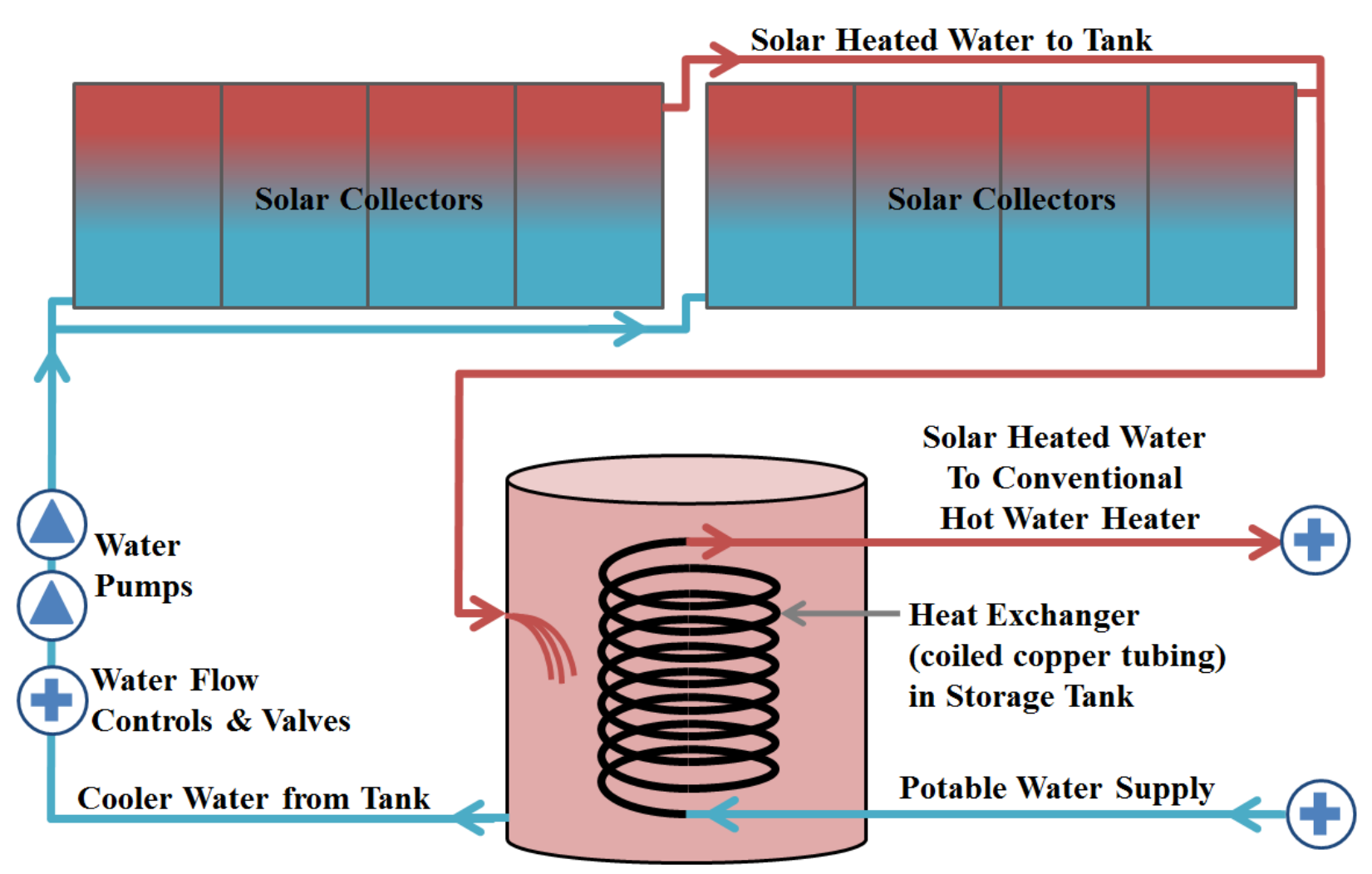

Figure A-19. Solar Hot Water System

\section{A.1.29.1 Solar Array}

The solar thermal collector array consisted of two banks of four $4 \mathrm{ft} \times 8 \mathrm{ft}$ AET Morning Star ${ }^{\mathrm{TM}}$ Model MSC-32 solar thermal collectors, for a total of $32 \mathrm{ft}^{2}$; these are flat-plate glazed collectors that are plumbed (piped) in parallel and designed for uniform flow through each collector. Additional information is at the following link:

http://www.aetsolar.com/literature/MSC_Collector_Brochure.pdf.

Florida Solar Energy Center and Solar Rating and Certification Corporation (SRCC) Summary Information Sheets on MSC-32 Collectors are at the following link: http://mscsolar.com/certifications.php.

\section{A.1.29.2 Controller and Energy Meter}

The controller and energy meter was an Eagle Sun, model \# DTC-ES-1 that included local data transmitting to a personal computer (PC) via RS-232 cabling, and a digital readout that was programmable for setting on/off differentials, storage high limit temperatures, and collector minimums on temperature. 


\section{A.1.29.3 Pumps}

The water pumps were two centrifugal, stainless steel Taco Model \#007FS5, $115 \mathrm{~V}, 60 \mathrm{~Hz}$ single phase, provided with the AET system. These pumps were cartridge style, maintenance free, wet-rotor, in-line, single stage circulator pumps. More information is provided at the following link: http://www.aetsolar.com/pumps-and-controls.php.

\section{A.1.29.4 Fluid Handling System}

The fluid handling system was housed inside the insulated $20 \mathrm{ft}$ ISO container and included the following components, all preinstalled before shipment to the site:

- one 415 gallon solar storage tank insulated with R-14 isocyanurate foam

- three vertical $180 \mathrm{ft}$ Type L soft copper coiled potable-water heat exchangers inside the tank

- storage tank and heat exchanger designed to handle a maximum flow rate of $30 \mathrm{gpm}$. Additional information on similar tanks is at the following link: http://www.aetsolar.com/literature/SystemComponents/Tanks/DBSpecSheet_2013.pdf

- one water pump dedicated to the startup of water flow through the collectors, and designed for a $14.34 \mathrm{ft}$ head requirement at 8 gallons per minute (gpm)

- one water pump dedicated to circulating water after startup, and designed for $5.15 \mathrm{ft}$ head at 15 gpm

- one vortex flow meter with microelectromechanical systems (MEMS) sensors

- an Eagle Sun solar controller

- two output relays to provide variable speed for circulating pump based on temperature differential between tank and collectors as well as fixed speed for startup pump.

\section{A.1.29.5 Monitoring and Telemetry}

Monitoring and remote telemetry was provided with the integrated system by AET. The dataTaker $^{\circledR}$ data logger (model DT80M Series 3) is an intelligent data logger that can provide remote monitoring and automatic data transfer. The logger design provided access via integrated modem, Ethernet and/or Universal Serial Bus (USB) connection. A standard Modbus protocol (RTU) was used for the serial sensor port with File Transfer Protocol (FTP) and Transmission Control Protocol/Internet Protocol (TCP/IP) was also available for USB or Ethernet ports. The integrated modem also provided Wi-Fi Protected Access (WPA2 encryption). Additional information is at the following link:

http://www.dataloggerinc.com/products/DT80M_Intelligent_Universal_Input_Data_Logger/193/

\section{A.1.29.6 Contacts}

UNICOR provided the integrated, containerized SHW system and deployed it on BCIL. UNICOR's subcontractor for the SHW system design, fabrication, and deployment was AET. 
The UNICOR point of contact was Kimberly Schwinn, 202-305-3730, and AET's was John Alger, 800-874-2190.

\section{A.1.30 Photos of the Deployed Equipment}

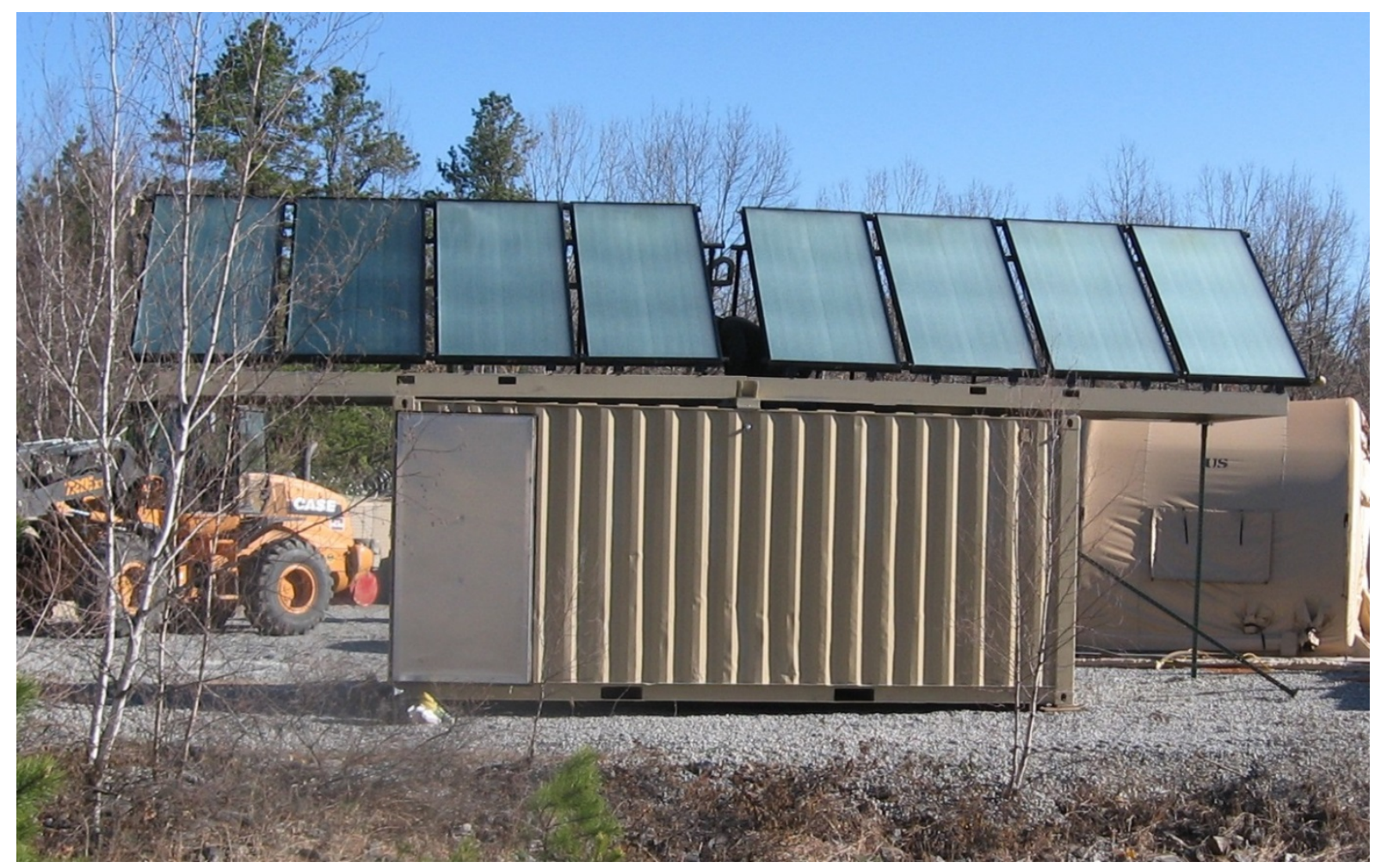

Figure A-20. SAGE SHW System Operational at BCIL 


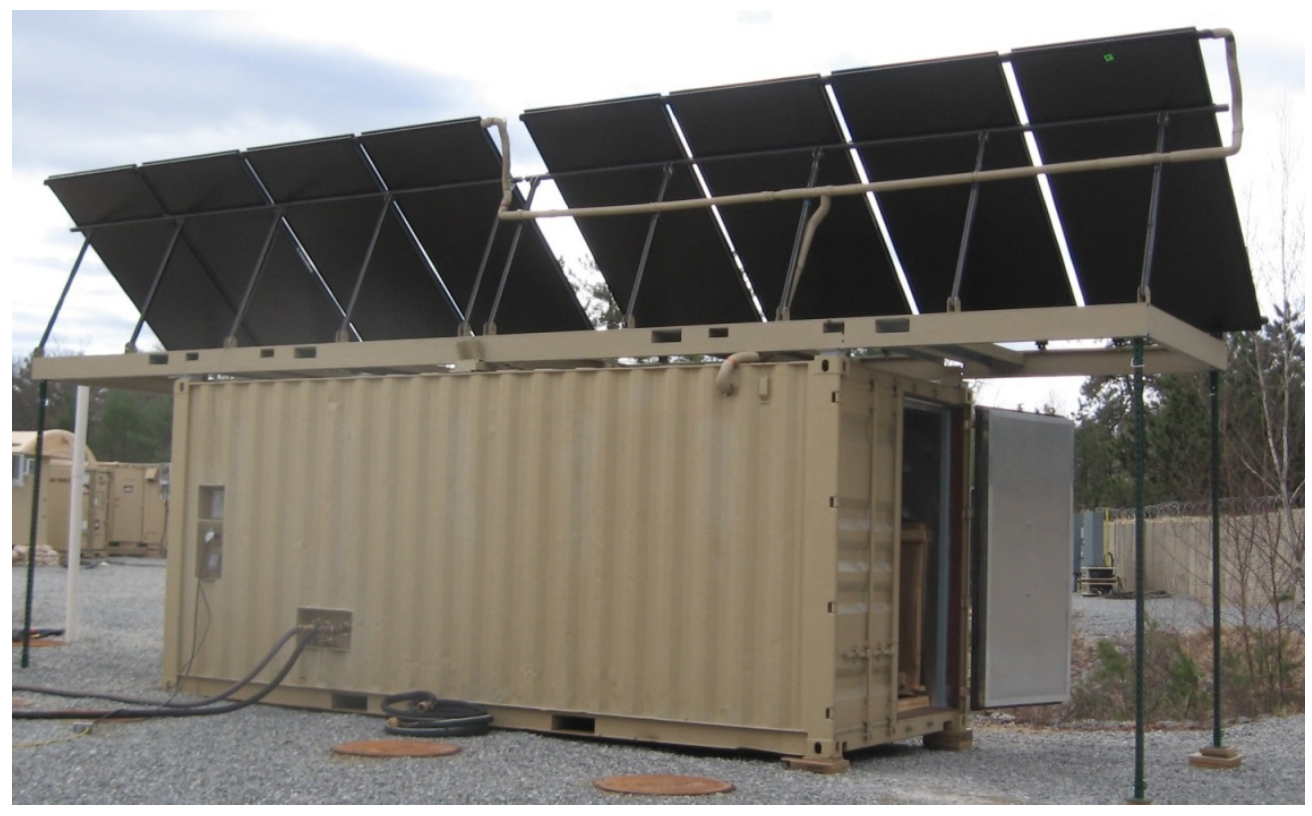

Figure A-21. SAGE SHW System Electrical and Water Connections

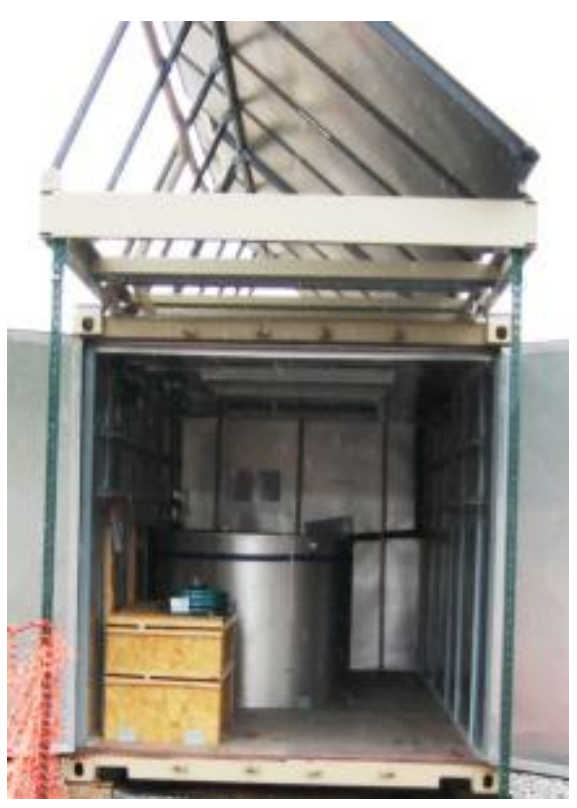

Figure A-22. Storage Tank, Controls, Lighting and Piping inside Container 


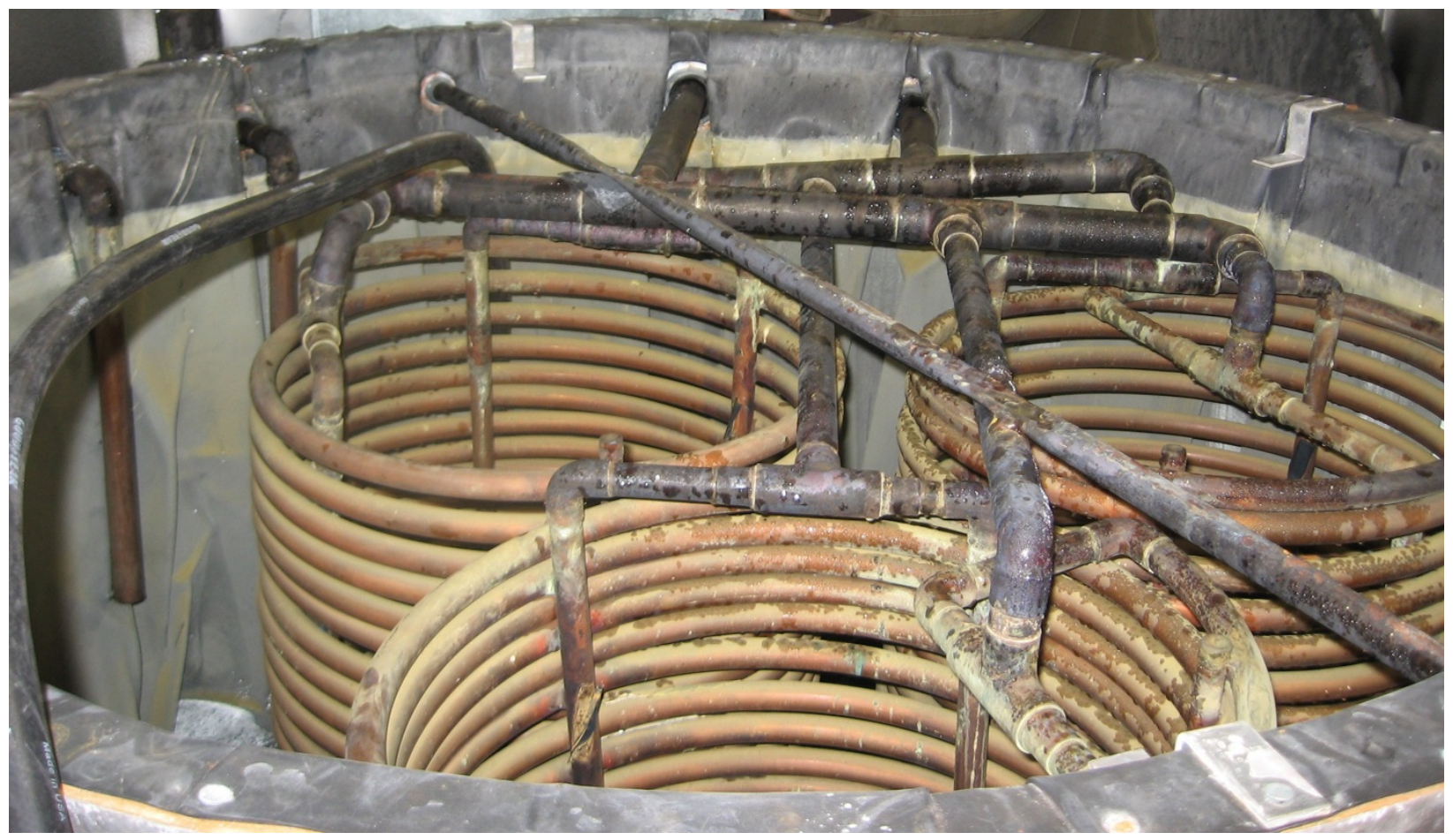

Figure A-23. Heat Exchanger Coils inside SHW System Storage Tank

\section{Energy Efficient Buildings}

\section{A.1.31 Description}

The SAGE buildings integrate several design features to improve energy efficiency and control:

- wall, floor, and ceiling materials with high insulation values

- low air-infiltration construction

- LED lighting

- fresh air ventilation with heat reclaim

- high efficiency heat/cool pump

- remotely controllable circuit breakers and thermostats.

SAGE also incorporated structural insulated panel (SIP) technology to demonstrate the ability to more quickly assemble and disassemble structures in the field.

Although SAGE focused on applying these features to billets, these features are useful to other building types, including administration, medical, classrooms and tactical operations centers. 
The key technology for reducing energy consumption is higher insulation and more efficient heat/cool pumps. Several of the recently published Army Standards documents for buildings already specify good insulation, including R-20 for walls.

\section{A.1.32 Specifications}

\section{A.1.32.1 Building Envelope}

- Provide the maximum amount of insulation that can be accommodated by the structure's envelope (walls, floors, ceilings). Consider, for example, that the B-Hut's standard design is $2 \times 4$ studs for walls and floors and $2 \times 6$ joists for ceilings/roofs, therefore, the specification is to add batt or foam board insulation between studs and joists. Consider also installing a layer of insulative sheathing to the outside face of the studs before installing the exterior weather sheathing. Also consider adding an interior sheathing to protect the insulation from removal and damage.

- Reduce air infiltration of the initial construction work to reduce air gaps in the building envelope. Consider using insulated doors and frames. Consider using higher efficiency windows that incorporate metal frames with thermal breaks or polyvinyl chloride (PVC) frames, and glazing that is double paned with gas fill and thermal film.

- Consider using SIP building systems to improve insulation and reduce infiltration, and to simplify the relocation of the building to other sites.

\section{A.1.32.2 Heat/Cool Pumps}

- Provide high efficiency heat pumps that both heat and cool air, and that have higher Seasonal Energy Efficiency Ratios (SEERs) than the average COTS heat pumps. As of the date of the SAGE report, the minimum SEER in the United States is 13. Consider the guidelines at http://energy.gov/energysaver/articles/central-air-conditioning provided by the U.S. Department of Energy.

- Consider providing unitary heat/cool pumps, commonly referred to as "through the wall AC," instead of "split systems." Split systems separate the heat/cool pump into an exterior unit for exchanging heat/cool with the outdoor air, and an interior unit for exchanging heat/cool with the inside air; these paired units are interconnected by a refrigerant line that requires specially trained and certified technicians to charge the refrigerant lines during the first installation and then for any subsequent refrigerant-related repairs, maintenance, replacements, and relocations. In addition, many unitary units have $120 \mathrm{~V}$ or $220 \mathrm{~V}$ power cords that an untrained person can plug and unplug into outlets, in contrast to higher voltage units that require an electrician to connect and disconnect the wiring. Because of these unitary unit features, a failed or poorly performing unitary unit can be simply replaced with another unitary unit without need for refrigerant technicians or electricians. 


\section{A.1.32.3 Controllable Equipment}

Thermostats

- Provide thermostats with GUIs that are programmable to set schedules for temperatures for each day of the week. Include in the GUI a one-step method to switch the temperature setting between occupied and unoccupied periods. Consider using occupancy sensors that are either separate units or integrated into the thermostat enclosures to also override the daily schedule for occupancy hours. Consider providing a lockable enclosure or other means to control access to the programming controls.

- For remotely controllable thermostats, provide thermostats with network interface and open protocols of Modbus or BACnet. Control the temperature, the fan, and the compressor independently.

\section{$\underline{\text { Circuit Breakers }}$}

- Provide remotely controllable circuit breakers to enable monitoring, load shedding, and other system controls at each building.

$\underline{\text { Remotely Controllable Equipment Monitoring and Control }}$

- For the equipment noted above and all other remotely controllable equipment, provide an Ethernet IEEE 802.11g or newer network interface.

- Include encryption per WPA2 that is configurable in the field.

- Refer to the SAGE Contingency Base Energy Management System specification for additional requirements and considerations, and for integrating the building with base operations.

\section{A.1.32.4 Ventilation}

- For buildings that are relatively air tight because of being recently built to DOD standards, or for existing buildings being retrofitted with insulation and infiltration control, provide a fresh-air ventilation system. Consider using a system with a heat exchanger to save energy. In cold seasons, the exchanger moves heat from the exhaust air into the incoming fresh air. In hot seasons, the exchanger moves heat from the incoming fresh air into the exhaust air.

\section{A.1.32.5 Lighting}

- Provide the currently most efficient lighting, which at the date of this report is LEDs.

- Consider providing light-level switching that allow users to set a low level of lighting for non-work-intensive periods. Light-level switches typically put one-half or two-thirds of a room's lights on one switch, and the remaining lights on a second switch.

- Consider providing a security blue light function for military security conditions.

- Provide motion-activated lights inside of vestibules for safe access. 


\section{A.1.32.6 Non-Energy Related SIP Specifications}

- Consider using SIPs for buildings that may need to be relocated several times by disassembly and truck transport.

- When using SIPs, provide SIPs with factory applied finishes and color for greater resistance to moisture damage, corrosion, and shipping wear.

- Provide a minimum insulation of R-18 for floors, R-22 for walls, and R-25 for roof.

- Consider common building code requirements for U.S. buildings, including using materials that are fire resistant Type II, noncombustible materials (International Building Code [IBC]).

- Design the building system to withstand rough handling during multiple assemblies and reassemblies over a 15-year lifespan.

- Package the system for shipping and storage in standard ISO $20 \mathrm{ft}$ containers.

- Design the components to be portable, modular, and quickly installed using a minimal set of tools.

\section{A.1.32.7 Monitoring and Control}

Refer to the SAGE Contingency Base Energy Management System specification for additional requirements and considerations, and for integrating the buildings with base operations.

\section{A.1.33 Scalability}

Stock or modular buildings are scalable to any sized base camp simply by increasing the quantities ordered to meet the needs of a larger camp. In addition, the building module can be increased to provide larger square footages and additional stories.

When existing TCMS buildings, such as B-Huts, are in place or planned for larger camps, then consider using the specifications for making energy efficiency improvements.

\section{A.1.34 References}

\section{A.1.34.1 Unified Facilities Criteria}

The UFC documents specify design criteria for many types of buildings and support infrastructure. This SAGE specification relates to most of the building types and the monitoringand-control related UFCs.

\section{A.1.34.2 Unified Facilities Guide Specifications}

- UFGS 0742 63, Fabricated Wall Panel Assemblies 


\section{A.1.34.3 Army Standards}

In addition to the UFCs and UFGS, the Army has begun a new series of design standards documents, each focused on a type of building. Incorporate the SAGE building specifications into these standards.

\section{A.1.35 Incorporate SAGE Technologies into Specifications}

- Revise the existing specifications for building types to include the requirements and options noted herein.

- The proprietary building products demonstrated during SAGE field tests may be useful to include as an "or equal" reference in the appropriate specifications, such as TCMS TOGS and ZZ-NSNs, for the purpose of defining the quality and the range of functions. Write the specification revisions or new sections to focus on the nonproprietary requirements and then include a statement like the following: "Provide a Mitsubishi MSZ GE 18NA Heat Pump or equal" for the key components not already specified in existing DOD specifications.

\section{A.1.36 Key Equipment Deployed in the SAGE Demonstration at BCIL}

\section{A.1.36.1 Building Envelope}

The SAGE BCIL billet buildings' floors, walls, and ceilings/roofs consisted of $2 \mathrm{ft}$ wide SIPs rated at R-25 manufactured by LEEP Inc., www.leepinc.com. Each building included a vestibule also consisting of LEEP panels. Although the panels had been rated by the manufacturer, no overall building R-value rating had been established by laboratory certification or field testing.

\section{A.1.36.2 Heat/Cool Pumps}

The SAGE BCIL heat/cool pump was the Mitsubishi MSZ GE 18NA Heat Pump, rated at SEER 19.2 with a cooling capacity range of 3,700-18,700 Btus, and a heating capacity range of 3,400-25,200 Btus. This unit includes humidity control based on temperature. More information can be found at the following link: www.mitsubishicomfort.com/media/359053/mseries\%20brochure\%203_11-r_pages.pdf.

\section{A.1.36.3 Controllable Equipment}

Thermostats

The SAGE heat/cool pump was controllable by the following methods: an included onboard thermostat, an included handheld wireless remote control, and a separately obtained wallmounted thermostat using hard-wired connections. For BCIL, SAGE used a wall-mounted programmable Mitsubishi PAR F27 hard-wired thermostat located in each energy efficient shelter. All Mitsubishi equipment was connected to the BCIL and CB-EMS network by a BACnet-compliant Mitsubishi BM adapter BAC-HD150. This adapter provided the Ethernet connection to the BCIL/CB-EMS system and interface to the M-net (Mitsubishi equipment 
network and 399IF-E Interface) for remote control and monitoring of the cooling/heating system. Additional information can be found at the following links:

http://www.mitsubishielectric.ca/en/hvac/PDF/city_multi/BAC-HD150_200910.pdf

www.mitsubishi-aircon.co.uk/default.asp?url=http\%3A//www.mitsubishi-

aircon.co.uk/mitsubishi_electric.asp\%3Fid\%3D175015

Circuit Breakers

Each SAGE billet building was equipped with a Schneider/Square-D Powerlink G3 system that features a microprocessor-based controller in an electrical panel to provide remotely controllable circuit breakers. The electrical panels were connected to the CB-EMS by Ethernet communications using Modbus TCP interfacing to a secured wireless local network. The panel meets NEC standards. The CB-EMS remotely controlled the breakers to demonstrate manual load shedding and automatic, prioritized load shedding.

The 100 amp panel is made up of the following circuit breakers as illustrated in Figure A-24:

- manually operated breakers:

- one 60 amp main/principal breaker

- two 20 amp breakers for spares. For one building, one of these two breakers powered the network communications switch that served all three buildings.

- manually and remotely controllable breakers:

- two 20 amp breakers, one for each string of wall outlets on the left and right sides of the building

- one 20 amp breaker for the lights

- one 20 amp breaker for the two ventilators

- two 20 amp heating, ventilating and air conditioning (HVAC) breakers, $220 \mathrm{~V}$, double pole breakers)

- four 20 amp breakers for spares 


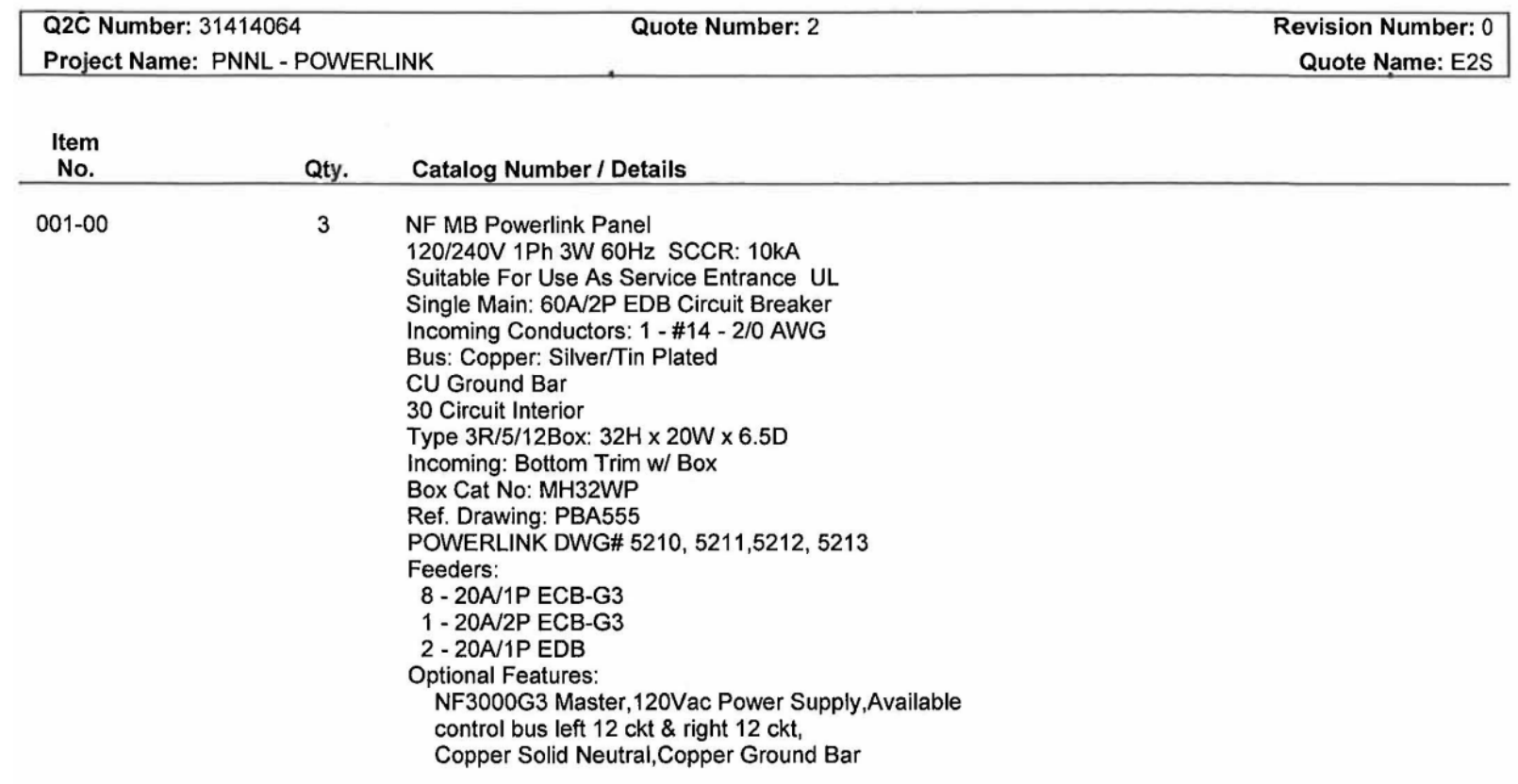

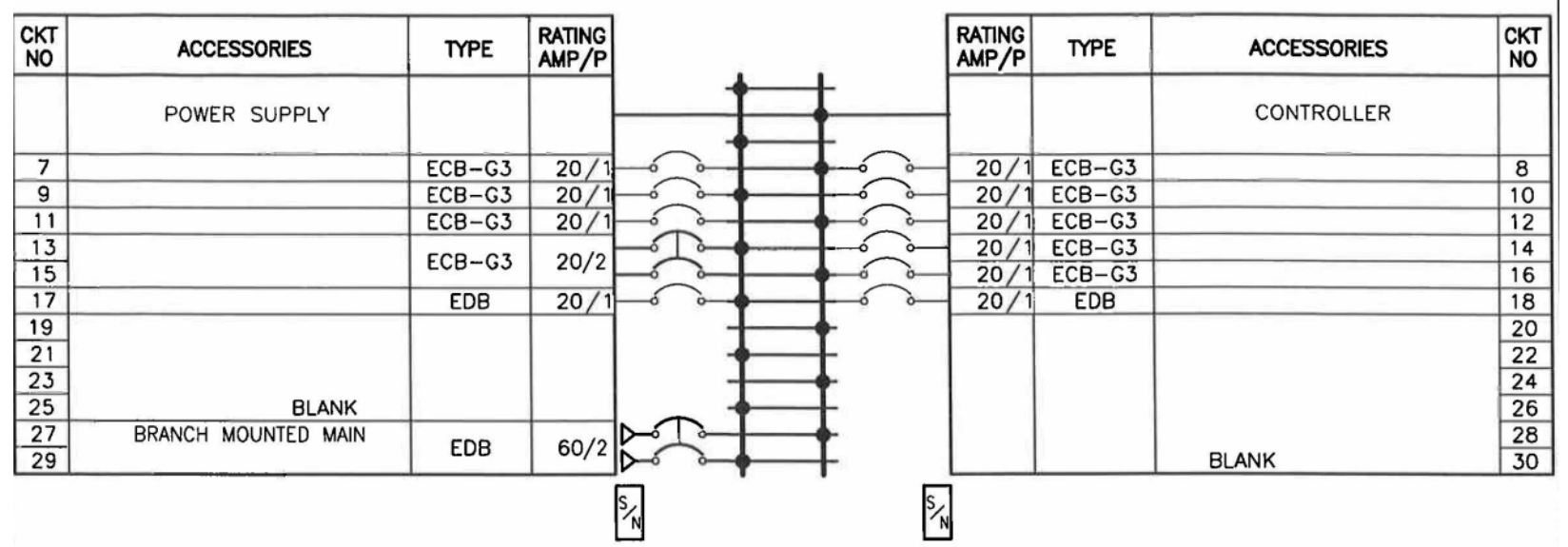

Figure A-24. 100 Amp Circuit Breaker Diagram from the Vendor's Design/Quotation

Additional information can be found at the following links:

- http://products.schneider-electric.us/products-services/products/lightingcontrol/powerlink/3000-level-system1/3000c-level-g3-controller/

- http://static.schneider-

electric.us/docs/Power\%20Management/Lighting\%20Control/Square\%20D\%20Powerlink\% 20Lighting\%20Control\%20Products/63249-401-205.pdf

Communications

The SAGE BCIL communications to the buildings' remotely controllable equipment was through a system developed and specified by the BCIL command. The key component was the RS900W switch by RuggedCom, http://www.ruggedcom.com/products/ruggedwireless/rs900w/, 
which is a hardened wireless Ethernet switch that functions as a stand-alone IEEE $802.11 \mathrm{~b} / \mathrm{g}$ access point for wireless clients providing wireless data rates of up to $54 \mathrm{Mbps}$. The RS900W includes strong encryption protocols using WPA with Temporal Key Integrity Protocol (TKIP) and WPA2/802.11i with Advanced Encryption Standard support. The CB-EMS/BCIL BACnet network monitored and controlled all of the circuit breakers and thermostats using the RuggedCom RS900W wireless Ethernet switch located on one shelter.

\section{A.1.36.4 Ventilation}

The SAGE BCIL building fresh air ventilation was provided by a pair of Broan HRV 90 ventilators with integral heat exchangers to minimize waste heat and to maintain improved humidity levels. The provider sized the ventilation for 22 occupants based on ASHRAE Standard 62.2-2010, Ventilation and Acceptable Indoor Air Quality for Low Rise Residential Buildings. The manufacturer's user guide noted the following features: each heat recovery ventilator has three settings: On, Low, and High, and recommended the following settings for various occupant loads: for four or fewer occupants, use one ventilator on Low setting; for 4-8 Occupants, use one ventilator on High setting; for 8-12 Occupants, use one ventilator on High and one ventilator on Low; for more than 12 occupants, use two ventilators on High setting.

\section{A.1.36.5 Lighting}

The SAGE BCIL building lighting consisted of 16 Techshot general purpose lights that provided 1,000 lumens per luminaire and used less than 9 watts per luminaire, for a total wholebuilding lighting load of 144 watts. The lights were on one circuit and switched to have three modes: Off, On, and Blackout. Each building had two light switches, one at the vestibule front door, and one at the back door.

\section{A.1.37 Photos of the Deployed SAGE Systems at BCIL}

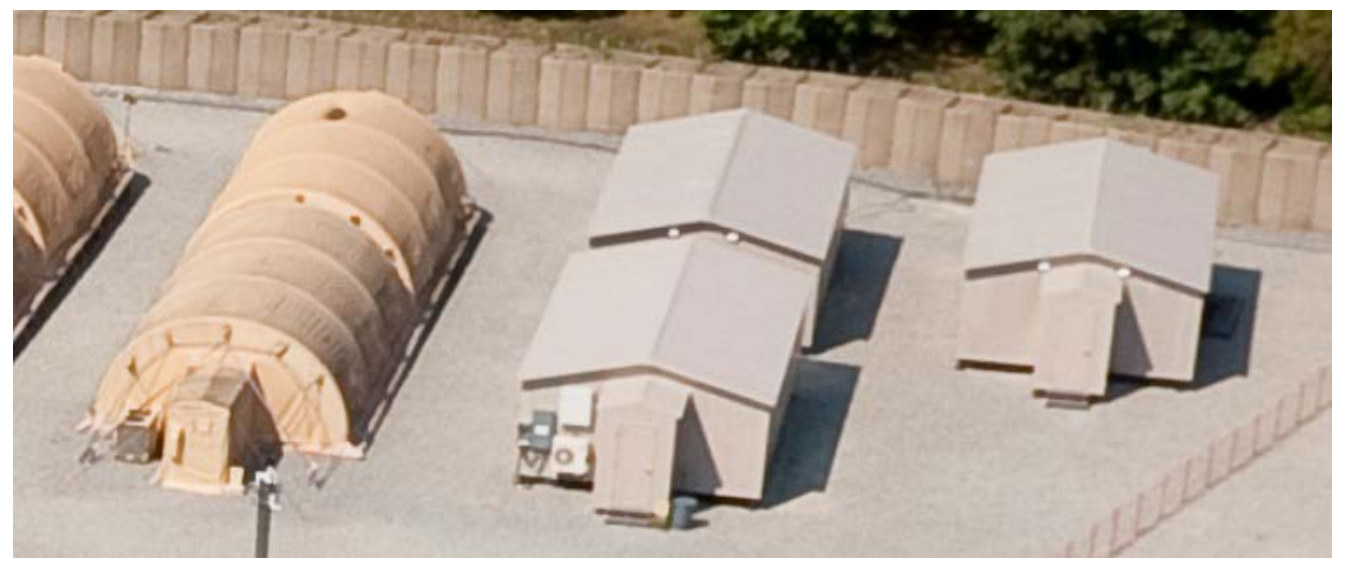

Figure A-25. Aerial Photo of the SAGE Billet Buildings at BCIL, adjacent to BCIL's Standard Air-Beam Tents Also Used for Billeting 


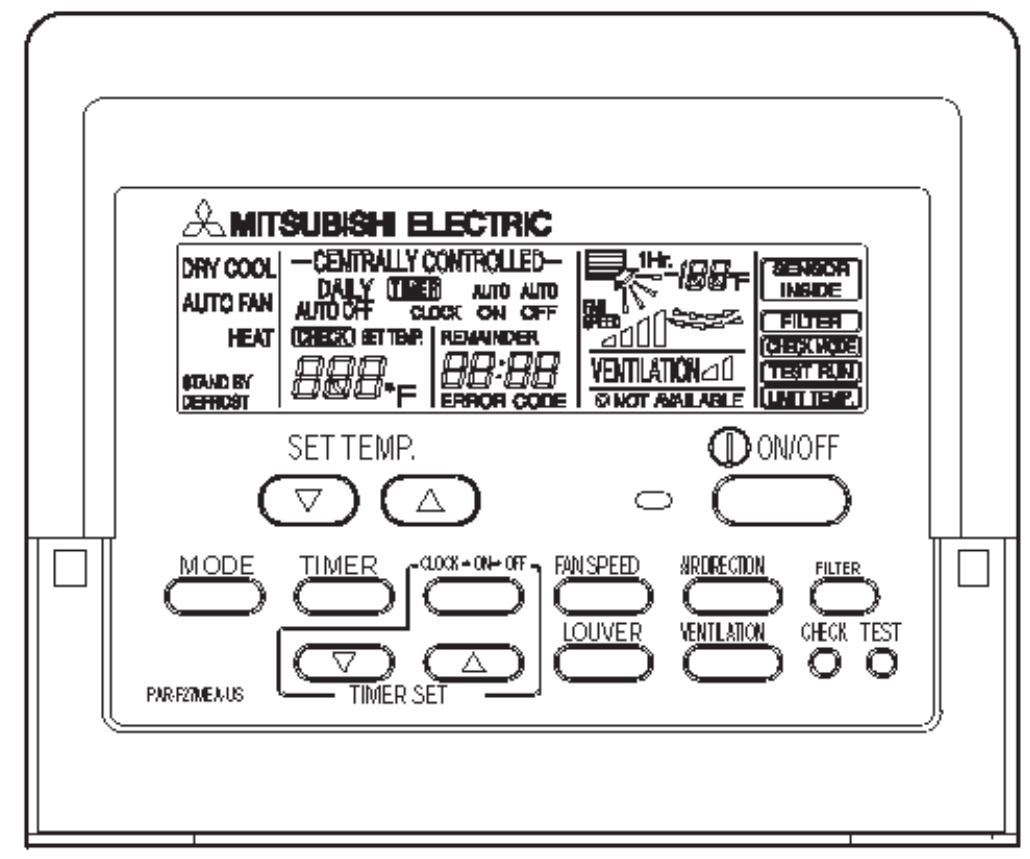

Figure A-26. Manufacturer's Diagram of the Mitsubishi Remotely Controllable Thermostat Used in Each SAGE Billet Building

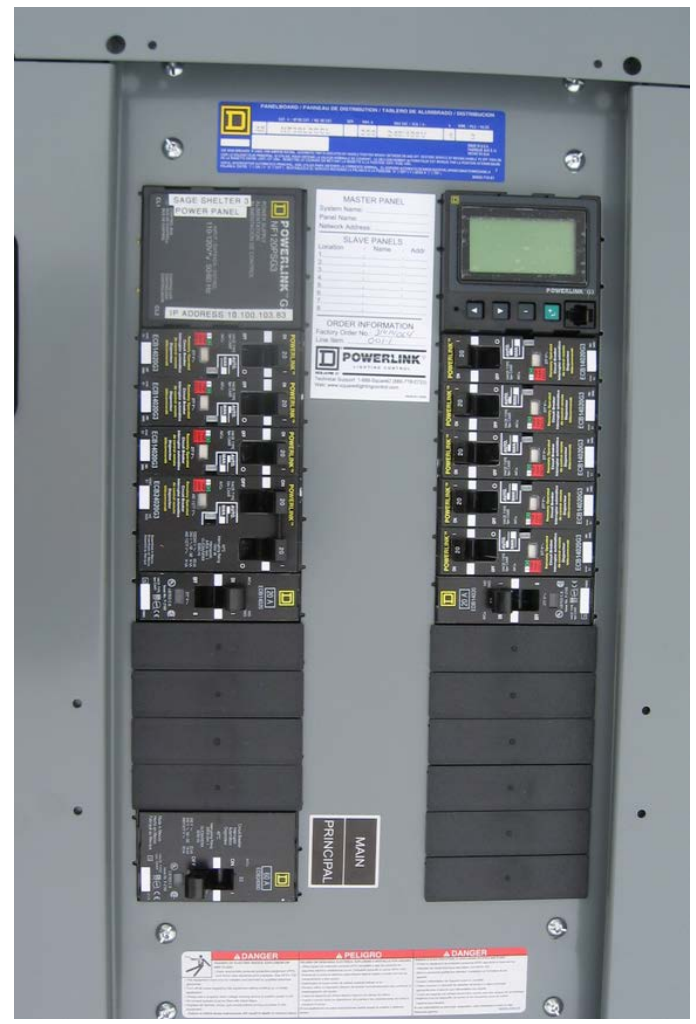

Figure A-27. The G3 Remotely Controllable Circuit Breakers Used for Each SAGE Billet Building. A small red-colored square on a breaker indicates that it is a remotely controllable breaker. The breakers without the red square are common, manualonly breakers. 


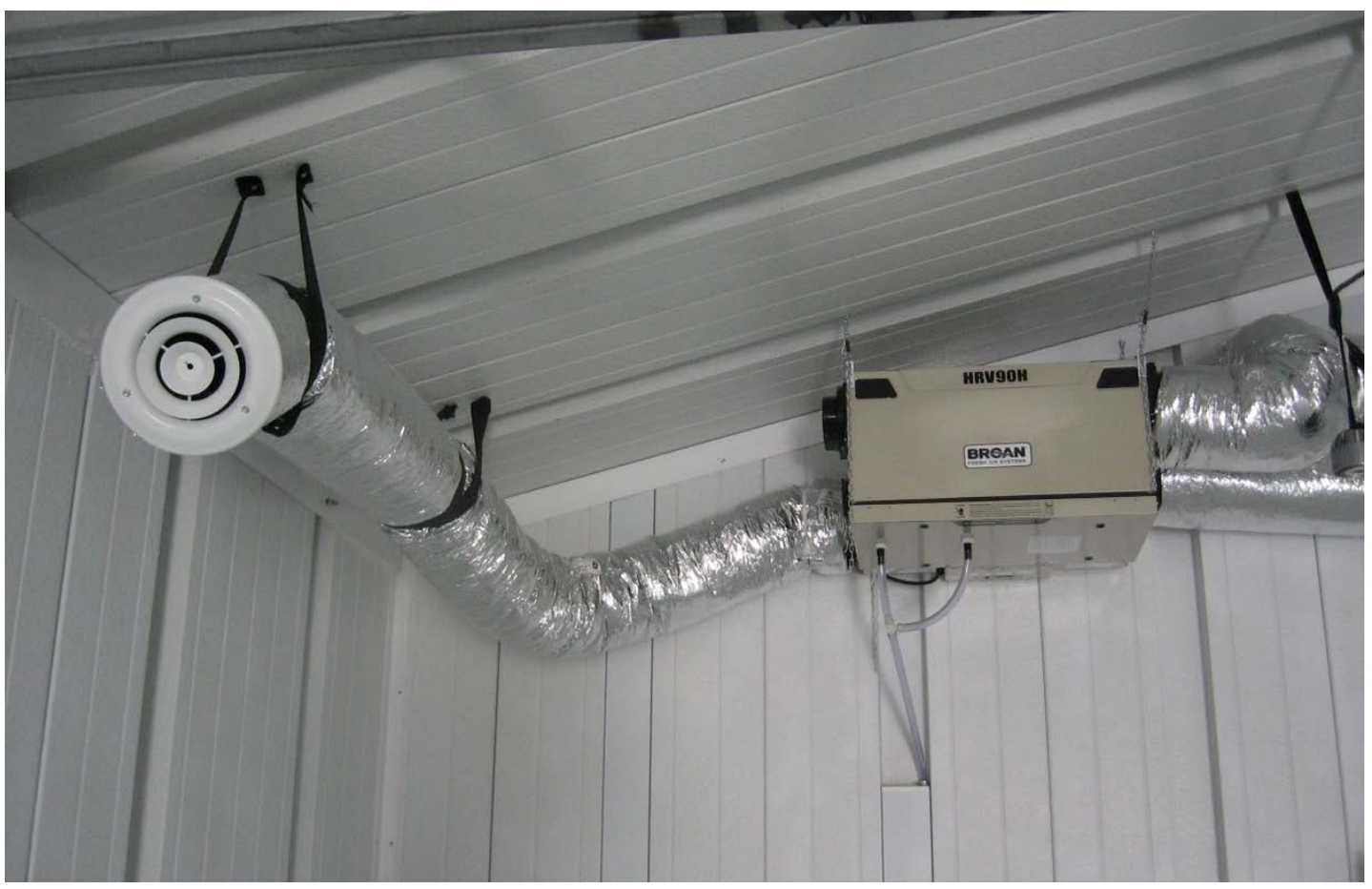

Figure A-28. One of Two Fresh Air Ventilation Units that Incorporated Heat Exchangers, as Installed in a SAGE Billet Building

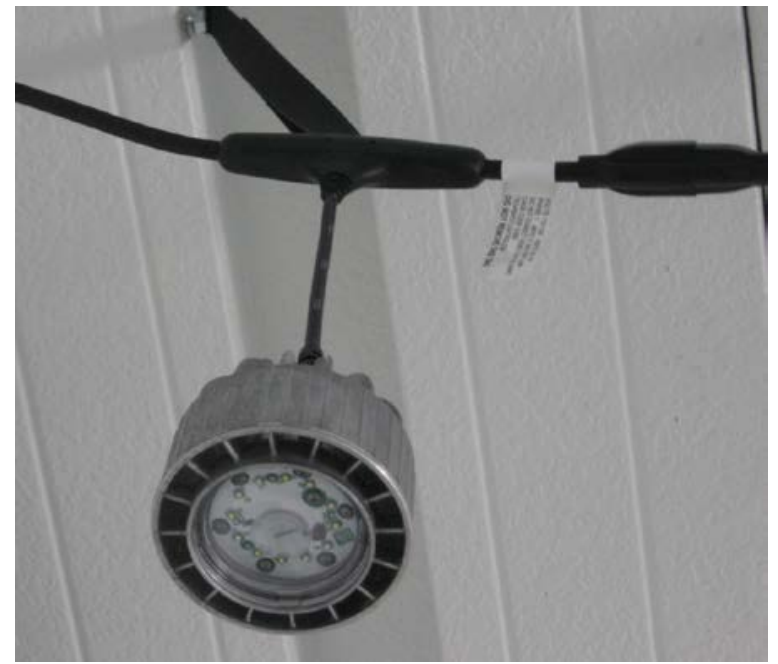

Figure A-29. One of the Billet Building LED Lights, Suspended from the Ceiling, and a DaisyChained Power Cable

\section{Contingency Base Energy Management System}

\section{A.1.38 Description}

The SAGE concept for a CB-EMS (using DSOM) addresses a comprehensive range of facility monitoring and control tools for many types of facilities. A robust CB-EMS supports the life cycle of a contingency base camp, from planning, design, construction, operations, 
maintenance, engineering, training, administration, annual reporting, and into the end-of-life disposition.

A robust CB-EMS includes the sets of functions that are referred to by several names, including Facility Monitoring and Control Systems (FMCS), Energy Monitoring and Control Systems (EMCS), Utility Monitoring and Control Systems (UMCS), and Supervisory Control and Data Acquisition (SCADA) systems, depending on the targeted types of facilities and equipment.

The energy related portion of the SAGE CB-EMS obtains, displays, stores, and analyzes energy performance data on base camp facilities and equipment. CB-EMS controls and diagnoses facilities and equipment to achieve improved performance and operations. Performance data quantifies utilities usage (e.g., fuel, potable water, wastewater), living space temperatures, electrical power consumption, electrical power generation, and any other relevant information that provides a sufficiently complete description of field operating base camp performance to enable optimization of base camp operation. A robust system can provide many additional functions, including fire protection and security functions and communications with work management controls, such as/including maintenance scheduling.

The key CB-EMS technologies are the following:

- open communication and control protocols for all facilities and equipment components, including meters, sensors, heating/cooling systems, and the power grid.

- open software to provide the CB-EMS functions noted herein.

- user-friendly monitoring and control screens (GUIs), with remote access and secure authorized user identification.

Special Reference

The UFC and UFGS have several existing specification sections directly related to specifying systems like a CB-EMS. In particular, UFGS 2510 10, "Utility Monitoring and Control System (UMCS) Front End Integration" substantially defines technical requirements for user interfaces and open protocols. Many of the SAGE CB-EMS requirements are in the existing UFGS specifications but are repeated herein to document a robust and advanced CB-EMS.

\section{A.1.39 Specifications}

\section{A.1.39.1 User Interface}

- Provide a mouse-operated, icon-based GUI that displays simplified depictions of the base camp facilities and equipment. Partition the GUI into distinct visualizations, that is, software windows or screens that provide different detail and perspectives of base camp facilities and equipment. Organize the GUI screens into hierarchies such that the amount of detail increases on narrower perspectives of base camp facilities and equipment as the GUI user 
descends the hierarchy. Label all objects to identify all field device instruments and all key facilities and equipment (e.g., building name and number).

- Utilize web-based browser HTML programming that is compatible with accepted operating systems and utilizes industry-standard file formats.

- Provide multi-user, secure access for local and remote users to simultaneously access the GUIs, data, reports, controls, and other tools noted herein.

- Provide GUIs to modify existing and to create new GUIs, visual elements, data indicators, control inputs, and other tools noted herein.

\section{A.1.39.2 Data Acquisition}

- Automatically and regularly obtain real-time data from the available base camp field instruments, including the following equipment or systems:

- power meters on all power generation sources, major facilities, and major facility subsystems, including circuit breakers, thermostats, lights, and HVAC systems and components. Include the data available from electrical meters that provide phase voltage, current, and power factor.

- fuel meters on generators and other fuel consuming equipment

- potable water flow meters and water temperature sensors, including system pressures and fill levels

- wastewater flow meters and fill levels

- on-site or nearby weather station data

- Directly sample the field device instrument data at a predefined period ranging from at least one second to 15 minutes, depending on specific needs. Provide a means to protect data integrity during disruptions of power and communications. Display the real-time data on the GUIs using various visual elements such as/including digital indicators, dials, fill icons, and graphs.

\section{A.1.39.3 Monitoring and Control}

- Provide GUIs to enable users to select facilities and components for monitoring and control, including generators, environmental control units, thermostats, and remote circuit breakers. Provide GUIs to enable users to select data points for monitoring, control, and diagnostics; provide immediate responsiveness to selection and deselection of points. Provide GUIs to perform manual overrides, start and stops, and load shedding. Provide GUIs to change set points, trigger limits, alarm limits, and other diagnostic settings.

- Provide GUIs to indicate the occurrence of off-normal conditions in real time, including GUI indicators of the conditions and alarms using flashing, color-coded icons where the color coding reflects the severity of the alarm. Provide GUIs to obtain additional information 
related to the alarm, including a description of the alarm and the associated field device instruments, facilities, and equipment. Provide GUIs to display a list of all active alarms and to clear, disable, or inhibit one or more alarms.

- Provide GUIs to configure the automatic sending of alarm notifications to user-entered cell phones and email addresses.

- Provide GUIs to define off-normal conditions as excursions of parameters (field device instrument, performance, and utility data) above or below user-defined limits. Include the ability to select and define more than one pair of limits. Include the ability to define at least three ranges for a parameter, and to associate each range with a different alarm severity.

- Provide GUIs to control remote circuit breakers, modify environmental control unit temperature and fan set points, and adjust other controllable equipment to support load shedding. Provide GUIs to select controlled equipment and set prioritization for load shedding, and to group the selected equipment into unlimited load-shedding tiers. Provide GUIs to define automatic shedding.

\section{A.1.39.4 Data Analysis and Reporting}

- Provide GUIs to select data for, and display summaries of, energy consumption, water consumption, energy profiles, and energy forecasting for hourly, daily, monthly, and annual periods. Provide GUIs to produce GUI screens, printouts, and data exports of the summaries.

- Display field instrument, performance, and utility data in standard units, including the following:

\begin{tabular}{ll}
\hline Generator power & $\mathrm{kW}$ \\
\hline Billet power consumption & $\mathrm{kW}$ \\
\hline Generator fuel flow & $\mathrm{gpm}$ \\
\hline Potable water flow & $\mathrm{gpm}$ \\
\hline Dining facility (DFAC) power consumption & $\mathrm{kW}$ \\
\hline Potable water temperature & ${ }^{\circ} \mathrm{F}$ \\
\hline Billet temperature & ${ }^{\circ} \mathrm{F}$ \\
\hline Outdoor temperature & ${ }^{\circ} \mathrm{F}$ \\
\hline Hot water heater fuel consumption & $\mathrm{gpm}$ \\
\hline Generator run state & on/off \\
\hline Generator run time & hours \\
\hline Waste water pump run state & on/off \\
\hline Electrical energy usage & $\mathrm{kWh}$ \\
\hline Potable water usage & $\mathrm{gal}$ \\
\hline Generator efficiency & $\%$ \\
\hline
\end{tabular}


- Provide GUIs to select field instruments for display of trend graphs versus user-selected time periods. Provide GUIs to graph multiple parameters on a single graph. Provide GUIs to store the customized graphs for later recall.

- Automatically and regularly calculate the selected totalized utility data, including energy consumption, from field device instrument data for display, reporting, and storage. Sample the data on an interval of 15 minutes or less.

- Automatically and regularly calculate selected performance data, including energy efficiencies, from field device instrument data for display and storage. Calculate this information on a data collection interval of one hour or less.

- Provide reports that provide all the data and analyses required by the end-user organization for reporting energy and water use and efficiencies to upper levels of command.

- Provide environmental monitoring to comply with, and document compliance with, regulations and policies.

\section{A.1.39.5 Long-Term Data Management}

- Provide GUIs to select field instruments and data collection intervals for long-term data storage. Provide default selection of instruments and intervals that can be unselected by users. Automatically compile the data into long-term storage. Compile the data in databases that are accessible by open-source database software. Provide storage for at least 90 days; consider providing storage for five-year intervals, up to the life of the facility being monitored.

- Provide data storage systems that use commercial technologies such as RAID (Redundant Array of Inexpensive Disks) to protect data against disk drive hardware failure. Provide backup, redundant, and/or uninterruptible power supplies for a minimum of three hours of power.

- Provide GUIs to generate the reports and analyses noted herein using the stored data.

\section{A.1.39.6 Diagnostics}

- Automatically and regularly perform and deliver diagnostics on base camp facilities and equipment for alerting operators of nonoptimal or abnormal conditions and of current and forecasted maintenance issues.

- Provide GUIs to obtain and to calculate data from field device instrument data and other derived data for display and to create and manage calculations and diagnostic processing. Provide GUIs to modify or add diagnostic algorithms and data processing scripts. Provide GUIs to display the results of the diagnostics, including problem details, the potential impacts, suggested likely causes and corrective actions. Provide GUIs to display estimated effects on energy use and costs. 
- Provide alarms of appropriate severity to indicate nonoptimal or abnormal conditions. Include in each generated alarm a description of the problem, a list of the potential impacts, a list of the likely causes, and the recommended corrective actions. Provide GUIs to select and prioritize alarm types to indicate the severity of the condition. Provide storage of alarm information for future retrieval, analysis, and trending.

- Provide whole-building electrical, gas, and water analyses to estimate baseline use for varying weather and occupancies, and predict expected values given a set of conditions, thus detecting deviations and notifying users of the appropriate action(s) to take to improve efficiency and reduce costs.

- Provide HVAC performance diagnostics to evaluate operation parameters and faults to make sure that the equipment is running correctly and to recommend improvement or corrective actions. Include diagnostics of damper functions, sensor failures, and operating set points. Include diagnostics of rooftop units.

- Consider providing diagnostics of HVAC outdoor air fraction and loss of air flow to determine cooling, heating, and economizer (using outdoor air to cool a building) conditions and failures. For cooling failures, diagnose improper temperature differences across the cooling coil as well as component failures including compressor, condenser fans, and additional devices that draw significant amperage. For heating failures, diagnose the improper temperature differences across the heating system as well as component failures including electric heat relays, gas valves, and other devices that draw significant amperage on the system.

- Consider monitoring all equipment for starts and stops. When monitoring equipment, totalize starts and stops to help determine that equipment is not short cycling or running excessively.

- Provide analyses of Occupied and Unoccupied settings to match actual occupancies. Include air handler fan system diagnostics to control fans and air temperatures to meet actual occupancies.

- Provide chiller and boiler plant diagnostics to optimize plant efficiency, predict maintenance actions, and avoid unplanned failures. Include diagnostics of pumping systems and of variable speed equipment, including motors and fans.

- Provide diagnostics for power generation systems. Provide GUIs to evaluate and control electrical load balancing across electrical phases and generators' efficiencies. Perform these diagnostic analyses on an interval no greater than 15 minutes. Include a function to forecast when to resupply base camp fuel supplies based on factors including past consumption patterns and occupancies, and past and forecasted weather.

\section{A.1.39.7 Exporting Data}

- Provide GUIs to export data, analyses, diagnostics, and other CB-EMS products noted herein in the following formats: Microsoft Excel $^{\circledR}$, comma separated values (CSV), and XML. 


\section{A.1.39.8 Communications}

- Provide a CB-EMS built on commercial and open hardware and software interface protocols of TCP/IP, Open Database Connectivity (ODBC), Object Linking and Embedding for Process Control (OPC), BACnet, LonWorks ${ }^{\circledR}$, Modbus (TCP, at a minimum) and others as applicable for the deployed type of meters and sensors. Interface to Microsoft SQL Server ${ }^{\circledR}$ and PostgreSQL ${ }^{\circledR}$. Consider interfacing with enterprise management systems, including Maximo $^{\circledR}$.

- Provide a reliable computer network for interfacing the CB-EMS with field device instruments, facilities, and miscellaneous equipment.

\section{A.1.39.9 Computational and Programming}

- Provide portability of the programing to a variety of platforms to accommodate a wide range of applications. Use a standard web application utilizing a standard web server and web browser. Provide for the use of applications that require a small, rugged, low-power platform where size, weight, and portability are important.

- Provide nonproprietary, open-source programming language, such as Python.

- Execute on Microsoft Windows ${ }^{\circledR} 7$ Service Pack 1 or newer, Microsoft Windows Server ${ }^{\circledR}$ 2008 R2 or newer, or an operating system approved by the using organization. Provide for expansion of functionality to include additional field device instruments, field device instrument data, performance data, utility data, GUI screens, diagnostics, notifications, reports, and data storage.

- Provide licensing that is flexible for the federal end-user to use on multiple and varying sizes of base camps and with large numbers of users.

\section{A.1.39.10 Cyber Security}

- Implement access control to limit access to approved operators using standard role-based security that can be implemented with Microsoft Active Directory ${ }^{\circledR}$ or local user authentication. Provide controls to limit the types of tools accessible to individual users and groupings of users, including individual user accounts.

- Obtain certification of the CB-EMS for deployment on local area networks of the end-user organization.

Installation and Commissioning

- Design the CB-EMS to function as originally designed and deployed when individual facility/equipment components are replaced with similar components from other manufacturers.

- Provide licensing, documentation, configuration information, configuration tools, programs, and drivers for the system to remain with the Government such that the Government or their 
agents are able to perform repair, replacement, upgrades, and expansions of the system without subsequent or future dependence on the system's original provider.

- Install and configure so that the Government or their agents are able to perform repair, replacement, and upgrades of individual hardware without further interaction with the system's original provider.

- Include user and operator manuals completely describing installation, configuration, upgrade, operation, and maintenance of the CB-EMS. Make design information nonproprietary to freely inform and facilitate installation, maintenance, and interfacing to third-party systems. Include all source code for screens, databases, scripts, and algorithms.

- Commission the installed CB-EMS, including using all CB-EMS features to show that each feature works correctly at least 10 times in a row. Include the following components and functions:

- operation of GUIs

- user authentication and setup

- data acquisition and display

- performance and utility consumption calculations

- setup and operation of diagnostics and notifications

- reporting

- data storage and retrieval

- Document the commissioning findings and provide the documentation in Adobe ${ }^{\circledR}$ PDF or Microsoft Word ${ }^{\circledR}$ formats.

- Provide documentation (licensing, manuals, code, etc.) on a compact disk (CD); provide 10 copies of the $\mathrm{CD}$. Consider providing the documentation also on a password-protected, nonpublic web server for a period of no less than five years; include the web address on each page of documentation.

\section{A.1.39.11 Training and Support}

- Provide on-site training that covers all aspects of installation, configuration, operation, and maintenance of the CB-EMS. Include tutorials with example scenarios in cross-platform formats, such as PDF, Word, jpg, and MP4.

- Provide training refreshment and trouble-call help by a 24/7 phone service for one year after CB-EMS acceptance by the end-user organization. Assume one hour of phone service for each deployed data and control point. 


\section{A.1.40 Scalability}

A robust CB-EMS type of system can be expanded to accommodate any size base camp. Design the core CB-EMS for the original, small base camp to accommodate significant increases in the quantity of facilities, equipment, meters, and sensors. Include in the small-base-camp CBEMS most of the features that would be provided for a large base camp. As a base camp grows, add GUIs and GUI content to display and process the added field device instrument data and control functions. If diagnostics were not part of the original, small-base-camp CB-EMS, then add diagnostics from existing, available libraries and/or create new diagnostics to evaluate the added equipment. Add data storage, communication devices, and support services.

\section{A.1.41 References}

\section{A.1.41.1 Unified Facilities Criteria}

- UFC 3-410-02, LonWorks Direct Digital Control for HVAC and Other Local Buildings Systems

- UFC 3-430-11, Boiler Control Systems

- UFC 3-470-01, LonWorks Utility Monitoring and Control System (UMCS).

\section{A.1.41.2 Unified Facilities Guide Specifications}

- UFGS 230923.13 20, BACnet Direct Digital Control Systems for HVAC

- UFGS 2309 23, LonWorks Direct Digital Control for HVAC and Other Building Control Systems

- UFGS 230933.00 40, Electric and Electronic Control System for HVAC

- UFGS 230953.00 20, Space Temperature Control Systems

- UFGS 2510 10, Utility Monitoring and Control System (UMCS) Front End and Integration

- UFGS 2831 13.00 40, Fire Detection and Alarm Control, GUI and Logic Systems

- UFGS 2309 23, LonWorks Direct Digital Control for HVAC and Other Building Control Systems.

\section{A.1.42 Incorporate SAGE Technologies into Specifications}

- Revise the existing specifications that address the various types and applications of CB-EMS to include the additional CB-EMS specifications noted herein.

- The proprietary CB-EMS demonstrated during SAGE field tests may be useful to include as an "or equal" reference in the appropriate specifications, such as TCMS TOGS and ZZ-NSNs, for the purpose of defining the quality and the range of functions. The SAGE CB-EMS at the Fort Devens BCIL was a limited adaptation of the Decision Support for 
Operations and Maintenance (DSOM) software. The SAGE CB-EMS was successfully demonstrated at BCIL in 2012, and the BCIL command will continue to use and adapt the SAGE CB-EMS for their long-term BCIL operations.

- Write the specification revisions, or a new specification section, to focus on the nonproprietary requirements noted herein and then include the statement "Provide the Decision Support for Operations and Maintenance (DSOM) software package or equal."

\section{A.1.43 Key Equipment Deployed in the SAGE Demonstration at BCIL}

The SAGE CB-EMS used for field testing at BCIL was the DSOM software package developed by the U.S. Department of Energy's Pacific Northwest National Laboratory for several federal organizations and sites. A key DSOM subcomponent was Inductive Automation's commercial Ignition software which provides the functionality of SCADA systems, building automation systems, and HMIs or GUIs. A second key DSOM component was the open-source Python programming language used for writing programs for specific sites, facilities, and equipment. The third key component was the effective library of previously built DSOM modules that were available for adaptation for use at BCIL. The DSOM suite provides a flexible plug-and-play system to communicate with almost all commercial data acquisition systems and facility control systems. DSOM is scalable and can be used to access and manage energy and facilities at equipment, building, base camp, regional or national levels.

At BCIL, the DSOM CB-EMS interfaced with BCIL's system of Shark ${ }^{\circledR}$ electricity power meters, Omega flow meters for water and fuel, National Instruments temperature sensors, a Davis weather station, National Instruments wired and wireless communications for individual instruments, RuggedCom multifunction switches for overall wireless local area networking, and LabView software for data acquisition and analysis.

The SAGE CB-EMS computer was a COTS Windows 7 PC that was configured at PNNL and deployed at the BCIL site. The CB-EMS PC accessed SAGE and BCIL instruments and facilities through BCIL's wireless network. The PC was accessed remotely via the commercial broadband service provided by BCIL.

The BCIL command elected to continue using the SAGE CB-EMS as the long-term front end to BCIL's variety of meters, sensors, facilities controls, and analyses. BCIL will develop additional GUIs and modules to add to the CB-EMS to best support BCIL's operations.

\section{A.1.43.1 Contacts}

PNNL designed and deployed the SAGE CB-EMS at BCIL. The PNNL contacts are Darrel Hatley, 509-375-2136, and James Goddard, 509-371-6748.

\section{A.1.44 Photos of the Deployed Equipment}

The following images show several of the GUIs deployed by SAGE for the CB-EMS BCIL. A common term for several of the GUIs is "dashboard" because the GUIs provide an overall 
view of a building's or a system's operations and conditions in a way much like a vehicle's dashboard does for speed, oil level, and warning indicators.

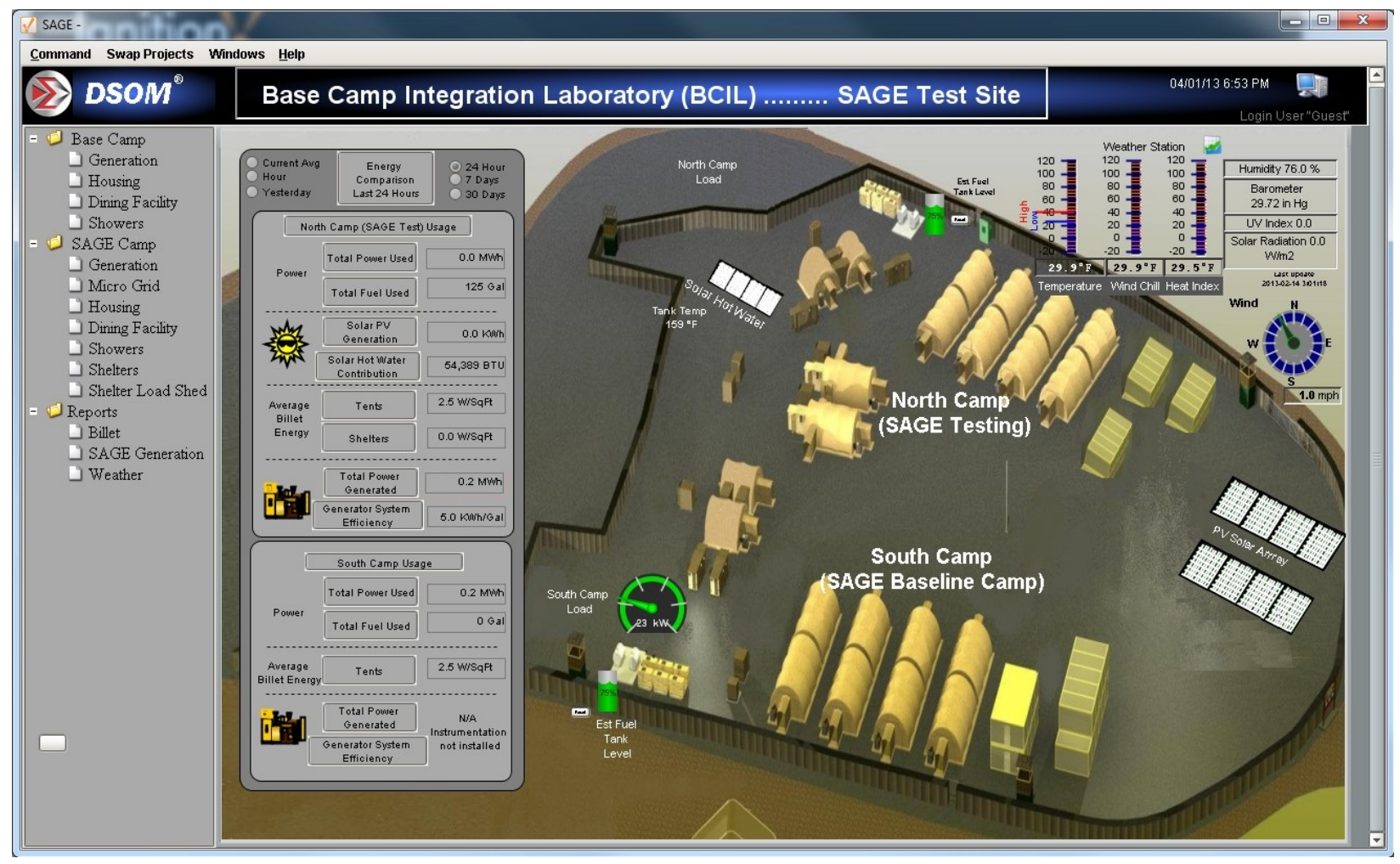

Figure A-30. The SAGE CB-EMS Home Screen or Dashboard GUI as Deployed for BCIL 


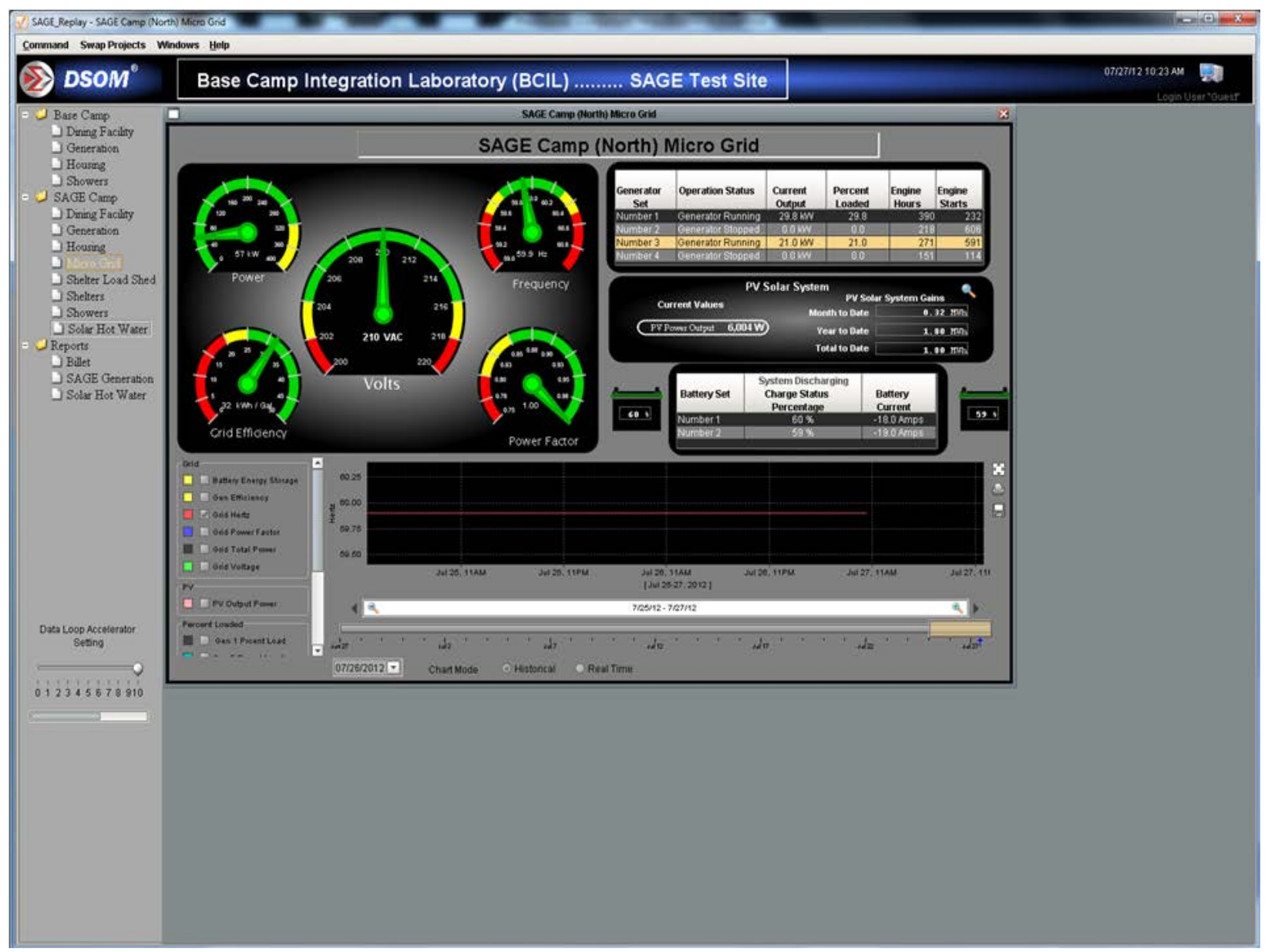

Figure A-31. The GUI Dashboard for the CB-EMS Microgrid 


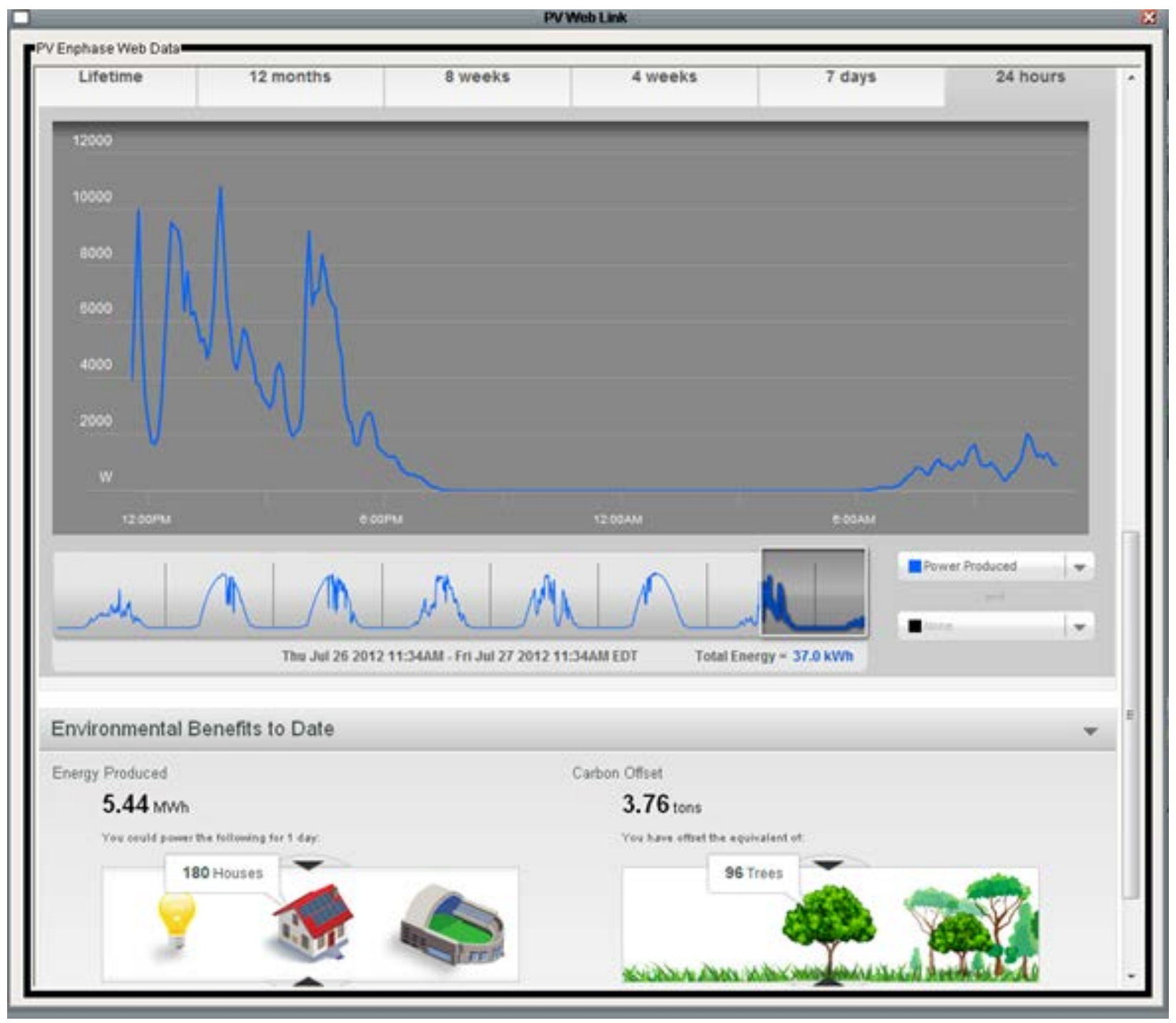

Figure A-32. The GUI for the CB-EMS PV which Presents the PV Microinverter Manufacturer's Live Website for the SAGE BCIL PV System 


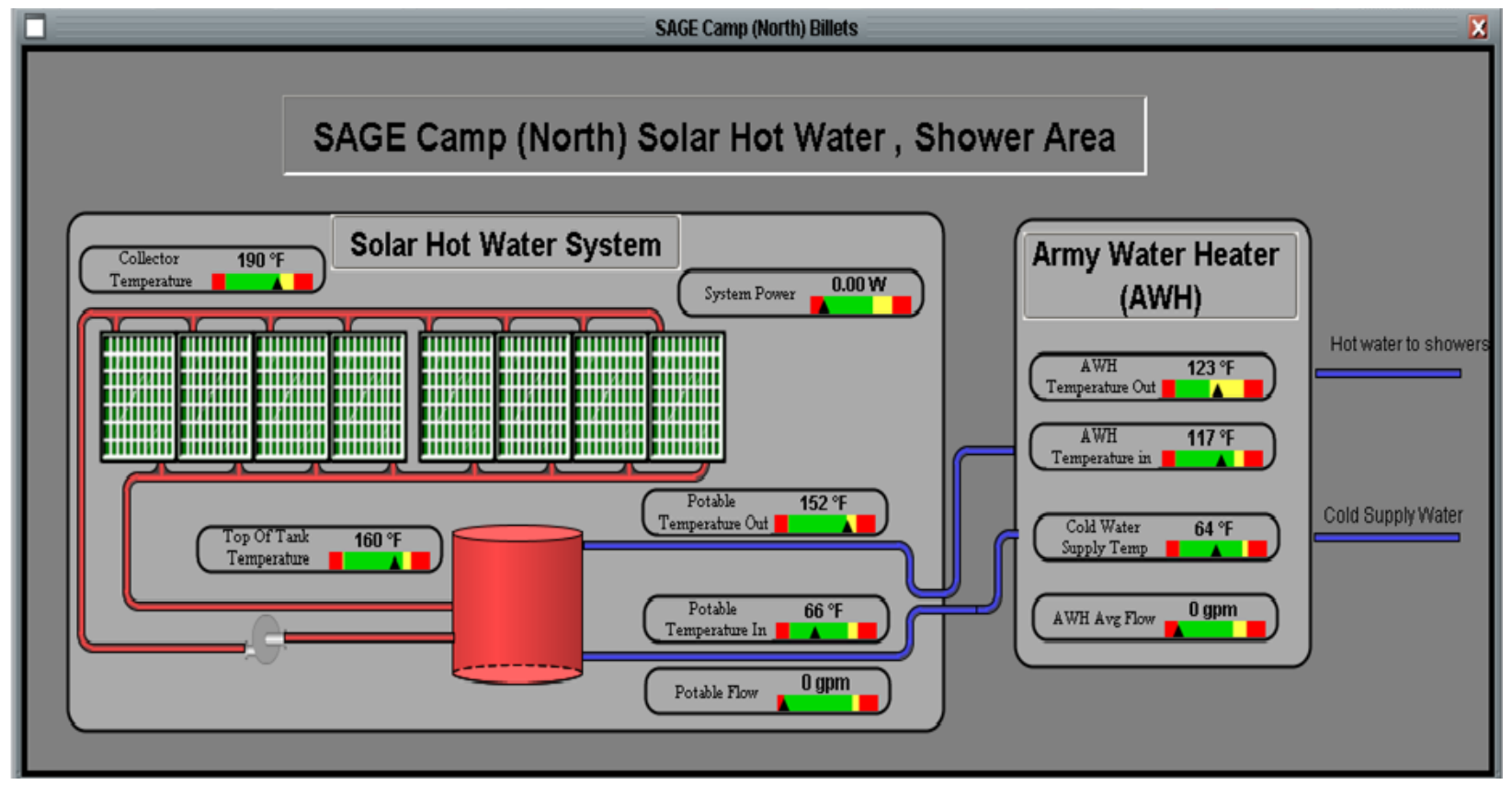

Figure A-33. The GUI for the CB-EMS Solar Hot Water System

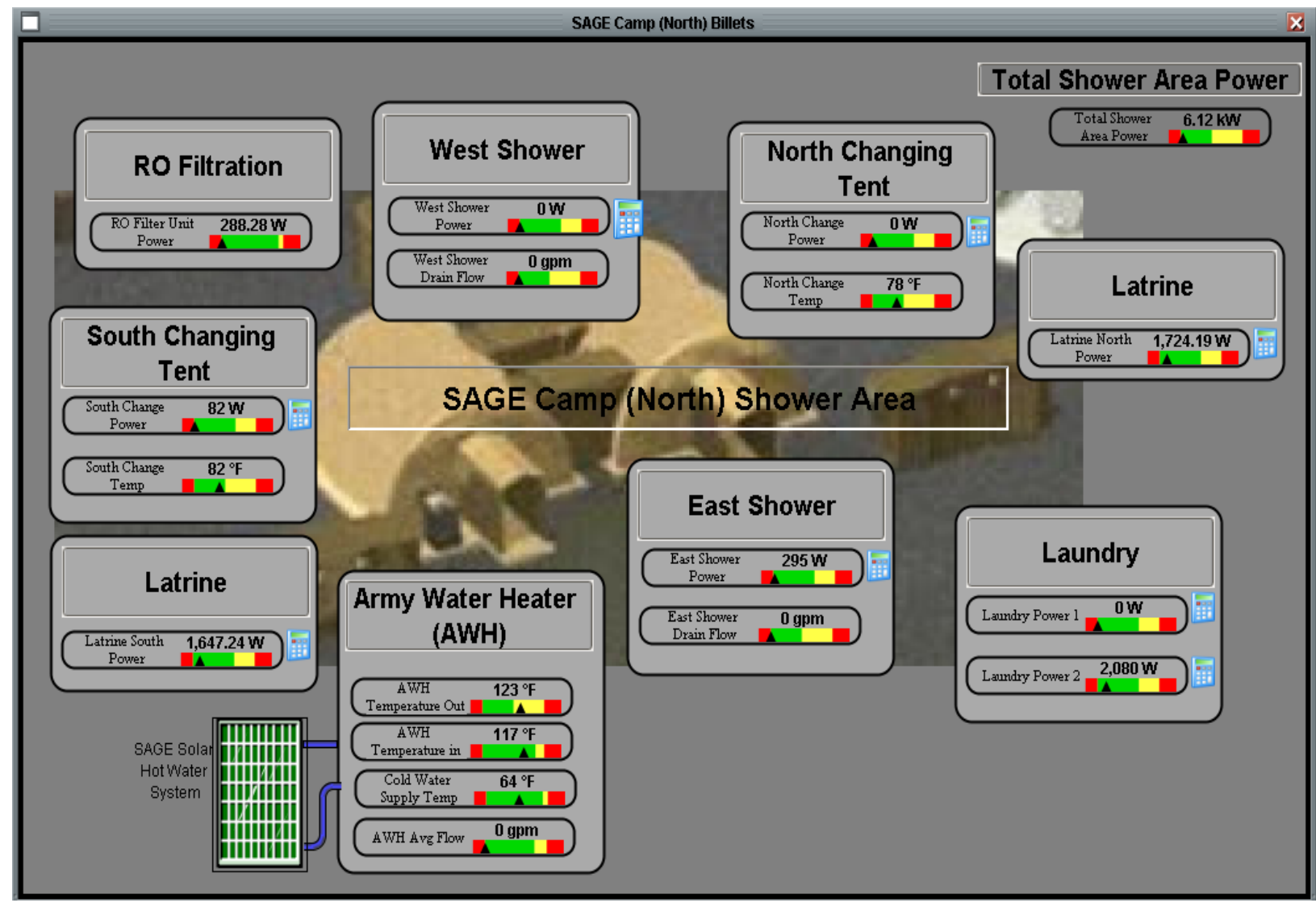

Figure A-34. The GUI for the CB-EMS Water System Temperatures, Including the Solar Hot Water System 


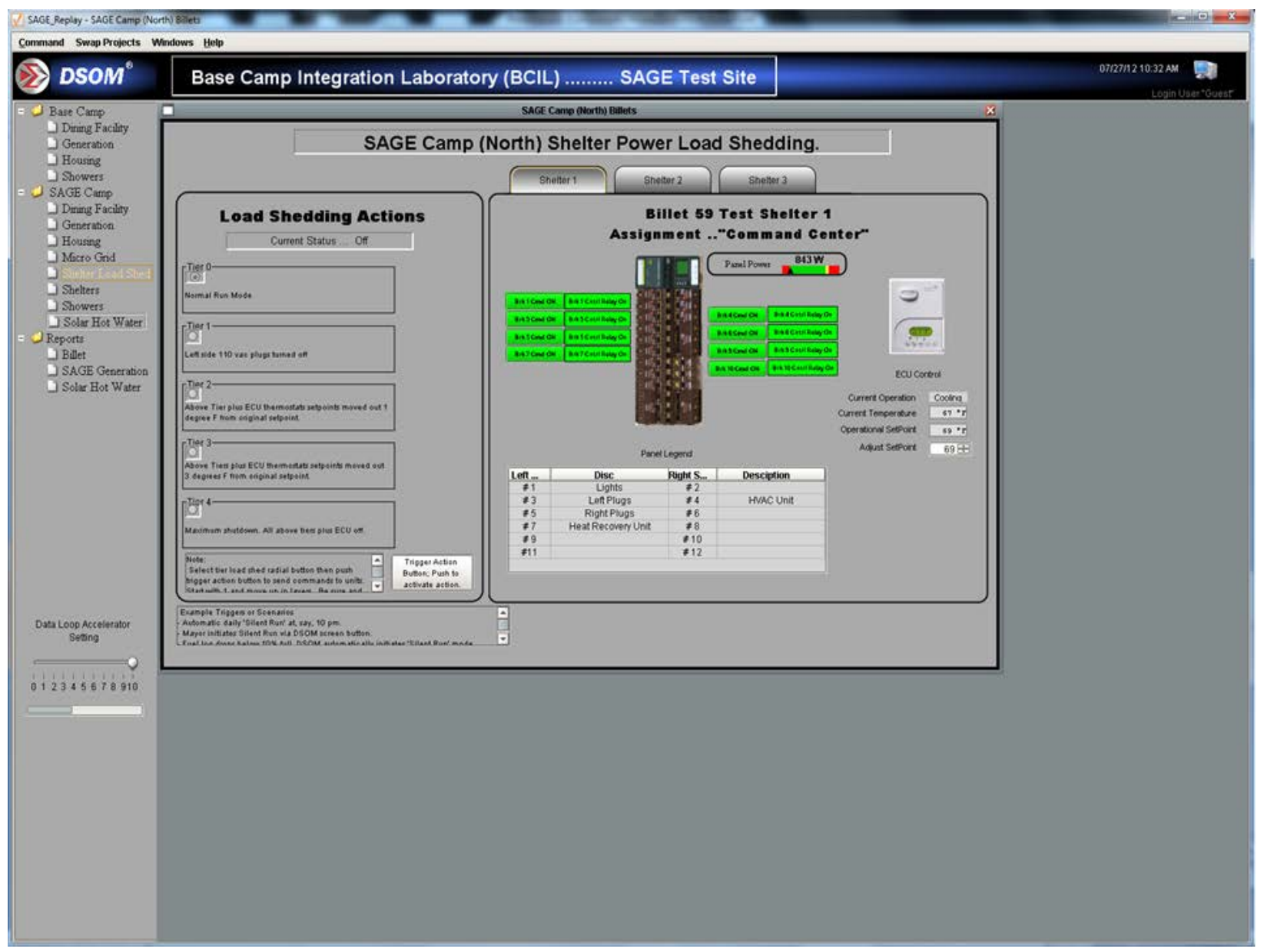

Figure A-35. GUI for the CB-EMS Remotely Controllable Equipment, which also shows the Automatic and Manual Load Shedding Controls Deployed for SAGE at BCIL 


\section{Appendix B \\ Structure Configuration of 150-, 600-, and 5,000-Soldier Camps}

This appendix describes the specific building or structure configuration for each of the three camp sizes considered for this report (150-, 600-, and 5000-Soldier camps). These configurations were used to establish energy and fuel figures for those camps worldwide.

\section{B.1 150-Soldier Camp}

The 150-Soldier camp, for the purposes of this report, was made up of the following structures:

- (8) billet troop housing tent, $20 \times 32 \mathrm{ft}, 640 \mathrm{ft}^{2}$

- (1) dining tent, $20 \times 32 \mathrm{ft}, 640 \mathrm{ft}^{2}$

- (1) freezer/refrigerator Tricon, $6.5 \times 8 \mathrm{ft}, 52 \mathrm{ft}^{2}$

- (1) kitchen Tricon, $6.5 \times 18.4 \mathrm{ft}, 120 \mathrm{ft}^{2}$

- (2) latrine Tricon, $6.5 \times 18.4 \mathrm{ft}, 120 \mathrm{ft}^{2}$

- (1) laundry Tricon, $6.5 \times 13 \mathrm{ft}, 85 \mathrm{ft}^{2}$

- (2) shower lobby tent, $20 \times 32 \mathrm{ft}, 640 \mathrm{ft}^{2}$

- (2) shower Tricon, $6.5 \times 18.4 \mathrm{ft}, 120 \mathrm{ft}^{2}$.

During field testing at Fort Devens, the camp utilized three modular, hard-walled, insulated shelters with the same footprint as the troop housing tents. For modeling purposes, all structures listed above were replaced with hard-walled, insulated structures for the "improved buildings" case. The 150-Soldier camp used the following options for power generation:

- six TQG-60 (60 kW) generators for spot generation

- three Caterpillar $100 \mathrm{~kW}$ generators plus a $250 \mathrm{kWh}$ battery bank linked together for a microgrid

- $13 \mathrm{~kW}$ photovoltaic arrays physical installation, $52 \mathrm{~kW}$ photovoltaic array for modeling.

\section{B.2 600-Soldier Camp}

The 600-Soldier camp, for the purposes of this report, was made up of the following structures:

- (1) AAFES retail store tent, $20 \times 32 \mathrm{ft}, 640 \mathrm{ft}^{2}$

- (2) admin tent, $20 \times 32 \mathrm{ft}, 640 \mathrm{ft}^{2}$

- (4) admin briefing tents, $20 \times 32 \mathrm{ft}, 640 \mathrm{ft}^{2}$ 
- (1) administrative tent, $20 \times 32 \mathrm{ft}, 640 \mathrm{ft}^{2}$

- (32) billet troop housing tents, $20 \times 32 \mathrm{ft}, 640 \mathrm{ft}^{2}$

- (1) chaplain tent, $20 \times 32 \mathrm{ft}, 640 \mathrm{ft}^{2}$

- (4) dining tents, $20 \times 32 \mathrm{ft}, 640 \mathrm{ft}^{2}$

- (4) freezer/refrigerator Tricons, $6.5 \times 8 \mathrm{ft}, 52 \mathrm{ft}^{2}$

- (2) guarding/badging tents, $20 \times 32 \mathrm{ft}, 640 \mathrm{ft}^{2}$

- (2) kitchen containers, $8 \times 20 \mathrm{ft}$ double expandable, $374 \mathrm{ft}^{2}$

- (8) latrine Tricons, $6.5 \times 18.4 \mathrm{ft}, 120 \mathrm{ft}^{2}$

- (4) laundry Tricons, $6.5 \times 13 \mathrm{ft}, 85 \mathrm{ft}^{2}$

- (3) medical tents, $20 \times 32 \mathrm{ft}$, $640 \mathrm{ft}^{2}$

- (1) morale, welfare and recreation (MWR) game room tent, $20 \times 32 \mathrm{ft}, 640 \mathrm{ft}^{2}$

- (1) MWR gym tent, $20 \times 32 \mathrm{ft}, 640 \mathrm{ft}^{2}$

- (1) MWR theater tent, $20 \times 32 \mathrm{ft}, 640 \mathrm{ft}^{2}$

- (4) security tents, $20 \times 32 \mathrm{ft}, 640 \mathrm{ft}^{2}$

- (8) shower lobby tents, $20 \times 32 \mathrm{ft}, 640 \mathrm{ft}^{2}$

- (8) shower Tricons, $6.5 \times 18.4 \mathrm{ft}, 120 \mathrm{ft}^{2}$

- (4) supply/exchange warehouse tents, $20 \times 32 \mathrm{ft}, 640 \mathrm{ft}^{2}$

- (8) vehicle maintenance tents, $20 \times 32 \mathrm{ft}, 640 \mathrm{ft}^{2}$.

For modeling purposes, all structures listed above were replaced with hard-walled, insulated structures for the "improved buildings" case.

The 600-Soldier camp used the following options for power generation:

- twenty-four TQG-60 (60 kW) generators for spot generation

- four Caterpillar $300 \mathrm{~kW}$ generators linked together for a microgrid

- $208 \mathrm{~kW}$ photovoltaic array.

\section{B. 3 5,000-Soldier Camp}

The 5,000-Soldier camp, for the purposes of this report, was made up of the following structures:

- (1) 2-XL admin bldg., $50 \times 120 \mathrm{ft}, 6,000 \mathrm{ft}^{2}$

- (1) AAFES storage bldg., $50 \times 120 \mathrm{ft}, 6,000 \mathrm{ft}^{2}$ 
- (57) admin bldg., $20 \times 60 \mathrm{ft}, 1,200 \mathrm{ft}^{2}$

- (84) barracks, $50 \times 120 \mathrm{ft}, 6,000 \mathrm{ft}^{2}$

- (4) barracks (command and staff housing), $50 \times 120 \mathrm{ft}, 6,000 \mathrm{ft}^{2}$

- (7) battalion HQ bldg., $50 \times 90 \mathrm{ft}, 4,500 \mathrm{ft}^{2}$

- (1) BDOC bldg., $50 \times 90 \mathrm{ft}, 4,500 \mathrm{ft}^{2}$

- (1) brigade HQ bldg., $50 \times 120 \mathrm{ft}, 6,000 \mathrm{ft}^{2}$

- (2) chapel, $50 \times 120 \mathrm{ft}, 6,000 \mathrm{ft}^{2}$

- (42) CO HQ bldg., $40 \times 50 \mathrm{ft}, 2,000 \mathrm{ft}^{2}$

- (1) dental facility, $50 \times 120 \mathrm{ft}, 6,000 \mathrm{ft}^{2}$

- (6) dining-kitchen, $50 \times 120 \mathrm{ft}, 6,000 \mathrm{ft}^{2}$

- (1) DPW bldg., $40 \times 50 \mathrm{ft}, 2,000 \mathrm{ft}^{2}$

- (1) entry control point - commercial, visitor center, $32 \times 50 \mathrm{ft}, 1,600 \mathrm{ft}^{2}$

- (1) entry control point - main, visitor center, $32 \times 52 \mathrm{ft}, 1,664 \mathrm{ft}^{2}$

- (1) finance bldg., $40 \times 50 \mathrm{ft}, 2,000 \mathrm{ft}^{2}$

- (2) general admin bldg., $50 \times 90 \mathrm{ft}, 4,500 \mathrm{ft}^{2}$

- (1) gymnasium, $150 \times 300 \mathrm{ft}, 45,000 \mathrm{ft}^{2}$

- (2) in-processing - civilian, $50 \times 90 \mathrm{ft}, 4,500 \mathrm{ft}^{2}$

- (1) intel bldg., $50 \times 60 \mathrm{ft}, 3,000 \mathrm{ft}^{2}$

- (1) JOC (TOC) bldg., $50 \times 90 \mathrm{ft}, 4,500 \mathrm{ft}^{2}$

- (1) kennel MP bldg., $50 \times 120 \mathrm{ft}, 6,000 \mathrm{ft}^{2}$

- (3) large admin bldg., $50 \times 90 \mathrm{ft}, 4,500 \mathrm{ft}^{2}$

- (14) latrine container, $8 \times 20 \mathrm{ft}, 160 \mathrm{ft}^{2}$

- (1) Mayor's cell bldg., $40 \times 50 \mathrm{ft}, 2,000 \mathrm{ft}^{2}$

- (7) medium admin bldg., $40 \times 50 \mathrm{ft}, 2,000 \mathrm{ft}^{2}$

- (1) mortuary affairs bldg., $50 \times 120 \mathrm{ft}, 6,000 \mathrm{ft}^{2}$

- (1) mortuary personal effects depot, $50 \times 200 \mathrm{ft}, 10,000 \mathrm{ft}^{2}$

- (1) mortuary refrigerated trailer, $30 \times 150 \mathrm{ft}, 4,500 \mathrm{ft}^{2}$

- (5) MWR bldg., $50 \times 90 \mathrm{ft}, 4,500 \mathrm{ft}^{2}$

- (1) provost MP bldg., $50 \times 120 \mathrm{ft}, 6,000 \mathrm{ft}^{2}$ 
- (1) public affairs bldg., $50 \times 60 \mathrm{ft}, 3,000 \mathrm{ft}^{2}$

- (1) range - M16 - bldg., $16 \times 32 \mathrm{ft}, 512 \mathrm{ft}^{2}$

- (1) range - M16 - latrine, $10 \times 16 \mathrm{ft}, 160 \mathrm{ft}^{2}$

- (1) range - M16 - tower, $11 \times 11 \mathrm{ft}, 121 \mathrm{ft}^{2}$

- (88) shower/latrines, $20 \times 40 \mathrm{ft}, 800 \mathrm{ft}^{2}$

- (1) supply bldg., $40 \times 50 \mathrm{ft}, 2,000 \mathrm{ft}^{2}$

- (7) T.O.C. bldg., $40 \times 50 \mathrm{ft}, 2,000 \mathrm{ft}^{2}$

- (1) TMC facility, $50 \times 120 \mathrm{ft}, 6,000 \mathrm{ft}^{2}$

- (14) vehicle maintenance bldg., $82 \times 212 \mathrm{ft}, 17,384 \mathrm{ft}^{2}$

- (3) warehouse bldg., $100 \times 500 \mathrm{ft}, 50,000 \mathrm{ft}^{2}$.

For modeling purposes, all structures listed above were replaced with hard-walled, insulated structures for the "improved buildings" case.

The 5,000-Soldier camp used the following options for power generation:

- nine 3,000 kW generators for spot generation, each serving nine equally sized sectors in the camp

- four 3,000 kW generators of the same type, linked together for a microgrid

- $1,716 \mathrm{~kW}$ photovoltaic array. 


\section{Appendix C Detailed Field Testing Methodology}

\section{Phase 1 Tests}

Phase 1 testing of the Smart and Green Energy (SAGE) microgrid was performed at the Caterpillar Technical Center in Mossville, Illinois, from February 14-February 16, 2012, with the goal of verifying and evaluating the design of the microgrid.

The scope of the testing included three Caterpillar XQ100 generators and associated EMCP 4.4 controllers; a battery system, including charger and bidirectional inverter; a photovoltaic (PV) system and microinverters; and the interfacing of these elements. The load was simulated by a programmable load bank with both resistive and inductive load elements.

The 24-hour load profiles defined in Table C-1 and depicted in Figure C-1 were used for 24-hour fuel consumption testing of the microgrid system.

Table C-1. Load Profiles

\begin{tabular}{|c|c|c|c|c|c|c|c|c|c|c|c|c|}
\hline \multicolumn{13}{|c|}{ Test Segment A } \\
\hline Hour & 1 & 2 & 3 & 4 & 5 & 6 & 7 & 8 & 9 & 10 & 11 & 12 \\
\hline Overall (kW) & 88.2 & 88.9 & 83.5 & 81.8 & 93.7 & 131.4 & 103.2 & 121.0 & 132.8 & 188.1 & 234.5 & 224.0 \\
\hline Kitchen $(\mathrm{kW})$ & 22.4 & 22.4 & 22.4 & 22.4 & 34.3 & 36.6 & 39.5 & 39.5 & 44.8 & 58.2 & 74.6 & 59.7 \\
\hline Constant $(\mathrm{kW})$ & 100 & 100 & 100 & 100 & 100 & 100 & 100 & 100 & 100 & 100 & 100 & 100 \\
\hline \multicolumn{13}{|c|}{ Test Segment B } \\
\hline Hour & 13 & 14 & 15 & 16 & 17 & 18 & 19 & 20 & 21 & 22 & 23 & 24 \\
\hline Overall $(\mathrm{kW})$ & 216.5 & 230.9 & 225.5 & 231.5 & 231.8 & 253.0 & 241.5 & 202.0 & 167.7 & 115.2 & 132.2 & 97.3 \\
\hline Kitchen $(\mathrm{kW})$ & 51.5 & 49.2 & 51.5 & 57.4 & 59.7 & 74.6 & 74.6 & 54.5 & 48.5 & 41.8 & 27.6 & 22.4 \\
\hline Constant $(\mathrm{kW})$ & 100 & 100 & 100 & 100 & 100 & 100 & 100 & 100 & 100 & 100 & 100 & 100 \\
\hline
\end{tabular}

Total load on micro-grid (kW)

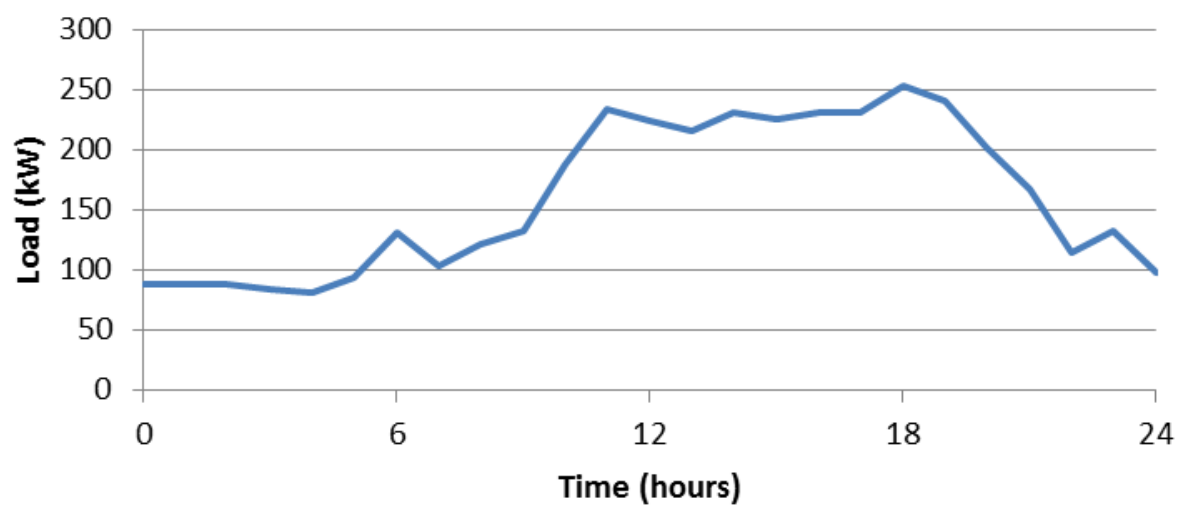

— Total load on micro-grid (kW)

Figure C-1. Load Profiles 
Inductive load was supplied by the load bank at the Caterpillar Tech Center. Load bank capacity is up to $778 \mathrm{kVA}$ and 0.7 power factor. The inductive load capability of the load bank was used to simulate the starting of multiple air conditioning units, which typically impose heavy inductive loads.

It was expected that weather conditions would affect the PV system output power. Testing and schedule were designed to accommodate a wide range of PV system output power. However, since the specific output power of the PV system was not a focus of this testing, weather conditions did not significantly impact testing.

\section{C.1.1 Test Phases}

The test plan divided the testing effort into three phases. The first phase, the verification phase, concentrated on testing the operation of the implemented microgrid to verify operation according to specifications. The second phase, the evaluation phase, concentrated on evaluating the microgrid's performance under various operating modes and loads. The third phase, the demonstration phase, was an opportunity to implement knowledge gained from the evaluation phase to tune and optimize the microgrid over a significant period (two days) to obtain practical data on best-case diesel or JP-8 fuel reduction. The test plan divided each testing phase into scenarios that focus on specific microgrid elements or the interactions of two or more elements.

\section{C.1.2 Data Collection}

The objective of data collection in the verification phase was to acquire sufficient data to ascertain whether the microgrid components and integrated system operated according to design specifications. The object of data collection in the evaluation phase was to acquire sufficient data to characterize and evaluate the performance of the microgrid and compare the performance of a basic microgrid to performance under SAGE operation.

Prior to testing, the following information was recorded for all equipment comprising the microgrid:

- manufacturer

- model

- serial number

- manufacturer catalog/specification sheets

- photographs of installed equipment (front, back, sides)

- manufacturer's installation drawings

Existing site data-logging equipment was used when available and compatible with the data to be acquired.

Specialized measurement equipment, such as power monitors and oscilloscopes, was used to assess power quality. Properties measured included voltage, current, and frequency, as well as 
waveform capture. Measurements were performed at key points on the microgrid, such as the battery system before and after the inverter and charger, generator outputs, and the PV system before and after microinverters. Measurement equipment recorded data over the duration of the test in a central comma-separated-variable file. Fuel consumption was measured continuously.

Issue reports were generated to log any anomalies observed during testing. All issue reports can be found in the original SAGE Phase 1 Test Report.

\section{Phase 2 Tests}

\section{C.1.3 Microgrid Testing}

The primary purpose of the microgrid testing was the establishment of fuel efficiency for both the microgrid and traditional spot generation and the fuel savings potential of the microgrid. The test period occurred on days with optimal solar insolation (that is, optimal PV production).

\section{C.1.3.1 Methodology}

Load profiles were programmed into a Simplex ${ }^{\circledR} 700$ load bank and separately run on the microgrid and the single TQG-60 generator. The scaled-down TIAX load profiles for individual facilities (billets, kitchen, laundry, latrine, and shower) were run for 24 hours each on a single TQG-60 generator to establish the baseline spot generation fuel efficiency numbers. For the microgrid, the load profiles were combined for one 24-hour run. This combined profile was run once without the PV array and again with the PV array to establish the PV's contribution to efficiency.

\section{C.1.3.2 Data Collection}

Electrical parameters were measured directly at the load bank by a calibrated three-phase power analyzer. Voltage, current, power (apparent, reactive, and real), power factor, and frequency were logged in 5-second intervals for the duration of each 24-hour test. Electrical energy was integrated in 5-minute intervals for the 24-hour period.

Fuel was pumped from one of two central fuel tanks to onboard tanks on each generator to a predetermined level prior to and after each test. A fuel-flow totalizing meter at the two central fuel tanks recorded the total quantity pumped.

\section{C.1.3.3 Parasitic Loads}

The Simplex 700 load bank dissipates its electrically generated heat using a fan that draws approximately $4.5 \mathrm{~kW}$. This fan would not exist in an actual Forward Operating Base, and the fan is thus a parasitic load that made the low-load spot generator efficiencies appear higher than if the load bank fan did not exist. In post-test data analysis, $4.5 \mathrm{~kW}$ was subtracted off every 5minute power value for every test, and the energy values were recomputed. To correct the "fuel consumed" numbers for the TQG-60 spot generator, a generator load curve was constructed for 
the generator from values obtained in testing and from Caterpillar specifications. Data from this testing and from Caterpillar were used for this calculation. The load-curve equation was then used to calculate generator efficiency at the slightly lower load levels when the parasitic load was subtracted. The resulting calculated fuel consumption was lower than the originally recorded numbers. The microgrid efficiency was not recalculated because the size of the parasitic load on the microgrid had far less impact than it had on the single TQG-60 generator.

Another small parasitic load remains on the microgrid profile from the power required to run the microgrid control system and the energy storage system's air conditioning unit. These loads were not subtracted because they are always required to run a microgrid.

\section{C.1.4 Solar Hot Water Testing}

The objective of the solar hot water (SHW) testing was to determine the energy that can be contributed by a SHW heating system, and to quantify the associated fuel savings of an auxiliary heater.

The Phase 2 tests were conducted over a 24-hour period on June 20, 2012, and October 11, 2012, according to a test procedure developed by Pacific Northwest National Laboratory (PNNL). The tests were conducted using both camps at the Base Camp Integration Laboratory (BCIL); the Baseline camp was on the south side of the BCIL and the SAGE camp (which housed the SHW system) was on the north side of the BCIL. The Baseline and SAGE camps had identical shower facilities; however, the hot water demand was supplied by an AWH-400 in the Baseline camp and by both the SHW system and an AWH-400 in the SAGE camp. The tests were conducted during the same time period in both the SAGE camp and the Baseline camp. A direct comparison between the camps was made.

\section{C.1.4.1 Methodology}

The SHW test was conducted over 24 hours to obtain a 24-hour power demand profile for the shower facilities. The 24 hour period included a 15 hour performance test on the SHW system per the PNNL-developed test procedure. The test procedure included a hot water demand load profile that was scaled off the consumption pattern of the shower TIAX profile. The test consisted of manually turning showers on and off to simulate 150 showers in each of the south (Baseline) and north (SAGE) camps. The demand profile was higher in the morning and evening than the rest of the day, which is a common domestic hot water load pattern. ${ }^{16}$ The procedure stated a typical domestic hot water preset target for shower water temperature $\left(107^{\circ} \mathrm{F}\right)$ and required preset temperatures on the auxiliary fuel-fired heater. Pretest training, checklists, and key parameters to measure were also included.

The procedure allowed the SHW system to store energy one day prior to the test; thus storage tank temperatures were measured before and after the test to quantify the stored energy available.

\footnotetext{
${ }^{16}$ Mutch, JJ. 1974. Residential Water Heating: Fuel Conservation, Economics, and Public Policy. R-1498-NSF, RAND Corp., Santa Monica, CA.
} 
The Phase 2 test results demonstrate how the SHW system responds to a fairly high, yet controlled, demand load profile.

Test operators reviewed the test procedures and performed final test preparations the day before the test. To facilitate testing, the showers were numbered sequentially in each camp. The showers combine cold ground water with hot water to produce tempered shower water. The water temperatures were measured by a calibrated thermometer, and the shower nozzle handles were set and marked to provide a target temperature of $\sim 107^{\circ} \mathrm{F}$. Likewise, the standard dieselfired water heaters (AWH-400 units) were set before the test to a target temperature of $\sim 120^{\circ} \mathrm{F}$ in June and $\sim 140^{\circ} \mathrm{F}^{17}$ in October. Thus, the AWH-400 units turned on whenever their input water temperature was lower than target temperature, and shut off shortly after reaching that target temperature. It was observed that there were slight differences in the operation of the two units, thus the factory settings were adjusted so that the two would operate similarly. The tests were implemented per the test procedure; however, a few problems did occur during testing, such as a shortage of water during the June test. Subsequently, corrective actions were taken and the problem was recorded on issue report forms.

\section{C.1.4.2 Data Collection}

The Baseline and SAGE camps had identical layouts with ample metering. A standard Army fuel-fired hot water heater (AWH-400) was in each camp. The AWH-400 units were supplied diesel fuel from two 55-gallon drums located near the unit. Only one drum supplied fuel to the unit at a time; thus, before each test the drum being used for the test was filled to the top. As a supplemental check, the fuel drums were measured with a dip stick before, after and periodically during the test. After the test, the drums were refilled and measured with an in-line flow meter.

Water temperatures were taken at the output and the intake to the AWH-400 units in each camp, and flow into the AWH-400 units was measured.

The water temperature supplied to the SHW system was measured, as well as potable water flow into the system. In addition, during the Phase 2 tests, water temperatures at the discharge of the shower nozzles were taken in each camp throughout the 15-hour test to make sure that the target water temperature was being met.

The SAGE SHW system was designed and built with sensors and meters that supplemented those in the camp. The SHW system had a variety of sensors for control, as well as temperatures in and out of the system, flow into the system and parasitic power into the system for monitoring performance as shown in Figure C-2 and Figure C-3.

\footnotetext{
${ }^{17}$ The supply water temperature was lower in October, so the target temperature on the AWH-400 was raised to be able to meet the $107^{\circ} \mathrm{F}$ target shower temperature.
} 


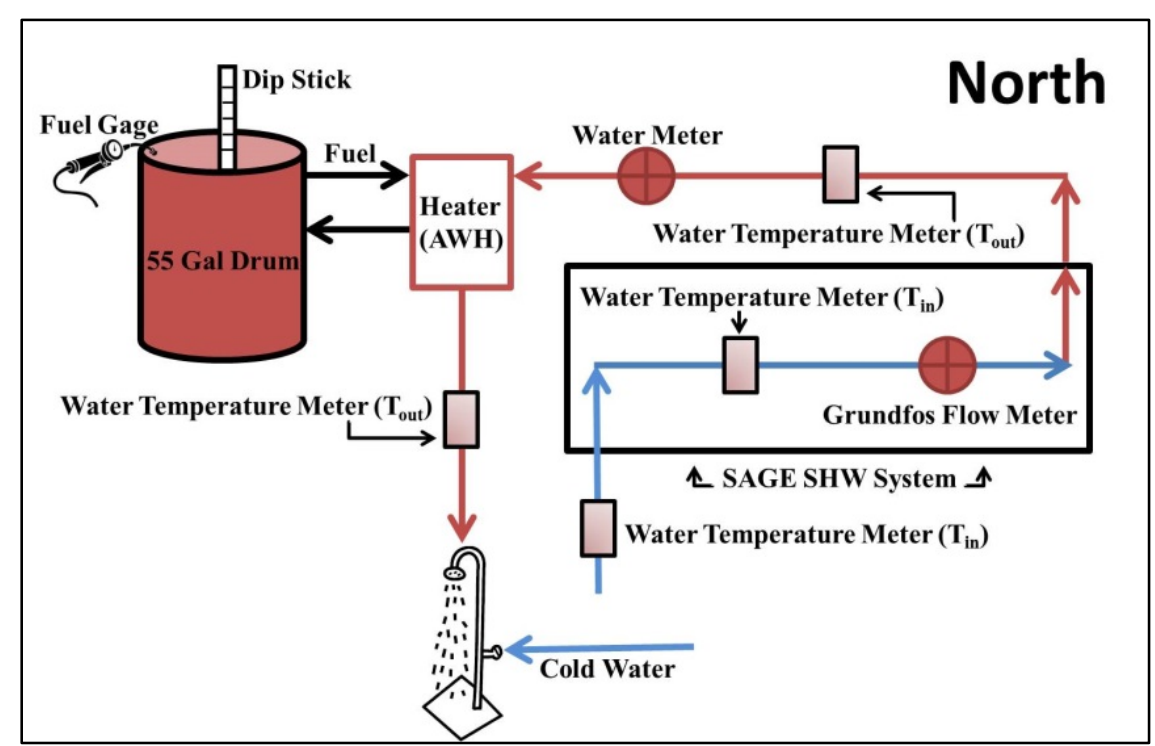

Figure C-2. North Camp Test Metering

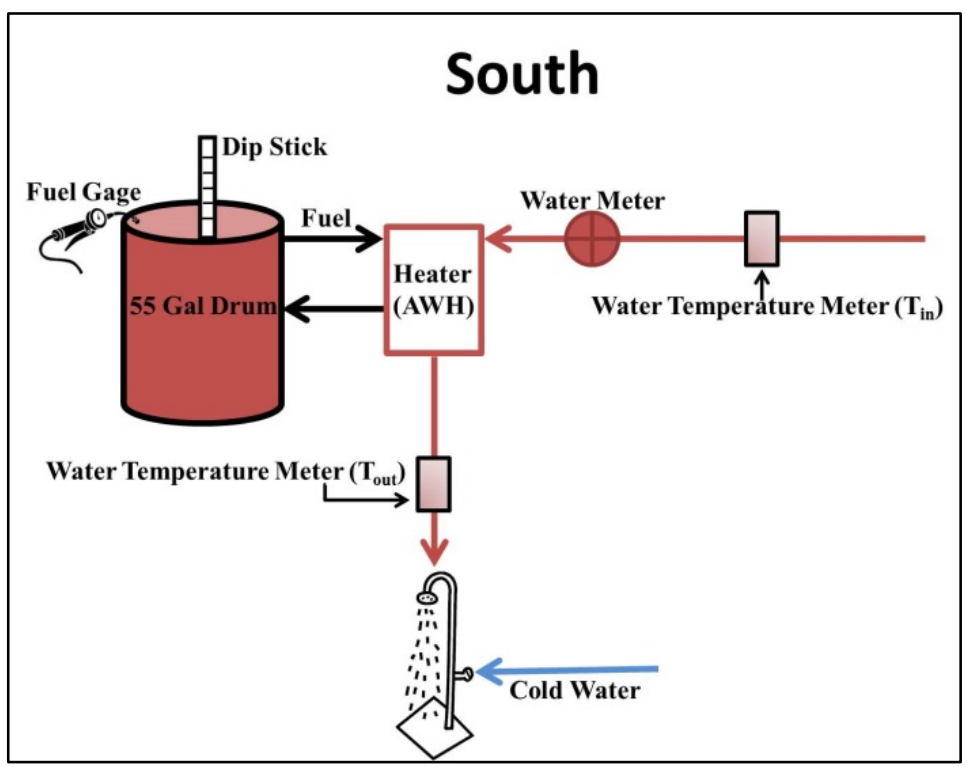

Figure C-3. South Camp Test Metering

\section{C.1.5 Energy Efficient Shelter Testing}

Testing of the SAGE energy efficient shelters with high efficiency heat pumps compared the energy consumption of a SAGE shelter against the standard Army air beam tent and its F100 heating/cooling unit (or environmental control units (ECUs)). Testing confirmed that the shelters consumed less energy than the tents. However, the BCIL temperature instrumentation inside some shelters and most tents, as well as the BCIL power instrumentation, performed unreliably, and SAGE considers the test results too unreliable for annual energy savings estimates. As such, this section only illustrates the testing methodology, the flawed results, and 
why these results were not used in further analysis. As a consequence, both tents and shelter structures were extensively modeled. This confirmed the savings found during testing.

\section{C.1.5.1 Methodology}

Each shelter and tent tested was set for either heating or cooling to a predetermined temperature set point, typically to cool to $65^{\circ} \mathrm{F}$ and to heat to $75^{\circ} \mathrm{F}$. In some billets, the cooling set point was varied and documented in field notes. Testing took place over a total period of two weeks. The internal temperatures and ongoing power levels were monitored during testing. After test conclusion, the power data was integrated to obtain energy consumption numbers for individual 24 hour periods for each structure. Weather data (heating degree days and cooling degree days) for the test period were then used to calculate a kilowatt-hour per cooling (or heating) degree-day figure.

\section{C.1.5.2 Data Collection}

Power data was collected remotely via Decision Support for Operations and Maintenance (DSOM) software from power meters installed at each structure. It was later integrated to obtain energy data.

DSOM attempted to collect temperature data from BCIL thermocouples. This data was frequently corrupted or missing altogether.

Weather data was collected via DSOM.

\section{C.1.6 DSOM Testing}

The goal of this test was to test DSOM's load shedding ability, remote breaker operation, and more to the point, the effectiveness of the tiered load shedding. Towards this goal, PNNL measured the various electrical shelter loads that can be controlled by DSOM. These included lights, plug loads, and the heating, ventilation and air conditioning (HVAC) system. A secondary goal was testing the remote controlled breakers and tiered load shedding.

\section{C.1.6.1 Methodology}

A power quality meter was used to record power quality for the tested shelter. The test started with every load (except the communication module for the breakers) turned off to determine a baseline power reading. Each load was energized individually via the corresponding breaker for a definite length of time, and then de-energized. The load levels were later determined by examining the power quality data.

\section{C.1.6.2 Data Collection}

The power quality meter was set up to record voltage, current, frequency, and power factor, all with definitive time stamps. At each load change, the time and load were recorded on a paper 
datasheet. The power quality data was later matched up with the recorded information on the datasheet to establish load levels.

\section{Phase 3 Tests}

\section{C.1.7 Overall 72-Hour Test Setup}

The 72-hour Phase 3 test held October 18, 2012, through October 21, 2012, utilized real troop occupancy in both the BCIL north camp (SAGE) and the south camp (Baseline). The north camp was home to 157 troops and used all SAGE technology integrated into one camp:

- microgrid

- energy storage system (batteries and bidirectional inverter)

- PV system (1/4 of full SAGE capacity)

- three energy efficient shelters with high efficiency mini-split heat pumps

- SHW system

\section{- DSOM}

The south camp was home to approximately 100 troops and used Baseline technology:

- six TQG-60 generators set up for spot generation

- standard AWH-400 heated showers

- tents with non-Baseline insulation liners

A direct side-by-side comparison of the Baseline and SAGE camps was not possible (without normalizing) due to these factors:

- uneven troop loading (157 vs. 100)

- use of non-Baseline insulation liners in south camp tents 


\section{Appendix D \\ Detailed Modeling and Annualization Methodology}

\section{Introduction}

This appendix to the U.S. Army Smart and Green Energy (SAGE) Final Report contains detailed information regarding the modeling and annualization methodology used in the SAGE project. A combination of field testing and sophisticated computer modeling resulted in credible predictions about energy footprints and fuel usage for bases of three sizes in fourteen different locations.

\section{D.1.1 Overview}

The field testing at the Base Camp Integration Laboratory (BCIL) facility at Fort Devens allowed the proof-of-concept for the variety of SAGE technologies under consideration:

- smart microgrid with load sharing technology

- energy storage system with bidirectional inverter

- renewable energy source (photo-voltaic array)

- solar hot water system

- insulated, hard-walled shelter with right-sized high-efficiency environmental control unit (ECU)

- base camp energy management system (Decision Support for Operation and Maintenance, or DSOM).

Performance data was collected for a baseline camp and a camp equipped with SAGE technology (SAGE camp). Data collection took place in a variety of seasonal conditions and with unoccupied as well as occupied camps. This data was used to calibrate software models of each structure in both camps. These calibrated models were then used to compute the annual energy and fuel needs for camps of three different sizes in fourteen different locations.

\section{D.1.2 Data Collection}

The test methodology of the field testing is found in Appendix C - Detailed Field Testing Methodology. The results of the field testing are found in Appendix E-Detailed Field Testing Results.

\section{D.1.3 Modeling}

The software used for all structure/building modeling was EnergyPlus, a whole building energy simulation program. The software models heating, cooling, lighting, ventilation, water use, and other energy flows. It provides time-dynamic modeling of buildings based on usage patterns for the short term and daily and seasonal climate patters for the long term. The software 
can also model photovoltaic renewable energy installations, a feature which was utilized for SAGE modeling.

\section{Climate Zones}

The U.S. Army Logistics Innovation Agency (LIA) selected the following 14 bases to represent the major FOB climate zones around the world.

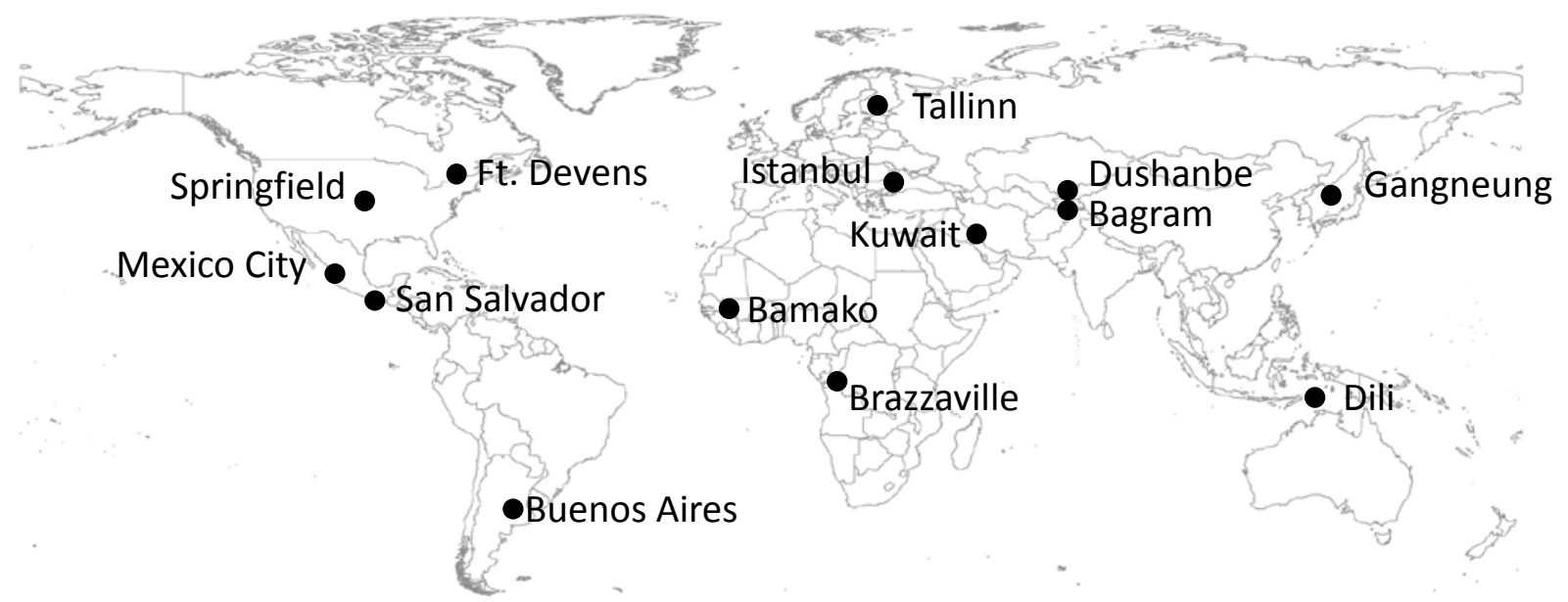

SAGE modeling assigned each base to one of the 19 climate zones developed by ASHRAE for more clarity on how climate may relate to fuel savings. The assignment correlated the climate data from the nearest weather station to each base to the ASHRAE zones. The weather station data related for one base, Kabul, Afghanistan, put the base into a different zone than indicated by the ASHRAE map. All of the selected bases fell into just 7 of the 19 climate zones. 
The 19 climate zones are:

\begin{tabular}{|c|l|l|}
\hline Zone with Base & Zone & Description \\
\hline $\mathrm{X}$ & Zone 0A & Extremely Hot Humid \\
\hline & Zone 0B & Extremely Hot Dry \\
\hline $\mathrm{X}$ & Zone 1A & Very Hot Humid \\
\hline & Zone 1B & Very Hot Dry \\
\hline & Zone 2A & Hot Humid \\
\hline $\mathrm{x}$ & Zone 2B & Hot Dry \\
\hline & Zone 3A & Warm Humid \\
\hline $\mathrm{x}$ & Zone 3B & Warm Dry \\
\hline $\mathrm{x}$ & Zone 3C & Warm Marine \\
\hline & Zone 4A & Mixed Humid \\
\hline $\mathrm{x}$ & Zone 4B & Mixed Dry \\
\hline & Zone 4C & Mixed Marine \\
\hline & Zone 5A & Cool Humid \\
\hline & Zone 5B & Cool Dry \\
\hline $\mathrm{x}$ & Zone 5C & Cool Marine \\
\hline & Zone 6A & Cold Humid \\
\hline & Zone 6B & Cold Dry \\
\hline & Zone 7 & Very Cold \\
& Zone 8 & Subarctic \\
\hline & & \\
\hline
\end{tabular}

The ASHRAE zone map:

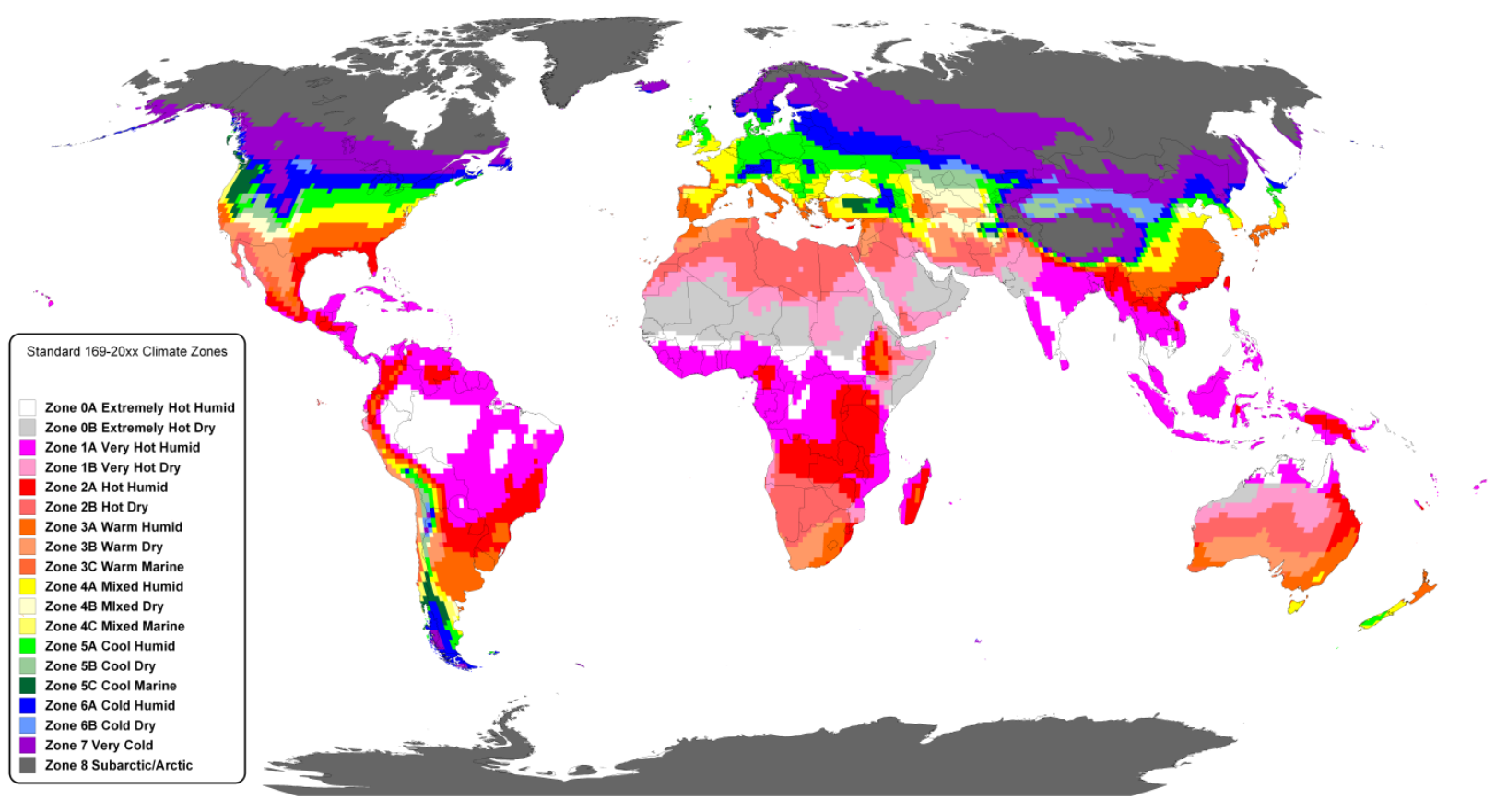


The Climate Zones for Each Base

\begin{tabular}{lll}
\hline DOD Command & Base & ASHRAE Zone Name \\
\hline Africa & Bamako & Zone 1B Very Hot Dry \\
& Brazzaville & Zone 1B Very Hot Dry \\
Central & Dushanbe & Zone 4A Mixed Humid \\
& Kabul (Bagram) & Zone 4A Mixed Humid \\
& Kuwait City & Zone 1B Very Hot Dry \\
Europe & Istanbul & Zone 3A Warm Humid \\
& Tallinn & Zone 6A Cold Humid \\
North & Fort Devens, Worcester, MA & Zone 5A Cool Humid \\
& Mexico City & Zone 3C Warm Marine \\
& Springfield, MO & Zone 4A Mixed Humid \\
Pacific & Dili & Zone 0A Extremely Hot Humid \\
& Gangneung & Zone 4A Mixed Humid \\
South & Buenos Aires & Zone 3C Warm Marine \\
& San Salvador & Zone 0A Extremely Hot Humid \\
\hline
\end{tabular}

DOD = U.S. Department of Defense

The Bases Sorted By Climate Zone

\begin{tabular}{lll}
\hline ASHRAE Zone & DOD Command & Base \\
\hline Zone 0A Extremely Hot Humid & Pacific & Dili \\
& South & San Salvador \\
Zone 1B Very Hot Dry & Africa & Bamako \\
& & Brazzaville \\
& Central & Kuwait City \\
Zone 3A Warm Humid & Europe & Istanbul \\
Zone 3C Warm Marine & North & Mexico City \\
& South & Buenos Aires \\
Zone 4A Mixed Humid & Central & Dushanbe \\
& & Kabul (Bagram) \\
& North & Springfield, MO \\
& Pacific & Gangneung \\
Zone 5A Cool Humid & North & Fort Devens, Worcester, MA \\
Zone 6A Cold Humid & Europe & Tallinn \\
\hline
\end{tabular}

\section{Base Details}

\section{D.1.4 5000-Soldier Base}

The key characteristics of the 5000-Soldier base were approximately 400 wood framed buildings, totaling 1.4 million $\mathrm{ft}^{2}$. Almost all buildings use $2 \times 4$ walls, and R-19 batt insulation 
was used in all exterior walls. One $3 \mathrm{MW}$ generator was stationed in each of the base's nine sectors; generators were not connected to each other, which is commonly referred to as spot generation. LIA's key information included the following from the Theater Construction Management System (TCMS):

- Overall civil and electrical site plans for a 5000-Soldier base. The drawing title: Contingency Standard Designs, Army Facilities Components System (AFCS), AFCS Installation, 5000-Solider Base Camp, Site Plan, Temporary Standard. Drawing number NT3001-01, 6-25-2013.

- Materials and equipment inventory (Bill of Materials (BOM)) for procuring base construction packages and materials. The list is titled Super Component BOM, By Component, By Facility, By Construction, and Sequence Super Component: BCWD5000 Base Camp, 5000 Man, Temporary Standard, Brigade Combat Team, Wood.

- Drawings and BOMs for wood framed buildings, including the following building types: Administration, Barracks, Medical (TMC), Dental, Warehouse, Dining (with a full service kitchen), Gymnasium, and Vehicle Maintenance.

\section{D.1.5 Characterization}

SAGE characterized each building (building, container building, and tent) within each of the following factors that can have a major effect on energy use:

- building square footage

- building occupancy or use

- building occupancy hours

- building occupant density

- building construction materials

- building plug loads

- building site climate

Solar orientation was not included because, upon inspection of drawings and photos of existing bases, a base may be oriented around any compass direction, and the base's buildings may also vary from the overall base's grid.

A building's footprint or size in square feet $\left(\mathrm{ft}^{2}\right)$ is used to estimate several energy use factors, including plug loads, quantity of occupants, and heating and cooling loads. All of buildings in the 150-, 600-, and 5000-Soldier bases are a simple rectangle shape.

SAGE classified each building into one of the following building size groups that were defined to follow the major size breaks in the 5000-Soldier base:

- buildings less than $1200 \mathrm{ft}^{2}$ 
- buildings 1,200 $\mathrm{ft}^{2}$ or larger

- buildings 2,000 $\mathrm{ft}^{2}$ or larger

- buildings $6,000 \mathrm{ft}^{2}$ or larger

- buildings $17,000 \mathrm{ft}^{2}$ or larger

- buildings $45,000 \mathrm{ft}^{2}$ or larger

\begin{tabular}{|c|c|c|c|c|}
\hline Size Group & Building $\mathrm{ft}^{2}$ & Occupancy Type & $\begin{array}{l}\text { Building } \\
\text { Quantity }\end{array}$ & Total $\mathbf{f t}^{2}$ \\
\hline \multirow[t]{5}{*}{ 1. $>1200 \mathrm{ft}^{2}$} & & & 105 & 73,433 \\
\hline & 121 & Special & 1 & 121 \\
\hline & 160 & Bathroom & 15 & 2,400 \\
\hline & 512 & Office & 1 & 512 \\
\hline & 800 & Bathroom & 88 & 70,400 \\
\hline \multirow[t]{4}{*}{ 2. $1200+\mathrm{ft}^{2}$} & & & 59 & 71,664 \\
\hline & 1,200 & Office & 57 & 68,400 \\
\hline & 1,600 & Office & 1 & 1,600 \\
\hline & 1,664 & Office & 1 & 1,664 \\
\hline \multirow[t]{7}{*}{ 3. $2000+\mathrm{ft}^{2}$} & & & 85 & 227,000 \\
\hline & 2,000 & Office & 60 & 120,000 \\
\hline & & Warehouse & 1 & 2,000 \\
\hline & 3,000 & Office & 2 & 6,000 \\
\hline & 4,500 & Assembly & 5 & 22,500 \\
\hline & & Office & 16 & 72,000 \\
\hline & & Warehouse & 1 & 4,500 \\
\hline \multirow[t]{7}{*}{ 4. $6000+\mathrm{ft}^{2}$} & & & 104 & 624,000 \\
\hline & 6,000 & Assembly & 2 & 12,000 \\
\hline & & Housing & 89 & 534,000 \\
\hline & & Kitch \& Dining & 6 & 36,000 \\
\hline & & Medical & 2 & 12,000 \\
\hline & & Office & 4 & 24,000 \\
\hline & & Warehouse & 1 & 6,000 \\
\hline \multirow[t]{3}{*}{ 5. $17,000+\mathrm{ft}^{2}$} & & & 15 & 253,376 \\
\hline & 10,000 & Warehouse & 1 & 10,000 \\
\hline & 17,384 & Shop & 14 & 243,376 \\
\hline \multirow[t]{3}{*}{ 6. $45,000+\mathrm{ft}^{2}$} & & & 4 & 195,000 \\
\hline & 45,000 & Gym & 1 & 45,000 \\
\hline & 50,000 & Warehouse & 3 & 150,000 \\
\hline Total & & & 372 & $1,444,473$ \\
\hline
\end{tabular}


For the 150- and 600-Soldier bases, the base buildings sizes were either a $20 \times 32$ tent of $640 \mathrm{ft}^{2}$, or a container-building, called a Tricon, which was $6.5^{\prime}$ wide by 8 ', 13', or $18^{\prime}$ long (52 $\mathrm{ft}^{2}, 85 \mathrm{ft}^{2}$, or $120 \mathrm{ft}^{2}$, respectively). The distribution of buildings per grouping is shown in the following table:

\begin{tabular}{|c|c|c|c|c|c|c|}
\hline \multirow[b]{2}{*}{ Size Groups } & \multirow[b]{2}{*}{$\mathbf{f t}^{2}$ Each } & \multirow{2}{*}{$\begin{array}{c}\text { Occupancy } \\
\text { Type } \\
\end{array}$} & \multicolumn{2}{|c|}{150} & \multicolumn{2}{|c|}{600} \\
\hline & & & Quantity & Total $\mathbf{f t}^{2}$ & Quantity & Total $\mathbf{f t}^{2}$ \\
\hline \multirow[t]{6}{*}{ Container Sizes } & & & 11 & 1,073 & 22 & 2,442 \\
\hline & 52 & Special & 1 & 52 & 4 & 208 \\
\hline & 85 & Special & 5 & 423 & & \\
\hline & 120 & Bathroom & 4 & 478 & 16 & 1,914 \\
\hline & & Kitchen & 1 & 120 & & \\
\hline & 160 & Kitchen & & & 2 & 320 \\
\hline \multirow[t]{8}{*}{ Tent Size } & & & 11 & 7,040 & 78 & 49,920 \\
\hline & 640 & Assembly & & & 7 & 4,480 \\
\hline & & Dining & 1 & 640 & 5 & 3,200 \\
\hline & & Gym & & & 1 & 640 \\
\hline & & Housing & 10 & 6,400 & 40 & 25,600 \\
\hline & & Medical & & & 3 & 1,920 \\
\hline & & Office & & & 14 & 8,960 \\
\hline & & Shop & & & 8 & 5,120 \\
\hline Total & & & 22 & 8,113 & 100 & 52,362 \\
\hline
\end{tabular}

\section{D.1.6 Occupancy/Use Factor}

Each building was classified into one of the following use groups. These uses affect the estimated occupancy patterns (high quantity versus low quantity of occupants), throughout a day.

- assembly

- bathroom

- dining-only

- kitchen-only

- dining and kitchen

- gymnasium

- housing

- medical

- office

- shop 
- special

- warehouse

The Occupancy Hours Factor is the operation hours, with the quantity of people using the space throughout a day. This is assumed to be the same pattern for each day of the week. This factor addresses how base operations differ from commercial operations. Modeling assumptions associated with the Occupancy Hours Factor include:

- 24 hours a day, 7 days a week (e.g., command centers, medical, etc.)

- 4 a.m.-11 p.m. (kitchens)

- 6 a.m. - 11 p.m. (long day for office, admin, and dining functions)

- Residential (low occupancy during the day, high at night)

- Occupant Density

The Construction Materials Factors include envelope, insulation, heating and cooling use, and lighting options. The key modeling assumptions include:

- Envelope materials

$\circ$ Wood framing (for the 5000-Soldier base; almost all walls are $2 \times 4$ )

$\circ$ Tent fabric (for most of the buildings in the 150- and 600-Soldier bases)

- Container (a steel shipping container adapted to house kitchens and showers; a minority of the buildings in the 150- and 600-Soldier bases)

- Insulation

- R-19 batt insulation in a $2 \times 4$ cavity (5000-Soldier base; a few buildings use $2 \times 6$ studs)

- Tent fabric (very low insulation)

- Container (low insulation)

- Heating/Cooling

○ Heated and cooled (most buildings)

- No Heating or Cooling (warehouses)

- Lighting

- Only a single group was used for lighting: commercial fluorescents of T12 and electronic ballasts 
The Plug Loads Factor represents all equipment plugged into wall outlets. The Plug Load Factor was binned into three categories: low, medium, and high.

- low (e.g., warehouses, dining space)

- medium (e.g., tents, admin)

- high (e.g., command centers, medical, kitchens, shops, vehicle maintenance)

\section{Modeling Assumption Example for Tent and SAGE Shelter}

This section documents the modeling assumptions and scheduled operation hours for both the SAGE shelter and the baseline tent.

\begin{tabular}{|c|c|c|c|}
\hline \multicolumn{2}{|c|}{ Case } & \multicolumn{2}{|c|}{ Model 3} \\
\hline \multirow{2}{*}{\multicolumn{2}{|c|}{ Description }} & \multicolumn{2}{|c|}{ As Operational } \\
\hline & & Tent & Shelter \\
\hline \multirow{4}{*}{$\begin{array}{l}\text { Internal } \\
\text { Loads }\end{array}$} & Plug Loads & $1.56 \mathrm{~W} / \mathrm{ft}^{2}$ & $1.56 \mathrm{~W} / \mathrm{ft}^{2}$ \\
\hline & & $\begin{array}{l}\text { Without Plug Loads reduction } \\
\text { (Always On) }\end{array}$ & With Plug Loads reduction \\
\hline & People & 22 Occupants & 22 Occupants \\
\hline & Lighting & $\begin{array}{l}8-\mathrm{T} 12 \text { Fixtures } \\
320 \mathrm{~W} \text { or } 0.5 \mathrm{~W} / \mathrm{ft}^{2}\end{array}$ & $\begin{array}{l}8-\mathrm{T} 12 \text { Fixtures } \\
320 \mathrm{~W} \text { or } 0.5 \mathrm{~W} / \mathrm{ft}^{2}\end{array}$ \\
\hline \multirow[t]{3}{*}{ Heating } & Equipment & ECU & Heat Pump \\
\hline & & $\begin{array}{l}10 \mathrm{~kW} \text { Electric Resistance + } \\
\text { Supplementary Oil Furnace }\end{array}$ & HSPF 10 \\
\hline & $\begin{array}{l}\text { Schedule of } \\
\text { Operation }\end{array}$ & $\begin{array}{l}\text { ECU - } 1 \text { April to } 31 \text { October } \\
\text { Oil Furnace - } 1 \text { November to } \\
31 \text { March }\end{array}$ & All Year \\
\hline \multirow[t]{4}{*}{ Cooling } & Equipment & $\mathrm{ECU}$ & Heat Pump \\
\hline & & $\begin{array}{l}\text { DX Cooling, } 5 \text { Ton Capacity } \\
\text { COP } 3.2\end{array}$ & SEER 19 \\
\hline & Schedule of & ECU - 1 April to 31 October & \\
\hline & Operation & $\begin{array}{l}\text { No Cooling - } 1 \text { November to } \\
31 \text { March }\end{array}$ & \\
\hline \multirow[t]{4}{*}{ Setpoints } & Heating & Heating $-72^{\circ} \mathrm{F}$ & Heating $-72^{\circ} \mathrm{F}$ \\
\hline & & No Setbacks & $\begin{array}{l}3^{\circ} \mathrm{F} \text { Setback (From } 6 \text { a.m. to } \\
10 \text { p.m.) }\end{array}$ \\
\hline & Cooling & Cooling $-75^{\circ} \mathrm{F}$ & Cooling $-75^{\circ} \mathrm{F}$ \\
\hline & & No Setbacks & $\begin{array}{l}3^{\circ} \mathrm{F} \text { Setback (From } 6 \text { a.m. to } \\
10 \text { p.m.) }\end{array}$ \\
\hline
\end{tabular}




\section{Assumptions for Energy Model}

\section{D.1.7 Weather file}

a. Worchester, Massachusetts (No weather file available for Devens or Leominster)

b. TMY $3^{18}$ Weather file

\section{D.1.8 Dimensions $-32^{\prime} \times 20^{\prime} \times 9^{\prime}$}

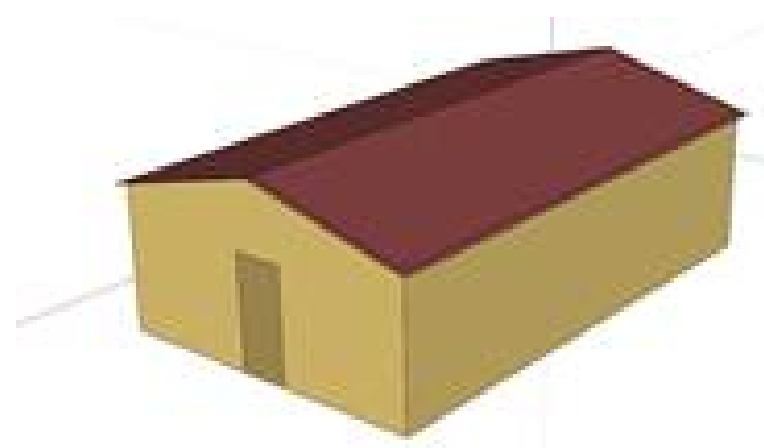

Shelter as Modeled

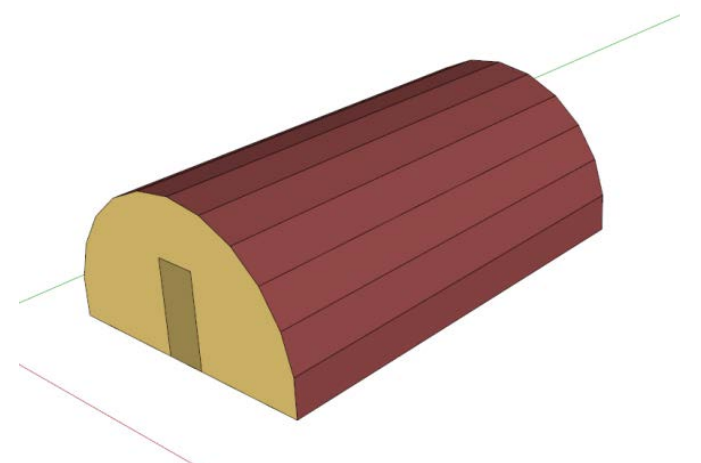

Tent as Modeled

\section{D.1.9 Construction}

a. Shelter - R-25 panels are used for exterior walls, roof, doors.

b. Tent - R-1 panels used for wall, roof, doors. Floor has been modeled with the same fabric and a 2" gypsum board below. No insulated board has been added for floor.

\section{D.1.10 Infiltration}

a. Shelter - Assumed tight construction. No Infiltration has been modeled.

b. Tent - Assumed infiltration level of $\sim 5 \mathrm{ACH} 50^{19}$

${ }^{18}$ TMY3 = typical meteorological year data, third version

${ }^{19} \mathrm{ACH} 50=$ air changes per hour under 50 pascals of pressure; CFM50 = cubic feet per minute at 50 pascals 
http://www.nebb.org/assets/1/7/2012_NEBB_Envelope_Testing_Presentation.pdf specifies the highest infiltration to be $\sim 0.40$ CFM50/ $\mathrm{ft}^{2}$ which corresponds to $\sim 3$ ACH50. However, 3 ACH50 is code minimum for residential buildings in climate zones 3-8. 5 ACH50 might represent a more leaky building.

\section{D.1.11 Temperature Setpoints}

a. For Calibration

i. Heating Setpoint

- Setpoint $65^{\circ} \mathrm{F}^{20}$

ii. Cooling Setpoint

- Setpoint $65^{\circ} \mathrm{F}$

b. As Designed

i. Heating Setpoint

- Setpoint $72^{\circ} \mathrm{F}$

- No Setbacks have been modeled for the Tent

- $3^{\circ} \mathrm{F}$ setback for Shelter

ii. Cooling Setpoint

- Setpoint $75^{\circ} \mathrm{F}$

- No Setbacks have been modeled for the Tent

- $3^{\circ} \mathrm{F}$ setback for Shelter

\section{D.1.12 Lighting Load}

a. $144 \mathrm{~W}$ lighting level assigned on a schedule for the shelter (all LED lights)

b. 8-40 W T-12 assumed for the Tent. Total connected W=320 W

\section{D.1.13 Plug Loads}

a. Tent
i. $1.56 \mathrm{~W} / \mathrm{ft}^{2}$

b. Shelter

i. $1.56 \mathrm{~W} / \mathrm{ft}^{2}$ assigned at a $100 \%$ schedule usage from 6 a.m. -10 p.m.

ii. Reduced to $50 \%$ usage from 10 p.m. -6 a.m., through the DSOM load management system.

\footnotetext{
${ }^{20}$ Energy Use has been measured at $75^{\circ} \mathrm{F}$ for heating. However, this results in simultaneous heating and cooling in the energy model and hence has not been simulated.
} 


\section{D.1.14 Occupants}

a. 22 Occupants have been modeled

\section{D.1.15 Tent Cooling Equipment - HDT F100-60K ECU}

a. DX Cooling

i. Rated Total Cooling Capacity - 58,000 Btu/hr

ii. Rated Sensible Ratio - 0.67. Not used. Has been autosized in EnergyPlus ${ }^{21}$

iii. Copeland Scroll Compressor Efficiency for R410 refrigerant = 3.2.

b. Electric Resistance Heating

i. Rated Heating Capacity - $10 \mathrm{~kW}$

c. Fan

i. Air Flow - $1900 \mathrm{cfm}$

ii. Static Pressure rise -2 " of water

iii. Fan efficiency $-53.5 \%$ (assumption)

iv. Motor Efficiency - 84\% (assumption)

d. Schedule of Operation

i. 1 April to 31 October

\section{D.1.16 Tent Zone Heater: MTH 150.}

a. Diesel Heater - MTH 150

b. Simulation Strategy

i. Modeled as a gas heater with reduced efficiency (EnergyPlus does not have the capability to model an oil heater).

ii. Reduced efficiency simulated in accordance with Lawrence Berkeley National Laboratory paper. $^{22}$ The thermal efficiency of gas furnace $(80 \%)$ has been modified to emulate an oil furnace $(67 \%)$.

c. Capacity $-130,000 \mathrm{Btu} / \mathrm{hr}, 38100 \mathrm{~W}$

d. Air flow - $1100 \mathrm{cfm}, 0.51 \mathrm{CMS}$

e. Schedule of Operation - 1 November to 30 March for Fort Devens.

\section{D.1.17 Mitsubishi MSY-GEO18 Heat Pump}

a. Rated Cooling Capacity - 17,200 Btu/hr

${ }^{21}$ EnergyPlus can autosize SHR, System Capacity, Airflow rate. Two of the three variables need to be autosized or ${ }_{22}$ it gives an error. Hence, the capacity has been hardsized. SHR and Airflow rate have been autosized.

http://efficiency.lbl.gov/drupal.files/ees/Simulating\%20a\%20Nationally\%20Representative\%20Housing\%20Sample \%20Using\%20EnergyPlus LBNL-4220E.pdf 
b. Rated Heating Capacity - 21,600 Btu/hr

c. From Specifications, $\mathrm{SEER}=19.2, \mathrm{HSPF}=10$

\section{D.1.18 Shelter - Broan HRV 90H}

a. Supply, Exhaust Fan

i. Static Pressure Rise - 0.4 in water $(100 \mathrm{~Pa})$ - From specifications

ii. Maximum flow rate- $96 \mathrm{cfm}-$ From specifications

iii. Motor efficiency- 0.9 (assumption)

iv. Supply fan efficiency - 0.8 (assumption)

v. Exhaust fan efficiency -0.5 (assumption)

b. Sensible, Latent Effectiveness for Heat Exchanger-

i. Sensible, latent effectiveness while heating- Taken from specifications

ii. Sensible, latent effectiveness while cooling not provided in specifications. Assumed 0.7 .

Plug Loads Calculations

\begin{tabular}{lcccccc}
\hline \multicolumn{1}{c}{ Equipment } & Quantity & $\begin{array}{c}\text { Typical } \\
\text { Hours/Day }\end{array}$ & Wattage & $\begin{array}{c}\text { Total } \\
\text { Wattage }\end{array}$ & $\begin{array}{c}\text { Monthly } \\
\text { kWh }\end{array}$ & $\begin{array}{c}\text { Yearly } \\
\text { kWh }\end{array}$ \\
\hline Laptops & 11 & 7 & 20 & 220 & 46.2 & 554.4 \\
Laser Printer & 2 & 4 & 80 & 160 & 19.2 & 230.4 \\
Copier & 1 & 4 & 183 & 183 & 21.96 & 263.52 \\
Fax & 1 & 4 & 15 & 15 & 1.8 & 21.6 \\
Coffee Maker & 2 & 3 & 900 & 1800 & 162 & 1944 \\
Desk Lamp & 11 & 3 & 75 & 825 & 74.25 & 891 \\
Microwave & 2 & 1 & 1000 & 2000 & 60 & 720 \\
Small Fridge (2.5-6.4 cu.ft) & 2 & 24 & 125 & 250 & 180 & 2160 \\
LCD Television & 2 & 2 & 70 & 140 & 8.4 & 100.8 \\
Miscellaneous & 4 & 4 & 66 & 264 & 31.68 & 380.16 \\
Peak Power Consumption & & & $\mathbf{2 5 3 4}$ & $\mathbf{5 8 5 7}$ & & \\
Annual Plug Loads Energy & & & & & & \\
Consumption & & & & & &
\end{tabular}

LCD = liquid crystal display 


\section{Heat Pump COP Calculations}

\begin{tabular}{|c|c|c|c|c|}
\hline & SEER & 19 & & \\
\hline 1 & EER & $\begin{array}{l}\left(-0.0182 \times(\mathrm{SEER})^{2}\right)+(1.1088 \times \\
\text { SEER })\end{array}$ & 14.5 & \\
\hline 2 & COP (w/o Fan Power Adjustment) & $\mathrm{EER} / 3.413$ & 4.25 & \\
\hline 3 & Unit Power Per ton & $12000 / \mathrm{COP} * 0.2937$ & 829.74 & $\mathrm{~W}$ \\
\hline 4 & $\mathrm{R}$ & $\begin{array}{l}\text { Fan Power Per Ton/Unit Power } \\
\text { Per Ton }\end{array}$ & 0.135 & \\
\hline 5 & COP (with fan power adjustment) & $(\mathrm{COP}+\mathrm{R}) /(1-\mathrm{R})$ & 5.066 & \\
\hline \multicolumn{5}{|c|}{ b. HSPF } \\
\hline & HSPF & 10 & & \\
\hline 1 & COP (w/o Fan Power Adjustment) & $\begin{array}{l}(-0.0255 \times(\mathrm{HSPF}) 2)+(0.6239 * \\
\text { HSPF })\end{array}$ & 3.69 & \\
\hline 2 & Unit Power Per ton & $12000 / \mathrm{COP} * 0.2937$ & 955.38 & $\mathrm{~W}$ \\
\hline \multirow[t]{2}{*}{3} & $\mathrm{R}$ & $\begin{array}{l}\text { Fan Power Per Ton/Unit Power Per } \\
\text { Ton }\end{array}$ & 0.12 & \\
\hline & COP (with fan power adjustment) & $(\mathrm{COP}-\mathrm{R}) /(1-\mathrm{R})$ & 4.05 & \\
\hline
\end{tabular}


Schedules of Operation

\begin{tabular}{lccc}
\hline & $\begin{array}{c}\text { Lighting Schedule } \\
\text { Fraction of Max Load }\end{array}$ & $\begin{array}{c}\text { Plug Loads } \\
\text { Occupancy Schedule } \\
\text { Fraction of Max Load }\end{array}$ & $\begin{array}{c}\text { Fraction of Max } \\
\text { Use }\end{array}$ \\
\hline Hour 1 & 0.07 & 1.00 & 0.5 \\
Hour 2 & 0.07 & 1.00 & 0.5 \\
Hour 3 & 0.07 & 1.00 & 0.5 \\
Hour 4 & 0.07 & 1.00 & 0.5 \\
Hour 5 & 0.19 & 1.00 & 0.5 \\
Hour 6 & 0.39 & 1.00 & 0.5 \\
Hour 7 & 0.44 & 1.00 & 1 \\
Hour 8 & 0.39 & 0.85 & 1 \\
Hour 9 & 0.17 & 0.39 & 1 \\
Hour 10 & 0.12 & 0.25 & 1 \\
Hour 11 & 0.12 & 0.25 & 1 \\
Hour 12 & 0.12 & 0.25 & 1 \\
Hour 13 & 0.12 & 0.25 & 1 \\
Hour 14 & 0.12 & 0.25 & 1 \\
Hour 15 & 0.12 & 0.25 & 1 \\
Hour 16 & 0.21 & 0.25 & 1 \\
Hour 17 & 0.44 & 0.30 & 1 \\
Hour 18 & 0.62 & 0.52 & 1 \\
Hour 19 & 0.83 & 0.87 & 1 \\
Hour 20 & 0.99 & 0.87 & 1 \\
Hour 21 & 1.00 & 0.87 & 0.5 \\
Hour 22 & 0.69 & 1.00 & 1 \\
Hour 23 & 0.38 & 1.00 & \\
Hour 24 & 0.16 & 1.00 & 1 \\
\hline
\end{tabular}

\section{Calibration of Models with Field Data}

\section{D.1.19 Field Data vs Initial Models}

Some of the initial uncalibrated models indicated significant differences between modeled load profiles and load profiles from measured occupied testing. Figure D-1 illustrates that the initially modeled electric power for a typical tent's lights and plug loads was higher than data collected from two tents during occupied testing indicated (in November 2012). 


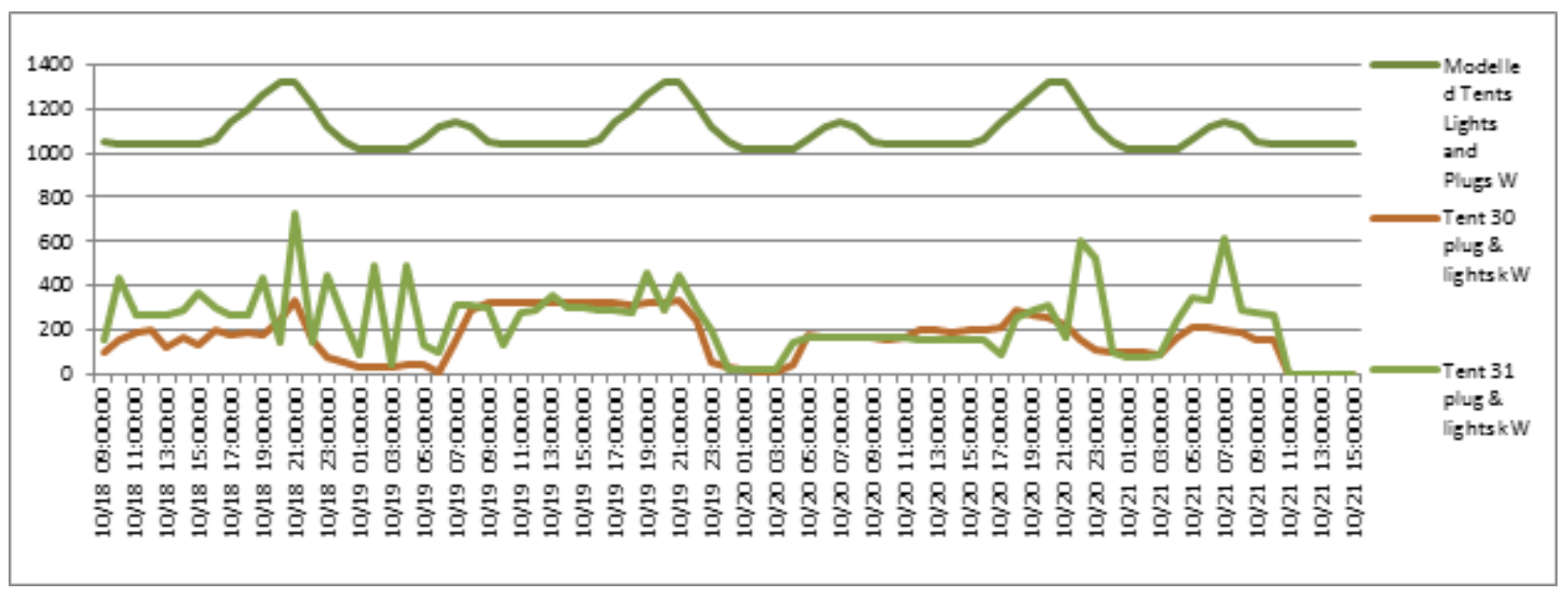

Figure D-1. Tent 30 and 31 Lights and Plug Loads vs Modeled Lights And Plug Loads

Figure D-2 shows that the average total load profile for four different tents was significantly different from a modeled profile (unoccupied testing in May 2012). It remained relatively flat over time, without large daily swings that the initial model predicted.

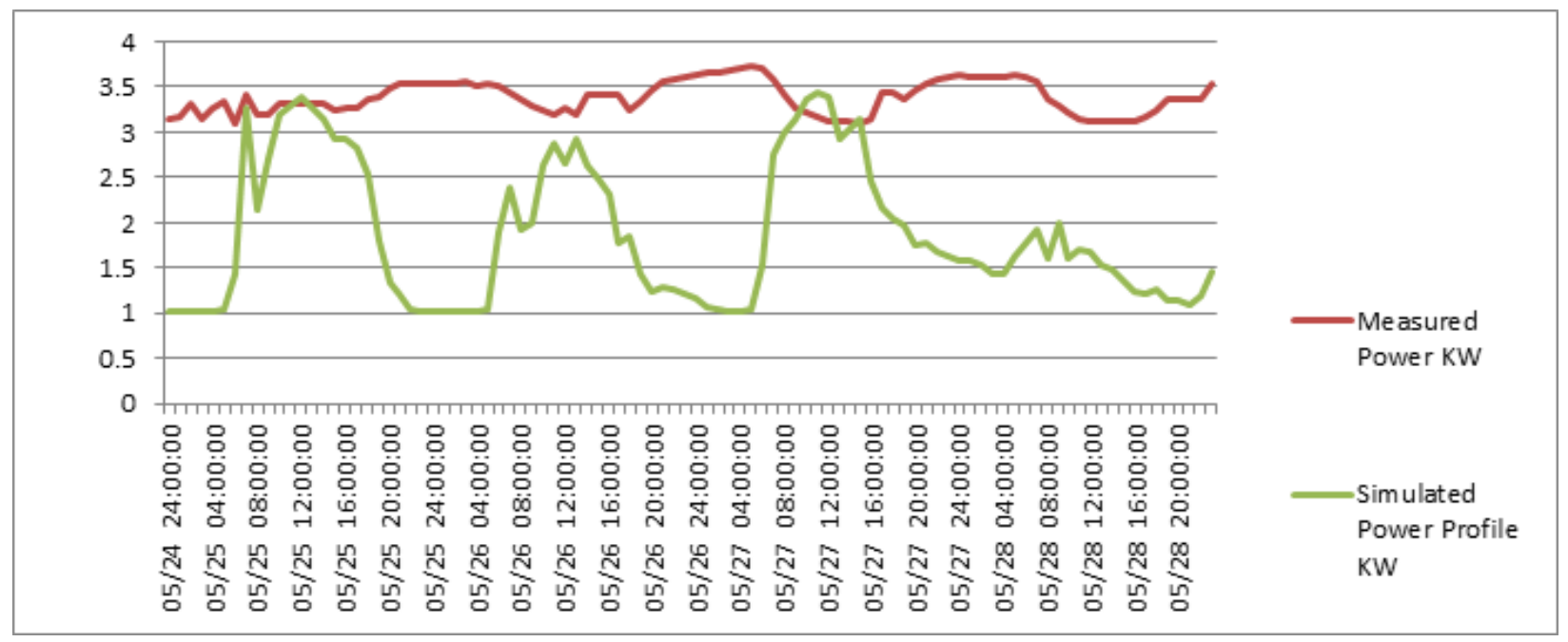

Figure D-2. Average Tent Profile vs Modeled Profile (unoccupied)

\section{D.1.20 Reasons for Discrepancies}

The large differences in modeled plug loads vs actual field plug loads is best explained in the nature of the SAGE camp at BCIL versus a "real" 150-Soldier camp. Troops were scheduled to stay at the SAGE camp for time periods of 4-10 days. As a consequence, many soldiers brought with them very few personal plug loads that would otherwise be present in an actual camp. This would include televisions, printers, personal computers, coffee pots, and small refrigerators loads that were confirmed during separate data collection efforts in Kuwait. As such, the modeled plug loads were not changed to match field data. Instead, field data was deemed to under-represent actual field conditions. 
The differences in overall tent load profiles to initial model profiles required careful calibration of the EnergyPlus models. The lack of pronounced daily variation in the load profile of a tent suggested the insulation and ECU performance were poorer than expected.

\section{D.1.21 Model Calibration}

The tent and shelter models were calibrated to more closely match the field data collected during unoccupied (Phase 2) testing at Fort Devens. The result of the first adjustment is shown in Figure D-3. Daily load profile variation is reduced, but it is still not as flat as the field data. The model shows a reduction in power use at night time hours. Ambient temperature should be less than during the day, leading to less ECU use at night. However, poor tent insulation and ECU performance can lead to low internal temperatures at night that require heating.

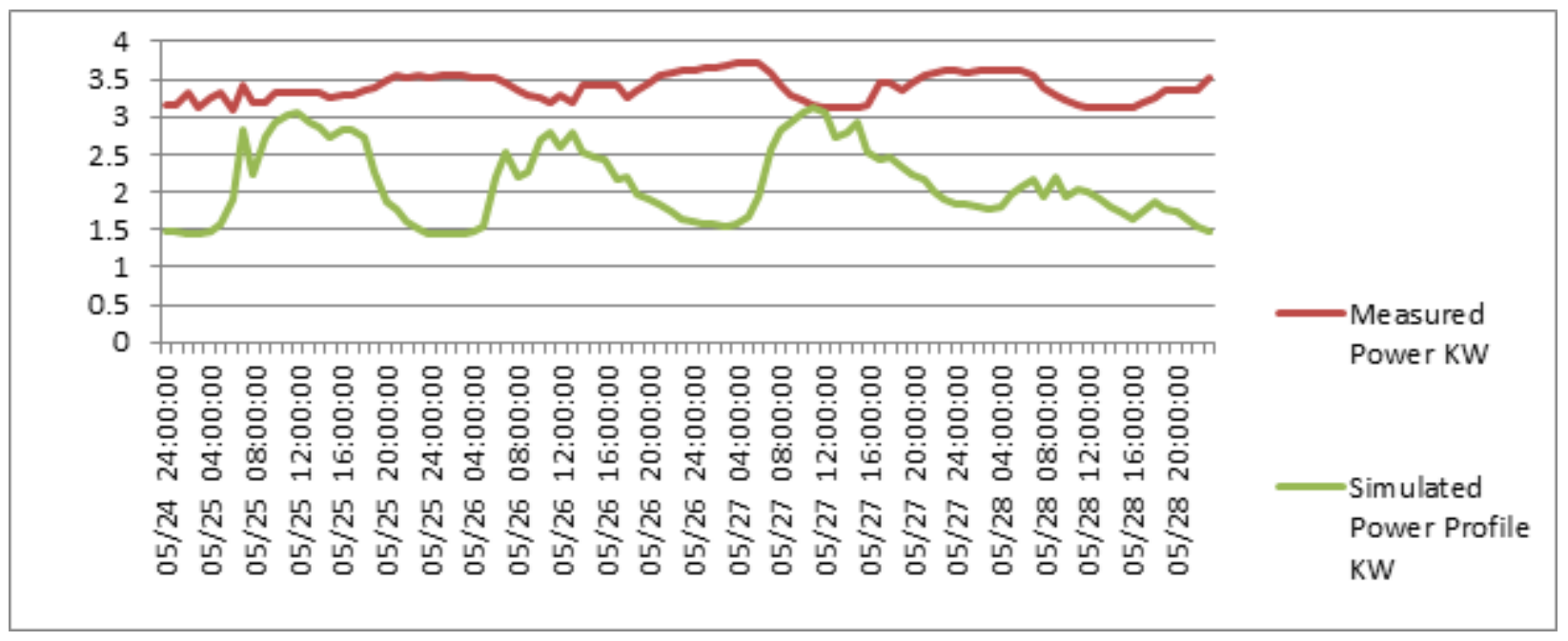

Figure D-3. Average Tent Profile vs Modeled Profile (unoccupied)

Further adjustments to the model reduced the tent fabric thickness from $10 \mathrm{~mm}$ to $6 \mathrm{~mm}$ and increased air infiltration from $90 \mathrm{cfm}$ to $272 \mathrm{cfm}$. The resulting profile is shown in Figure D-4. Variations in the profile are still present, although less pronounced. 


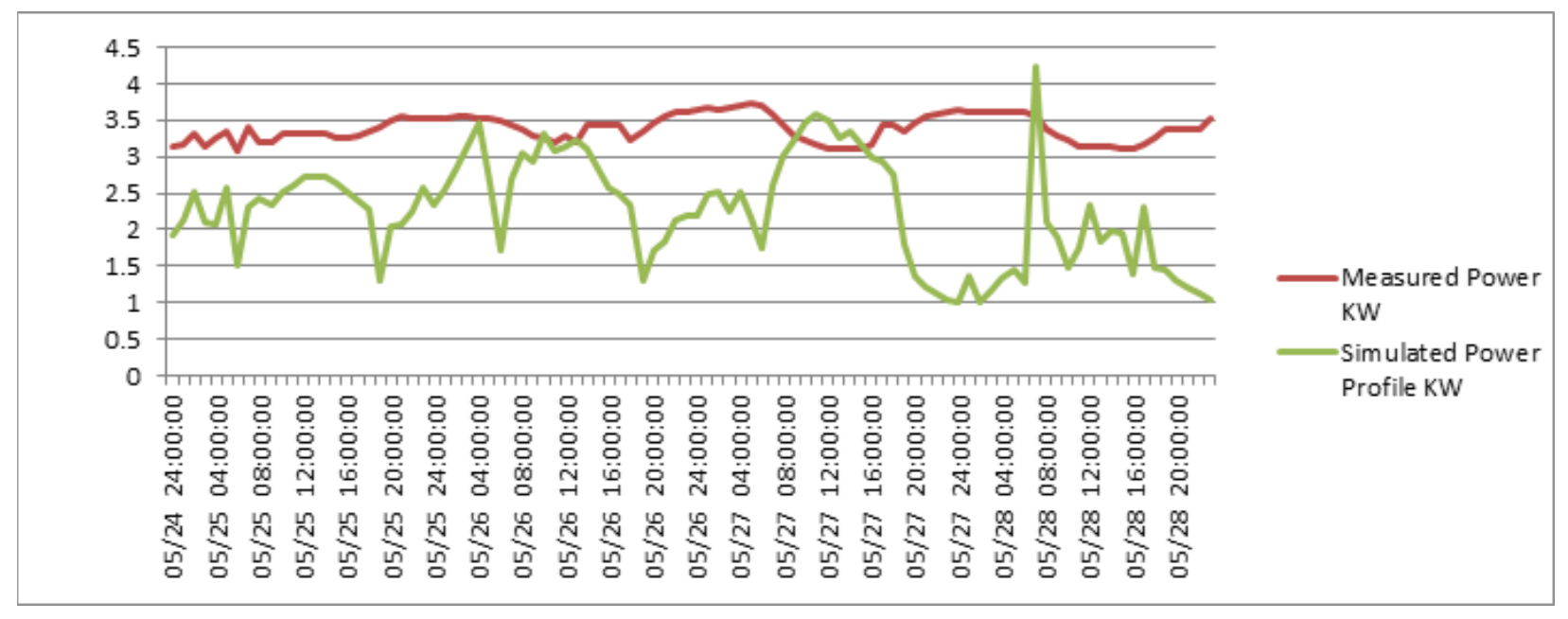

Figure D-4. Average Tent Profile vs Modeled Profile (unoccupied)

After another adjustment of the model, the unoccupied power profiles moved their mean average up (see Figure D-5). The purple line shows outdoor dry-bulb temperature and that the simulated power profile follows this temperature closely.

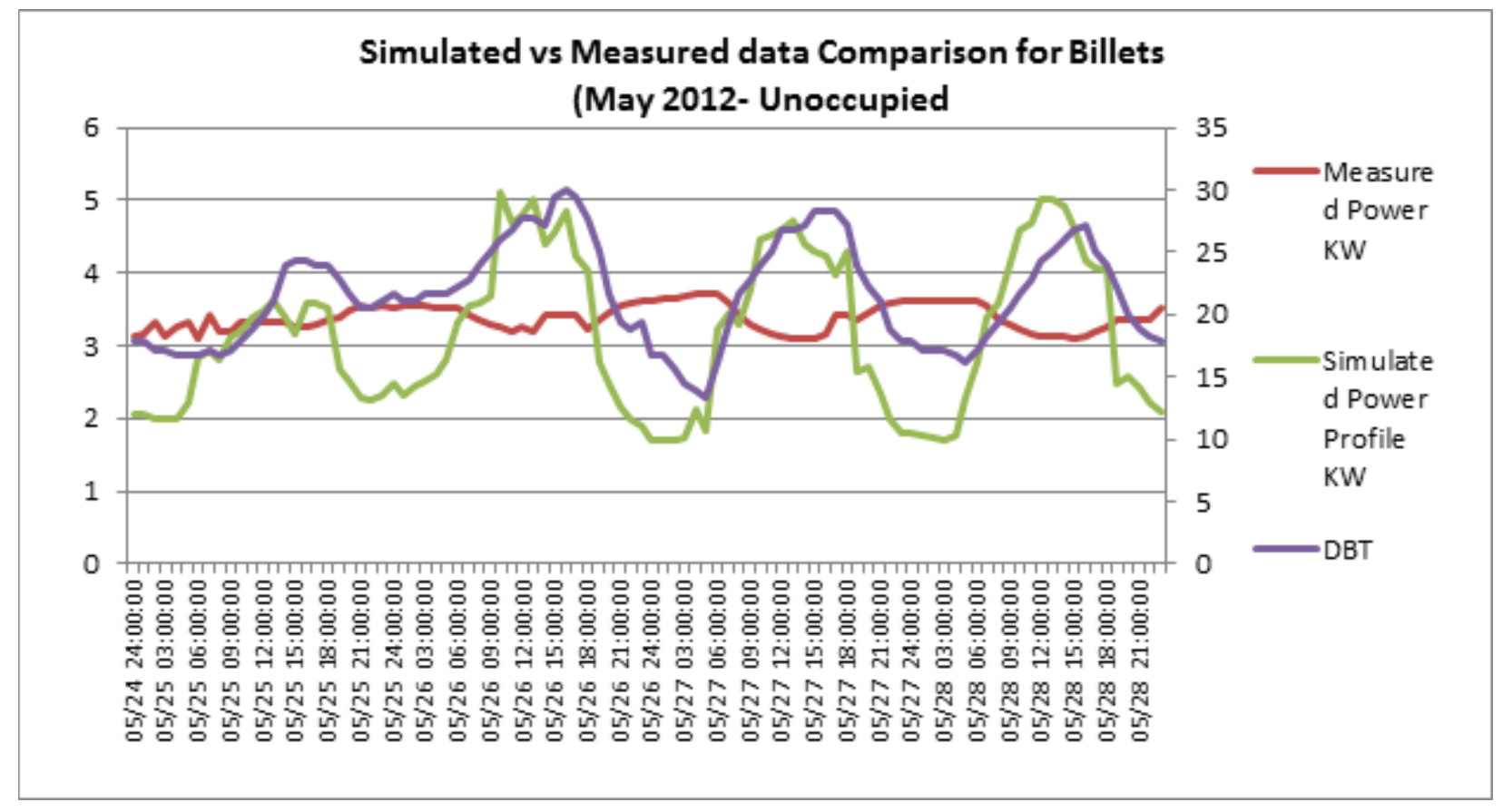

Figure D-5. Average Tent Profile vs Modeled Profile (unoccupied)

The adjustments made for the unoccupied model were applied in a similar manner to the occupied model. Figure D-6 shows the actual field data for an occupied tent versus a modeled tent. The variances are due to the lack of typical plug loads and differences in statistical weather data versus actual weather data. 


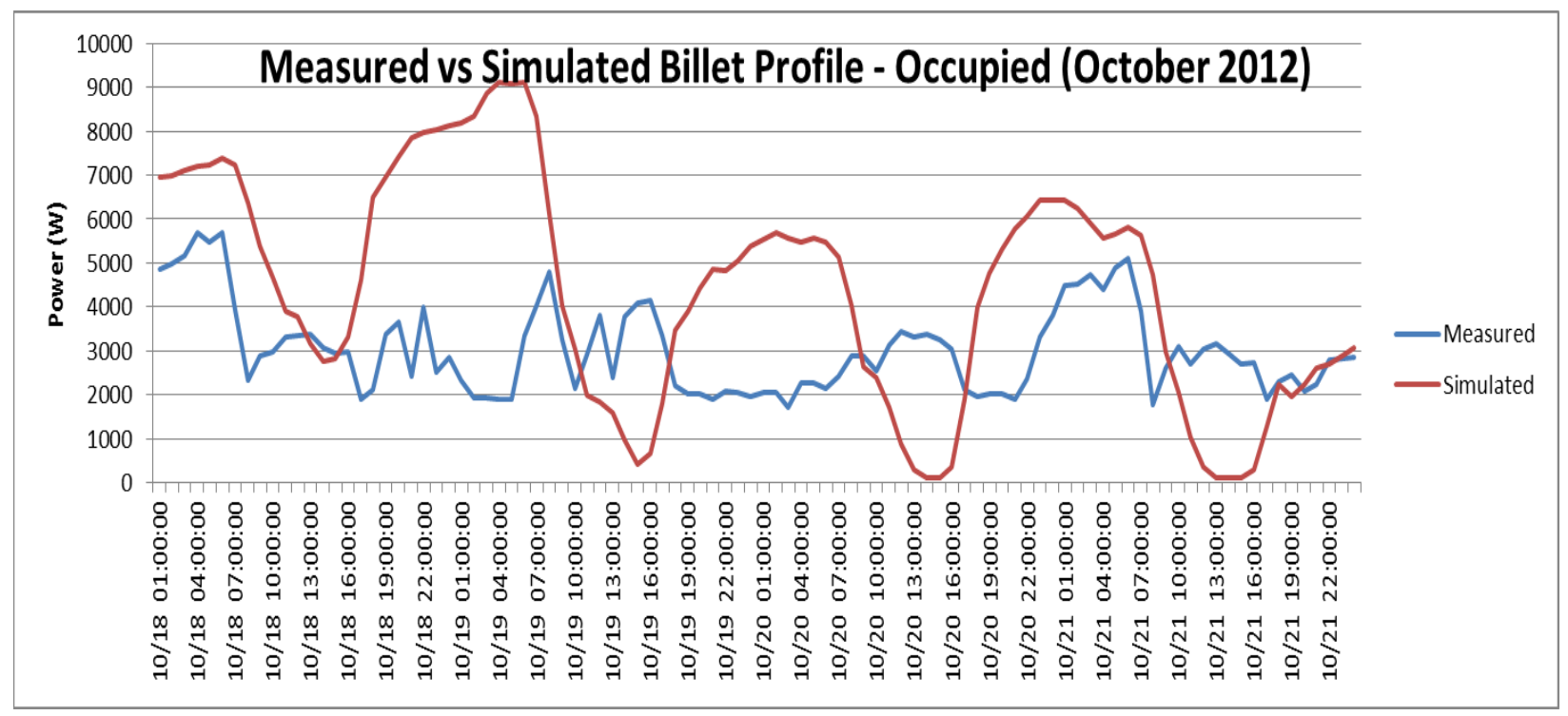

Figure D-6. Tent Real Occupied Power Profile vs Simulated Power Profile

Figure D-7 shows a close tracking of simulated (green) vs actual field data (red) after final adjustments to the occupied model were made. The dark orange trace is the internal tent temperature and the lighter orange shade trace is the outside dry-bulb temperature.

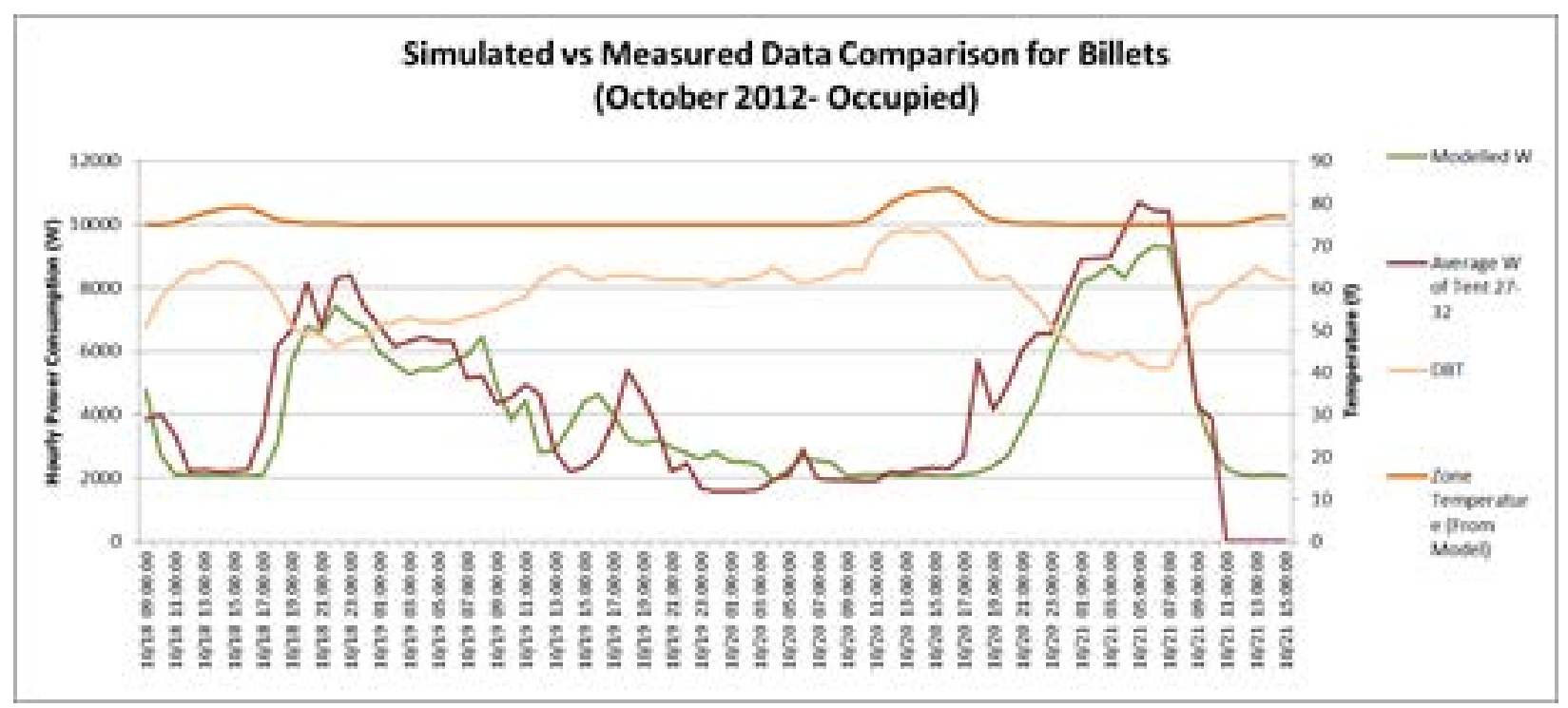

Figure D-7. Tent Real Occupied Power Profile vs Simulated Power Profile

The goal of the calibration was not to create a $100 \%$ match between the data collected during field testing at Fort Devens and simulated data. The Fort Devens field tests were not completely representative of conditions found in actual camps. Plug loads were known to be significantly different. Troop usage patterns were also different, owing to the training nature of the BCIL, when compared to actual field conditions. The calibration efforts allowed a better match in insulation and ECU performance between modeled and field installation. 


\section{Global Annualization and Expansion to All Camp Sizes}

\section{D.1.22 Overview}

After the successful calibration of structures and equipment found in 150-Soldier camps, the same modeling techniques were then applied to structures found in 600-Soldier and 5,000Soldier camps. The baseline 600-Soldier camp was largely composed of the same tent structures found in the smaller 150-Soldier camp. The larger 5,000 Soldier-camp, of a more permanent nature than the smaller installations, used baseline structures composed of more traditional stickframe buildings with insulation and better ECUs. Consequently, replacing baseline structures with SAGE shelters or wood-framed buildings with the best improvement package (highefficiency ECUs and efficient insulation) results in larger electrical energy savings in the 150Soldier and 600-Soldier camps than it does in the 5,000-Soldier camps.

\section{D.1.23 Simulation Data Format}

The simulation data was provided by Pacific Northwest National Laboratory's EnergyPlus modelers in Excel $^{\circledR}$ format. The data was organized by building type - both the baseline and multiple improvement packages - and gives electric power demand hourly for an entire year in fourteen global locations. This also applies to simulated photovoltaic (PV) output data. Water heating data, both baseline (fuel-fired and electrical) and solar-hot water assisted is presented in an annual summary format.

\section{D.1.24 Automated Data Processing (150-Soldier Camp Only)}

The EnergyPlus data for all structures was inserted into a separate Excel file to perform all calculations. Data is arranged in an hourly format for an entire year for fourteen locations. The baseline camp configuration assigns electrical loads to six individual $60 \mathrm{~kW}$ spot generators based on a load schedule provided by the U.S. Army Product Manager, Force Sustainment Systems (PM FSS). Fuel consumption of each spot generator is determined hourly, based upon published fuel-load curves for that generator model.

In cases where the SAGE microgrid is used, a fixed fuel efficiency value is used instead. This value was determined via field testing. Efficiency of the microgrid was found to not vary significantly with a variety of load and climate conditions, during both unoccupied and occupied testing in May/June and October 2012.

\section{D.1.25 Automated Data Processing (600 and 5,000-Soldier Camps)}

The vastly larger amount of EnergyPlus data for the 600 and 5,000-Solider camps made analyzing fuel and energy computation via Excel impractical. The EnergyPlus data was converted from Excel format to comma-separated value (CSV) format and processed by a Scilab/MATLAB ${ }^{\circledR}$ script. The script analyzes the raw data provided by EnergyPlus and reassembles it into location-specific composite load profiles for a number of different technology scenarios. These composite load profiles are saved in separate CSV files. 
A second Scilab/MATLAB script takes each of these composite profiles for each location and calculates hourly fuel consumption for a variable number of spot generators of adjustable size, as well as for a microgrid composed of a variable number of generators of adjustable size. Fuel curves for the generators are adjustable via polynomial equations of $3^{\text {rd }}$ order or higher determined from published generator vendor data.

Finally, the results are saved to an output file in CSV format, structured by location and technology scenario. 



\section{Appendix E \\ Detailed Field Testing Results}

\section{Phase 1 Results}

$\underline{\text { Verification and Testing Phase }}$

This testing phase verified that the microgrid implementation meets the design specifications. The testing used the following scenarios:

- Generator Focused: This scenario focuses on the operation and coordination of the generators.

- PV Focused: This scenario focuses on the power generation capabilities of the PV system.

- Battery Focused: This scenario focuses on the charging and discharging characteristics of the battery system.

- Integration: This scenario focuses on the characteristics of the microgrid as an integrated system.

Evaluation Phase

This testing phase evaluates the performance of the microgrid under various operating modes and loads. The testing used the following scenarios:

- Generators: This scenario examined the behavior of the generators with the PV system and battery system disconnected from the microgrid.

- Battery System Only: This scenario examined the behavior of the battery system with the generators and PV system disconnected from the microgrid.

- Generators and Battery System: This scenario examined the interactions of the generators and battery system with the PV system disconnected from the microgrid.

- Generators, Battery System, PV System: This scenario examined the interactions of the generators, battery system, and PV system.

- Full Operation with Disruption: This scenario examined the behavior of the fully operational microgrid (generators, battery system, and PV system online) with a loss of either generation or load.

\section{E.1.1 Verification and Testing Scenarios}

\section{E.1.1.1 Generator Set Only - Start and Load Ramp}

The battery system and PV system are disconnected from the microgrid, leaving only the generator set and simulated load. This scenario is focused on the performance of the three XQ100 generators as a coordinated group. 
The load bank was configured to ramp up from no load $(0 \mathrm{~kW})$ to the maximum load ( $250 \mathrm{~kW}$ at 0.9 power factor). Testing verified the designed generator set starting sequence. The time elapsed from the start of the ramp to the startup of the each generator was recorded. Subsequently, testing verified that the Hard Wired Load Shed Load Demand (HWLSLD) successfully starts additional generators when the first generator can no longer support the load. The time elapsed from the start of the ramp to the startup of additional generators was recorded. Power quality measurements were performed throughout the test according to the following procedure:

- Connect three XQ100 generator sets, two or three power distribution units (300 kW PDU), load bank transformer and load bank. Energy storage system is not active. Measure power quality (waveform) on any PDU.

- Configure these load steps:

- $55 \mathrm{~kW}$ for $11 \mathrm{~s}$

- $79 \mathrm{~kW}$ for $22 \mathrm{~s}$

- $51 \mathrm{~kW}$ for $200 \mathrm{~s}$

- $72 \mathrm{~kW}$ for $96 \mathrm{~s}$

- $81 \mathrm{~kW}$ for $60 \mathrm{~s}$

- $91 \mathrm{~kW}$ for $60 \mathrm{~s}$

- $101 \mathrm{~kW}$ for $60 \mathrm{~s}$

- $149 \mathrm{~kW}$ for $60 \mathrm{~s}$

- $157 \mathrm{~kW}$ for $60 \mathrm{~s}$

- $167 \mathrm{~kW}$ for $60 \mathrm{~s}$

- $176 \mathrm{~kW}$ for $60 \mathrm{~s}$

- $246 \mathrm{~kW}$ for $8 \mathrm{~s}$

- $240 \mathrm{~kW}$ for $50 \mathrm{~s}$

- Put all three generator sets in AUTO and manually apply a group start signal. All three generators will start and parallel to the load bus. Load bank transformer is energized by the first generator set on the bus. After the Load Demand Enable Delay time is expired (configurable set point $=5 \mathrm{~min}$ ) two of the generator sets will disconnect from the microgrid, cool down, and turn off.

- Load ramp-up can start after there is only one generator set on the bus. The last generator set on the bus will remain on the bus regardless of the load level.

- Set points of interest are: HWLSLD Add Unit Percentage, HWLSLD Add Unit Delay (time), HWLSLD Enable (time). Initial selection is load add $=85 \%$, load drop $=35 \%$ for all three generator sets. 
This test was performed on February 14, 2012. Collected data for this test is shown in Figure E-1. This test is considered to be a success without follow-up needed.

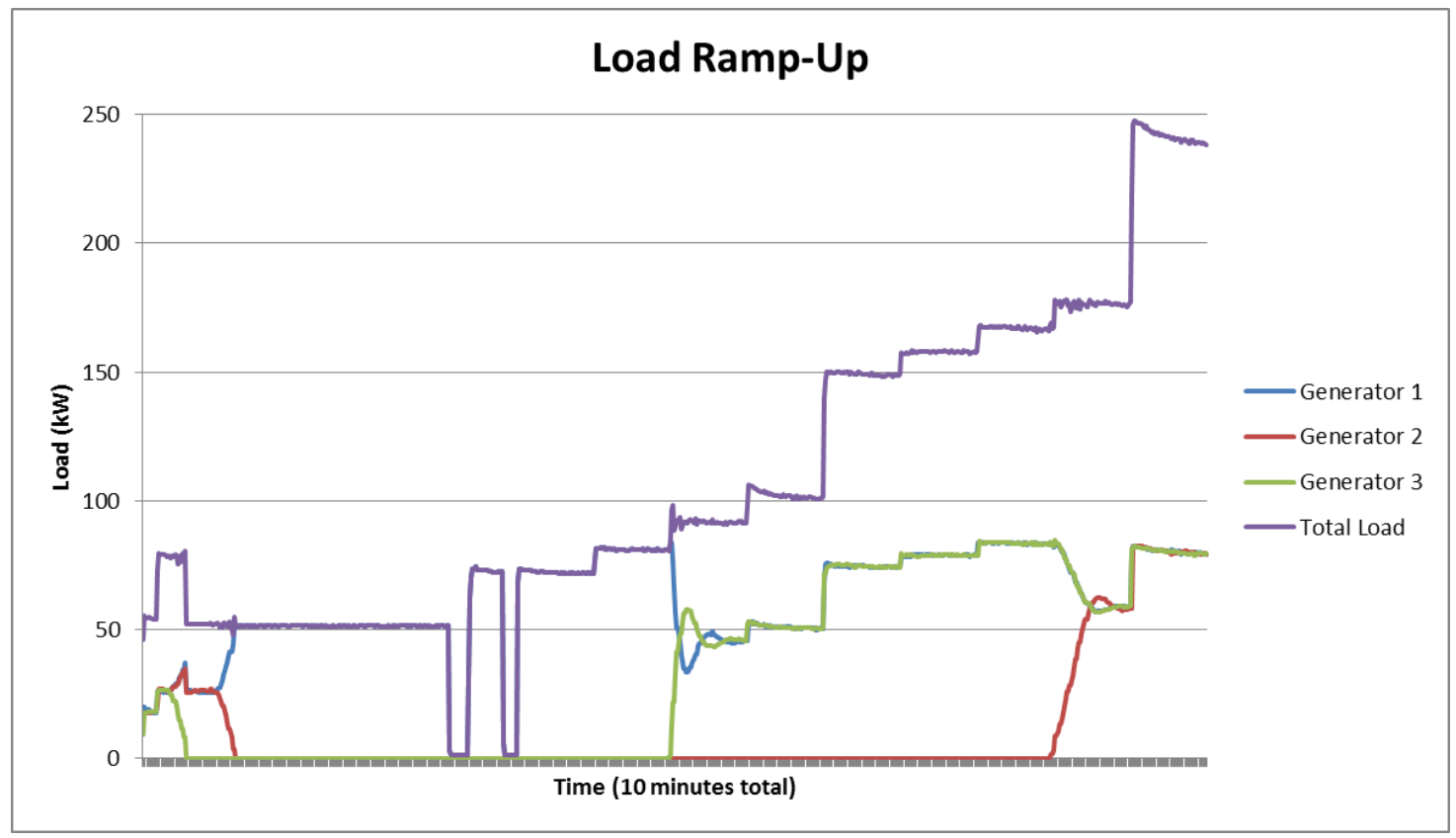

Figure E-1. Ramp-Up Test

\section{E.1.1.2 Load Ramp Down}

Initially, three generators were producing approximately $250 \mathrm{~kW}$ with the load bank set to the corresponding load ( $250 \mathrm{~kW}$ at 0.9 power factor). The load bank was then ramped down from $250 \mathrm{~kW}$ to no load (zero $\mathrm{kW}$ ) over a $90 \mathrm{~s}$ minute period.

Testing verified that the Load Sense Load Demand control successfully disconnects the unnecessary generator sets from the microgrid in response to reduced load demand.

The last generator does not turn off due to manual application of a group start signal. Manual group start is necessary with the inverter secured. The time elapsed from the start of the ramp to the shutdown of the secondary generator was recorded. Subsequently, the time elapsed from the start of the ramp to the shutdown of the primary generator was recorded.

Engineering note: The microgrid will always have a load. Each package generator set has a battery charger and jacket water heater that are always connected. The jacket water heater turns on/off with an internal thermostat. The power transformer in the energy storage container is always connected and there are lights, control power, and HVAC inside the storage container.

Set points of interest were: HWLSLD Drop Unit Percentage, HWLSLD Drop Unit Delay (time), HWLSLD Enable (time), Cool Down Duration (time). Initial selection is load add = $85 \%$, load drop $=35 \%$ for all three generator sets. The following procedure was used in the test:

- From the fully loaded condition above, program the load bank to step to $50 \mathrm{~kW}$. 
- Wait for one generator to shut down.

- Wait for the second generator to shut down.

- Remove the load entirely and verify the last generator does not shut down.

The test was performed on February 14, 2012. This test is considered to be a success without follow-up needed. Collected data for this test is shown in Figure E-2.

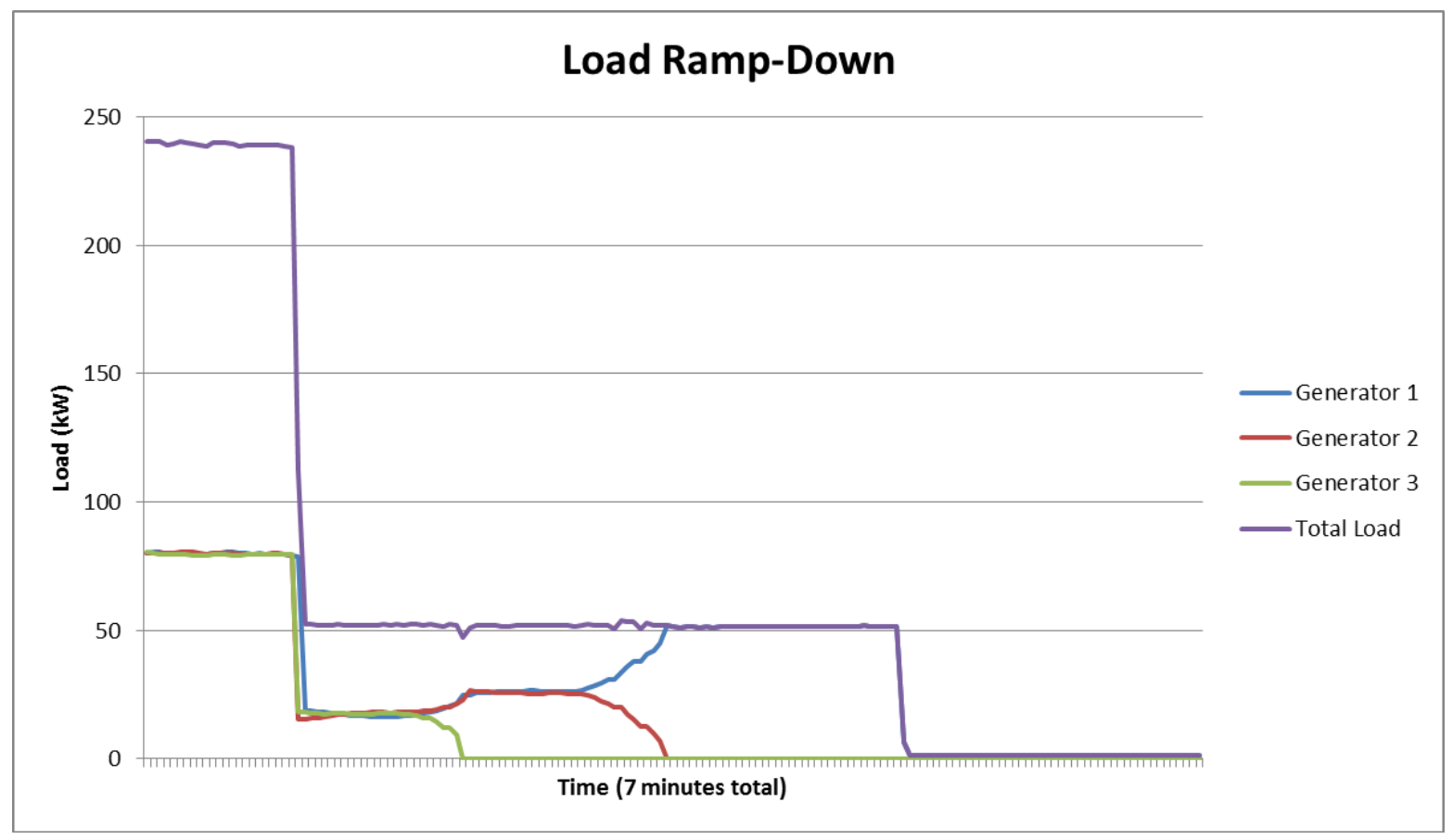

Figure E-2. Load Ramp-Down Test Data

\section{E.1.1.3 Synchronizing Battery System to Generator Set with Low}

The PV system was disconnected from the microgrid. The load bank was set to $100 \mathrm{~kW}$. The generators were started and the test attempted to verify that the battery system synchronizes correctly with the microgrid. Power quality measurements were performed throughout the test.

The end condition of the previous step is microgrid operation in automatic made with load share/load demand enabled, load bank energized, and only parasitic load on the bus. The test was performed according to the following procedure:

- Manually add $100 \mathrm{~kW}$ load. Allow the HWLSLD set points to determine the number of generator sets running.

- Monitor power quality on any power distribution unit.

- Activate the inverter (automatically synchronizes).

The test was performed on February 14, 2012. This test is considered to be a success without follow-up needed. Collected data for this test is shown in Figure E-3. 


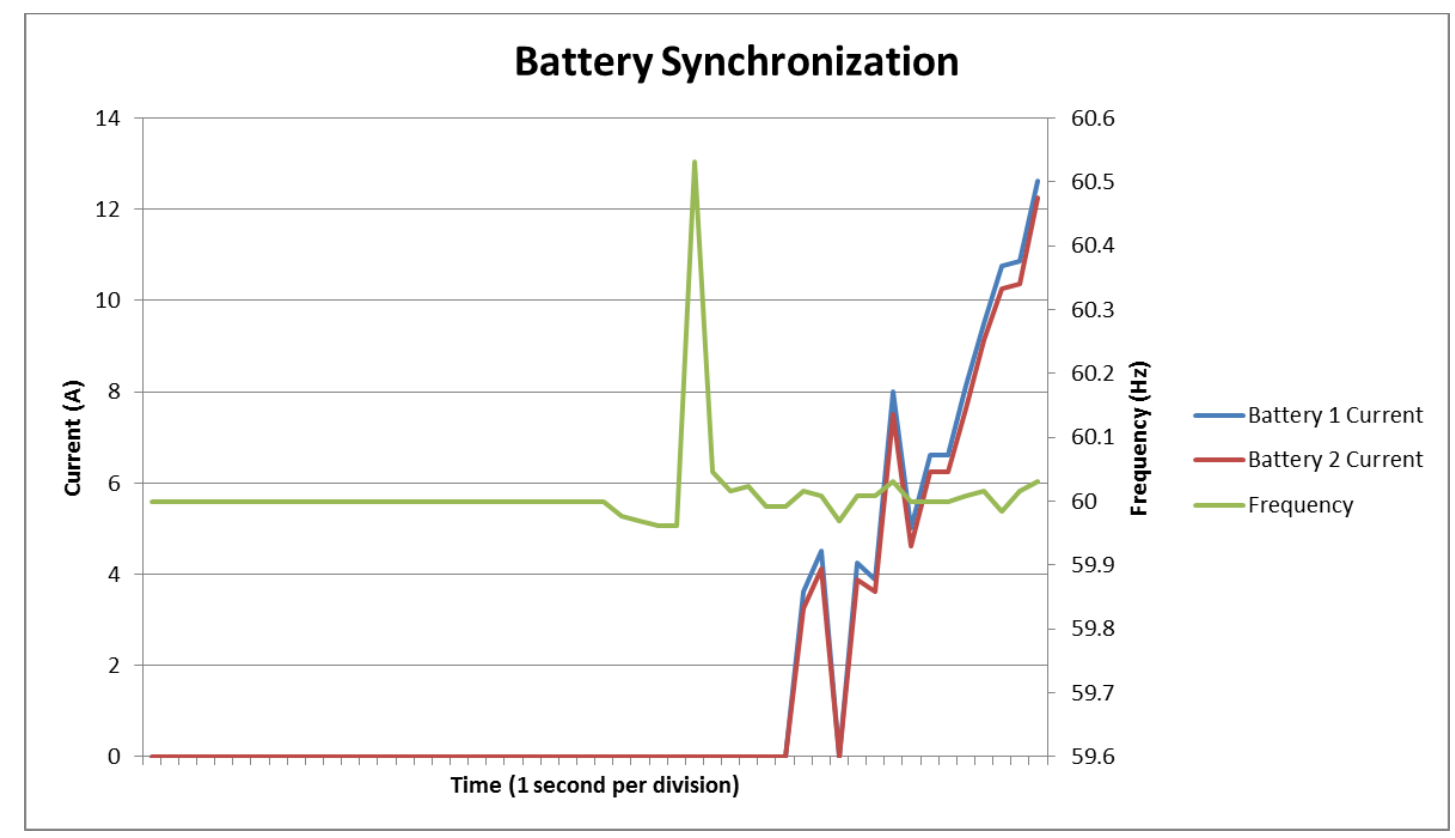

Figure E-3. Battery Synchronization Test Data

\section{E.1.1.4 PV System Synchronization with Generator Set with Low}

This test was to be conducted during daylight hours, preferably midday. The battery system was disconnected from the microgrid. The generators were connected to the microgrid.

The load bank was not used for this test due to low power output of the on-site test PV setup. However, small parasitic loads are present on the microgrid. The generators were started and the PV system was connected to the microgrid. The PV system power output was measured and recorded periodically during the test. The test attempted to verify that the PV system synchronizes correctly to the microgrid. Power quality measurements were performed throughout the test. The test was performed according to the following procedure:

- From the initial conditions above, secure the inverter and remove load from the load bank. Connect the PV system wiring but leave the circuit breaker open. Turn the storage inverter $\mathrm{OFF}$.

- Shut the breaker feeding the PV panel circuit. Monitor power quality on the PDU directly connected to the PV circuit.

- Verify that the PV system/Enphase micro-inverters have locked onto the bus and are feeding power into the microgrid.

- PV power output: 184-284W.

The test was performed on February 16, 2012. This test was considered to be a success without any follow-up needed. 


\section{E.1.1.5 PV Synchronization with Energy Storage System}

This test was conducted during daylight hours. The generators were shut down and disconnected from the microgrid. The load bank was not used for this test due to low power output of the on-site test PV setup. However, small parasitic loads are present on the microgrid. The energy storage system and the PV system were connected to the microgrid. Power quality measurements were performed throughout the test. All of which was an attempt to verify that the PV system synchronizes correctly with the microgrid when only the energy storage system is online. The test was performed according to the following procedure:

- Set the load bank to zero load.

- Verify the energy storage system is connected and synchronized to the microgrid.

- Verify the generators are off.

- Shut the PV system connection breaker and verify it synchronizes to the grid and puts out power. Power output: 184-284W.

The test was performed on February 16, 2012 and was considered successful, no follow-up needed.

\section{E.1.1.6 PV System Synchronization with Energy Storage System and Generators}

This test was conducted during daylight hours. The running generators and the energy storage system were connected to the microgrid. The load bank was not used for this test due to low power output of the on-site test PV setup. However, small parasitic loads are present on the microgrid. The PV system was connected to the microgrid, which measured and recorded the system's output. The test was to verify that the PV system synchronizes correctly to the microgrid. Power quality measurements were performed throughout the test. The test was performed according to the following procedure:

- Set the load bank to zero load.

- Verify at least one generator is running and connected to the microgrid.

- Verify the energy storage system is connected and synchronized to the microgrid.

- Shut the PV system connection breaker and verify it synchronizes to the grid and puts out power. Power output: 184-284W.

The test was performed on February 16, 2012 and was considered successful, no follow-up needed.

\section{E.1.1.7 Partial Loss of Load - Full Operation with Disruption}

The generators and energy storage system were connected to the microgrid. The battery system was initially fully charged. The load bank was set to $166 \mathrm{~kW}$. 
One to two minutes after test start, the load was reduced as quickly and safely as possible by $50 \mathrm{~kW}$. Power quality measurements were performed throughout the test. This test was to determine the behavior of the generators and energy storage system during a serious system disruption. After a first run of the test, the energy storage system was disconnected from the microgrid to directly measure the effect of the energy storage system on microgrid stability. The test was performed according to the following procedure:

- Verify generators are running and connected to the microgrid.

- Verify load bank is set to $166 \mathrm{~kW}$.

- Verify energy storage system is on and connected to the microgrid.

- Monitor power quality on any PDU.

- Set the load bank set point to $80 \mathrm{~kW}$ and monitor grid frequency closely.

The test was performed on February 14, 2012, it was considered successful, no follow-up needed.

The results from this test are shown in Figure E-4.

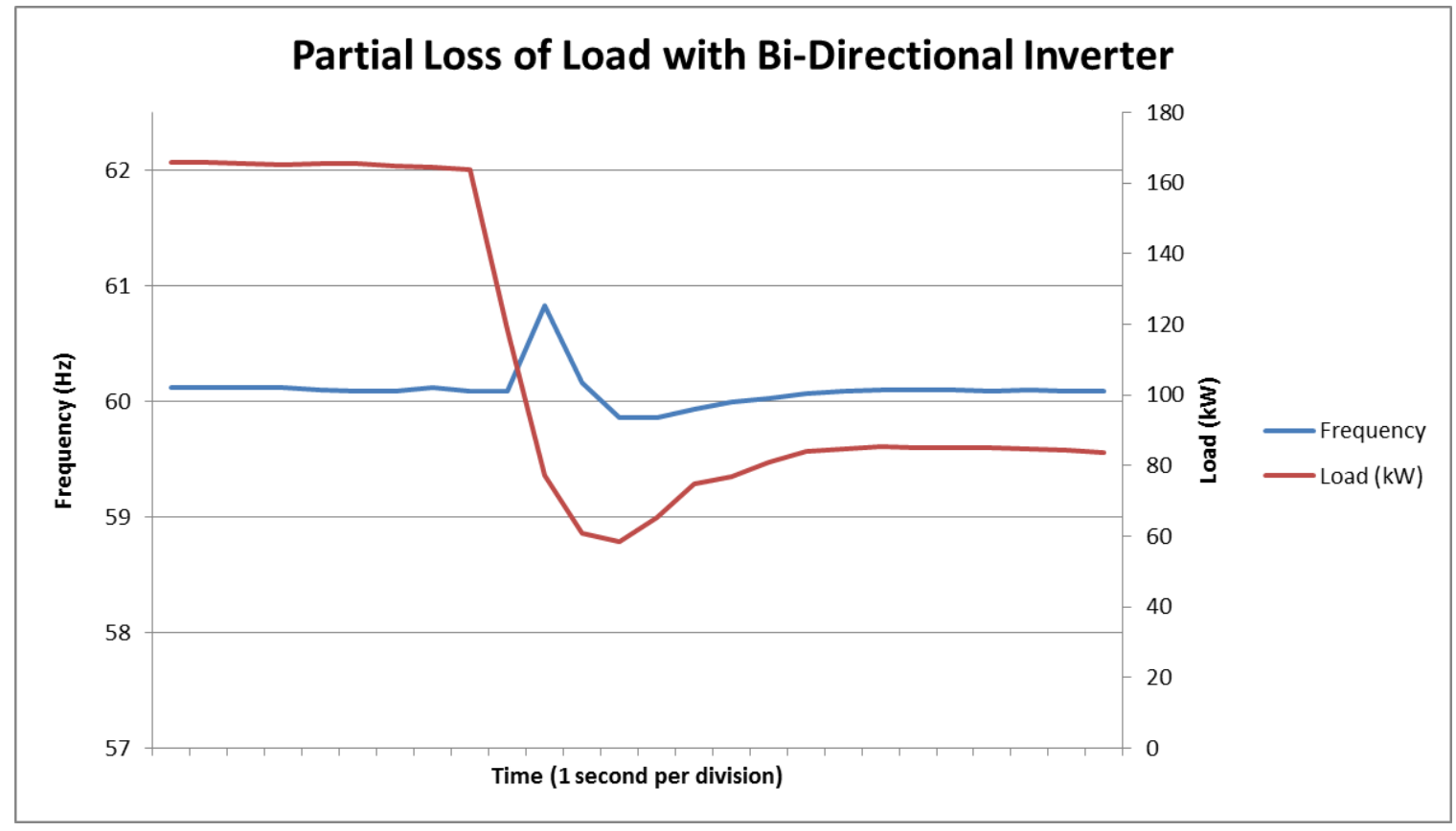

Figure E-4. Partial Loss of Load with Bidirectional Inverter

\section{E.1.1.8 Total Loss of Load - Full Operation with Disruption}

In this scenario, the generators and energy storage system were connected to the microgrid. The battery system was initially fully charged. The load bank was set to $166 \mathrm{~kW}$.

One to two minutes after test start, the load was dropped to $0 \mathrm{~kW}$ as quickly and safely as possible. Power quality measurements were performed throughout the test. This test attempted 
to determine the behavior of the generators and energy storage system. After a first run of the test, the energy storage system was disconnected from the microgrid to directly measure the effect of the energy storage system on microgrid stability. At the completion of testing, the generators were allowed to completely charge the battery system. The test was performed according to the following procedure:

- Verify generators are running and connected to the microgrid.

- Verify load bank is set to $155 \mathrm{~kW}$.

- Verify energy storage system is on and connected to the microgrid.

- Monitor power quality on any PDU.

- Set the load bank set point to $0 \mathrm{~kW}$, then immediately back to $155 \mathrm{~kW}$ and monitor grid frequency closely.

- Repeat the test without the energy storage system connected to the microgrid.

The test was performed on February 14, 2012, it was considered successful, no follow-up needed. The results are shown in Figure E-5 and Figure E-6.

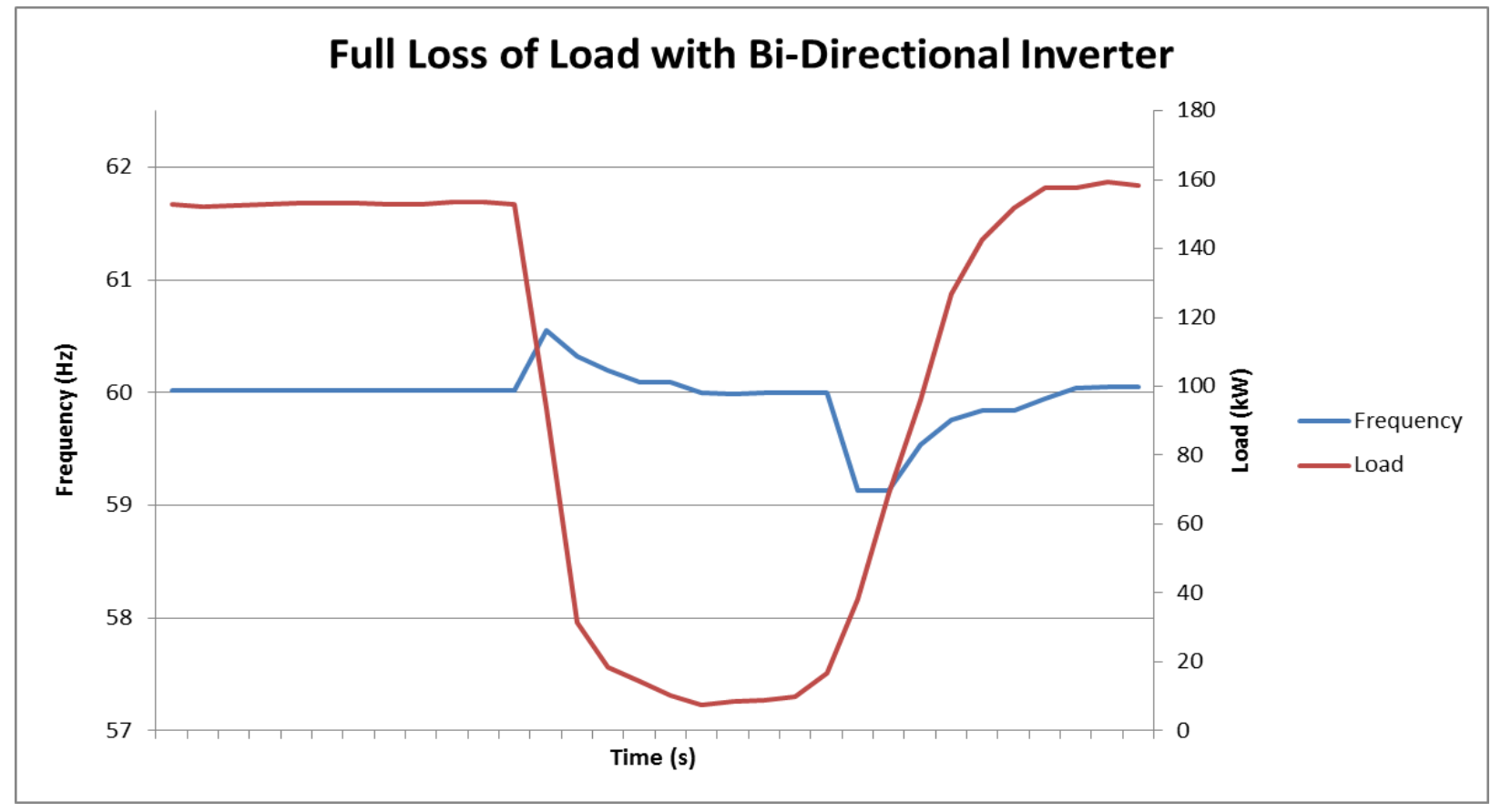

Figure E-5. Full Loss of Load with Bidirectional Inverter 


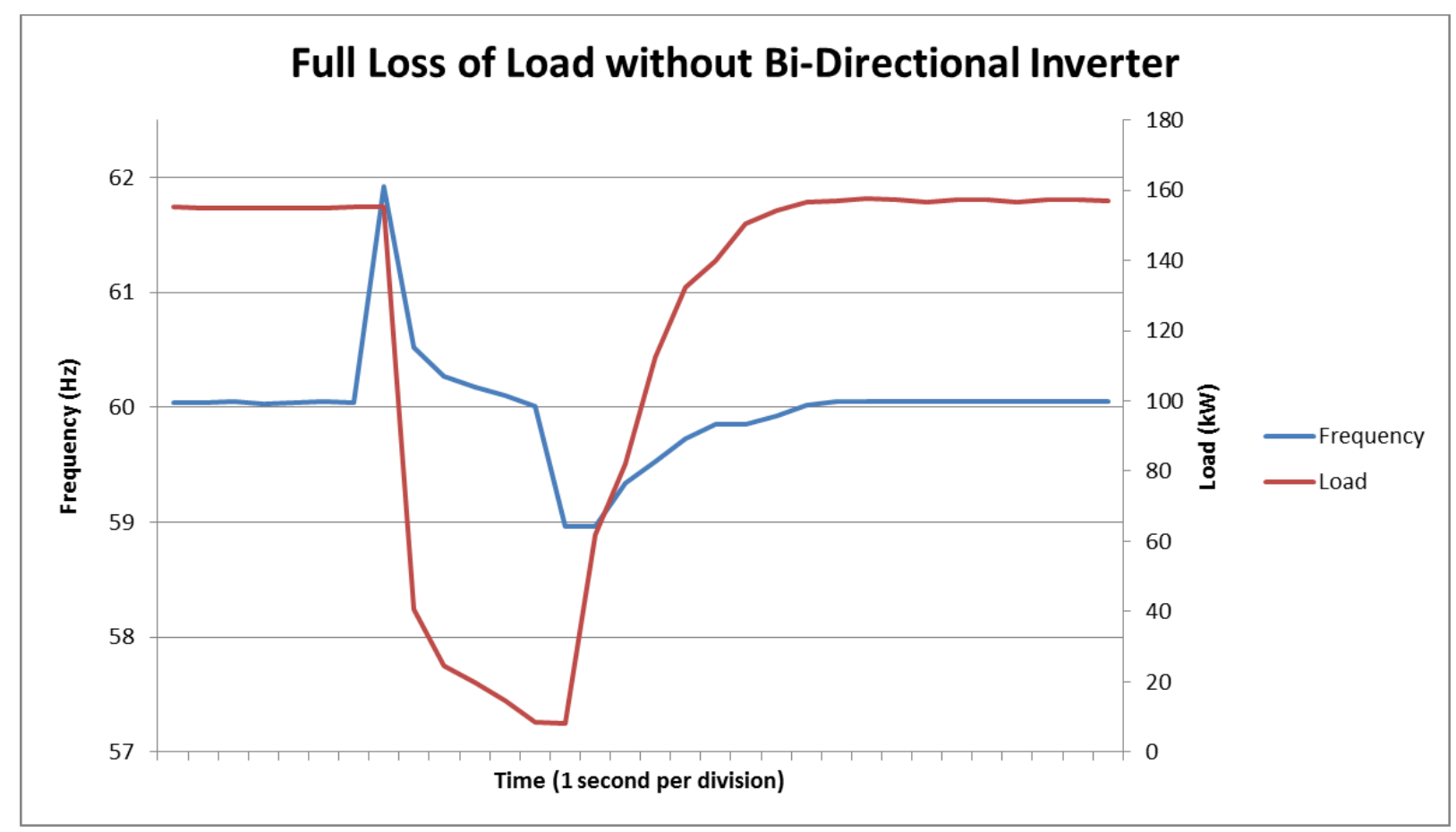

Figure E-6. Full Loss of Load without Bidirectional Inverter

\section{E.1.1.9 Loss of Generation - Full Operation with Disruption}

The generators and energy storage system were connected to the microgrid. Initially, the battery system was fully charged. The load bank was set to $160 \mathrm{~kW}$. The initial measurements were to verify that the battery system was fully charged within the specified tolerance. After a steady-state load sharing condition was reached, one generator set was shut down. After a short wait - to achieve a steady state plant - the second generator set was shut down. These steps were repeated until all three generators were off and the plant was running on the inverter only. Power quality measurements were performed throughout the test. At the completion of testing, the generators were allowed to completely charge the battery system. The test was performed according to the following procedure:

- Verify generators are running and connected to the microgrid.

- Verify load bank is set to $155 \mathrm{~kW}$.

- Verify energy storage system is on and connected to the microgrid.

- Monitor power quality on any PDU.

- The system will stabilize with three generator sets running and equally sharing load. Reduce the load to $150 \mathrm{~kW}$. Wait for steady-state operation.

- Manually turn off generator \#3 (Emergency Stop). 
- Expected operation is for the remaining two generator sets to equally share the total load. The energy storage system will momentarily pick up load in the transient. Wait for steadystate operation.

- Manually turn off generator \#2 (Emergency Stop). The inverter and one generator set will supply the excess of one generator capacity.

- Turn off generator \#1 (Emergency Stop). Run with the inverter only for $30 \mathrm{~s}$.

- Restart all three generator sets in automatic control and allow the battery bank to charge.

The test was performed on February 14, 2012, it was not considered successful because the energy storage system was unable to regulate a stable $60 \mathrm{~Hz}(59.1-60.9 \mathrm{~Hz})$ grid frequency. A follow-up test was conducted by Caterpillar at a later date in March 2012 which was successful. The results are shown in Figure E-7, Figure E-8, and Figure E-9.

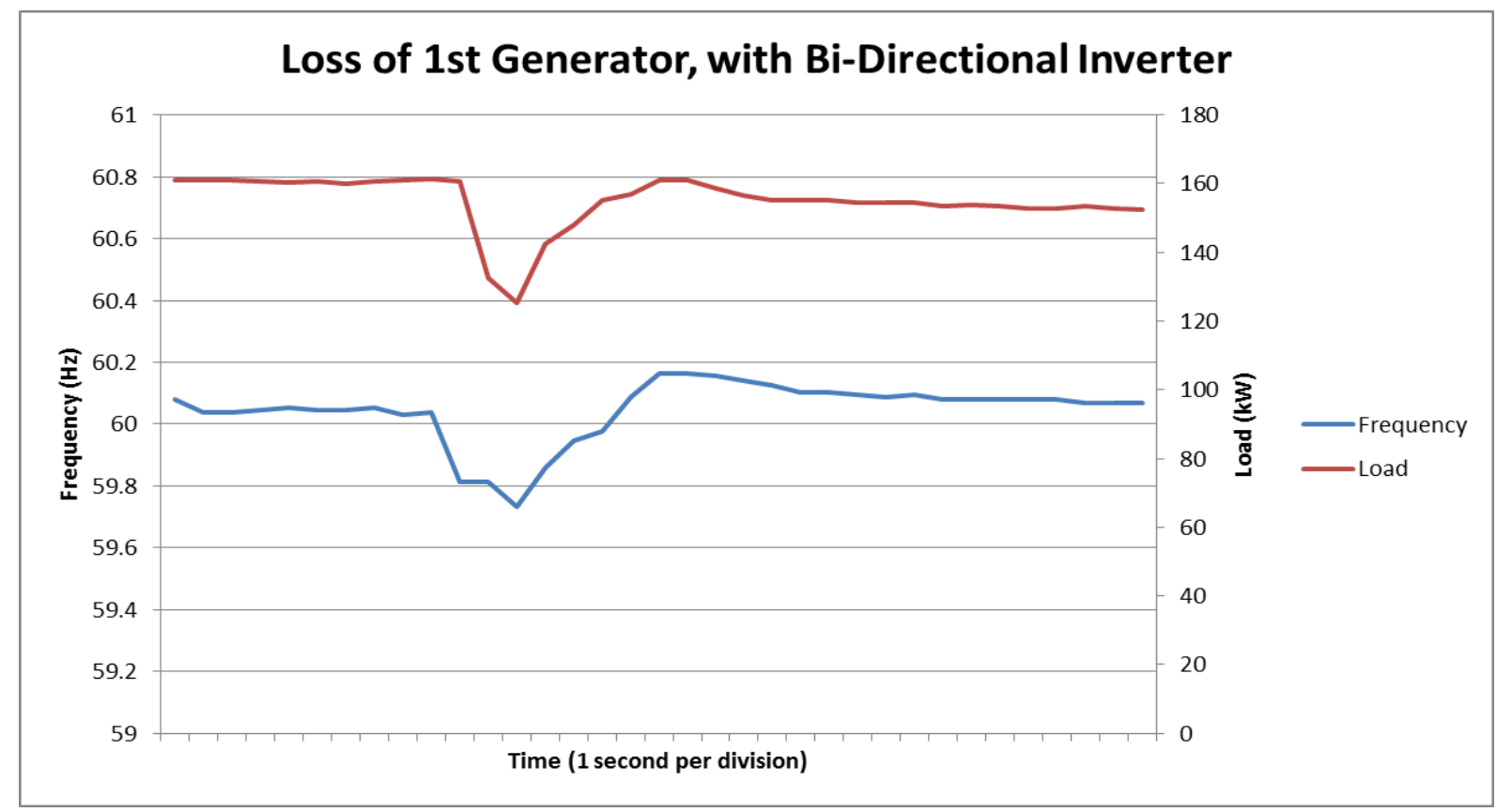

Figure E-7. Loss of First Generator 


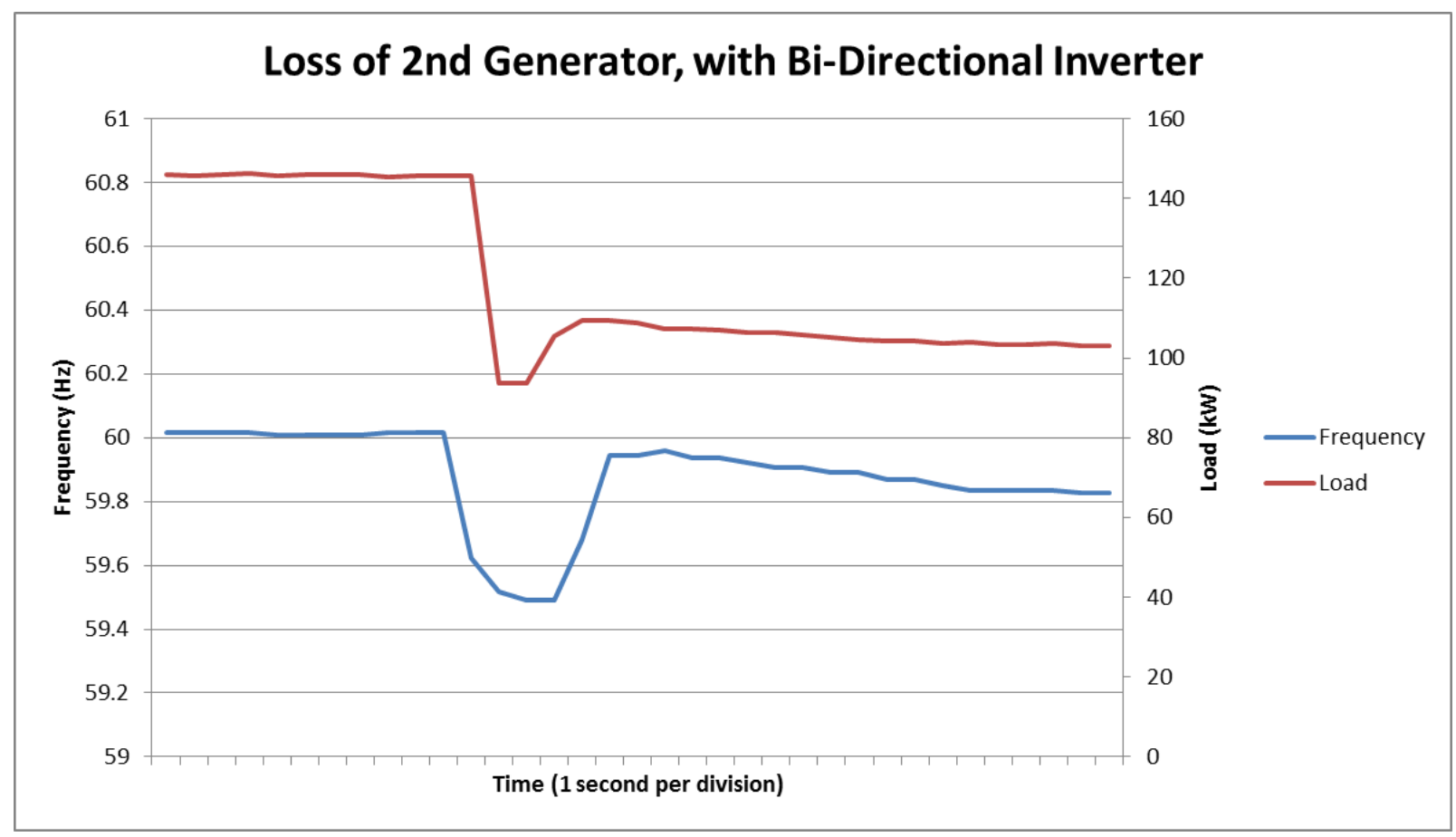

Figure E-8. Loss of Second Generator

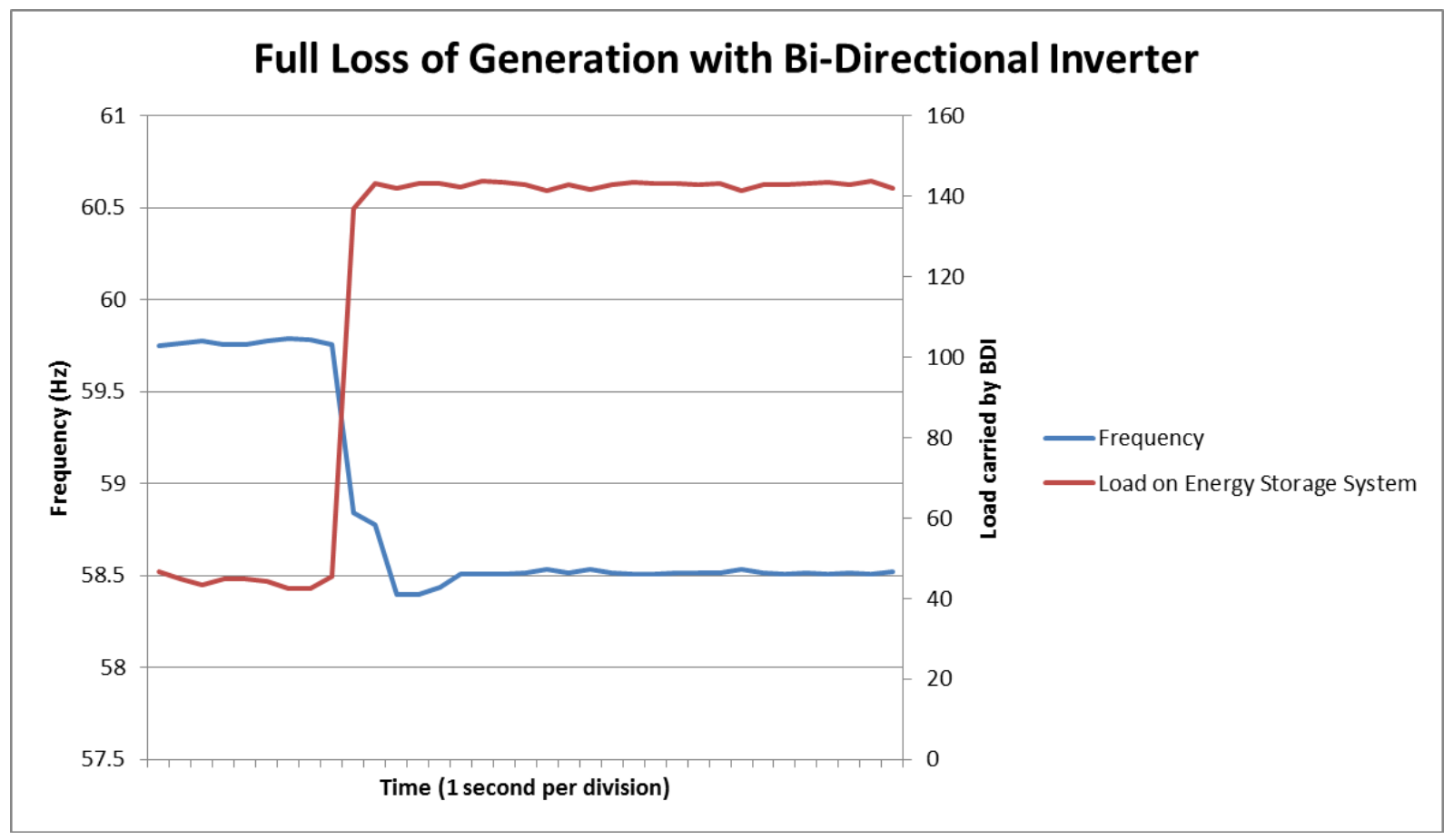

Figure E-9. Loss of all Generators 


\section{E.1.1.10 Overall Daily Base Camp Load Profile with Cycling Inductive Load (Generators and Energy Storage only)}

In this scenario, the PV system was disconnected from the microgrid. The generators and battery system were connected to the microgrid. Cycling inductive load was simulated using the load bank. Air conditioner load was simulated as a quantity of eight $5 \mathrm{~kW}$ loads (total $40 \mathrm{~kW}$ ). A block load of $50 \mathrm{~kW}$ at 0.5 power factor was added to a simulated camp "base load" to demonstrate the starting eight air conditioner units simultaneously.

Initially, measurements were to verify that the battery system was fully charged within the specified tolerance. The test was started, and after 1 minute the load step was applied. After another minute, the load step was removed. This cycle was repeated once. Power quality measurements were performed throughout the test. At the completion of testing, the generators were allowed to completely charge the battery system. The test was performed according to the following procedure:

- Monitor power quality on any PDU. From the running plant conditions above, after the battery bank is charged, apply Load 1 using the load bank.

- $\operatorname{Load} 1$ = $100 \mathrm{~kW}, 0.9$ power factor, $111 \mathrm{kVA}, 48 \mathrm{kVAR}$

- Wait for steady-state conditions with two generator sets sharing the load.

- Change the load bank set point to Load 2 to apply the load in one step.

- $\operatorname{Load} 2=150 \mathrm{~kW}, 0.74$ power factor, $202 \mathrm{kVA}, 135 \mathrm{kVAR}$

- Wait 5 minutes. Apply Load 1. Wait 5 minutes. Apply Load 2.

The test was performed on February 14, 2012, it was considered successful, no follow-up needed. The results are shown in Figure E-10. 


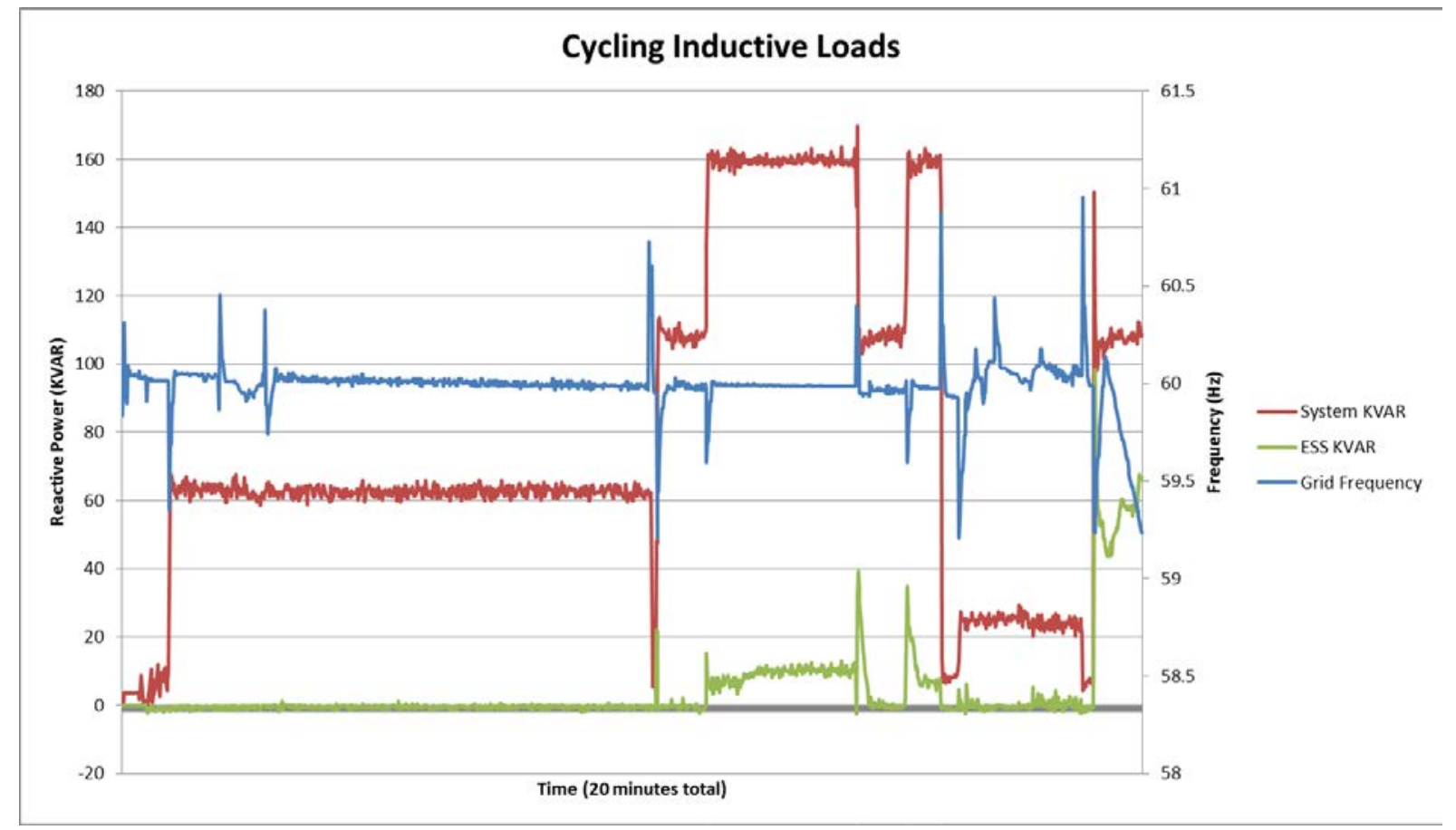

Figure E-10. Cycling Inductive Loads

\section{E.1.1.11 Overall Daily Base Camp Load Profile with Cycling Inductive Load - Energy Storage System ONLY}

This test is a repeat of test C.1.10 without the generators running. The test was performed according to the following procedure:

- Verify the energy storage system is charged (>80\% state of charge).

- Apply Load 1. Turn off all generator sets. Wait 5 min.

- Apply Load 2. Wait 1 min.

- Apply Load 1. Wait 1 min.

- Apply Load 2. Wait 1 min.

- Group-start all the generator sets.

- Set the load bank set point to zero and allow the batteries to fully charge.

The results from this test are shown in Figure E-11. The test was performed on February 14, 2012.

This initial test is not considered to be a success because the energy storage system is unable to regulate a stable $60 \mathrm{~Hz}(59.1-60.9 \mathrm{~Hz})$ grid frequency. A follow-up test was conducted by Caterpillar at a later date in March 2012 which was successful. 


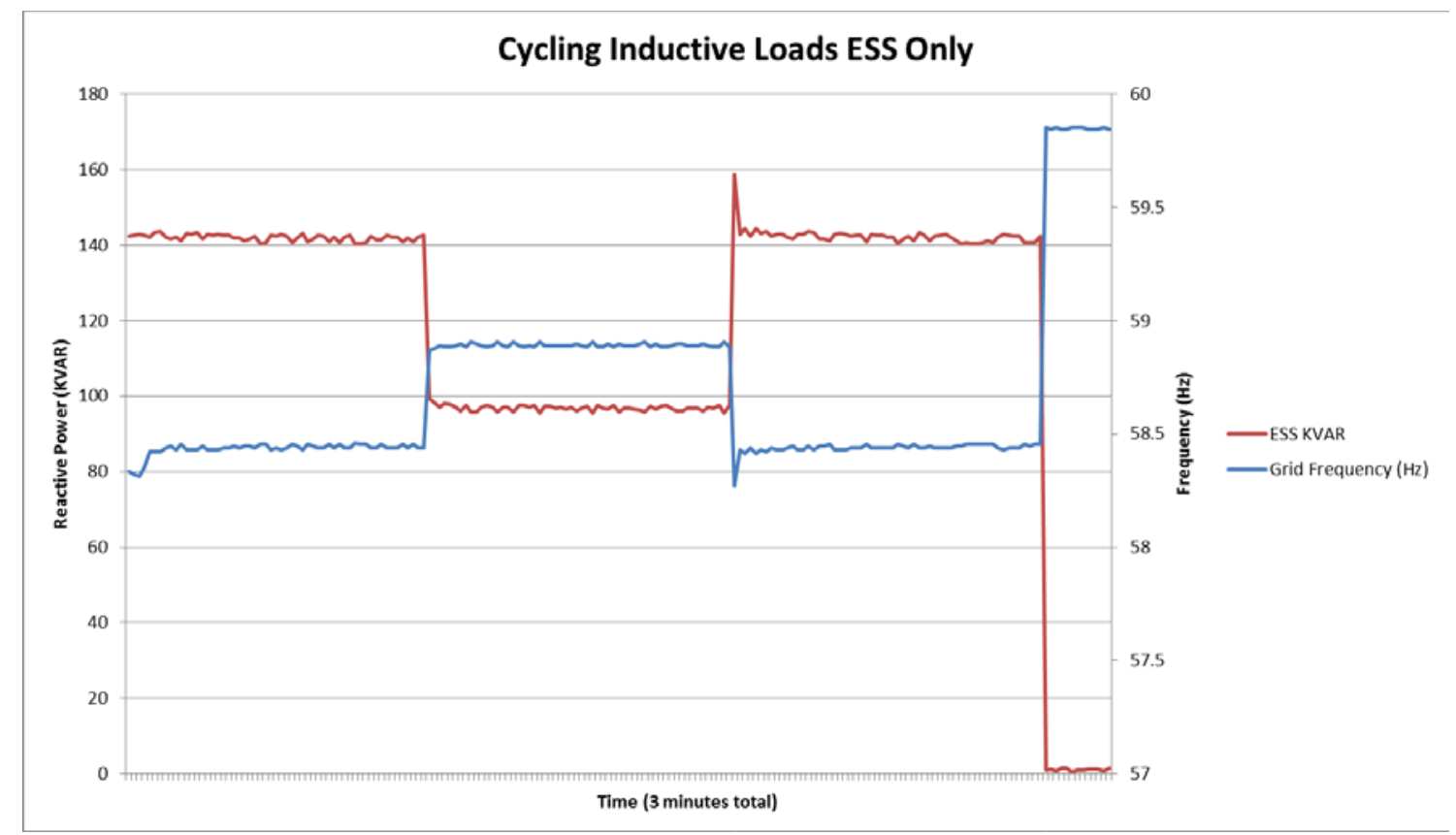

Figure E-11. Cycling Inductive Loads, Energy Storage System Only

\section{E.1.1.12 Battery System Discharging}

With the generators shut down, the load bank was set at $100 \mathrm{~kW}$. The battery system charge state was measured and recorded as a function of time. The discharge time and rate were to indicate the specified capacity of the battery system and discharge rate within specified tolerances. The test was performed according to the following procedure:

- From the test above with the generator set(s) running and a fully charged battery $(97 \%$ state of charge [SOC]), the load bank reached $100 \mathrm{~kW}$.

- Manually secure all running generator sets and leave the applied load carried by the inverter.

- Discharge to a state of charge that will shut the inverter down. Measure the time to fully discharge the battery bank. This is also the time available for "silent camp" operation at $100 \mathrm{~kW}$ customer load.

- Time to discharge from 97\% to inverter shutdown: 1 hour 45 minutes.

- SOC1: $34 \%$, SOC2: $22 \%$

The test was performed on February 14, 2012. The results are shown in Figure E-12.

This initial test is considered to be a success; however, the divergence of the SOC curves was of some concern. Follow-up was needed to determine a suggested course of action to plan for these divergences in the future. A follow-up test was conducted by Caterpillar at a later date in March 2012 and was successful. 


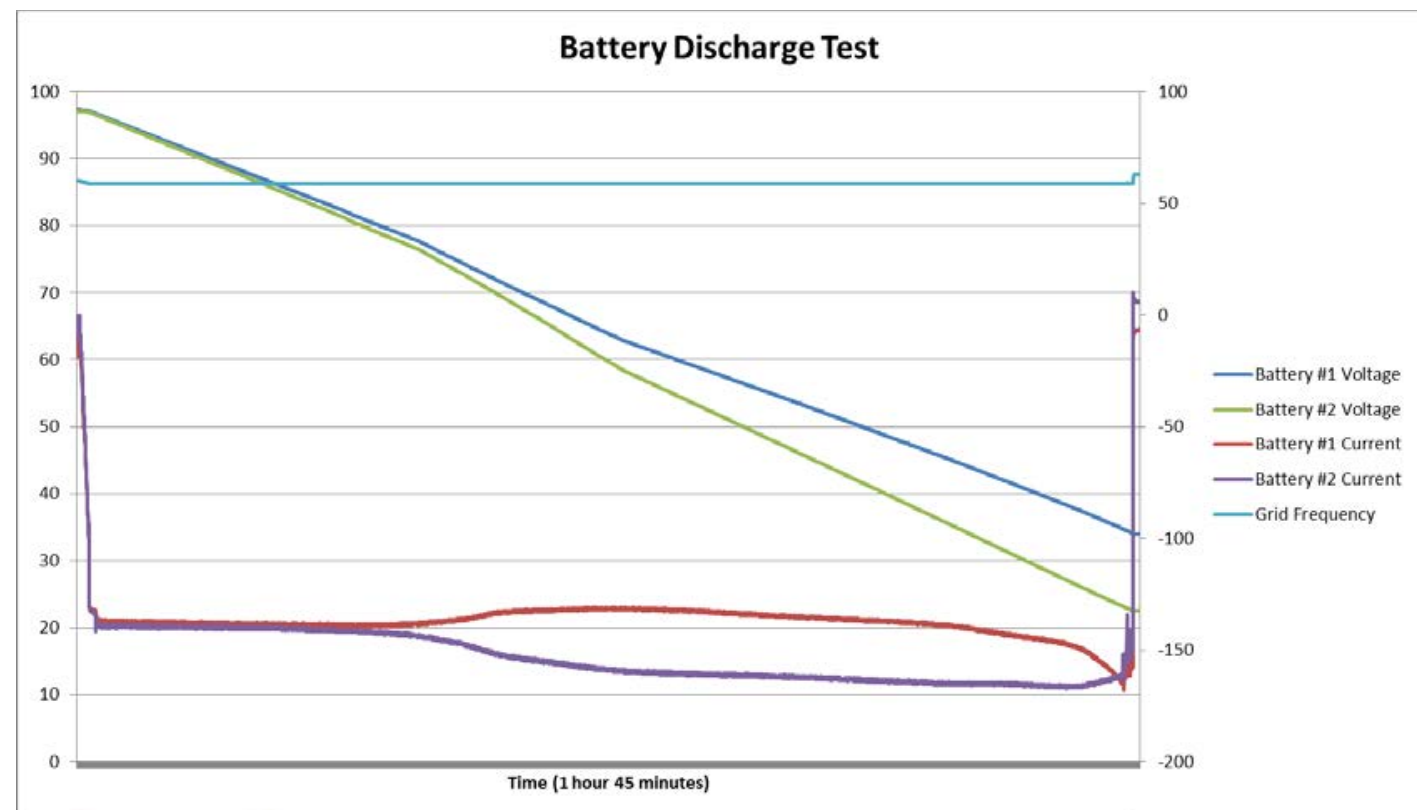

Figure E-12. Battery Discharge Test

\section{E.1.1.13 Battery System Charging}

The load on the microgrid was to be disconnected or set to zero. The generator set group was started up. The battery system charge state was measured and recorded as a function of time. The time required for the battery system to fully charge was also measured and recorded. The charging time and rate were to comply with the specified values within the specified tolerances. The test was performed according to the following procedure:

- From the fully discharged state above, set the load bank set point to zero.

- Group-start the generators. Allow the system to charge the batteries. Allow the HWLSLD set points to determine the number of generator sets running.

- At $100 \%$ SOC, record the charge time.

- Engineering notes: The initial charge will raise the battery voltage until a charge current of 108 amps. Charging continues at this steady current. Voltage will continue to be raised until a limit of $435 \mathrm{VDC}$ is reached. At this point, the charge current will taper as the voltage is held constant. The total charge time from zero to $100 \%$ state of charge is expected to be 8 to 12 hours.

- System charging started at 3:15 p.m. on February 14, 2012.

- Batteries were verified at 92.8\% SOC1 and 90.6\% SOC2 at 12:00 a.m. on February 15, 2012. The generators were not running at that time. It is unknown when the generators shut off. Batteries were not fully charged. Test data from 3:00 p.m. until 11:59 p.m. February 14, 2012, is missing. 
The test was performed on February 14, 2012. This initial test was not considered to be a success. Follow-up on missing data and generator shut-down time was requested. A follow-up test was conducted by Caterpillar at a later date in March 2012 and was successful.

\section{E.1.1.14 Fuel Consumption - Generators Only}

The generators provided the only power to the load. The load bank was configured to simulate the overall daily base camp load profile, which includes the kitchen. The two 12-hour test segments listed in Figure E-13 were combined into one 24-hour load profile. During the testing, the consumption of diesel fuel was measured and recorded. The test was performed according to the following procedure:

- Verify all data logging systems are connected and functional.

- Configure the load bank program to automatically conduct the 24 load steps in Figure E-13, with a one-hour interval between steps.

- Group-start the generator sets and apply the first load step.

- The load share/load demand set points will determine the correct number of generator sets to run for each load.

- Total fuel consumption via calibrated fuel meter was 1227.3 liters. This meter has a calibration traceable to the National Institute of Standards and Technology (NIST). Total consumption via generator internal sensors was 1309 liters. This means that the internal sensors are not considered entirely accurate, as expected.

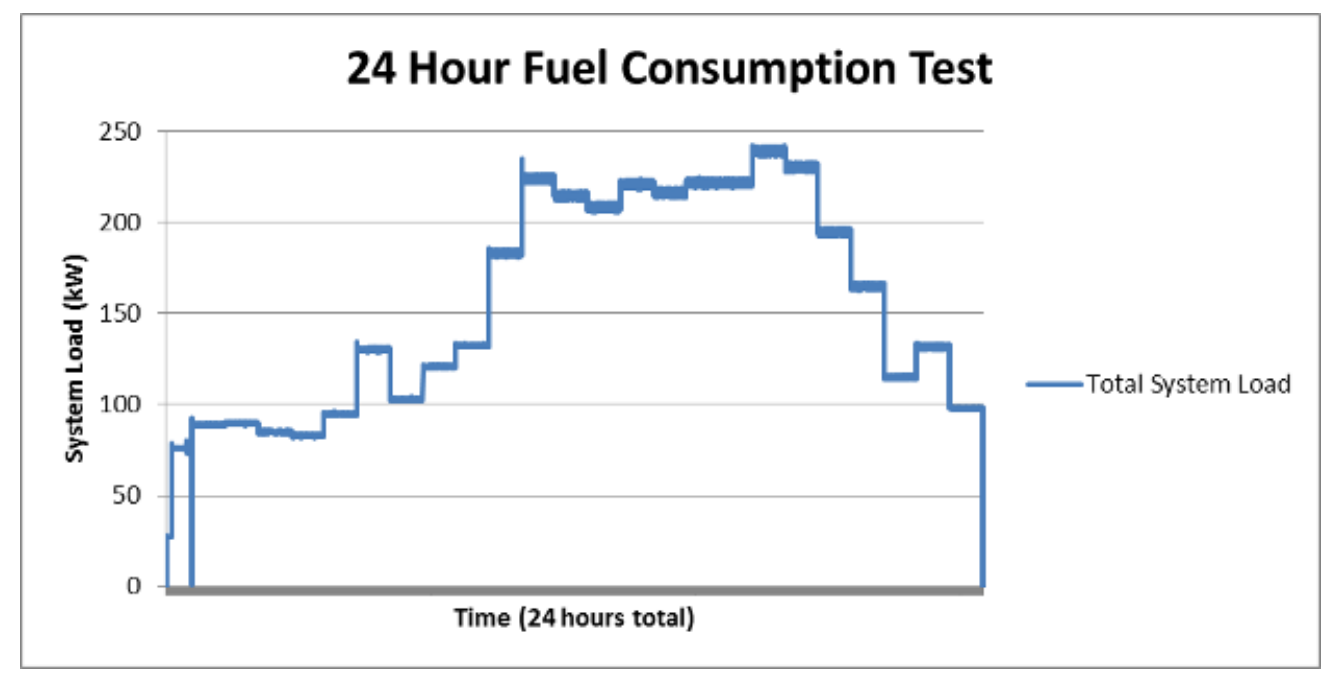

Figure E-13. 24-Hour Load Profile

The load profile for the 24-hour test is shown in Figure E-13. The test was performed February 15, 2012-February 16, 2012. This test is considered to be a success and follow-up is not needed. 


\section{Phase 2 Results}

The primary purpose of the microgrid testing was the establishment of fuel efficiency for both the microgrid and traditional spot generation and the fuel savings potential of the microgrid. The test period occurred on days with optimal solar insolation (that is, optimal PV production).

\section{E.1.2 Spot-Generation Load Profiles and Results}

The fuel and energy results with and without parasitic load for the spot-generation profiles are shown in Table E-1. The load profiles are shown in Figure E-14 through Figure E-18.

Table E-1. Spot Generation Fuel Consumption Results

\begin{tabular}{|l|r|r|r|r|r|r|}
\hline Test & $\begin{array}{r}\text { Fuel used } \\
\mathbf{w} \text { parasitic } \\
\text { load (gal) }\end{array}$ & $\begin{array}{r}\text { Energy } \\
\mathbf{w} / \text { parasitic } \\
\text { load (kWh) }\end{array}$ & $\begin{array}{r}\text { Efficiency } \\
\mathbf{w} \text { /parasitic } \\
\text { load (gal/kWh) }\end{array}$ & $\begin{array}{r}\text { Fuel used } \\
\mathbf{w} / \text { o parasitic } \\
\text { load (gal) }\end{array}$ & $\begin{array}{r}\text { Energy } \\
\text { w/o parasitic } \\
\text { load (kWh) }\end{array}$ & $\begin{array}{r}\text { Efficiency } \\
\text { w/o parasitic } \\
\text { load (gal/kWh) }\end{array}$ \\
\hline Billet & 153 & 1,722 & 0.089 & 75 & 751 & 0.099 \\
\hline Kitchen & 78 & 861 & 0.091 & 75 & 750 & 0.099 \\
\hline Latrine & 43 & 243 & 0.175 & 32 & 140 & 0.225 \\
\hline Shower & 53 & 416 & 0.127 & 47 & 306 & 0.154 \\
\hline Laundry & 54 & 451 & 0.120 & 50 & 342 & 0.146 \\
\hline Total: & 381 & 3,692 & 0.103 & 352 & 3,039 & 0.116 \\
\hline
\end{tabular}

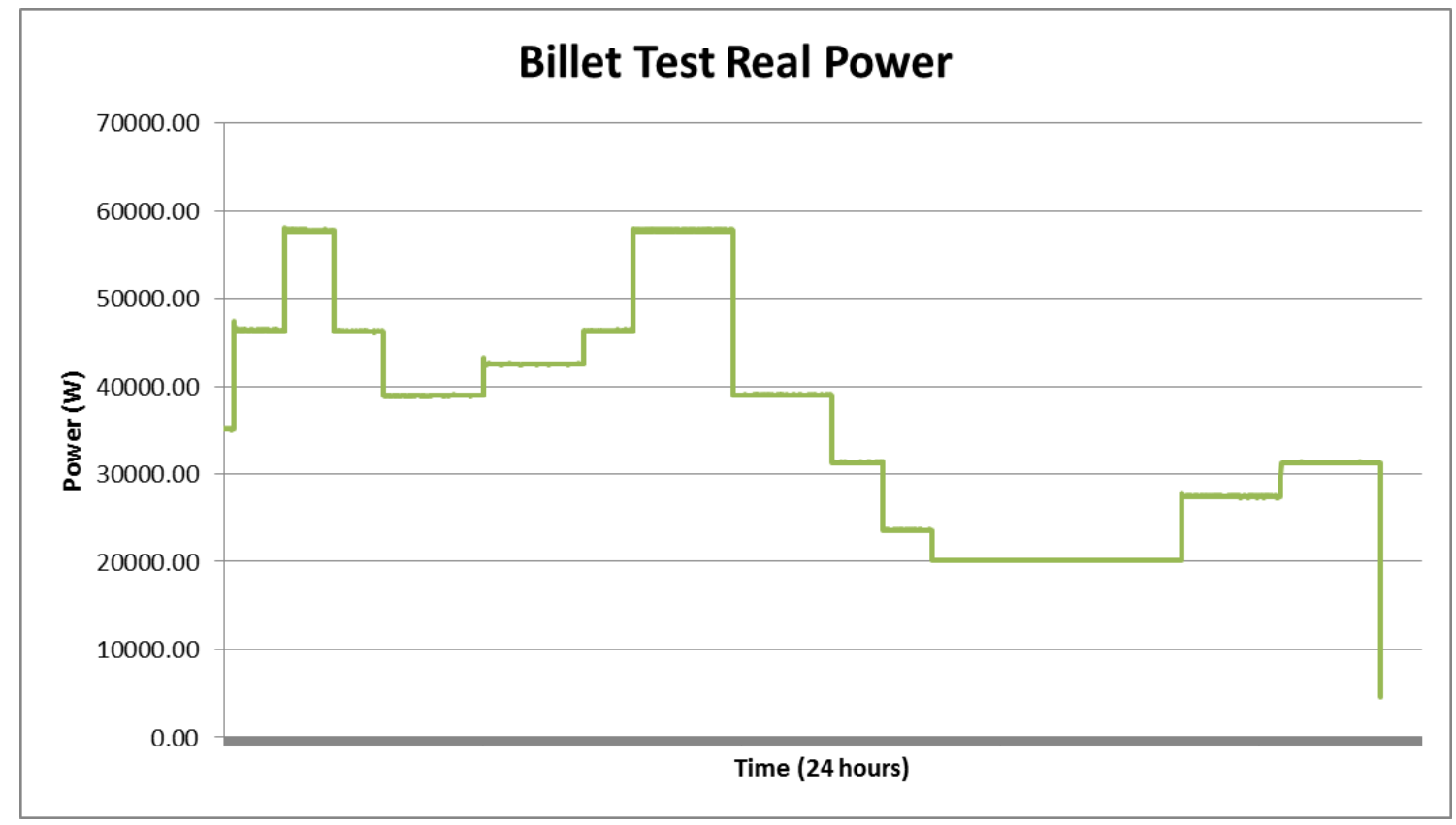

Figure E-14. Spot-Generation Billet Profile 


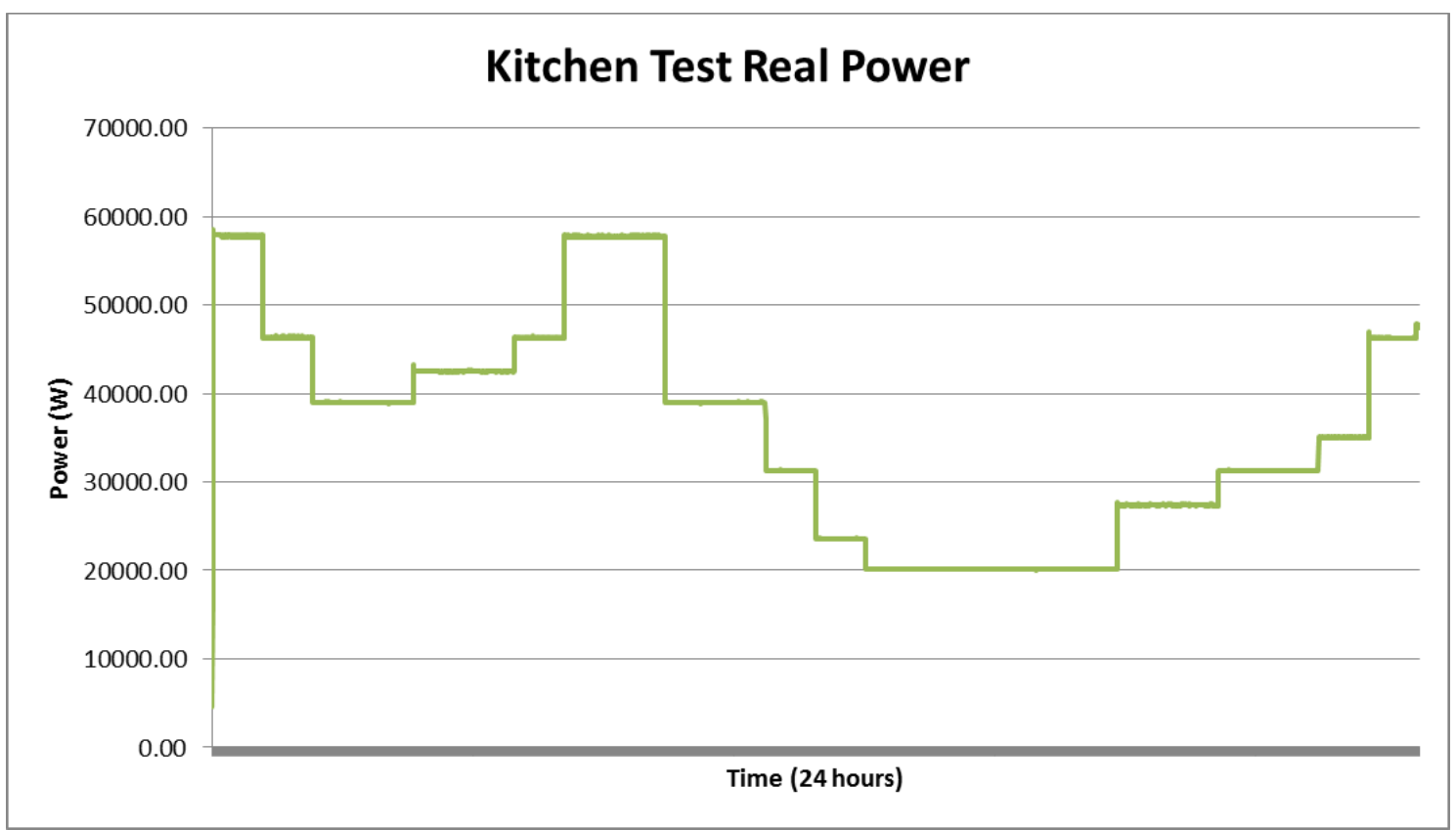

Figure E-15. Spot-Generation Kitchen Profile

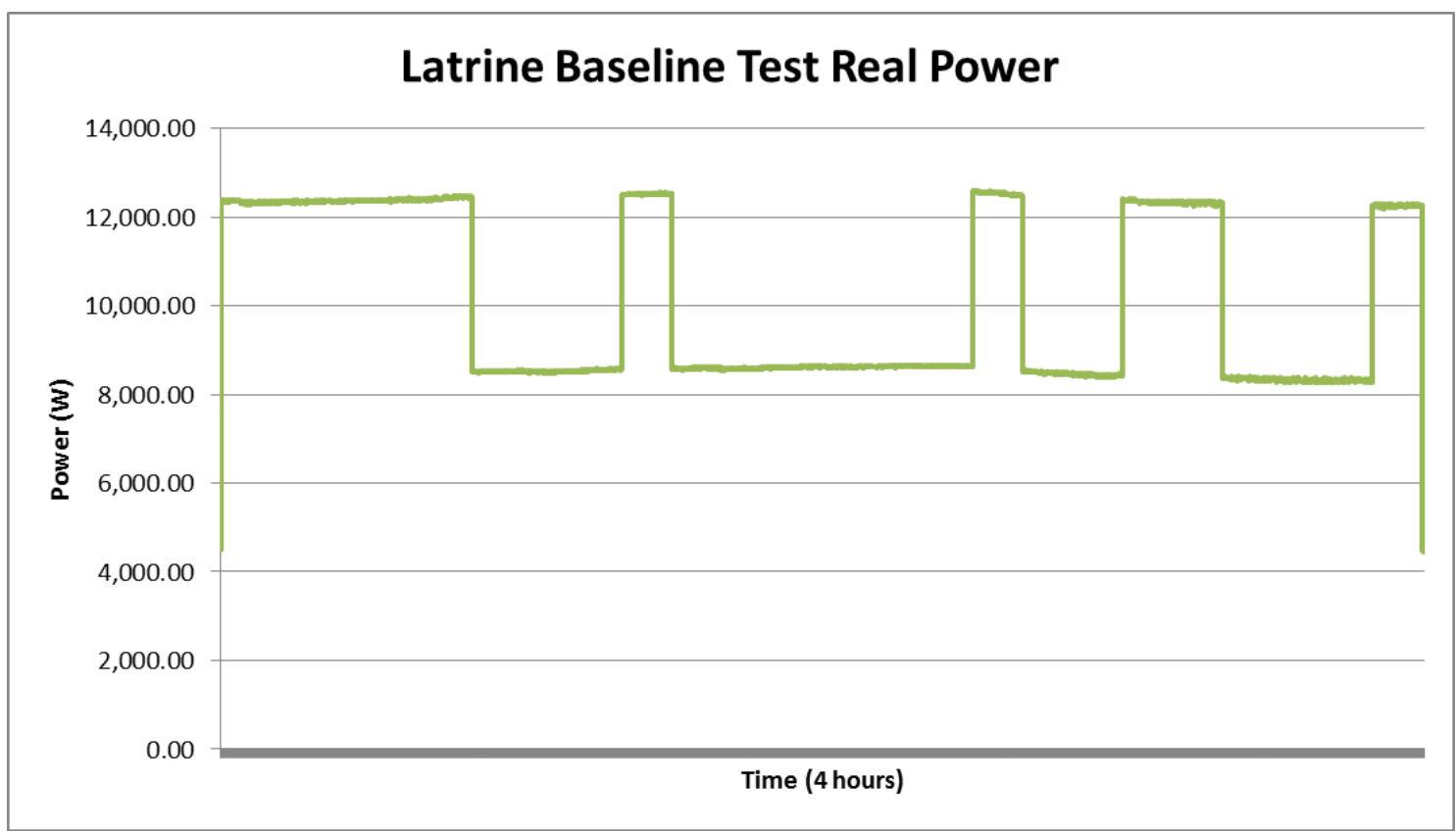

Figure E-16. Spot-Generation Latrine Profile 


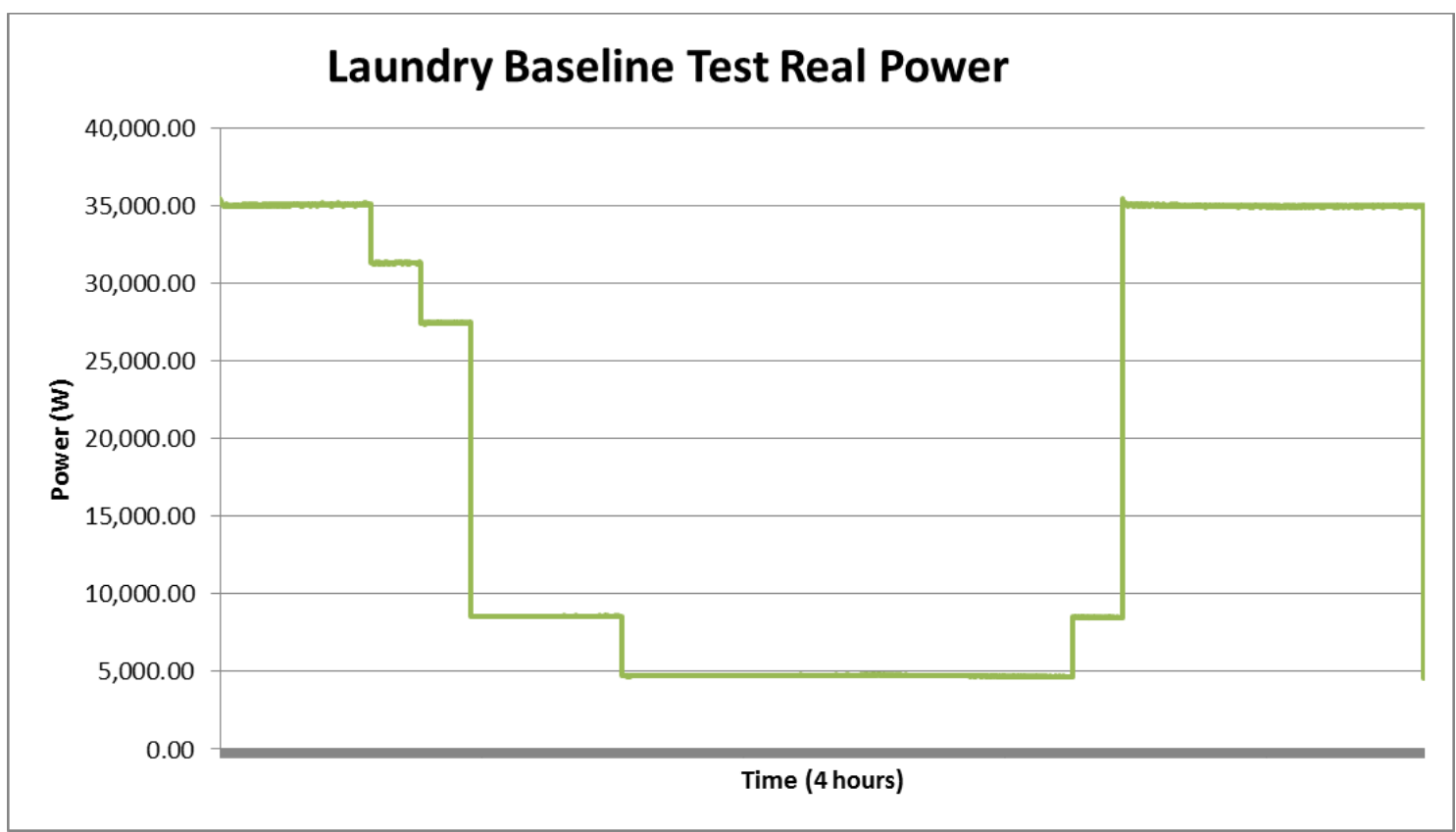

Figure E-17. Spot-Generation Laundry Profile

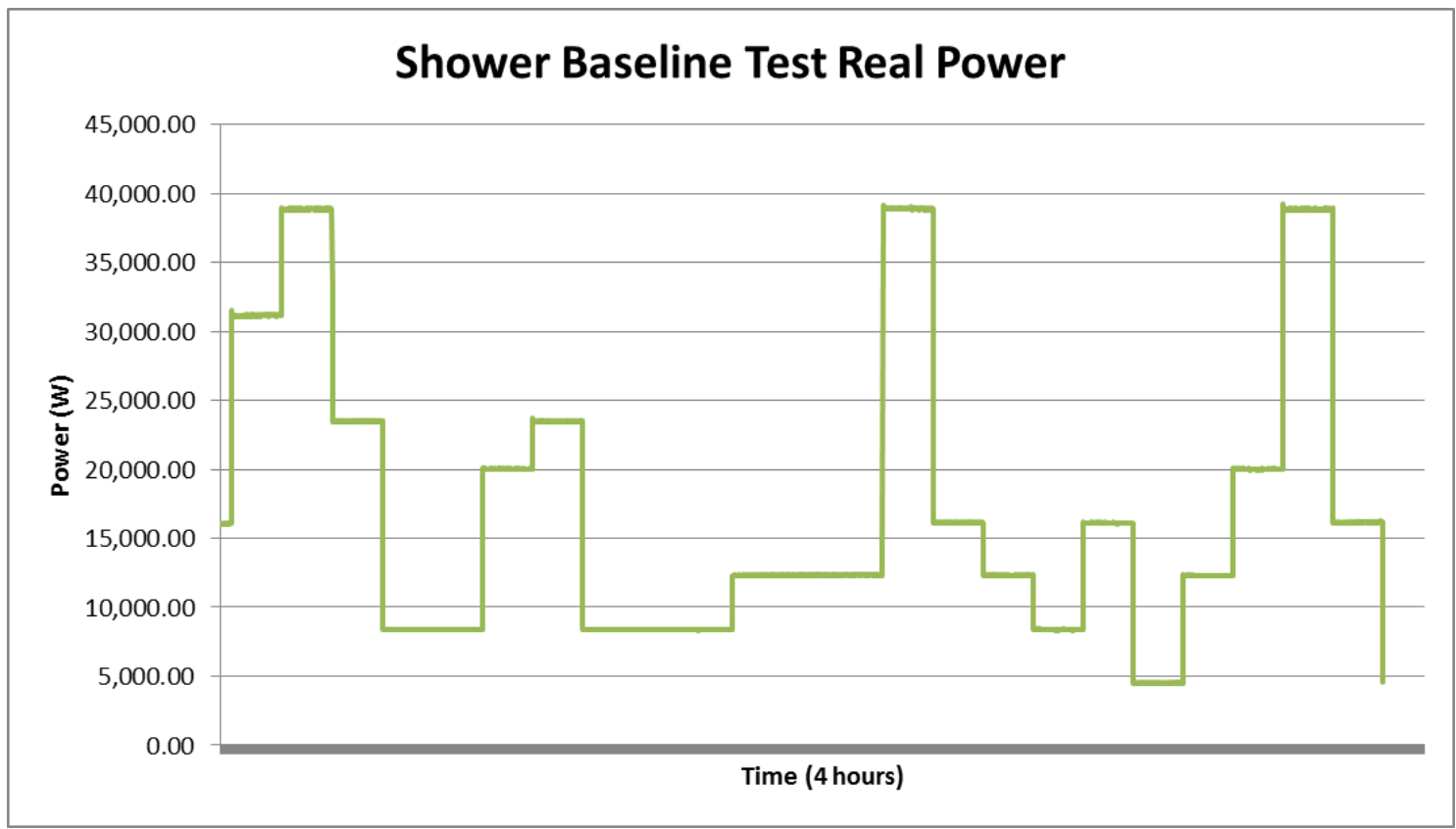

Figure E-18. Spot-Generation Shower Profile

\section{E.1.3 Microgrid Load Profile and Results}

The fuel and energy results with and without parasitic load for the microgrid profiles are shown in Table E-2. The results for the 24-hour profile with a full PV (all shelters) were calculated because the SAGE installation at BCIL has only two PV arrays installed (each array is sized to be roughly equivalent to a shelter's electrical load). Further, the PV contributions are 
assumed to be best case, on a sunny day ( $95 \mathrm{kWh}$ for two panels). The load profiles are shown in Figure E-19 and Figure E-20.

Table E-2. Microgrid Results

\begin{tabular}{lcccccc}
\hline & $\begin{array}{c}\text { Fuel Used } \\
\text { w/Parasitic } \\
\text { Load } \\
\text { (gal) }\end{array}$ & $\begin{array}{c}\text { Energy } \\
\text { w/Parasitic } \\
\text { Load } \\
\text { ThWh) }\end{array}$ & $\begin{array}{c}\text { Efficiency } \\
\text { w/Parasitic } \\
\text { Load } \\
\text { (gal/kWh) }\end{array}$ & $\begin{array}{c}\text { Fuel Used } \\
\text { w/o } \\
\text { Parasitic } \\
\text { Load } \\
\text { (gal) }\end{array}$ & $\begin{array}{c}\text { Energy } \\
\text { w/o } \\
\text { Parasitic } \\
\text { Load } \\
\text { (kWh) }\end{array}$ & $\begin{array}{c}\text { Efficiency } \\
\text { w/o } \\
\text { Parasitic } \\
\text { Load } \\
\text { (gal/kWh) }\end{array}$ \\
\hline $\begin{array}{l}\text { 24-Hour } \\
\text { Profile w/o } \\
\text { PV }\end{array}$ & 284.31 & $3,290.26$ & 0.08641 & 274.98 & $3,182.26$ & 0.08833 \\
$\begin{array}{l}\text { 24-Hour } \\
\text { Profile with } \\
\text { Single PV }\end{array}$ & 269.89 & $3,295.63$ & 0.08189 & 261.05 & $3,187.63$ & 0.08189 \\
$\begin{array}{l}\text { 24-Hour } \\
\text { Profile with } \\
\text { Full PV }\end{array}$ & N/A & N/A & & & & \\
\hline
\end{tabular}

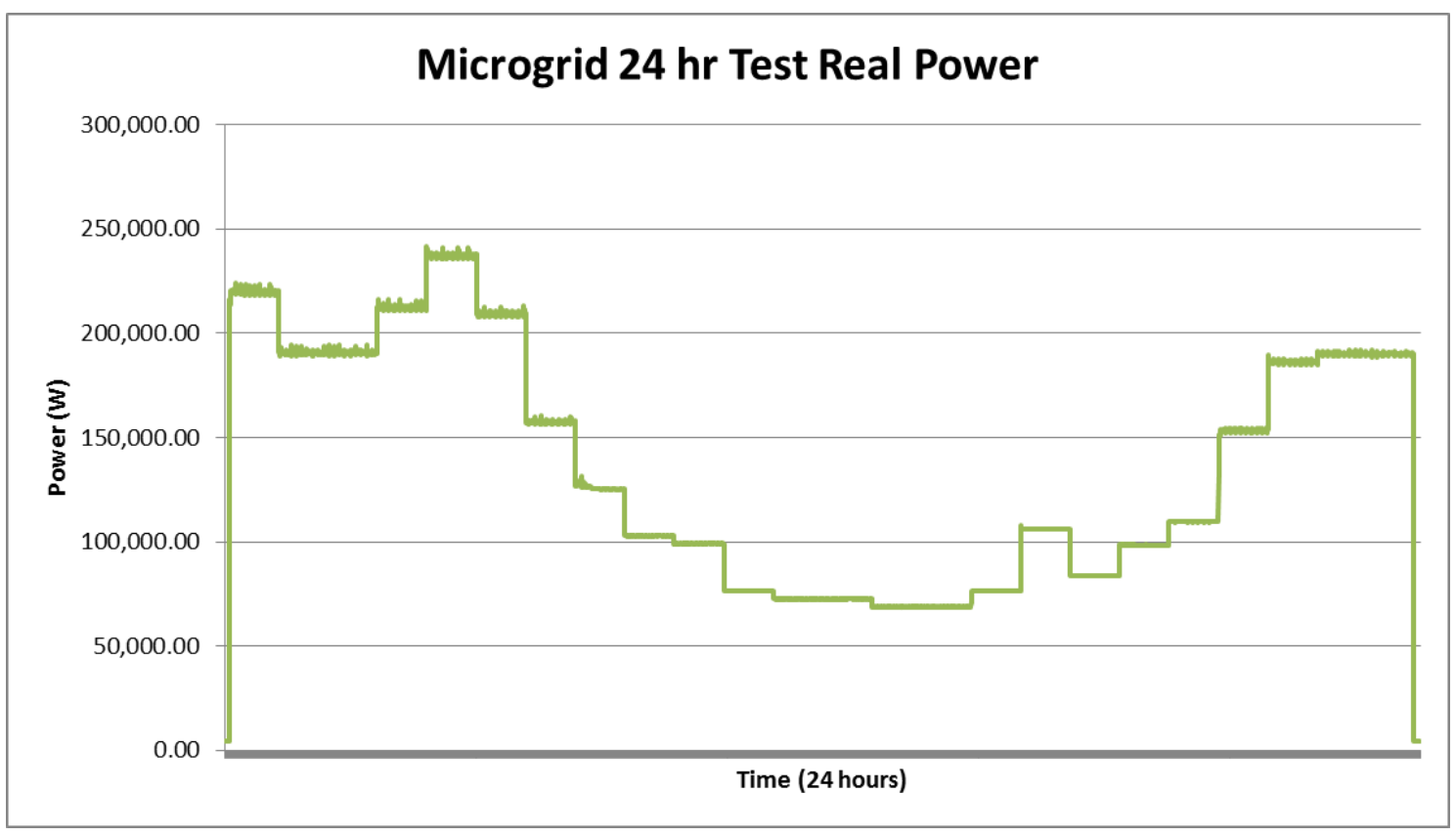

Figure E-19. Microgrid 24-Hour Load Profile (without PV) 


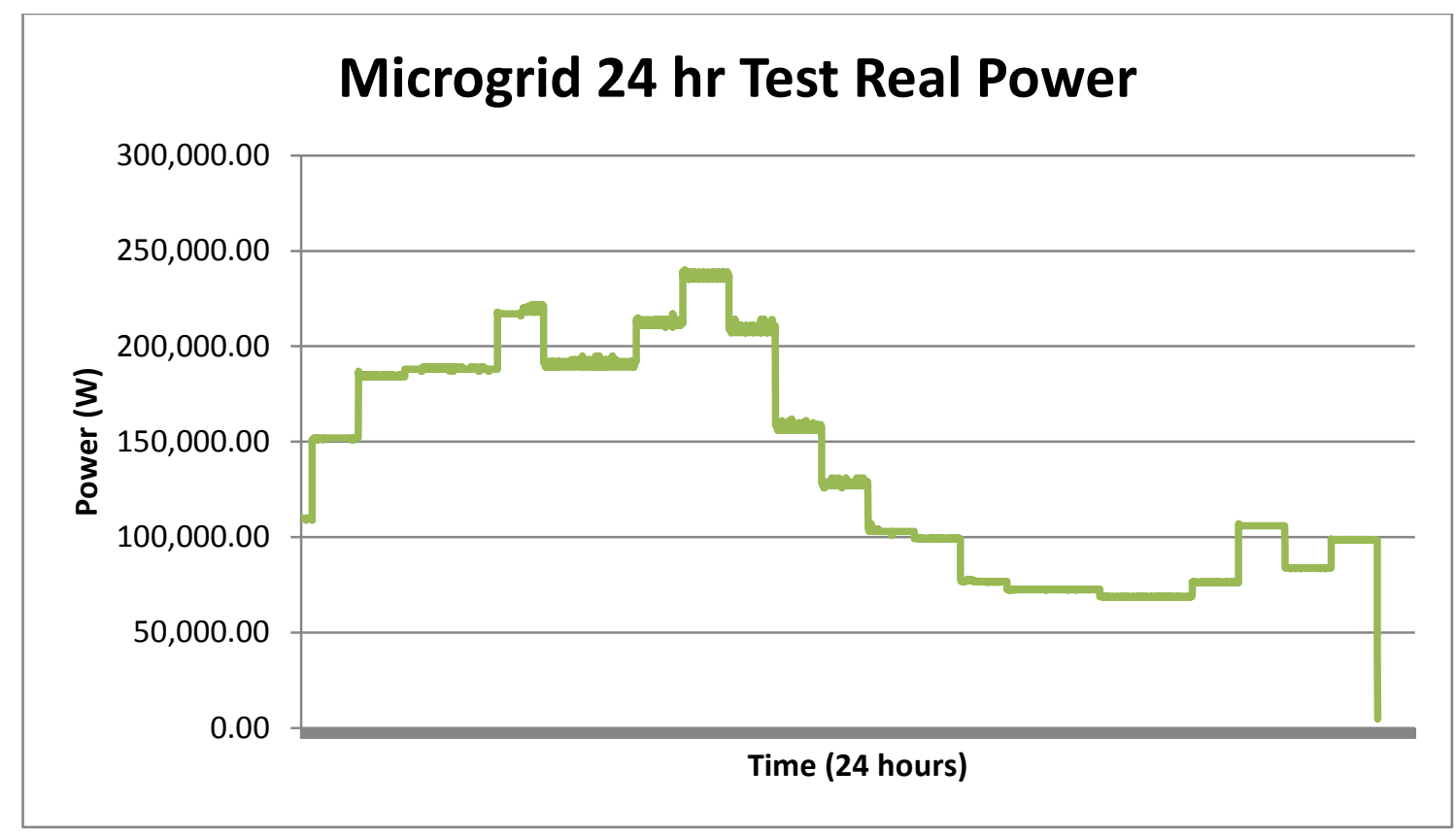

Figure E-20. Microgrid 24-Hour Load Profile (with Single PV)

\section{E.1.3.1 Stability and Reliability}

The microgrid performed as expected. Grid stability is typically evaluated by comparing voltage and/or frequency deviations from nominal values against an acceptable tolerance. No frequency or voltage stability problems were noted. The plots of voltage and frequency for both the microgrid and spot-generation tests are shown in Figure E-21 through Figure E-34. Voltage and frequency variations were within allowable specifications. No generation outages took place. However, the ground-mounted fuel pump that supplies each generator with fuel broke down (as a result of being run dry) and was replaced. Additionally, Generator 1 had to have its Engine Management and Control Panel (EMCP) 4.4 unit replaced. The extremes shown on the left of any graph represent the test startup.

\section{$\underline{\text { Silent Run }}$}

The silent run capability of the battery system was evaluated against a $100 \mathrm{~kW}$ load and a $250 \mathrm{~kW}$ load. The system performed slightly better than expected. Runtime with a $100 \mathrm{~kW}$ load was 2 hours and 14 minutes and runtime with a $250 \mathrm{~kW}$ load was $26 \mathrm{~min}$.

Figure E-21 through Figure E-30 show power quality quantities (voltage and frequency) of spot generation tests. Figure E-31 through Figure E-34 show power quality quantities (voltage and frequency) of microgrid tests, which demonstrate the stable and reliable operation of the microgrid despite several major load fluctuations built into the load profiles. 


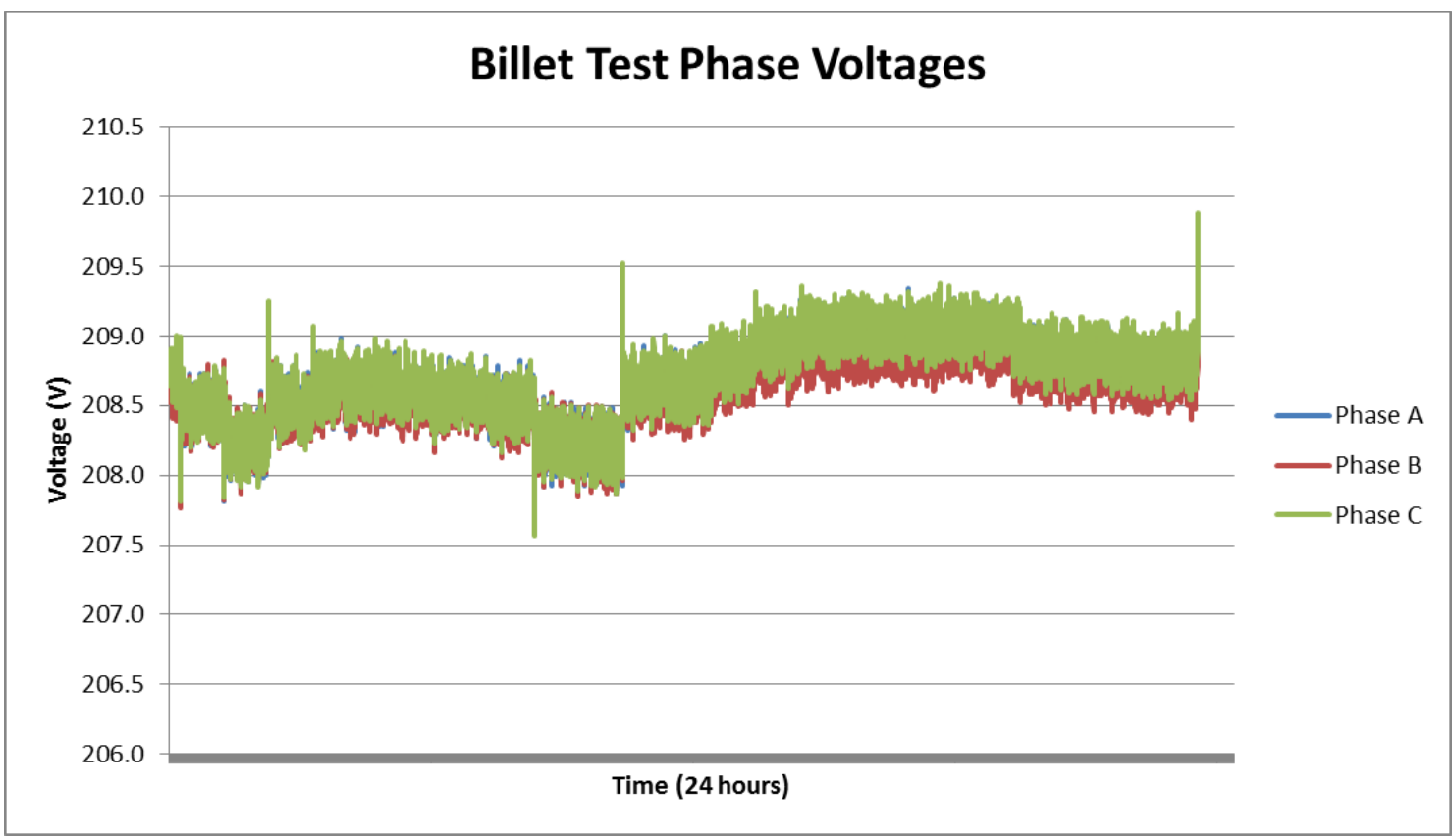

Figure E-21. Spot-Generation Billet Test Voltages

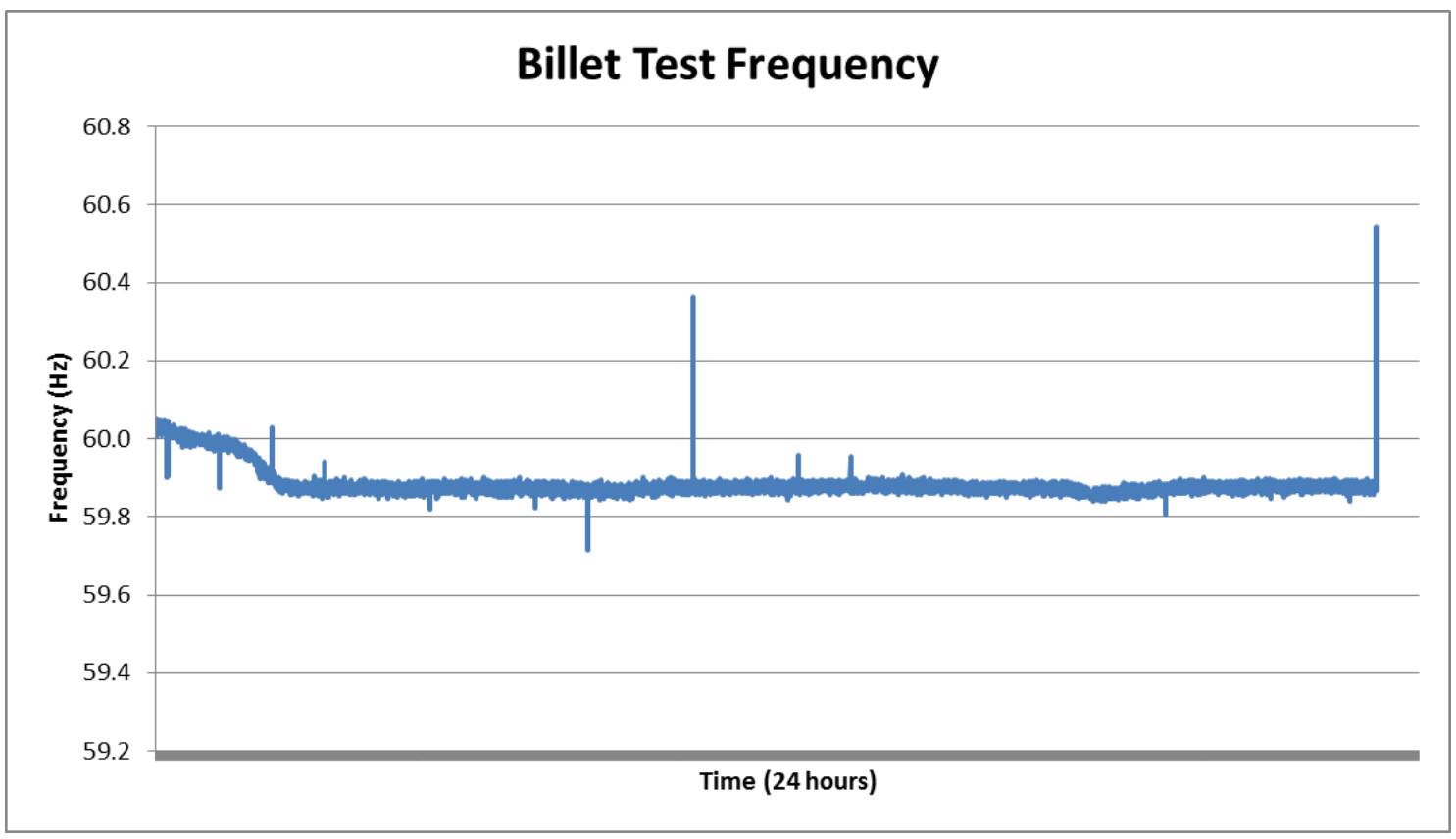

Figure E-22. Spot-Generation Billet Test Frequency 


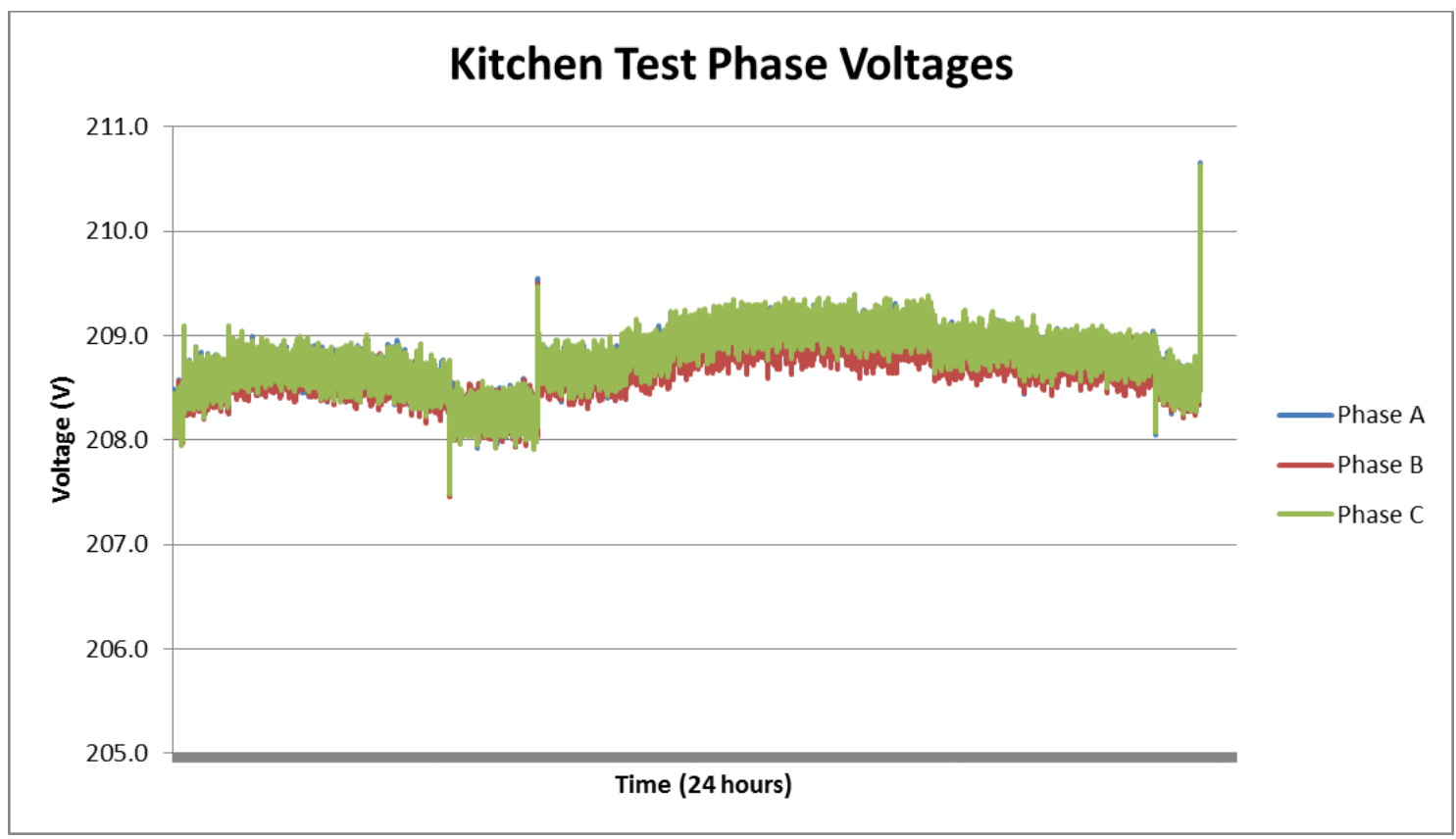

Figure E-23. Spot-Generation Kitchen Test Voltages

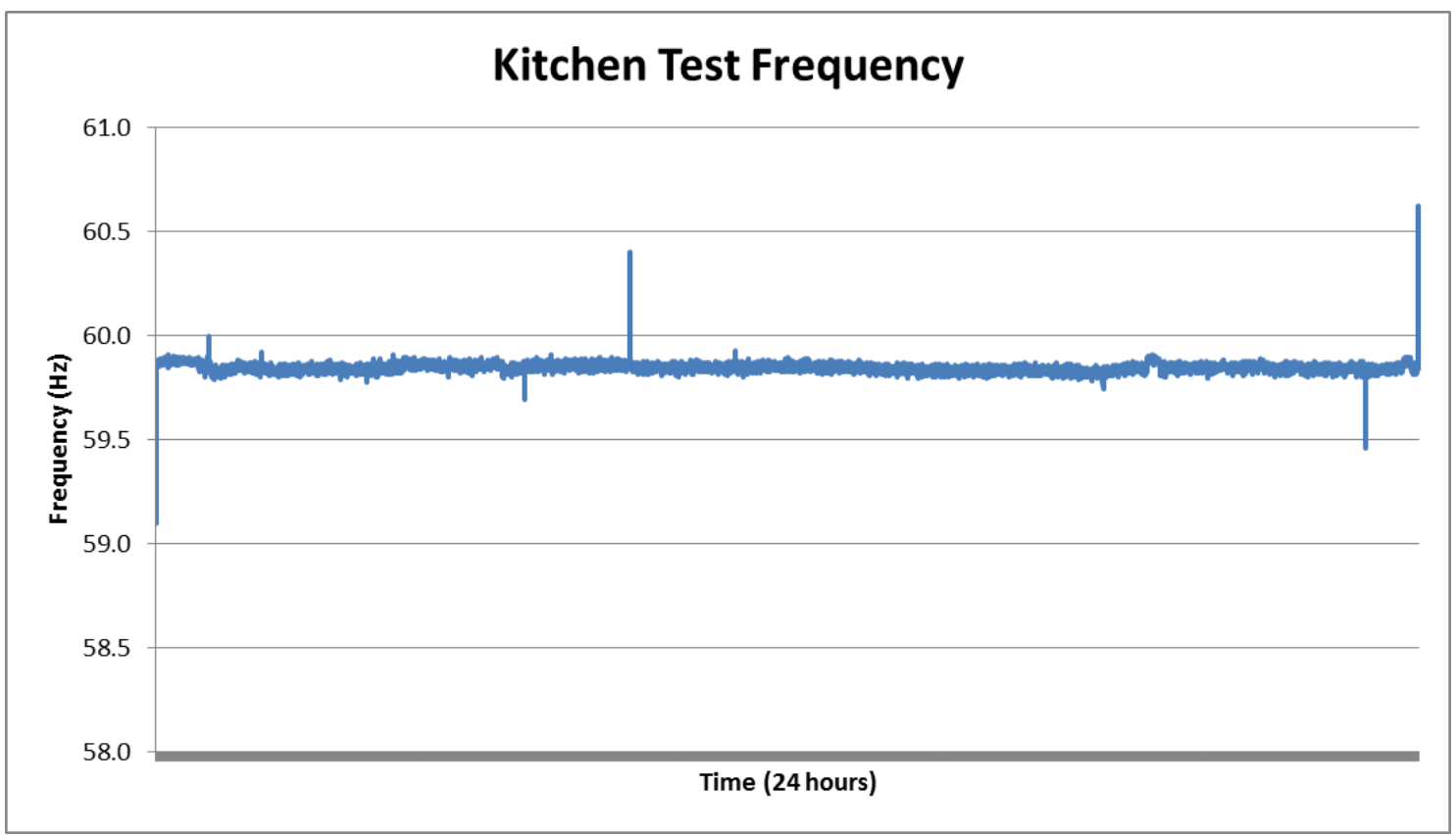

Figure E-24. Spot-Generation Kitchen Test Frequency 


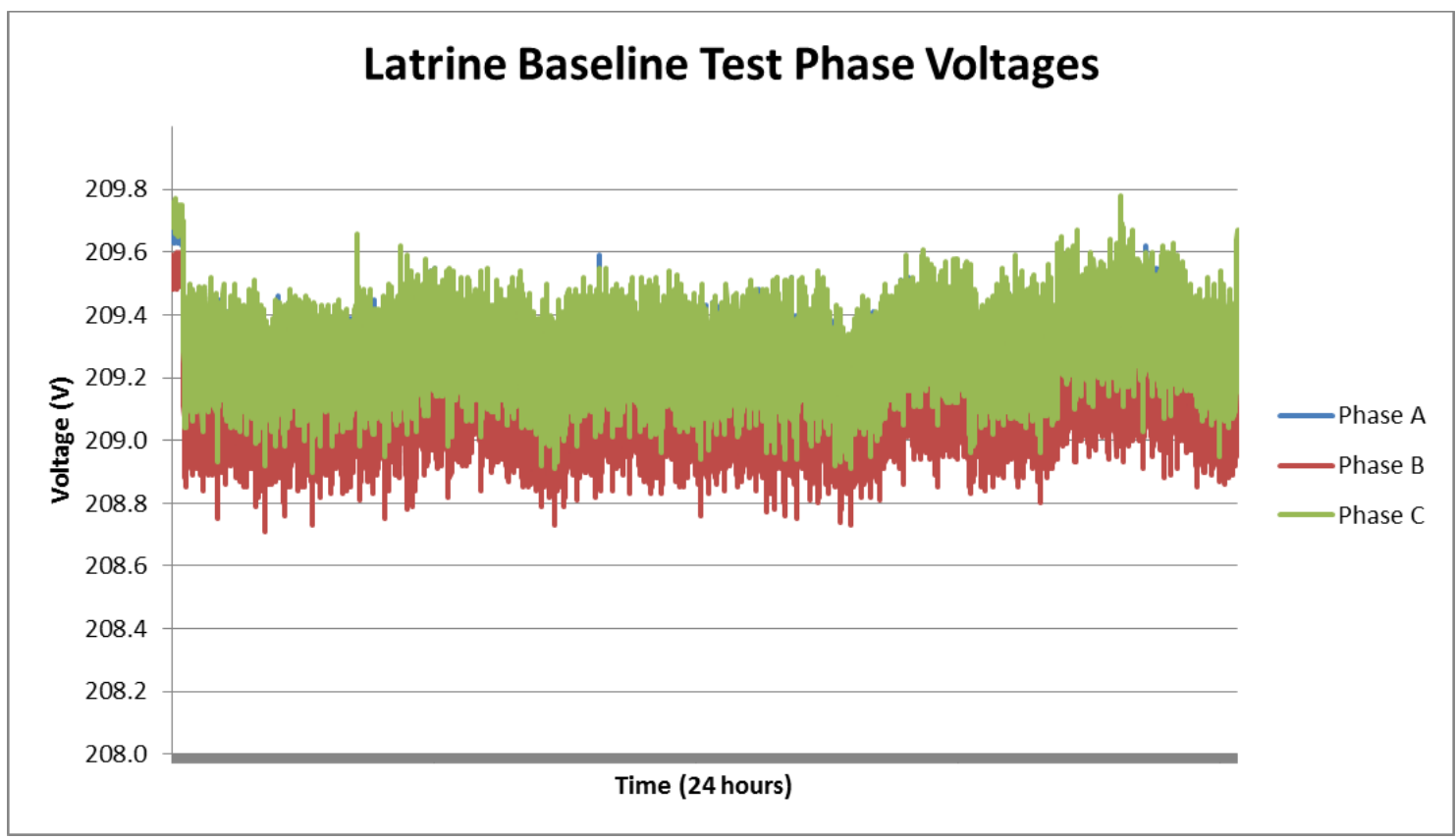

Figure E-25. Spot-Generation Latrine Test Voltages

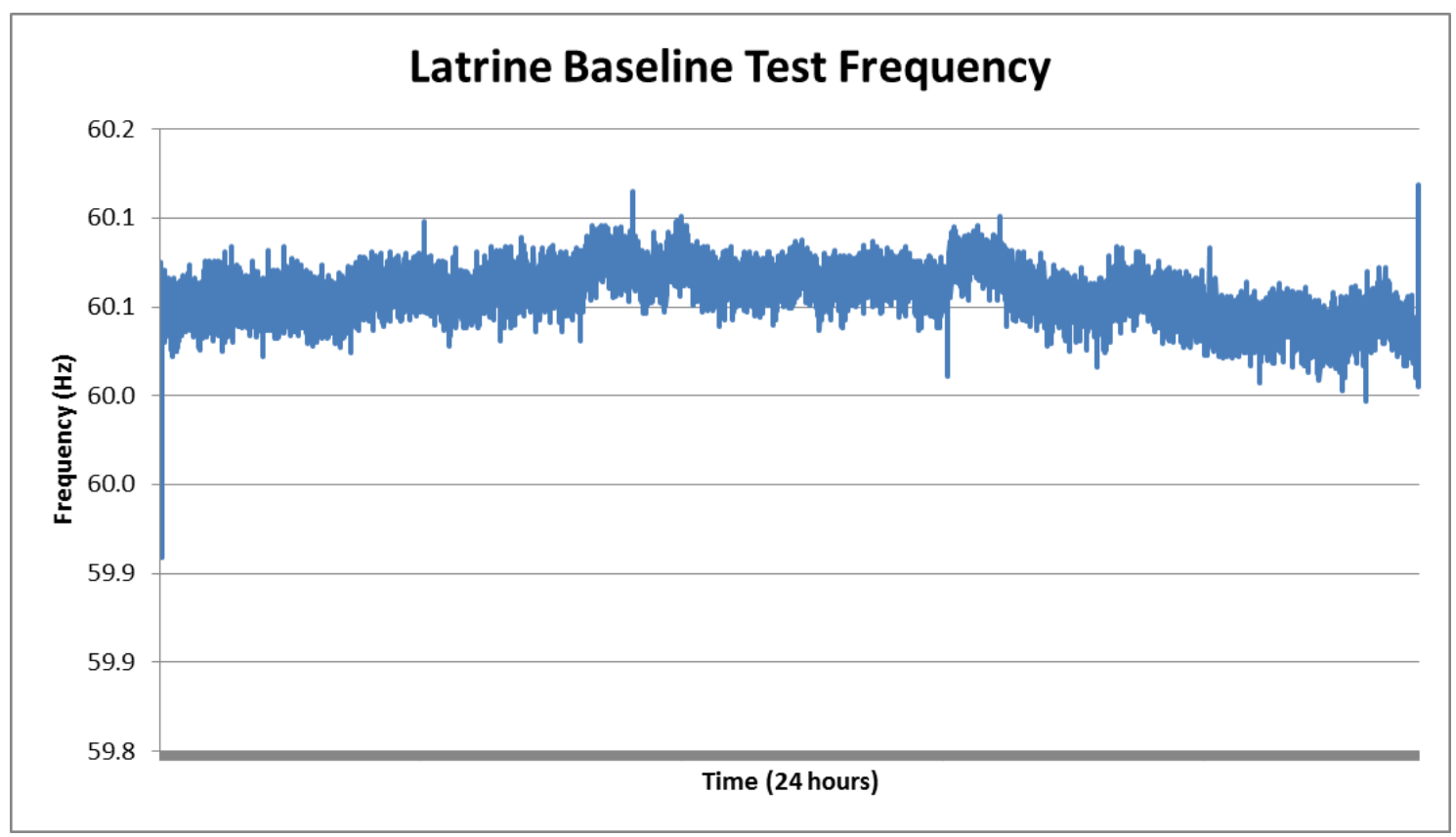

Figure E-26. Spot-Generation Latrine Test Frequency 


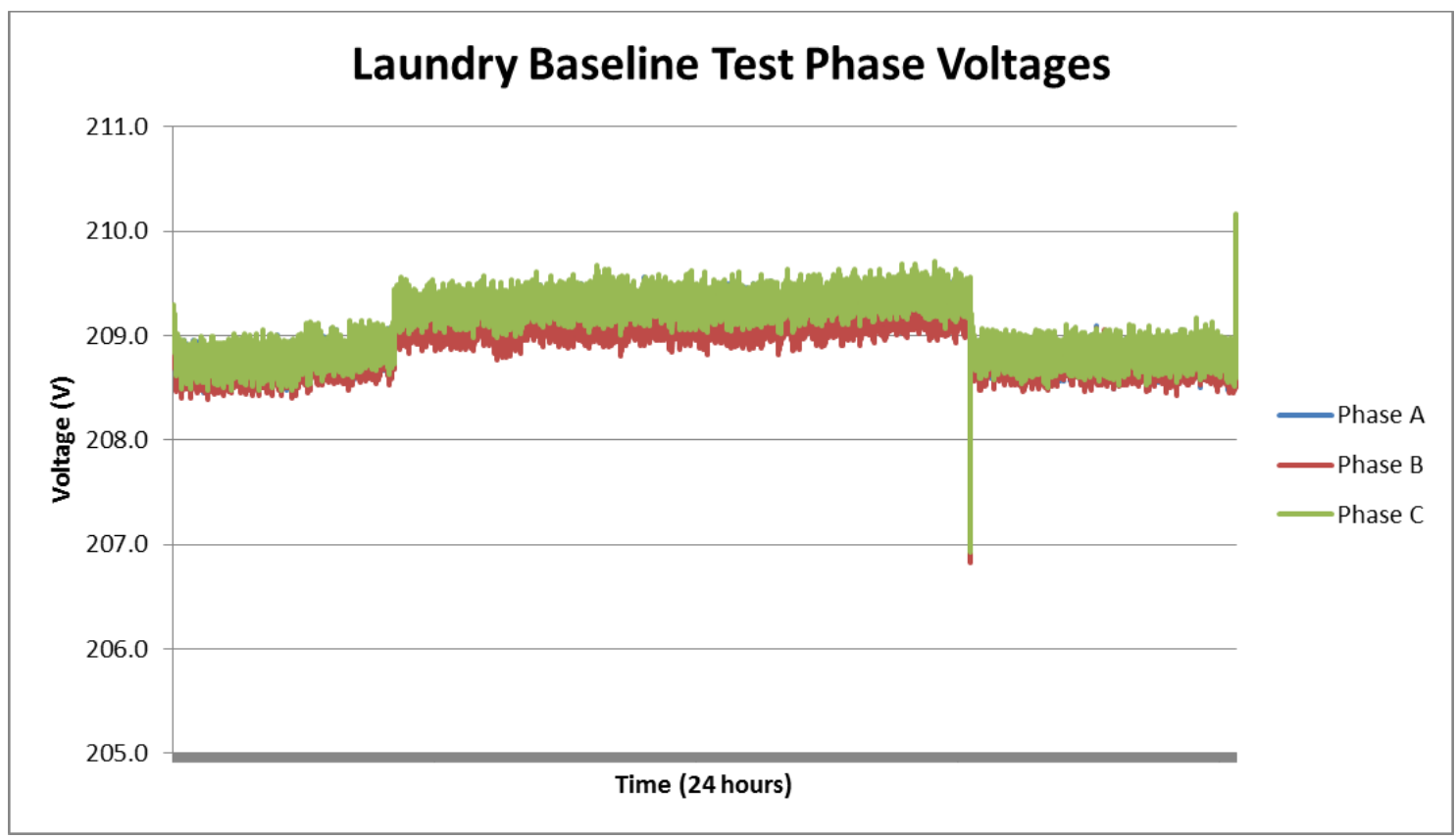

Figure E-27. Spot-Generation Laundry Test Voltages

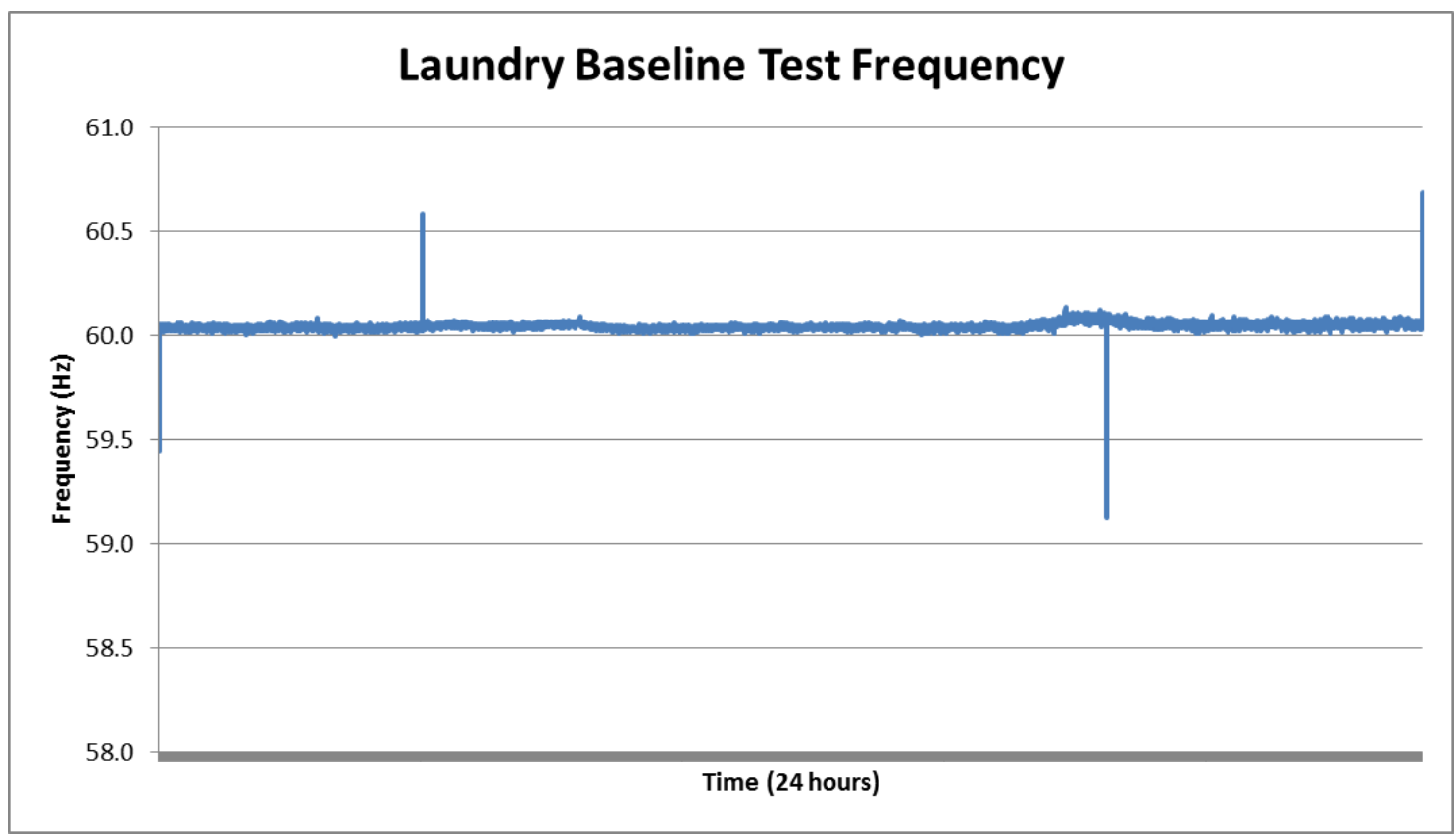

Figure E-28. Spot-Generation Laundry Test Frequency 


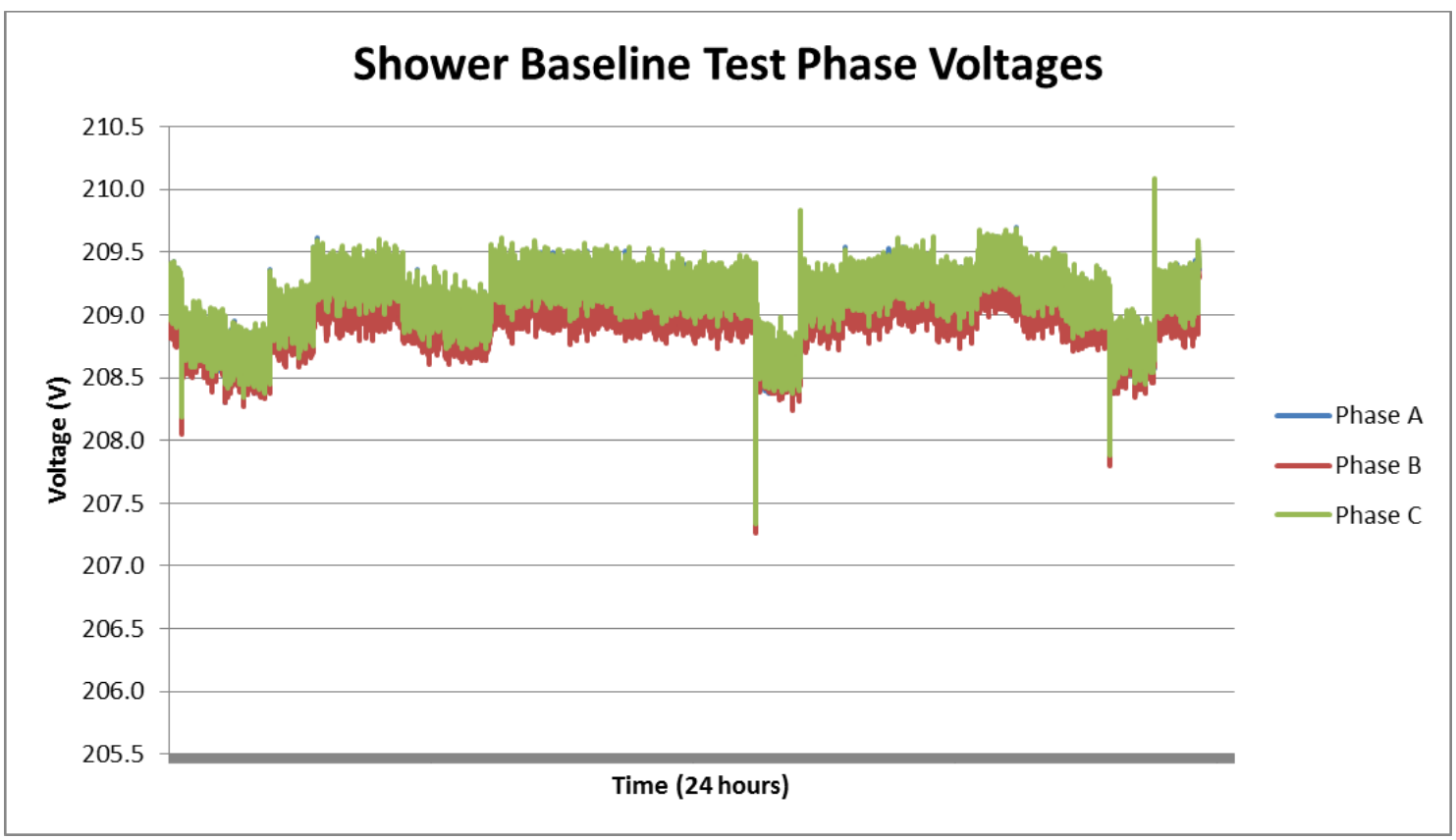

Figure E-29. Spot-Generation Shower Test Voltages

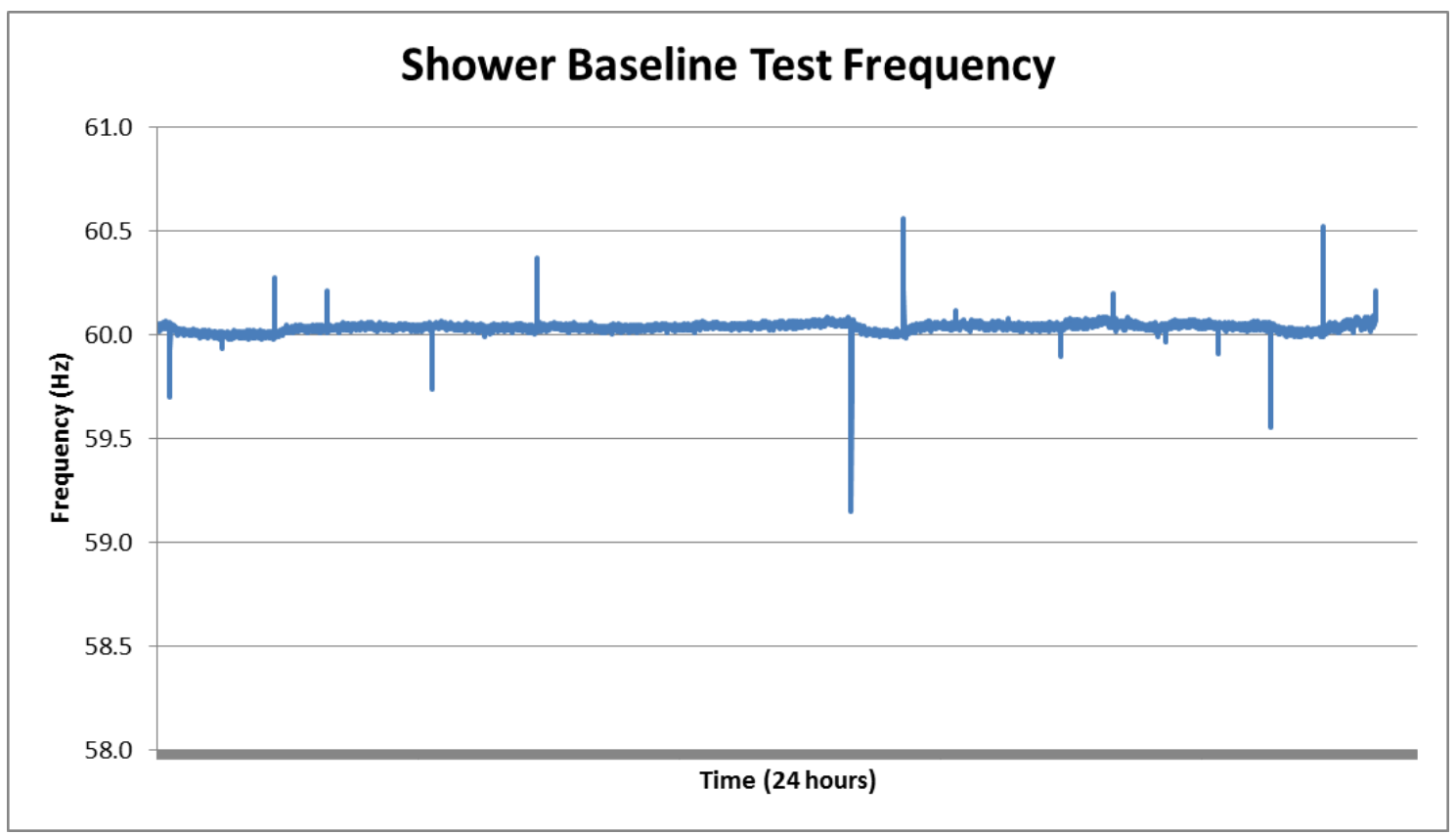

Figure E-30. Spot-Generation Shower Test Frequency 


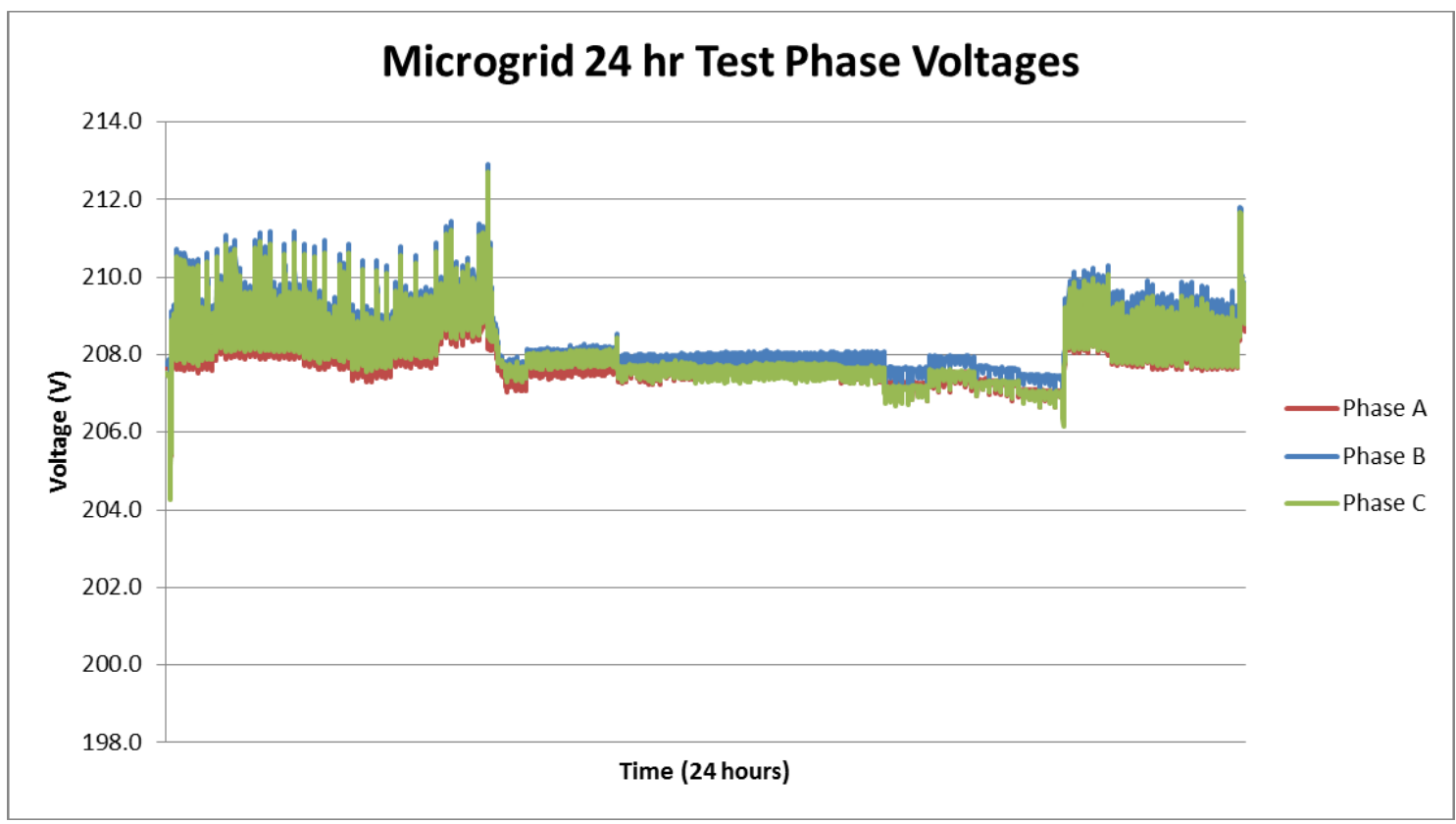

Figure E-31. Microgrid 24-Hour Test Phase Voltages (No PV)

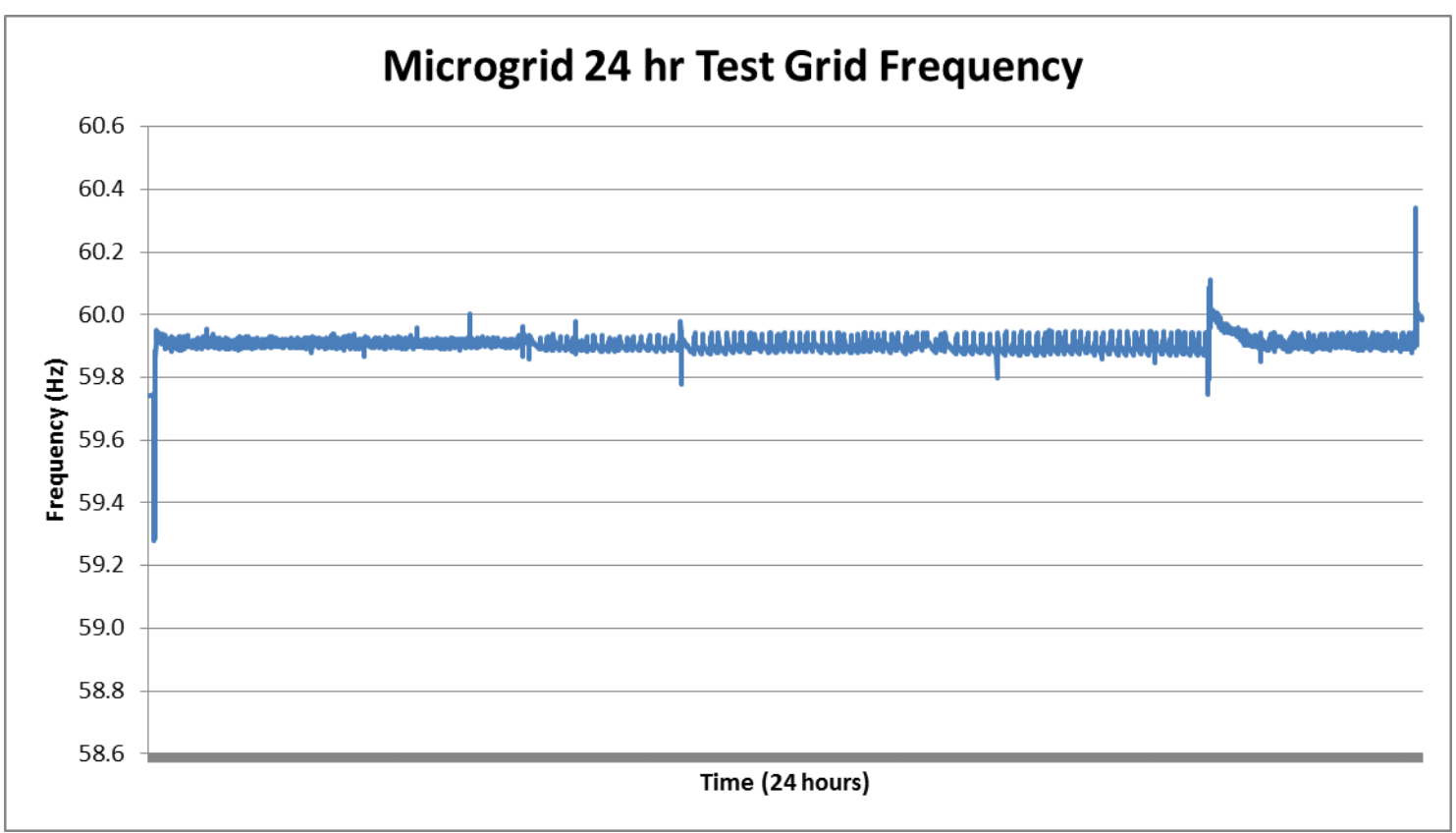

Figure E-32. Microgrid 24-Hour Test Frequency (No PV) 


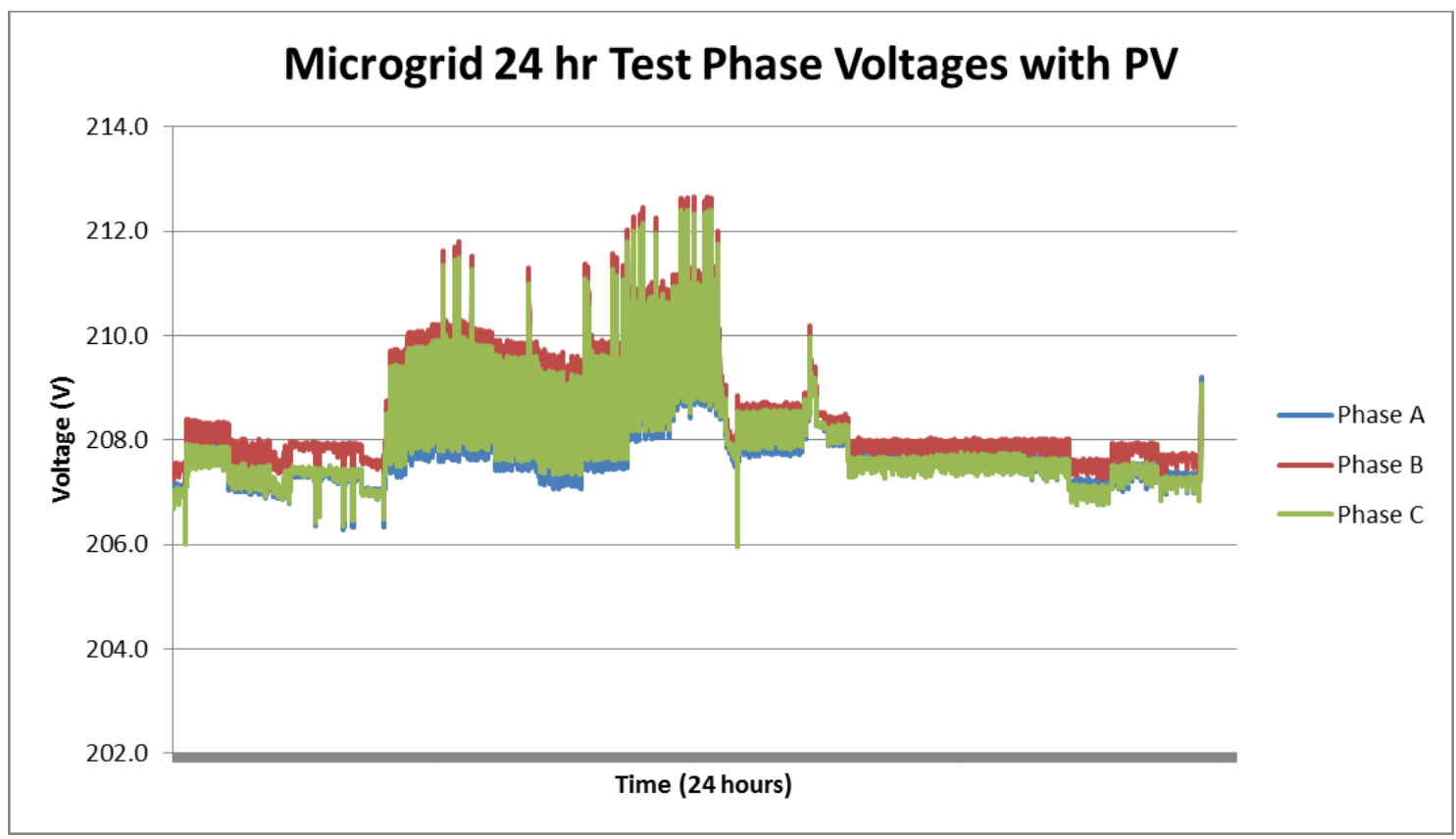

Figure E-33. Microgrid 24-Hour Test Voltages (with Single PV)

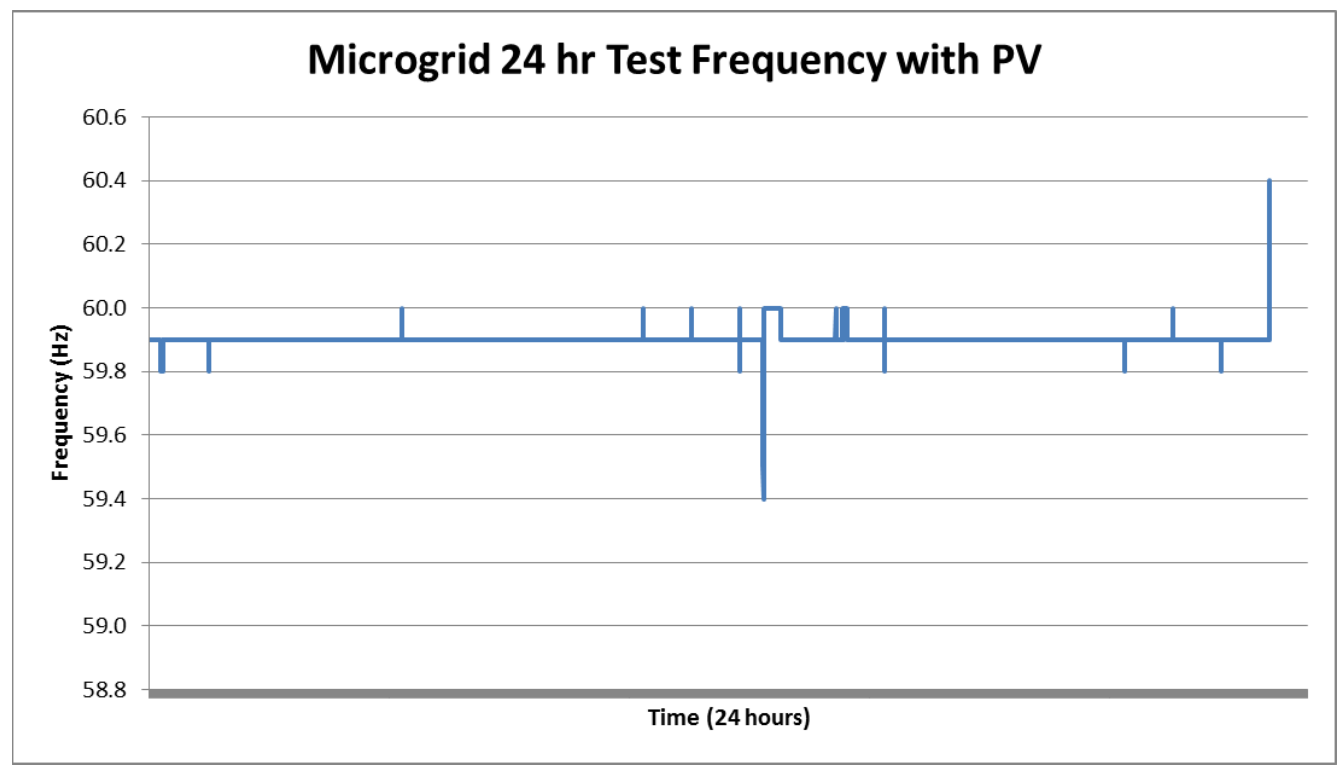

Figure E-34. Microgrid 24-Hour Test Frequency (with Single PV)

\section{E.1.3.2 Photovoltaic Array Testing}

The photovoltaic array performed as expected and properly synchronized to the microgrid as well as the actual grid. The PV system was connected to the microgrid only for PV-specific tests. At any other time, BCIL connected the PV system to the main electric grid to feed power back to the utility. Peak power output was observed to be $13 \mathrm{~kW}$ (rated $12.9 \mathrm{~kW}$ ), and peak energy output for any 24-hour period was observed to be $96.0 \mathrm{kWh}$. This is considered to be a best-case scenario. 


\section{E.1.3.3 Energy Efficient Shelter Testing}

The results of this test were rendered unusable through unreliable instrumentation. The testing will be repeated, albeit with actual troop occupancy, during Phase 3 in October 2012.

\section{E.1.3.4 DSOM Testing}

The power levels for the HVAC system (Mitsubishi heat pump), while somewhat stable during testing, cannot be considered static. The HVAC system uses a variable-level heat pump to change load level based on the temperature differential between inside and outside air. The results are shown in Table E-3, including the observed HVAC power levels.

Table E-3. Shelter Load Levels

\begin{tabular}{lc}
\hline \multicolumn{1}{c}{ Load } & Load Level \\
\hline Blue LED & $40 \mathrm{~W}$ \\
White LED & $80 \mathrm{~W}$ \\
Cooling & $1050 \mathrm{~W}$ \\
Heating & $1450 \mathrm{~W}$ \\
\hline
\end{tabular}

\section{Phase 3 Results}

\section{E.1.4 Microgrid Testing}

At 10:07 a.m. on October 18, 2012, the entire north camp was switched from shore power to microgrid power. The PV system was configured to supply power (when available) to the microgrid. Data was collected via the Caterpillar data collection system, from the following components; all north camp Shark power meters and DSOM. Fuel use was monitored via a calibrated FTI flow meter installed at the central north camp storage tank. The plots for total camp power from generators and battery system over 72 hours are shown in Figure E-35. 


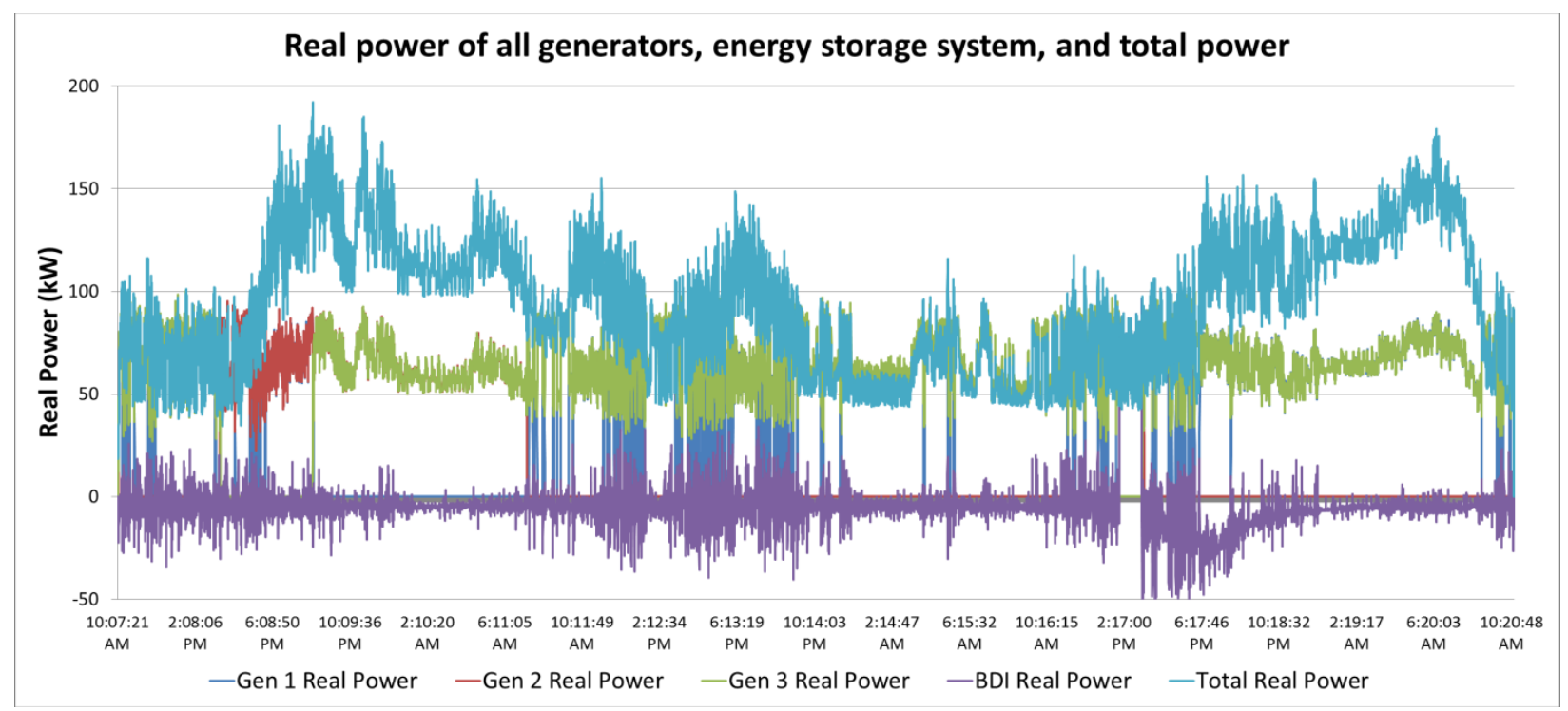

Figure E-35. Power Profiles for Generators and Energy Storage System

\section{E.1.4.1 Stability and Reliability}

The microgrid performed as expected. Grid stability is typically measured by voltage and/or frequency deviations outside of an acceptable tolerance. No frequency or voltage stability problems were noted, except for a drop in grid frequency during the silent-run mode operation. This is somewhat expected due to the drop control integrated into the bidirectional inverter firmware and the large camp load. The plot for microgrid frequency is shown in Figure E-36. Voltage and frequency variations were within allowable specifications. No generation outages took place.

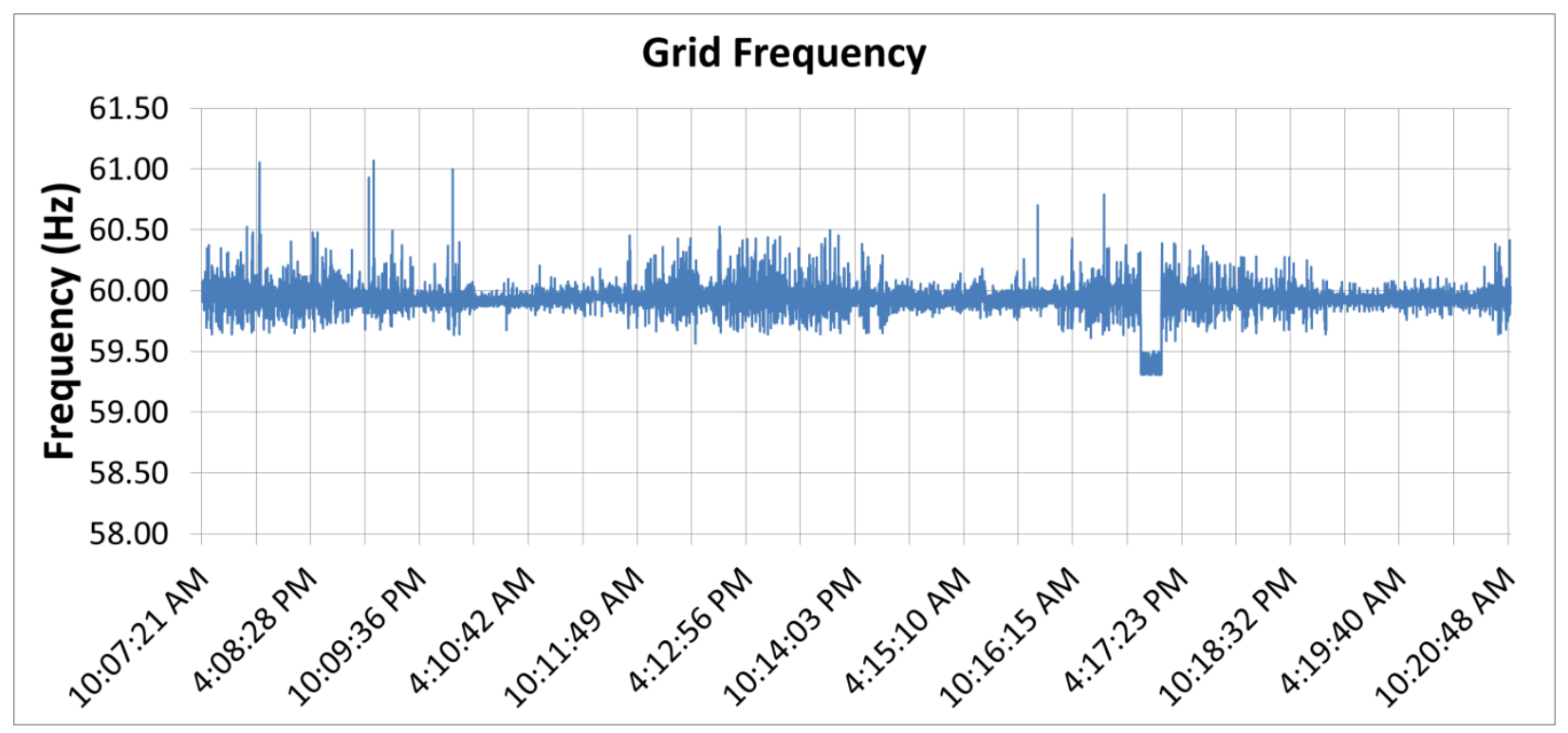

Figure E-36. Microgrid Frequency for 72-Hour Test 


\section{E.1.4.2 Silent Run}

The system was switched to silent-run mode for approximately 30 minutes on the afternoon of Saturday, October 20, 2012. The goal of this test was to establish that the microgrid could indeed power the entire camp load for a short time. No metrics other than already monitored grid parameters were recorded. A notable observation was the drop in grid frequency during the test, as shown in Figure E-36. The drop is attributed to the droop control integrated into the bidirectional inverter firmware for load sharing. The camp load at test time was large enough to cause the noticeable frequency drop when the bidirectional inverter ran the entirety of the camp load by itself. The frequency deviation was well within the specification of 59.3-60.7 Hz. The battery system state of charge is shown in Figure E-37.

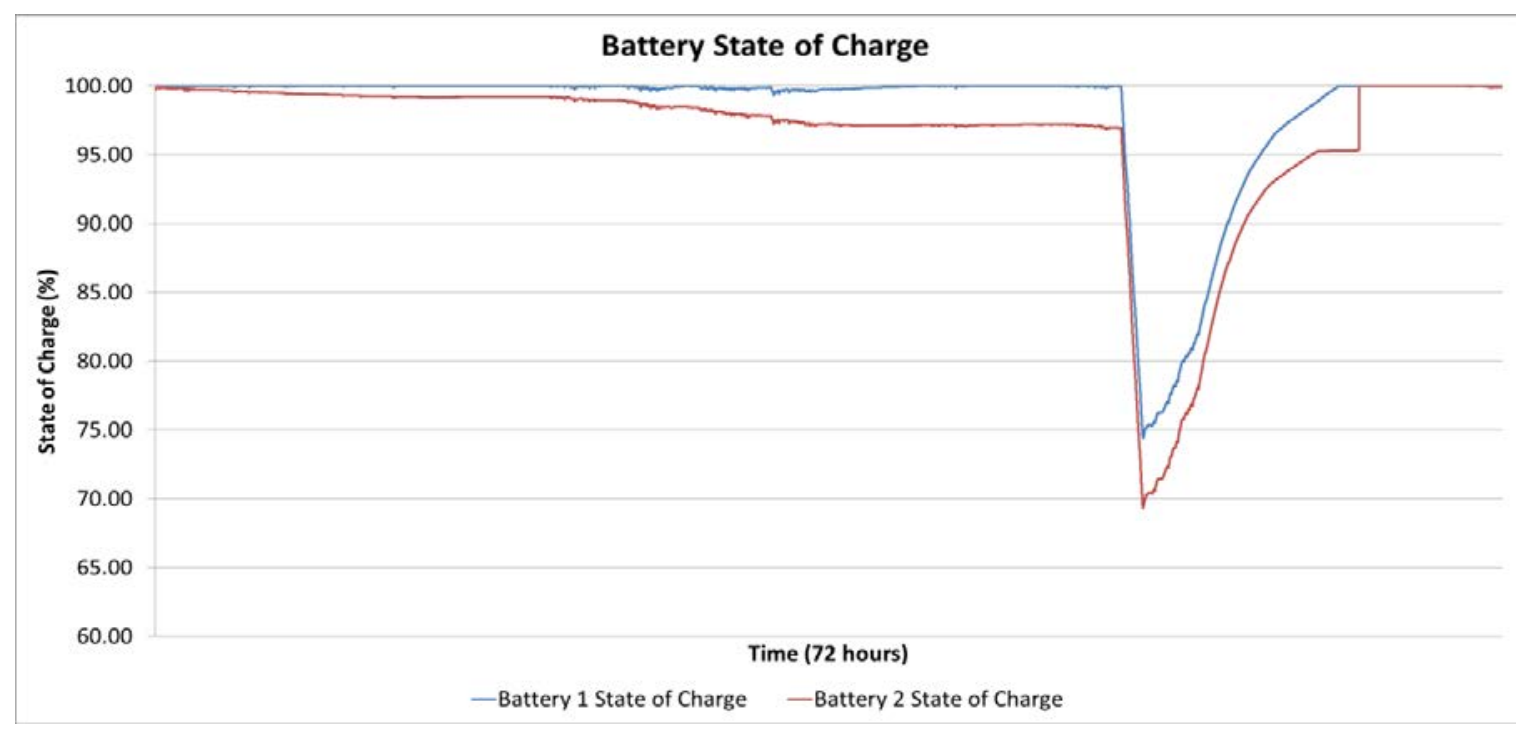

Figure E-37. Battery State of Charge over 72 Hours

\section{E.1.4.3 Photovoltaic Array Testing}

The photovoltaic array performed as expected and properly synchronized to the microgrid within minutes. Specific energy output for the 72-hour test is shown in Table E-4 and Figure E-38. October 18 and October 21 were partial days as testing started at 10:07 a.m. on October 18 and ended at 10:27 a.m. on October 21.

Table E-4. PV System Energy Output

\begin{tabular}{lccccc}
\hline & October 18, 2012 & October 19, 2012 & October 20, 2012 & October 21, 2012 & Total \\
\hline $\begin{array}{l}\text { Energy } \\
(\mathrm{kWh})\end{array}$ & 61.59 & 7.95 & 47.89 & 11.43 & 128.87 \\
\hline
\end{tabular}




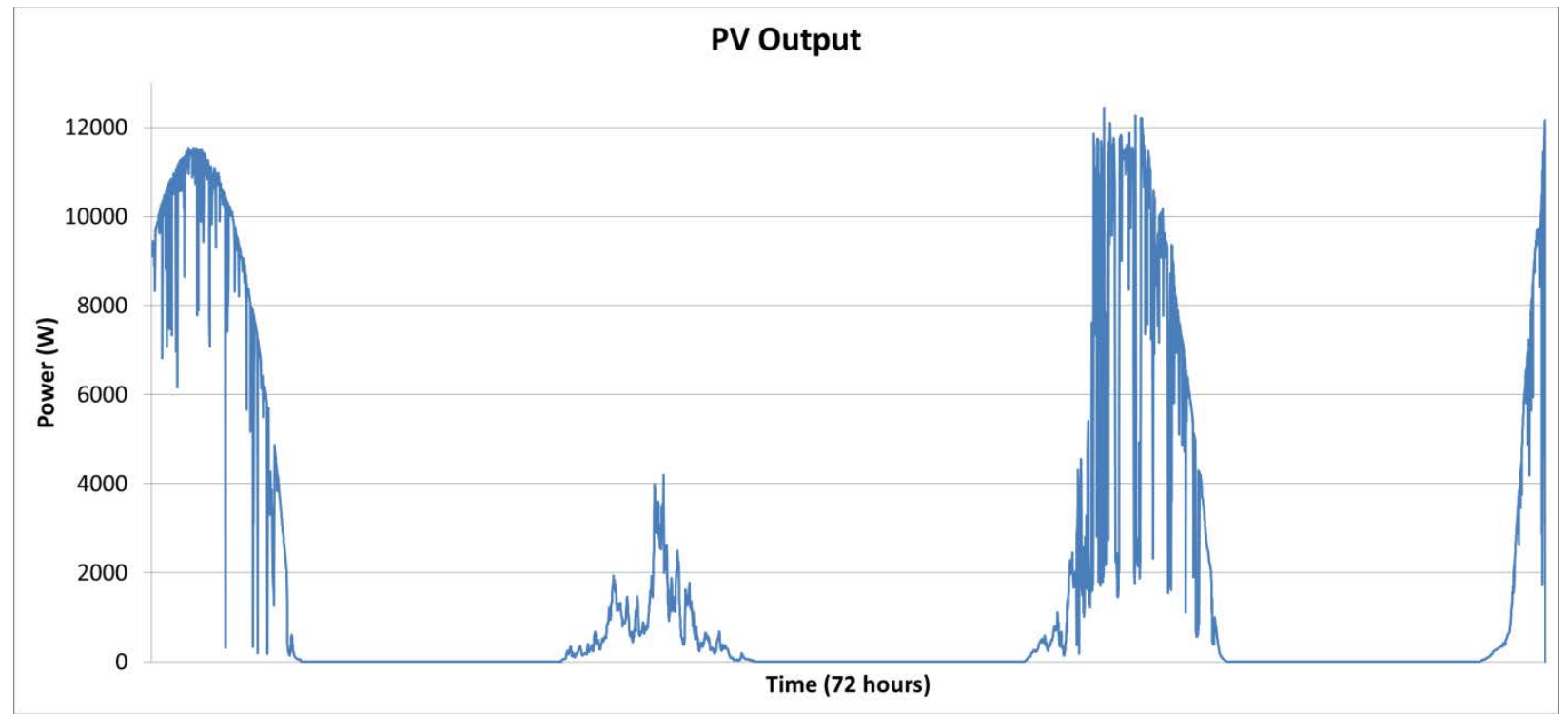

Figure E-38. PV System Power Output

\section{E.1.4.4 Energy Efficient Shelter and Tent Testing}

Daily and total energy for north camp tents and shelters are shown in Table E-5. Plug loads for some structures are shown in Table E-6 (only three tents were set up to measure plug loads). Daily and total energy for south camp tents are shown in Table E-7. The large energy variation between south camp and north camp tents $(-40.0 \%)$ is attributable to the non-baseline tent insulation liners in the south camp tents, the lower average temperature, and the lighter overall troop loading in the south camp. Temperature stability data of the shelters vs. north and south camp tents is shown in Table E-8.

Table E-5. Total Energy for North Camp Shelters and Tents

\begin{tabular}{lcccc}
\hline \multicolumn{1}{c}{ Structure } & $\begin{array}{c}\text { Day 1 Energy } \\
\text { (kWh) }\end{array}$ & $\begin{array}{c}\text { Day 2 Energy } \\
\text { (kWh) }\end{array}$ & $\begin{array}{c}\text { Day 3 Energy } \\
\text { (kWh) }\end{array}$ & $\begin{array}{c}\text { Total Energy } \\
\text { (kWh) }\end{array}$ \\
\hline Shelter 58 & 14.46 & 13.75 & 17.71 & 45.92 \\
Shelter 59 & 10.61 & 7.95 & 15.41 & 33.97 \\
Shelter 60 & 13.14 & 8.99 & 16.26 & 38.38 \\
Tent 25 & 179.74 & 106.70 & 199.56 & 486.01 \\
Tent 26 & 160.30 & 37.47 & 161.64 & 359.41 \\
Tent 27 & 157.29 & 97.23 & 161.61 & 416.13 \\
Tent 28 & 139.28 & 74.23 & 162.63 & 376.14 \\
Tent 29 & 83.45 & 45.98 & 125.72 & 255.13 \\
Tent 30 & 107.83 & 41.71 & 169.13 & 318.66 \\
Tent 31 & 88.56 & 47.08 & 122.83 & 258.47 \\
Tent 32 & 183.10 & 98.24 & 95.95 & 377.29 \\
Average Shelter & 12.74 & 10.23 & 16.46 & 39.43 \\
Average Tent & 137.44 & 68.58 & 149.88 & 355.91 \\
\% Savings & $90.7 \%$ & $85.1 \%$ & $89.0 \%$ & $88.9 \%$ \\
\hline
\end{tabular}


Table E-6. Plug Loads for Three Tents

\begin{tabular}{lcccc}
\hline \multicolumn{1}{c}{ Structure } & $\begin{array}{c}\text { Day 1 Energy } \\
(\mathbf{k W h})\end{array}$ & $\begin{array}{c}\text { Day 2 Energy } \\
\text { (kWh) }\end{array}$ & $\begin{array}{c}\text { Day 3 Energy } \\
\text { (kWh) }\end{array}$ & $\begin{array}{c}\text { Total Energy } \\
\text { (kWh) }\end{array}$ \\
\hline Tent 30 & 3.47 & 5.13 & 4.43 & 13.03 \\
Tent 31 & 6.45 & 5.29 & 4.56 & 16.30 \\
Tent 32 & 7.36 & 7.46 & 7.73 & 22.55 \\
Average plug load & 5.76 & 5.96 & 5.57 & 17.29 \\
\hline
\end{tabular}

Table E-7. Total Energy for South Camp Tent

\begin{tabular}{lcccc}
\hline \multicolumn{1}{c}{ Structure } & $\begin{array}{c}\text { Day 1 Energy } \\
\text { (kWh) }\end{array}$ & $\begin{array}{c}\text { Day 2 Energy } \\
\text { (kWh) }\end{array}$ & $\begin{array}{c}\text { Day 3 Energy } \\
\text { (kWh) }\end{array}$ & $\begin{array}{c}\text { Total Energy } \\
\text { (kWh) }\end{array}$ \\
\hline Tent 1 & 77.43 & 40.39 & 155.44 & 273.26 \\
Tent 2 & 124.52 & 50.46 & 115.74 & 290.71 \\
Tent 3 & 42.14 & 51.78 & 69.04 & 162.97 \\
Tent 4 & 48.03 & 40.02 & 73.20 & 161.25 \\
Tent 5 & 44.11 & 40.41 & 72.84 & 157.35 \\
Tent 6 & 84.03 & 39.68 & 99.65 & 223.35 \\
Tent 7 & 60.54 & 54.55 & 99.20 & 214.28 \\
Tent 8 & 66.41 & 55.78 & 103.12 & 225.31 \\
Average south camp & 68.40 & 46.63 & 98.53 & 213.56 \\
tent & & & & \\
\% difference from & $-50.2 \%$ & $-32.0 \%$ & $-34.3 \%$ & $-40.0 \%$ \\
average north camp & & & & \\
tent & & & & \\
\hline
\end{tabular}

Table E-8. Temperature Stability

\begin{tabular}{lccc}
\hline \multicolumn{1}{c}{ Structure } & $\begin{array}{c}\text { Average Temperature } \\
\left({ }^{\circ} \mathrm{F}\right)\end{array}$ & Spread & Standard Deviation \\
\hline Shelter 58 & SAGE Shelters & & \\
Shelter 59 & 72.05 & 18.36 & 2.91 \\
Shelter 60 & 70.45 & 17.10 & 3.29 \\
Shelter Average & 72.55 & 13.46 & 1.83 \\
\hline \multicolumn{4}{c}{ North Camp Tents } \\
\hline Tent 25 & 71.65 & 16.31 & 2.68 \\
Tent 26 & 74.24 & 24.66 & \\
Tent 27 & 73.26 & 25.97 & 4.72 \\
Tent 28 & 74.86 & 37.24 & 5.74 \\
Tent 29 & 74.59 & 26.84 & 7.39 \\
Tent 30 & 70.32 & 27.95 & 4.94 \\
Tent 31 & 72.01 & 29.03 & 5.03 \\
Tent 32 & 73.76 & 26.62 & 4.88 \\
North camp tent average & 74.71 & 44.19 & 5.38 \\
\hline
\end{tabular}




\begin{tabular}{|c|c|c|c|}
\hline \multicolumn{4}{|c|}{ South Camp Tents } \\
\hline Tent 1 & 70.22 & 36.68 & 7.60 \\
\hline Tent 2 & 73.94 & 26.06 & 4.42 \\
\hline Tent 3 & 67.63 & 30.44 & 7.28 \\
\hline Tent 4 & 68.26 & 25.25 & 5.40 \\
\hline Tent 5 & 68.49 & 29.59 & 6.57 \\
\hline Tent 6 & 69.50 & 23.20 & 4.90 \\
\hline Tent 7 & 68.33 & 31.10 & 7.21 \\
\hline Tent 8 & 69.00 & 20.66 & 4.93 \\
\hline South camp tent average & 69.42 & 27.87 & 6.04 \\
\hline
\end{tabular}

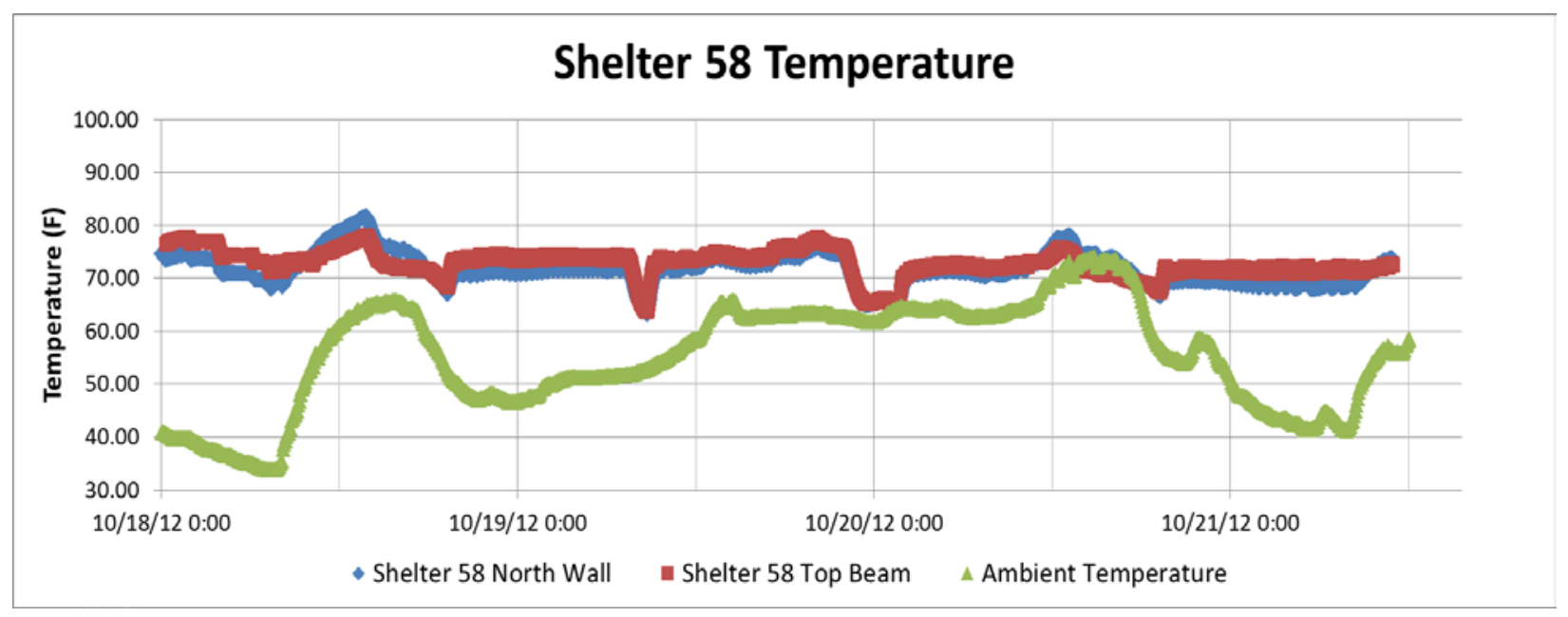

Figure E-39. Shelter 58 Temperatures

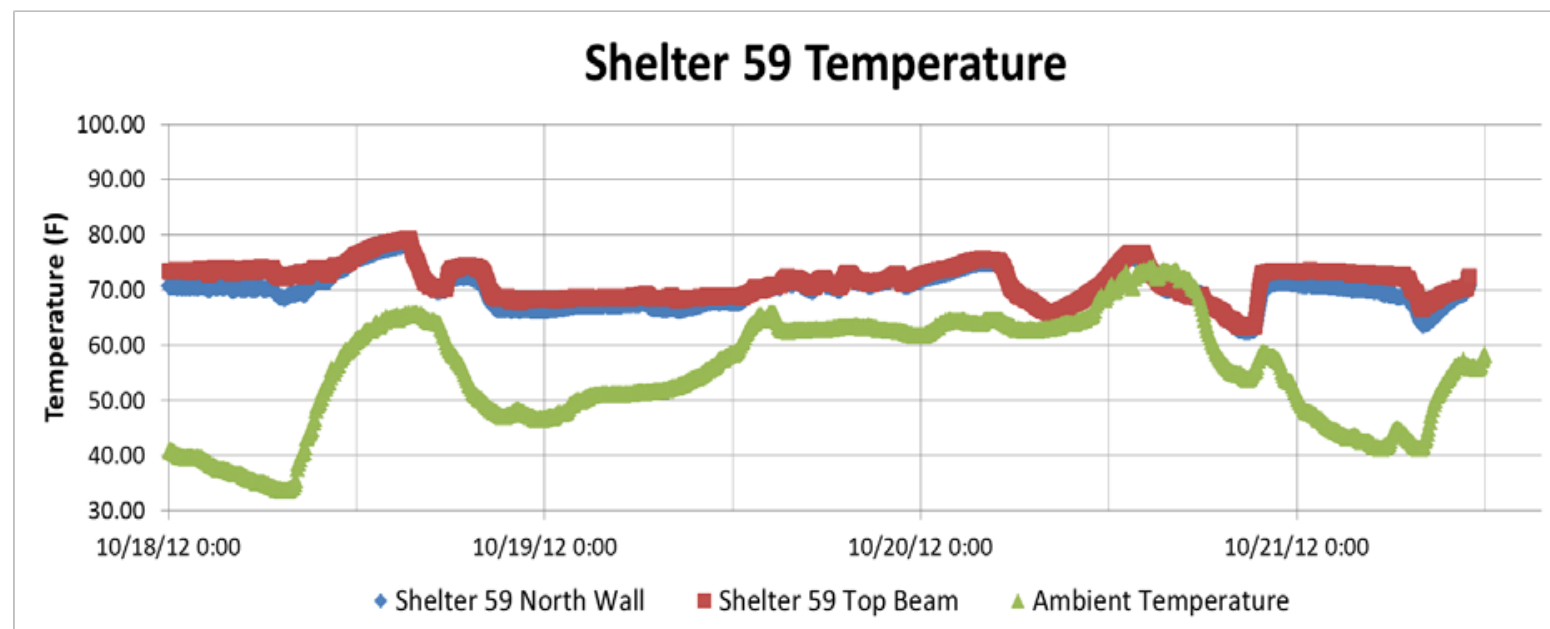

Figure E-40. Shelter 59 Temperatures 


\section{Shelter 60 Temperatures}

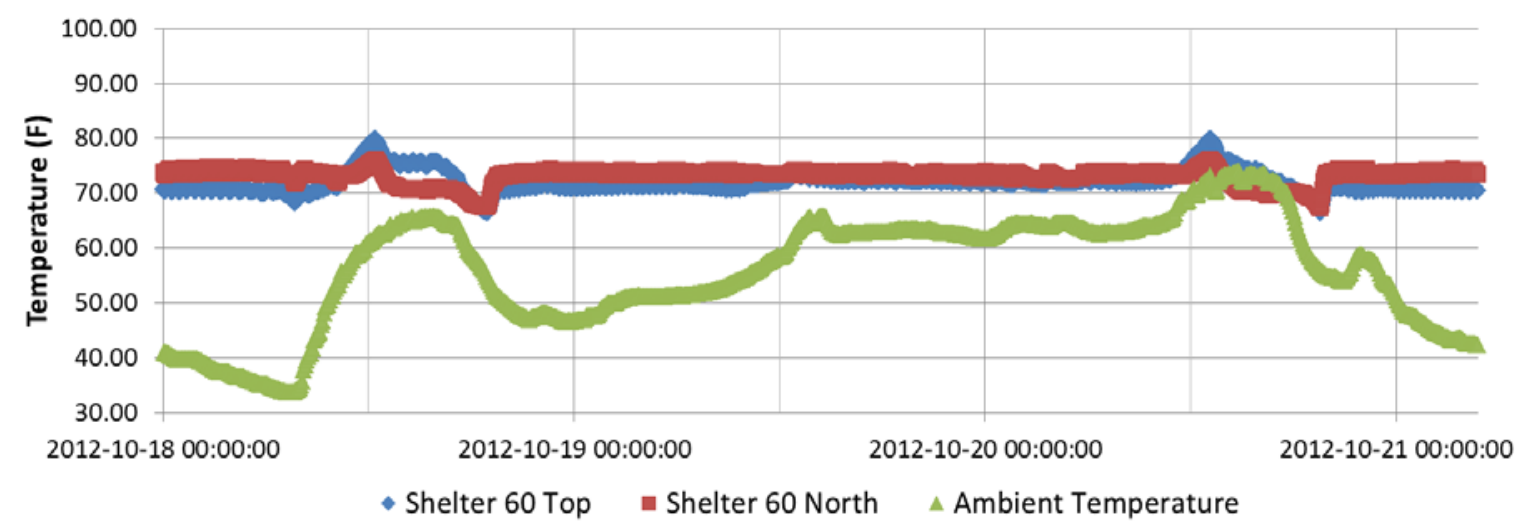

Figure E-41. Shelter 60 Temperatures

\section{Tent 25 Temperatures}

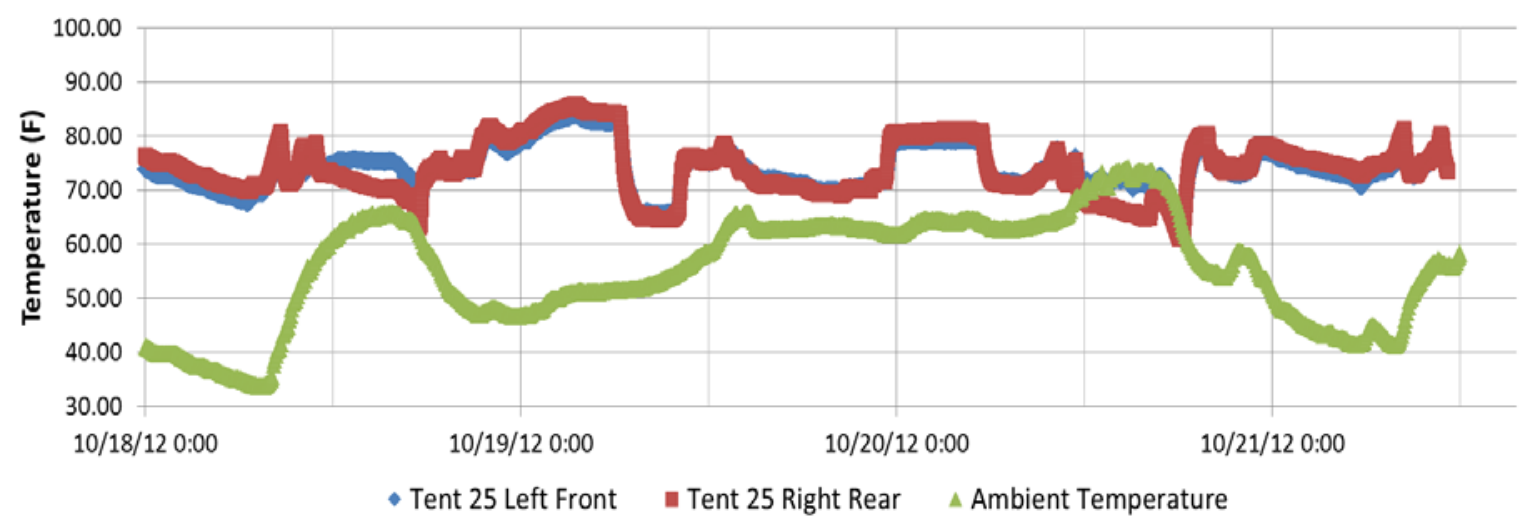

Figure E-42. Tent 25 Temperatures (North Camp)

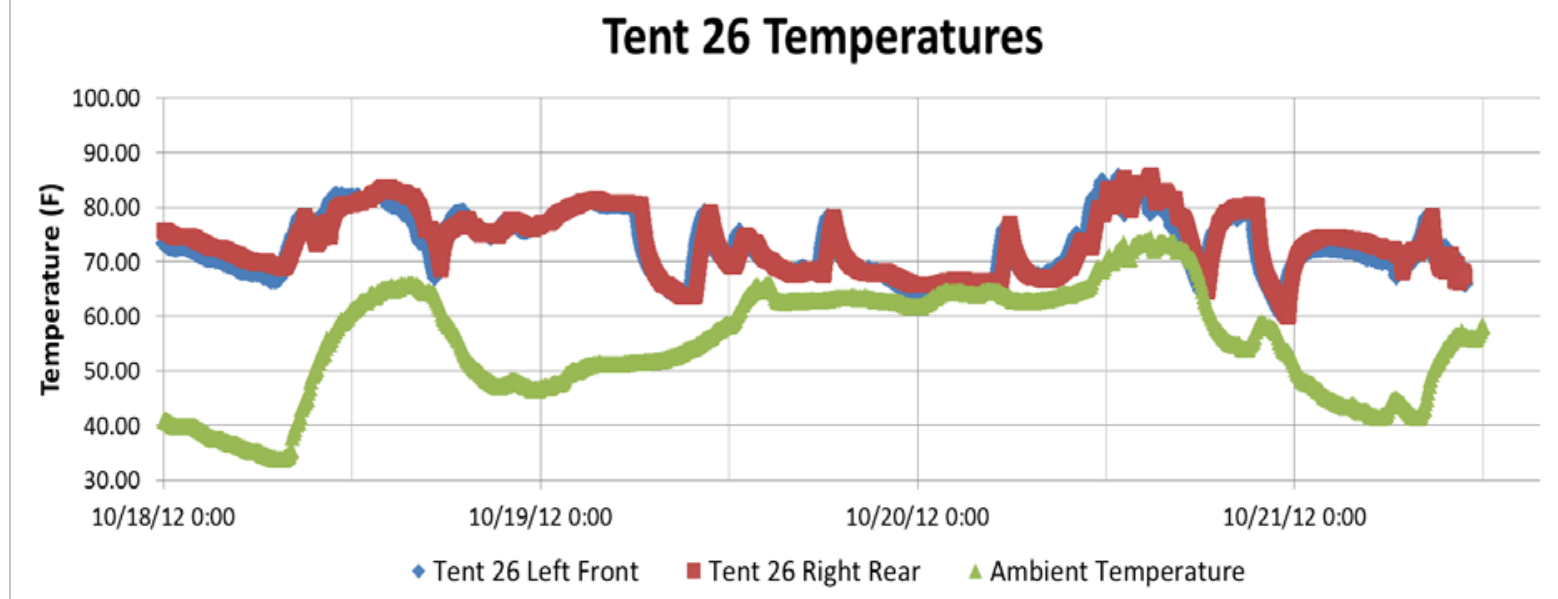

Figure E-43. Tent 26 Temperatures (North Camp) 


\section{Tent 27 Temperature}

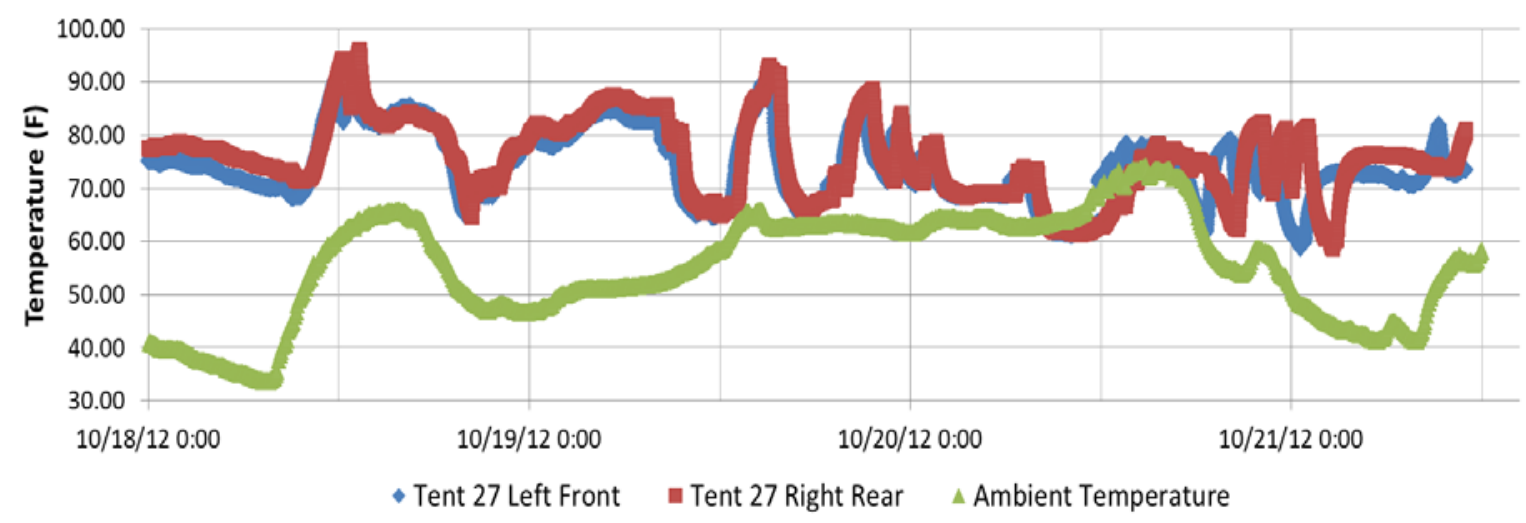

Figure E-44. Tent 27 Temperatures (North Camp)

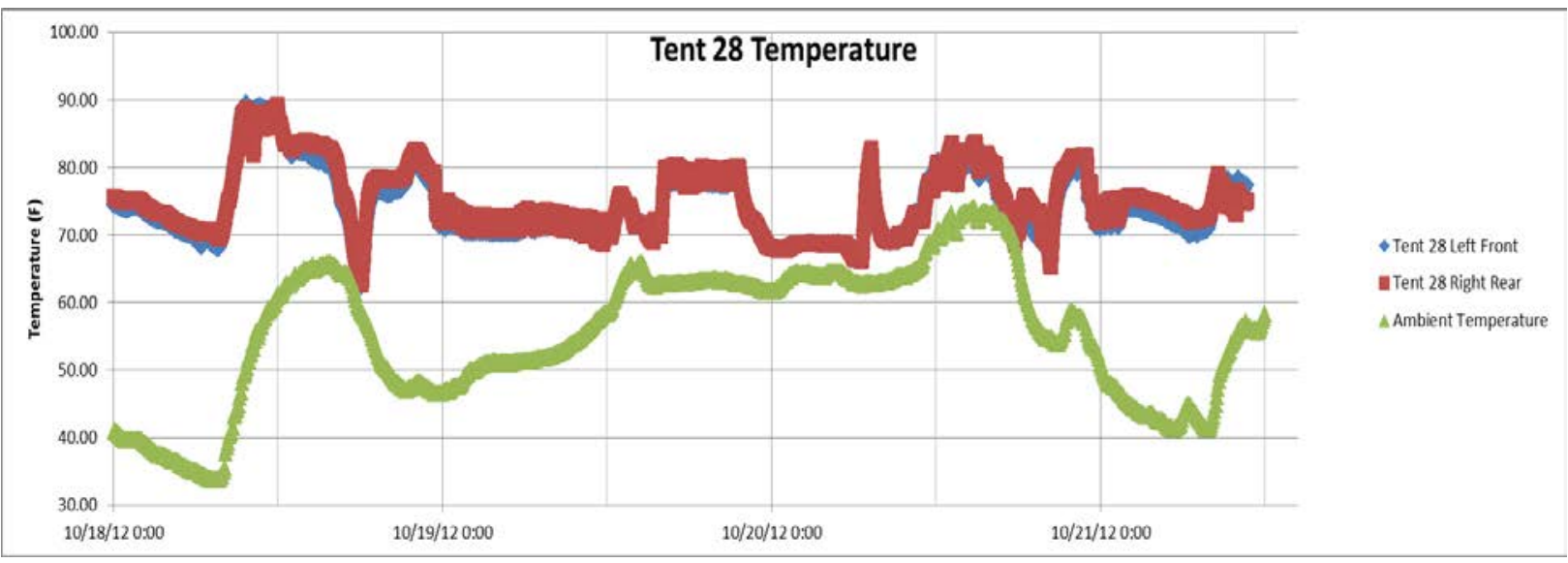

Figure E-45. Tent 28 Temperatures (North Camp)

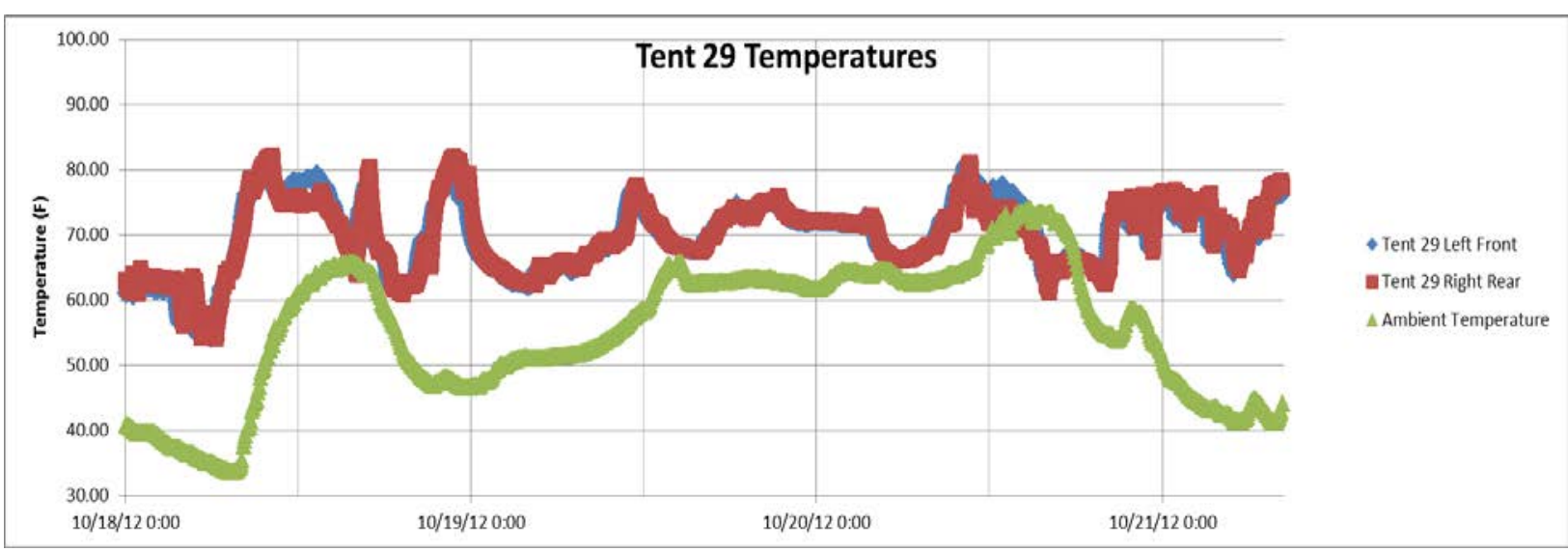

Figure E-46. Tent 29 Temperatures (North Camp) 


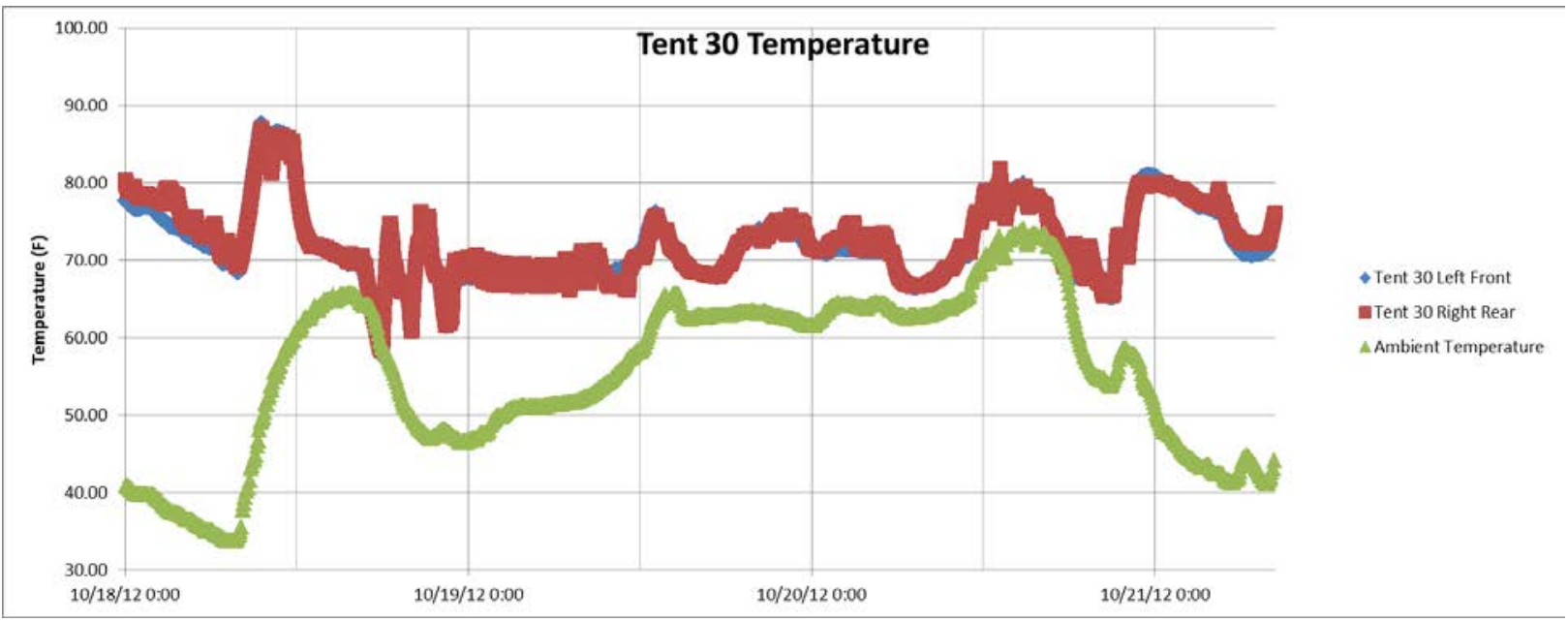

Figure E-47. Tent 30 Temperatures (North Camp)

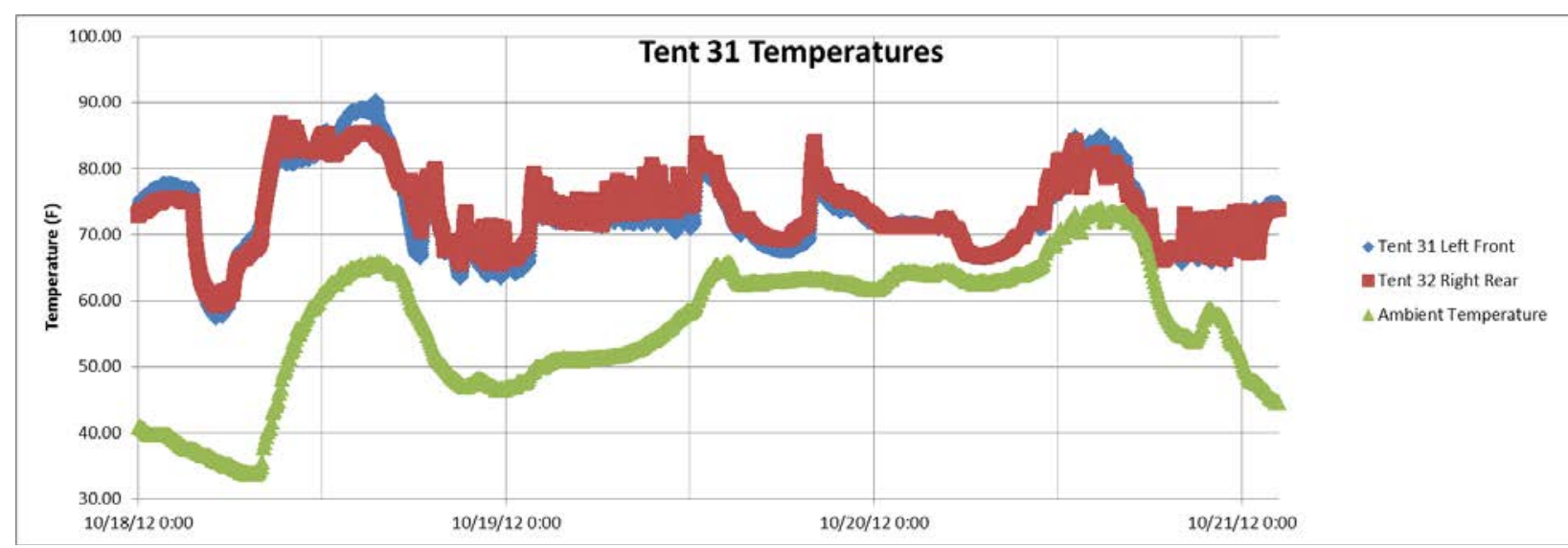

Figure E-48. Tent 31 Temperatures (North Camp)

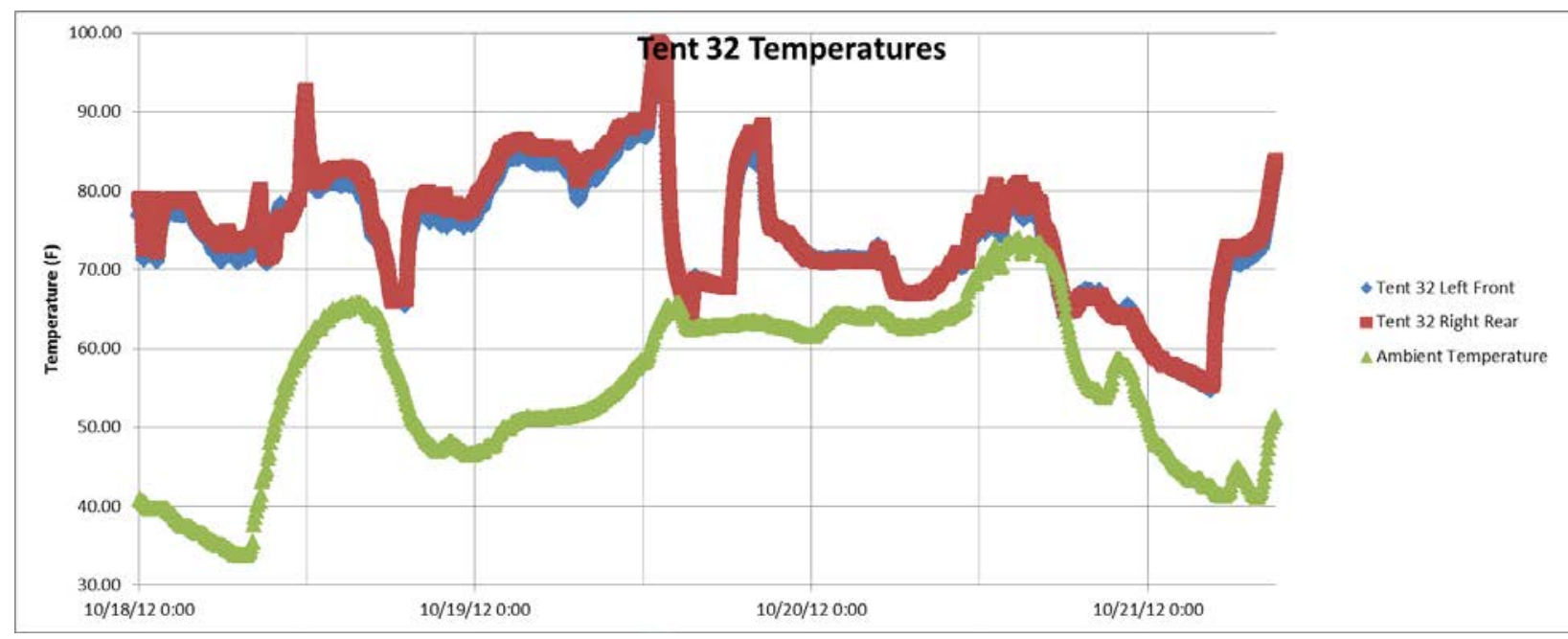

Figure E-49. Tent 32 Temperatures (North Camp) 


\section{E.1.4.5 Solar Hot Water Heating System Testing}

The SAGE SHW tests were performed in phases 2 and 3. Phase 2 included one day controlled simulated tests performed on June 20, 2012 and October 11, 2012, and Phase 3 was performed October 18, 2012 through October 21, 2012, and included three continuous test days with troops occupying the camps.

The solar hot water (SHW) system analysis involved calculating an energy balance (input and outputs) over time, across an applied control volume (dotted line in Figure E-50) including:

\section{Inputs:}

- The electrical energy input to the pumps, controller, lights, etc. $\left(\mathrm{Q}_{\mathrm{E}}\right)$; measured and evaluated over specified time period, (kWh).

- The solar energy input to the collectors $\left(\mathrm{Q}_{\mathrm{I}}\right)$; measured horizontally $\left(\mathrm{W} / \mathrm{m}^{2}\right)$, and evaluated the applied area of panels $\left(\mathrm{m}^{2}\right)$ over specified time period, $(\mathrm{kWh}){ }^{23}$

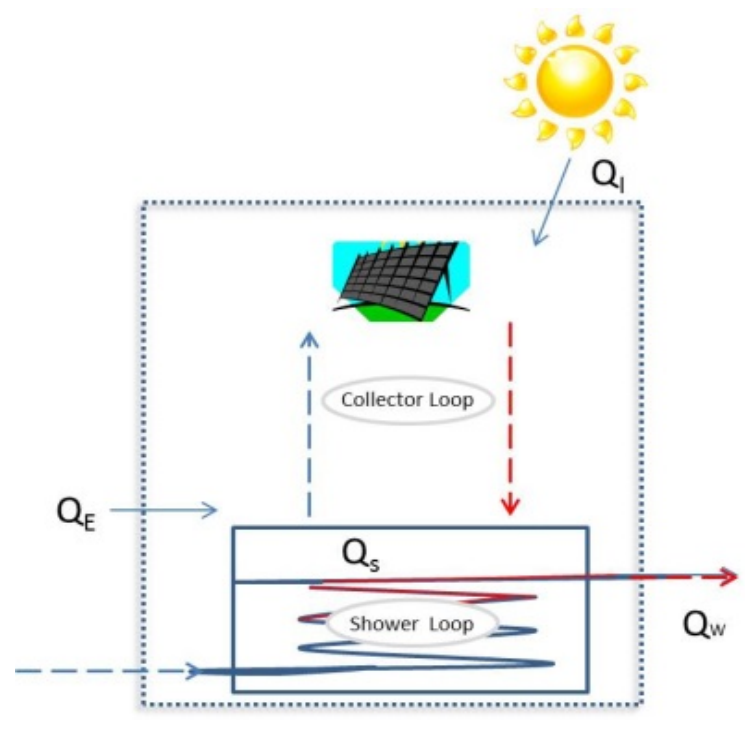

Figure E-50. Control Volumes for Analysis

\section{Outputs:}

- The hot thermal water output (available energy) to the showers $\left(\mathrm{Q}_{\mathrm{w}}\right)$; was determined by the following equations:

$$
Q_{w}=c \cdot M \cdot\left(T_{h}-T_{c}\right)
$$

where $\quad c=1.007 \frac{B T U}{l b \cdot{ }^{\circ} \mathrm{F}}=$ the specific heat of water, ${ }^{24}$ at $100^{\circ} \mathrm{F}=0.998$

$$
\begin{aligned}
T_{c}= & \text { temperature of cold water supplied to } \mathrm{SHW}\left({ }^{\circ} \mathrm{F}\right) ; \text { measured } \\
T_{h}= & \text { temperature of water exiting } \mathrm{SHW} \text { hot water system }\left({ }^{\circ} \mathrm{F}\right) ; \text { measured } \\
M_{W}= & \text { mass }(\mathrm{lbm}) \text { of water through the } \mathrm{SHW} \text { heat exchanger, during the } \\
& \text { specified time period; measured. }
\end{aligned}
$$

Although the storage tank energy (Qs) was within the control volume, it was also accounted for in the analysis. The two energy transfer loops, the collector and shower loops operate independently of each other. The collector loop operates (e.g., is "active"), when solar energy is available, and preset control conditions are met, activating the system pumps to lift the heat

\footnotetext{
${ }^{23}$ Evaluated when system was active (e.g., pumps running).

24 "c" is the specific heat $\left(\mathrm{Btu} / \mathrm{lbm}{ }^{-} \mathrm{F}\right)$ dependent on the temperature of water or mean temp of range .
} 
transfer fluid (e.g., SAGE SHW system used potable water), up to the collectors to be heated. Subsequently, the hot water is drained back by gravity into the storage tank.

The following equation was used to calculate the energy stored in the tank:

$$
Q_{S}=M\left(H_{2}-H_{1}\right)
$$

where

$$
\begin{aligned}
\mathrm{H}_{1}(\mathrm{Btu} / \mathrm{lbm})= & \text { enthalpy of the collector heat transfer fluid evaluated at } \mathrm{T}_{1},\left({ }^{\circ} \mathrm{F},\right. \\
& \text { temperature of the water in the storage tank at the beginning of the test }) \\
\mathrm{H}_{2}(\mathrm{Btu} / \mathrm{lbm})= & \begin{aligned}
\text { enthalpy of the collector heat transfer fluid evaluated at } \mathrm{T}_{2},\left({ }^{\circ} \mathrm{F},\right. \\
\text { temperature of water in the storage tank at the end of the test) }
\end{aligned} \\
\mathrm{M}= & \text { mass of water in tank (415 gallons converted at average temperature). }
\end{aligned}
$$

The shower loop only operates when shower water is demanded; thus depending on the time of day, the collector loop may be the only "active" loop in the system, heating the water in the storage tank. Given a demand for shower water, the shower water loop is activated and cold potable water is supplied to the system. The incoming water is sent through the heat exchanger piping submerged inside the tank to be preheated and sent to the showers via the auxiliary fuel fired heater.

The auxiliary fuel fired heater is downstream of the SHW system, and it adds heat, as necessary, to the preheated shower water before entering the showers to meet the desired temperature. The auxiliary fuel-fired water heater is not part of the energy balance across the SHW system calculation since it was outside of the control volume applied to the system. Although the fuel consumed by the AWH 400 heaters, water flow and temperature measurements in and out of the AWH 400 heaters were measured which enabled calculating an energy balance across these units.

The SAGE SHW system energy output calculated for each test are shown the Table E-9.

Table E-9. SAGE SHW System Energy Output Calculations by Test

\begin{tabular}{lc}
\hline \multicolumn{1}{c}{ Test Date/Type } & $\begin{array}{c}\text { SHW System Energy } \\
\text { Output (Btu/day) }\end{array}$ \\
\hline June 2012/Simulation-Phase 2 & 367,073 \\
October 11, 2012/Simulation-Phase 2 & 315,982 \\
October 18, 2013/Occupancy-Phase 3 & 158,171 \\
October 19, 2013/Occupancy-Phase 3 & 35,586 \\
October 20, 2013/Occupancy-Phase 3 & 126,989 \\
\hline
\end{tabular}

In addition to performing energy balance calculations, the system was characterized according to some common parameters. One common parameter that describes a domestic hot water system is the solar fraction (SF). The SF, as defined by ASHRAE 95-1987, ${ }^{25}$ is the

\footnotetext{
${ }^{25}$ Methods of testing to determine the thermal performance of solar domestic water heating systems (ASHRAE 95$1987)$.
} 
fraction of the total hot water load supplied by solar energy. The higher the solar fraction, the greater the solar contribution to water heating, which reduces the energy required by the backup water heating. The solar fraction varies from 0 to 1.0 , and is highly dependent on geographic location; the prototype SAGE system was tested in Massachusetts which per Figure E-51 typically delivers solar fractions around $50 \%$ range. $^{26}$ However, a study performed on a Massachusetts home with a similar system (a solar hot water indirect closed loop system with flat plate collectors) showed an average $61 \%$ solar fraction after annualizing one year's data. ${ }^{27}$ It also revealed a broad range over the year; a solar fraction of $28 \%$ solar fraction in December and $87 \%$ in August.

Likewise, our testing resulted in a wide range of solar fractions as the system was exposed to diverse demand loads and weather conditions. During the phase 2 test days, the sun was always shining and the consumption peaked in the morning and evening-allowing the storage to recuperate throughout the day. However, during the phase 3 test days, the demand was more diverse and the second test day, October 19, was mostly cloudy. Also impacting the results was the fact that before the start of the phase 2 simulation tests and the first day in phase 3, the SAGE SHW system had hot water $\left(>110^{\circ} \mathrm{F}\right)$ already in the storage tank. The solar fractions (as defined by ASHRAE 95-1987) for both test phases are shown in Table E-10.

\footnotetext{
${ }^{26}$ Assumes a constant load profile for a single family home and electric auxiliary water heater. Downloaded 1/14/14 at http://en.openei.org/w/index.php?title=File:NREL-TMY3-Solar-Factor.pdf; published as: Cassard, Hannah; Denholm, Paul; and Ong, Sean; "Break-even Cost for Residential Solar Water Heating in the United States: Key Drivers and Sensitivities,” Technical Report NREL/TP-6A20-48986; February 2011.

27 Aldrich, Robb and Vijayakumar, Gayathri; "Cost, design and performance of solar hot water in cold-climate homes”, Steven Winter Associates, Inc., 50 Washington St Norwalk, CT 06854
} 


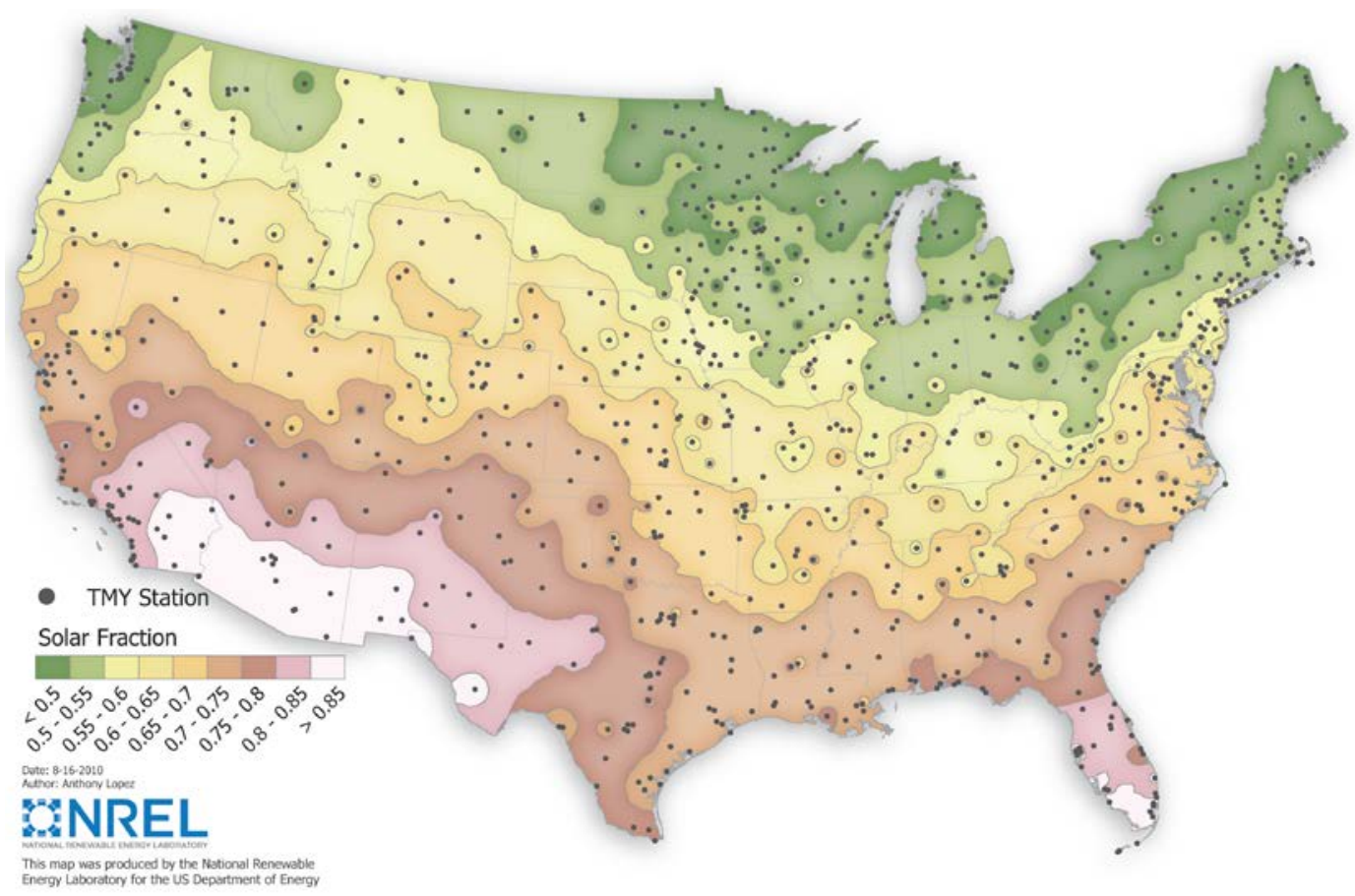

Figure E-51. Solar Fraction - The Percent of Typical Annual Residential Water Heating Needs (Source: NREL)

Table E-10. Phase 2 and 3 Solar Fractions

\begin{tabular}{lc}
\hline \multicolumn{1}{c}{ Test Date/Type } & Solar Fraction \% \\
\hline June 2012/Simulation-Phase 2 & 61 \\
October 11, 2012/Simulation-Phase 2 & 53 \\
October 18, 2013/Occupancy-Phase 3 & 58 \\
October 19, 2013/Occupancy-Phase 3 & 10 \\
October 20, 2013/Occupancy-Phase 3 & 14 \\
\hline
\end{tabular}

The SAGE system utilized the Morning Star Collector (Model MSC-32). The MSC-32 collector was tested by two independent organizations, the Solar Rating Certification Corporation (SRCC) and the Florida Solar Energy Center (FSEC). These organizations perform highly controlled tests based on ASHRAE standards ${ }^{28}$ and compute efficiency equations, and performance ratings on a per collector panel basis. After graphing and comparing the solar panel ratings of both organizations against the SAGE system test data, the SRCC performance ratings were used for the analysis because the SRCC performance curves manifested a better correlation to our test data (Figure E-52, SRCC Certification sheet for the MSC-32 solar collector).

\footnotetext{
${ }^{28}$ SRCC tests for glazed flat-plate collectors are based on the American Society of Heating, Refrigerating, and Air Conditioning Engineers (ASHRAE) Standard 93-1986; Methods of Testing to Determine the Thermal Performance of Solar Collectors.
} 


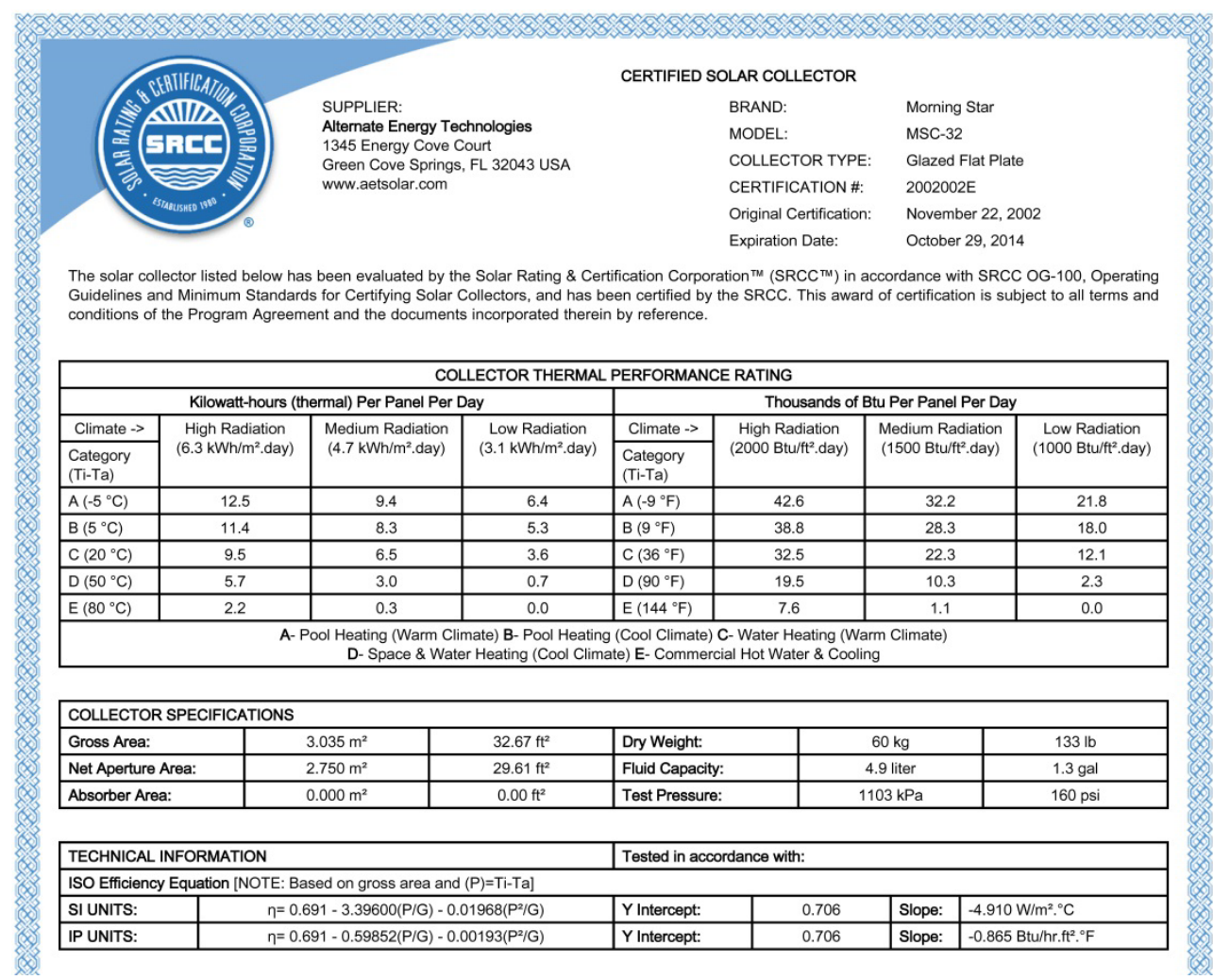

Figure E-52. SRCC Certification Sheet for MSC-32 Panels

The SRCC collector thermal performance ratings (energy output) are provided for three categories; high, medium and low levels of solar radiation ${ }^{29}$ (energy input in Btu/square feet per day) and as a function of the following variables:

- the ambient temperature $(\mathrm{Ta}),{ }^{\circ} \mathrm{F}$

- the inlet temperature to the collector $(\mathrm{Ti}),{ }^{\circ} \mathrm{F}$.

The SRCC ratings define the relationships, generate algorithms and depict curves for these key variables that impact performance of solar collectors. The generated algorithms from these tests defined the key variables that impact performance of solar collectors and in turn (albeit not comprehensively) the performance of the system. ${ }^{30}$

To reveal the existing discrepancies between the performance of solar collectors and total system, the SAGE SHW data was converted to a per panel basis (as required), and the algorithms were calculated using the SAGE SHW system field test data such as:

- average inlet collector temperature ( $\mathrm{Ti}=$ storage temperature) and average ambient temperature (Ta); evaluated when systems are "active"

\footnotetext{
${ }^{29}$ Solar Irradiance (aka insolation) - the "quantity of solar radiation incident on unit surface area in unit time."; as defined in American Society of Heating, Refrigerating, and Air Conditioning Engineers (ASHRAE) Standard 93-1986; Methods of Testing to Determine the Thermal Performance of Solar Collectors.

${ }^{30}$ This assumes that the collector efficiency parameters are fixed for a given collector model.
} 
- energy output of the SHW system and energy input of the SHW system to calculate efficiency; the energy input was calculated two ways, with solar energy input only and with a combined solar energy input, energy storage and parasitic power.

Subsequently, the SRCC MSC-32 collector panel performance curves for all three categories; high, medium and low levels of solar radiation curves were graphed and evaluated against the SAGE system performance data and the analysis focused on the medium radiation day which provided the closest correlation to our test data (Figure E-53).

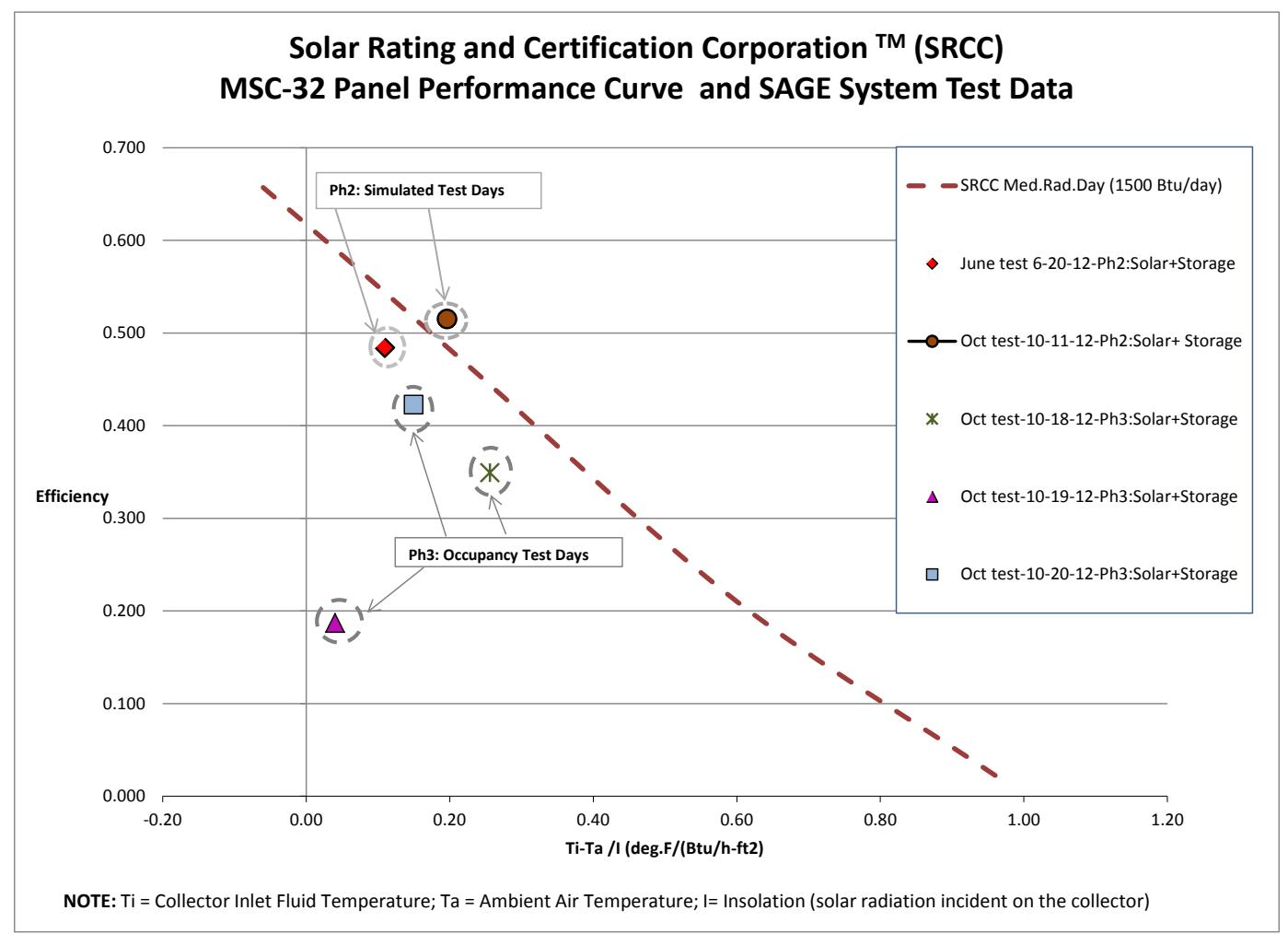

Figure E-53. SRCC Panel Performance Curve versus Sage SHW System Test Results

Figure E-53 depicts that on a medium radiation day, the maximum efficiency of the MSC-32 solar collector panel $\{$ when $\mathrm{Ti}-\mathrm{Ta}=0$, when the average collector inlet fluid temperature $(\mathrm{Ti})$ entering the collector is the same temperature as the average ambient temperature (Ta) $\}$ is slightly greater than $60 \%$. The average efficiency for the SAGE phase 2 simulation tests at the average temperatures and insolation for the test days was 50\%. However, the average efficiency for the SAGE phase 3 occupancy test (at the average temperatures and insolation for the test days) was $32 \%$, and ranged from $42 \%$ to $19 \%$.

It was expected that the phase 2 efficiencies would be better than phase 3 because the phase 2 tests were simulated (e.g., controlled) and performed during optimal conditions with a preheated storage tank while the occupancy tests were uncontrolled with actual conditions. During the phase 3 occupancy test, the SHW system was exposed to diverse demand loads shortening and/or eliminating recuperation periods and subject to unfavorable weather conditions, such as the extremely cloudy day creating the outlier on October 19, 2012 with the lowest efficiency of $19 \%$. 
Although the graph indicates predicted results, there is still a discrepancy associated with the fact that the SRCC panel performance curves are based only on the collector; thus the losses associated with the system are not accounted for in the SRCC performance algorithms. Therefore, to generate a curve depicting a system performance versus the same parameters (Ti-Ta/I), a new set of performance curves were derived applying the following system losses (totaling up to 10\%) to the SRCC solar panel performance curves:

- Standby losses associated with the storage tank based on the manufacturer data averaged of several insulated storage tanks and spot checks of measured field data shows a typical range of $1 / 4$ to $1 / 2{ }^{\circ} \mathrm{F}$ per hour; which resulted in a $\sim 2 \%$ loss.

- Distribution losses; resulting in an average $3 \%{ }^{31}$ loss.

- Submersible heat exchanger efficiency losses are in the range of $~ 90-95 \% ;{ }^{32}$ thus, a $5 \%$ loss was applied.

As Figure E-54 depicts, on a medium radiation day the maximum efficiency of the derived SAGE SHW system performance curve (when $\mathrm{Ti}-\mathrm{Ta}=0$ ), is approximately $55 \%$. Further, the graph shows that with the exception of the outlier, there is a fairly close relationship between the derived system curve and the SAGE SHW test results.

However, relying on performance ratings from the SRCC) (or the FSEC) with ratings generated under controlled conditions with arguably inaccurate results, is not ideal. Further, while the SAGE field tests, successfully demonstrated the "proof of concept" the tests were subject to constraints that resulted in moderate data collection and although system losses were observed and tracked periodically during the testing, it was not the focus of the study therefore; the losses used to derive the system performance curve could not be validated. As a result, the

\footnotetext{
${ }^{31}$ http://www.fsec.ucf.edu/en/publications/pdf/FSEC-PF-429-07.pdf

${ }^{32}$ Lane, Tom; Solar Hot Water Systems, Lessons learned 1977 to Today; Energy Conservation Services, Florida, 2004; page 36.
} 
field tests results were used to calibrate a computer model, created in EnergyPlus. Further, the analysis performed was utilized to corroborate the model results which indicated a conservative estimate to global savings.

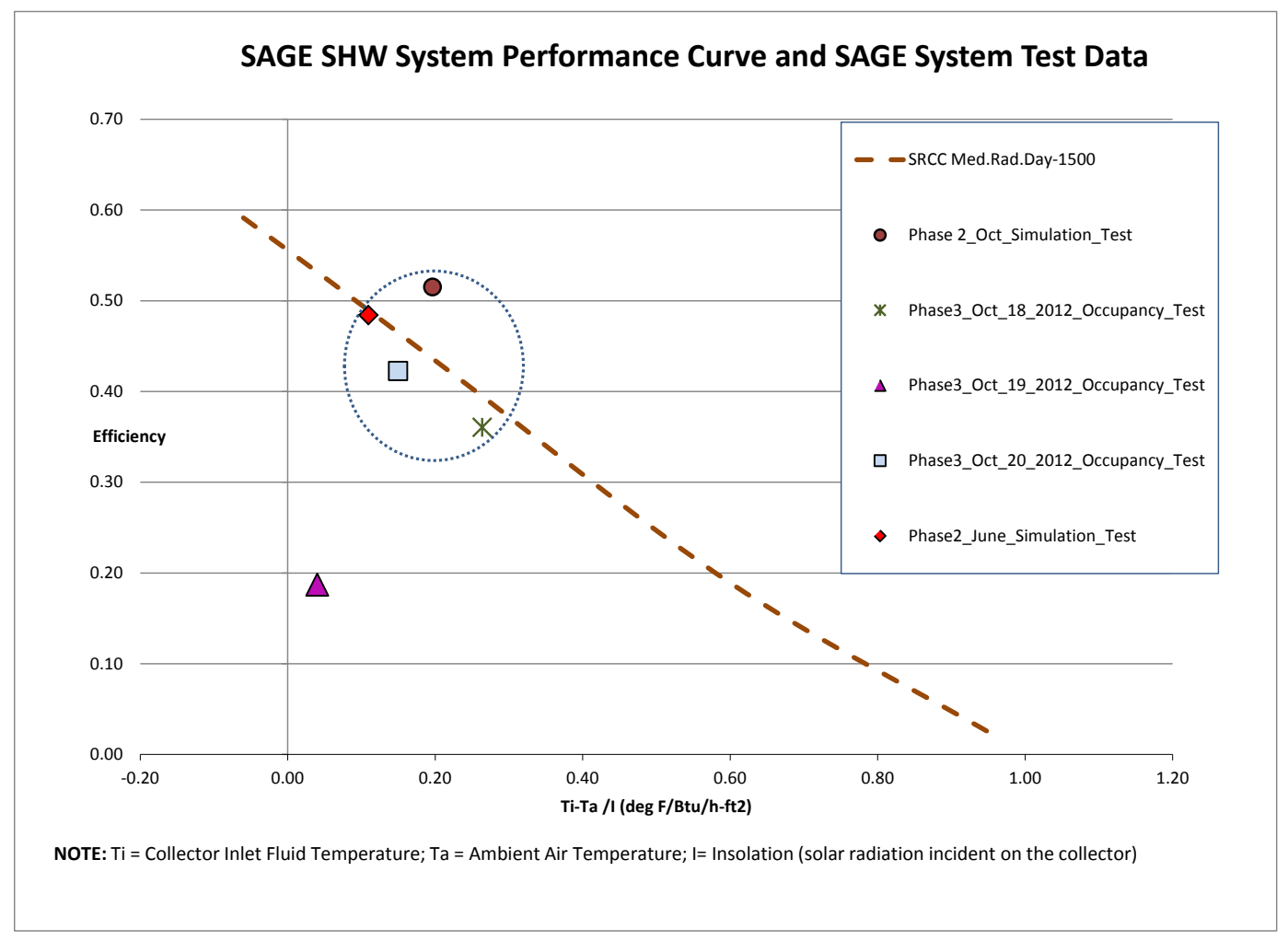

Figure E-54. SAGE SHW System Efficiency Curve 



\section{Appendix $F$ Acronyms and Abbreviations}

${ }^{\circ} \mathrm{C}$

${ }^{\circ} \mathrm{F}$

$\mathrm{AC}$

ACH50

AET

AFCS

AWH

BACnet

BCIL

BDI

BOM

Btu

CAN

CB-EMS

$\mathrm{CD}$

cfm

CFM50

$\mathrm{CON}$

COP

COTS

CSV

DC

DOD

DOE

DSOM

DX

ECU

EMC

EUI

FOB

FSEC

$\mathrm{ft}$

$\mathrm{ft}^{2}$

gal

gpm

GUI degree(s) Celsius

degree(s) Fahrenheit

alternating current

air changes per hour under 50 pascals of pressure

Alternate Energy Technologies, LLC

Army Facilities Components System

Army Water Heater

Building Automation and Control Network

Base Camp Integration Laboratory

bidirectional inverter

Bill of Materials

British thermal units

Controller Area Network

Contingency Base Energy Management System

compact disk

cubic foot/feet per minute

cubic feet per minute at 50 pascals

Certificate of Networthiness

coefficient of performance

commercial off-the-shelf

comma-separated value

direct current

U.S. Department of Defense

U.S. Department of Energy

Decision Support for Operations and Maintenance

direct expansion

environmental control unit

electromagnetic compatibility

energy use intensity

forward operating base

Florida Solar Energy Center

foot/feet

square foot/feet

gallons

gallon(s) per minute

graphical user interface 


\begin{tabular}{|c|c|}
\hline HMI & human-to-machine interface \\
\hline $\mathrm{hr}$ & hour(s) \\
\hline HSPF & Heating Seasonal Performance Factor \\
\hline HVAC & heating, ventilating, and air conditioning \\
\hline HWLSLD & Hard Wired Load Shed Load Demand \\
\hline $\mathrm{Hz}$ & hertz, cycles per second \\
\hline IEC & International Electrotechnical Commission \\
\hline IEEE & Institute of Electrical and Electronics Engineers \\
\hline IP & Internet Protocol \\
\hline ISO & International Organization for Standardization \\
\hline JCMS & Joint Construction Management System \\
\hline $\mathrm{kVA}$ & kilovolt ampere, apparent power \\
\hline $\mathrm{kVAr}$ & kilovolt amperes reactive, reactive power \\
\hline $\mathrm{kW}$ & kilowatts, unit of power \\
\hline $\mathrm{kWh}$ & kilowatt-hours, unit of energy \\
\hline $\mathrm{lbm}$ & pounds mass \\
\hline LED & light-emitting diode \\
\hline LIA & U.S. Army Logistics Innovation Agency \\
\hline LOGCAP & Logistics Civil Augmentation Program \\
\hline Mbps & megabits per second \\
\hline microgrid & $\begin{array}{l}\text { small-scale electrical distribution and transmission grid with distributed } \\
\text { generation }\end{array}$ \\
\hline $\min$ & minute(s) \\
\hline \multicolumn{2}{|l|}{ MSC } \\
\hline MTBF & mean time between failures \\
\hline MW & megawatt(s) \\
\hline MWR & morale, welfare and recreation \\
\hline NEC & National Electrical Code \\
\hline NEMA & National Electrical Manufacturers Association \\
\hline NSN & National Stock Numbers \\
\hline $\mathrm{PC}$ & personal computer \\
\hline PDF & portable document format \\
\hline PDU & power distribution unit \\
\hline PM FSS & U.S. Army Product Manager, Force Sustainment Systems \\
\hline PNNL & Pacific Northwest National Laboratory \\
\hline PV & photovoltaic \\
\hline $\mathrm{RS}$ & Recommended Standard \\
\hline RTU & remote terminal unit \\
\hline
\end{tabular}


$\mathrm{S}$

SAGE

SCADA

SEER

SF

SHW

SIP

SOC

SRCC

TCMS

TCP

TIAX

TMY3

TOGS

TQG

UFC

UFGS

UL

UMCS

USACE

USB

$\mathrm{V}$

VAC

W

$\mathrm{yr}$

"

second

Smart and Green Energy

Supervisory Control and Data Acquisition

Seasonal Energy Efficiency Ratio

solar fraction

solar hot water

structural insulated panel

state of charge

Solar Rating Certification Corporation

Theater Construction Management System

Transmission Control Protocol

Refers to an electrical equipment evaluation furnished by the Army and prepared by the company TIAX, LLC ${ }^{33}$

typical meteorological year data, third version

Theater Oriented Guide Specifications

Tactical Quiet Generator

Unified Facilities Criteria

Unified Facilities Guide Specifications

Underwriters Laboratories

Utility Monitoring and Control System

U.S. Army Corps of Engineers

Universal Serial Bus

volt

volts alternating current

watt

year

inch, inches

${ }^{33}$ TIAX. 2006. Force Provider Electrical Survey, Analysis, and Market Investigation - Final Report. TIAX Ref. No. D0324, TIAX, LLC, Lexington, Massachusetts. 


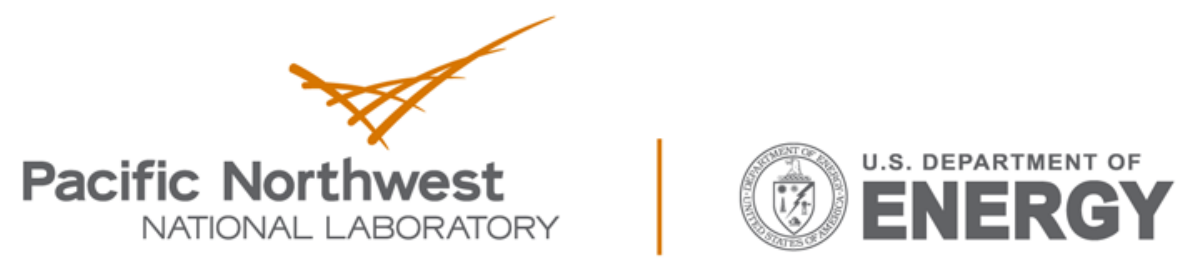

Proudly Operated by Battelle Since 1965

902 Battelle Boulevard

P.O. Box 999

Richland, WA 99352

1-888-375-PNNL (7665)

www.pnnl.gov 